\title{
Nucleophilic Substitution at Quaternary Carbon Stereocenters
}

Veeranjaneyulu Lanke and Ilan Marek*

Schulich Faculty of Chemistry. Technion - Israel Institute of Technology, Technion City 3200009, Haifa, Israel. 


\section{Table of Contents}

General Information

General Procedure for Starting Materials

SI-4

Characterization Data of the Newly Synthesized Starting Materials

SI-5

General Procedure for Nucleophilic Substitution Reaction

SI-9

Optimization Studies

SI-10

Characterization data of the new Compounds

SI-11

X-ray Crystal Structure of $\mathbf{2 m}$ and $\mathbf{2 s}$

SI-19

NMR Yields and Diastereomeric ratios calculation

SI-20

HPLC data for $\mathbf{2 q}$ and $\mathbf{5 b}$

SI-21

NMR Spectra

SI-22 


\section{General information}

All glassware was flame-dried under vacuum, and cooled under argon prior to use. Unless otherwise stated, all reactions were carried out under positive pressure of argon. Ether and THF were dried from Pure-Solv ${ }^{\circledR}$ Purification System (Innovative Technology $($ )). The newly opened commercial grade dichloromethane was used directly. Copper iodide, copper cyanide, copper bromide dimethyl sulfide, rhodium acetate dimer, methyllithium (1.6 $\mathrm{M}$ in diethyl ether), butyllithium (1.6 $\mathrm{M}$ in hexane), tertbutyllithium (1.7 M in pentane) were purchased from Aldrich. Methylmagnesium bromide (3.0 $\mathrm{M}$ in diethyl ether) was purchased from Acros. Ethylmagnesium bromide (2.0 $\mathrm{M}$ in diethyl ether), was prepared according to literature and freshly titrated before using with butanol/1,10-phenanthroline. Anhydrous $\mathrm{CuBr}_{2}$, purchased from Sigma Aldrich and used directly. Thin Layer Chromatography (TLC) was performed using Merck $\odot$ silica gel 60 F254 plates. Column chromatography was performed using Bio-Labsilica gel 60A $(0.040-0.063 \mathrm{~mm}) .{ }^{1} \mathrm{H}-\mathrm{NMR}$ and ${ }^{13} \mathrm{C}-\mathrm{NMR}$ spectra were recorded on a Bruker@spectrometers AVIII400 or Bruker Avance $300 \mathrm{NMR}$ using $\mathrm{CDCl}_{3}$ (unless otherwise specified) as solvent. The GC chromatograms were recorded using Varian (C) 3800 apparatus with Varian(C CP-Sil $8 \mathrm{CB}{ }^{\circledR}$ column. HPLC chromatograms were recorded using Agilent $(C) 1100$ Series line with CHIRALPAK ${ }^{\circledR}$ OX-H or CHIRALCEL® AY-H. 


\section{General Procedure for Starting Materials ${ }^{1}$}

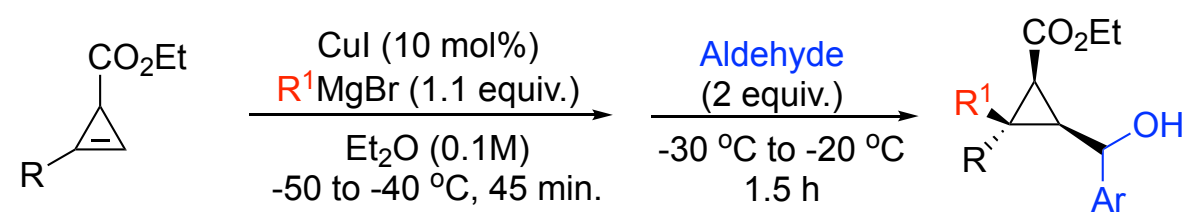

All the starting materials (1a to $\mathbf{1 p}$ ) were prepared according to the previously developed protocols in our group ${ }^{1}$ by modifying the nature of the electrophiles (to aldehydes).

Cyclopropene (1 equiv.) was added to a suspension of $\mathrm{CuI}(10 \mathrm{~mol} \%)$ in $\mathrm{Et}_{2} \mathrm{O}(0.1 \mathrm{M})$ and alkyl magnesium bromide ( 1.1 equiv.) was added dropwise at $-50{ }^{\circ} \mathrm{C}$. The resulting mixture (yellowish) was then stirred at -50 to $-35{ }^{\circ} \mathrm{C}$ for 45 minutes. Then, aldehyde ( 1.5 equiv.) was added and the reaction mixture was warmed to -35 to $-20^{\circ} \mathrm{C}$ during a period of $1.5 \mathrm{~h}$. An aqueous saturated solution of $\mathrm{NH}_{4} \mathrm{Cl}$ was then added. The aqueous layer was extracted twice with $\mathrm{Et}_{2} \mathrm{O}$. The combined organic phases were washed with brine, dried over $\mathrm{NaSO}_{4}$, filtered, and concentrated under reduced pressure. The crude mixture was purified by flash chromatography on silica gel by using a hexanes/ $\mathrm{Et}_{2} \mathrm{O}$ mixture to get pure products (the diastereomeric ratio on the three stereocenters on the cyclopropyl ring is $>$ 95:5:0:0 but the carbinol center is formed as two diastereomers in a 1:1 ratio).

Note: In most of the cases, the diastereomers were separated. In few cases, one of the diastereomer comes along with benzyl alcohol (addition of unreacted Grignard reagent with the aldehyde).

Scale of the reaction: It can be performed from $1 \mathrm{mmol}$ to $50 \mathrm{mmol}$.

\section{General procedure for the Synthesis of $1 q$ to $1 t^{1,2}$}<smiles>[R]C1CC1C(=O)OCC</smiles>

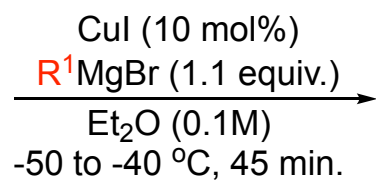

then $\mathrm{R}-\mathrm{X}$<smiles>[R]C1C(=O)C1[R]</smiles><smiles>[R20][GeH2]OC</smiles>

$\mathrm{R}^{2} \mathrm{MgBr}$ (1.1 equiv.)

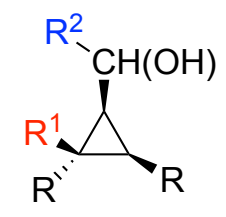

The starting materials (1q to $\mathbf{1 t}$ ) were prepared according to the previously developed protocols in our group ${ }^{2}$ by modifying the nature of the electrophile.

\section{Reduction of the Ester}

In a dry round-bottom flask containing the cyclopropyl ester $(10 \mathrm{mmol})$ in $20 \mathrm{~mL}$ of THF was added dropwise a solution of DIBAL-H (12 mmol) in hexane (1 M soln.), over a 15 minutes period at $0{ }^{\circ} \mathrm{C}$. When the addition was over, the reaction mixture was stirred for another 30 minutes at room temperature. After completion of the reaction (as monitored by TLC analysis), the reaction mixture was quenched with a saturated aqueous solution of $\mathrm{NH}_{4} \mathrm{Cl}$. The precipitate formed in the flask was dissolved by addition of a solution of $10 \mathrm{~mol} \% \mathrm{HCl}$ or Rochelle salt and the reaction mixture was extracted with $\mathrm{Et}_{2} \mathrm{O}(3 \times 25 \mathrm{~mL})$, dried over $\mathrm{MgSO}_{4}$, filtered and concentrated under vacuum to give the crude alcohol product which was further purified by column chromatography on silica gel using $20-30 \%$ diethyl ether in hexane as eluent. 


\section{Oxidation of Alcohol}

In a round-bottom flask, a solution of cyclopropyl alcohol $(5 \mathrm{mmol})$ and bis(acetoxy)iodobenzene (BAIB) (1.5 equiv) in DCM (20 mL), was added at $\mathrm{rt}$ (2,2,6,6-tetramethyl-1-piperidin-1-yl)oxy (TEMPO) (10 mol\%). The resulting reaction mixture was stirred for 2 to $4 \mathrm{~h}$. After completion (as monitored by TLC analysis), the reaction mixture was diluted with DCM $(20 \mathrm{~mL})$ and washed with a saturated aqueous solution of $\mathrm{Na}_{2} \mathrm{~S}_{2} \mathrm{O}_{3}(20 \mathrm{~mL})$. The aqueous layer was extracted with DCM $(2 \times 20$ $\mathrm{mL}$ ) and the combined organic layers were dried over $\mathrm{MgSO}_{4}$, filtered and evaporated under vacuum to give the crude aldehyde product which was further purified by column chromatography on silica gel using $5-10 \% \mathrm{Et}_{2} \mathrm{O}$ in hexane as eluent.

\section{Characterization data for 1a to 1 t.}

Ethyl (1S, 2S, 3R)-2-butyl-3-((S)-hydroxy(phenyl)methyl)-2-methylcyclopropane-1-carboxylate (1a): $\mathbf{R}_{\mathbf{f}}=0.5$ (ether/hexane $\left.=20: 80\right)$, colourless syrupy oil, 78\% yield, $1: 1 \mathrm{dr}$ determined by ${ }^{1} \mathrm{H}$ NMR. ${ }^{1} \mathrm{H}$ NMR (400 MHz, $\left.\mathrm{CDCl}_{3}\right) \delta 7.34-7.29(\mathrm{~m}, 4 \mathrm{H}), 7.28-7.25(\mathrm{~m}, 4 \mathrm{H}), 7.23-7.19(\mathrm{~m}, 1 \mathrm{H}), 7.18-7.17$ $(\mathrm{m}, 1 \mathrm{H}), 5.15(\mathrm{~d}, J=9.1 \mathrm{~Hz}, 1 \mathrm{H}), 5.02(\mathrm{~d}, J=10.1 \mathrm{~Hz}, 1 \mathrm{H}),, 4.09$ (q, $J=7.1 \mathrm{~Hz}, 2 \mathrm{H}), 3.96$ (ddd, $J=$ 9.8, 6.8, $3.2 \mathrm{~Hz}, 2 \mathrm{H}), 1.57(\mathrm{~d}, J=8.4 \mathrm{~Hz}, 1 \mathrm{H}), 1.45-1.38(\mathrm{~m}, 4 \mathrm{H}), 1.36(\mathrm{~s}, 3 \mathrm{H}), 1.20(\mathrm{dd}, J=11.9,4.8$ $\mathrm{Hz}, 14 \mathrm{H}), 1.09$ (t, $J=7.1 \mathrm{~Hz}, 6 \mathrm{H}), 0.82(\mathrm{t}, J=7.2 \mathrm{~Hz}, 3 \mathrm{H}), 0.71(\mathrm{t}, J=7.0 \mathrm{~Hz}, 3 \mathrm{H}) .{ }^{13} \mathrm{C}$ NMR $(101$ $\left.\mathrm{MHz}, \mathrm{CDCl}_{3}\right) \delta 172.8,171.7,144.4,144.0,128.4,128.4,127.4,127.3,126.2,125.9,125.4,69.3,69.0$, $60.6,60.1,42.6,42.1,39.8,39.7,30.6,30.2,29.4,29.3,28.6,28.2,22.8,22.5,14.4,14.3,14.1,14.0$, 12.8, 11.9. HRMS (APCI) calcd. for $\mathrm{C}_{18} \mathrm{H}_{25} \mathrm{O}_{2}\left[(\mathrm{M}+\mathrm{H})-\mathrm{H}_{2} \mathrm{O}\right] 273.1849$ found: 273.1853 .

Ethyl (1S, 2R, 3R)-2-butyl-3-((S)-hydroxy(phenyl)methyl)-2-methylcyclopropane-1-carboxylate (1b): $\mathrm{R}_{\mathrm{f}}=0.5$ (ether/hexane $=20: 80$ ), colourless syrupy oil, $72 \%$ yield, $1: 1 \mathrm{dr}$ determined by ${ }^{1} \mathrm{H}$ NMR (crude). Isolated both the diastereomers separately. ${ }^{1} \mathrm{H}$ NMR $\left(400 \mathrm{MHz}, \mathrm{CDCl}_{3}\right) \delta 7.34(\mathrm{~d}, J=7.5 \mathrm{~Hz}, 2 \mathrm{H}), 7.27$ (t, $J=7.5 \mathrm{~Hz}, 2 \mathrm{H}), 7.19$ (t, $J=7.2 \mathrm{~Hz}, 1 \mathrm{H}), 5.12$ (dd, $J=10.1,2.0 \mathrm{~Hz}, 1 \mathrm{H}$ ), 4.09 (dddd, $J=17.9,10.8$, $7.1,3.7 \mathrm{~Hz}, 2 \mathrm{H}), 2.78(\mathrm{~d}, J=3.1 \mathrm{~Hz}, 1 \mathrm{H}), 1.72(\mathrm{ddd}, J=13.8,11.3,4.9 \mathrm{~Hz}, 1 \mathrm{H}), 1.60(\mathrm{~d}, J=8.3 \mathrm{~Hz}$, $1 \mathrm{H}), 1.52-1.42(\mathrm{~m}, 2 \mathrm{H}), 1.30-1.14(\mathrm{~m}, 7 \mathrm{H}), 1.05(\mathrm{~s}, 3 \mathrm{H}), 0.79(\mathrm{t}, J=7.2 \mathrm{~Hz}, 3 \mathrm{H}) .{ }^{13} \mathrm{C}$ NMR $(101$ $\left.\mathrm{MHz}, \mathrm{CDCl}_{3}\right) \delta 172.9,144.2,128.4,127.3,126.1,77.4,77.1,76.8,68.9,60.6,41.1,30.8,29.5,28.9$, 28.6, 25.8, 23.0, 14.3, 14.1. HRMS (APCI) calcd. for $\mathrm{C}_{18} \mathrm{H}_{25} \mathrm{O}_{2}\left[(\mathrm{M}+\mathrm{H})-\mathrm{H}_{2} \mathrm{O}\right] 273.1849$ found: 273.1853 .

Ethyl (1S, 2S, 3R)-3-((S)-(4-bromophenyl)(hydroxy)methyl)-2-butyl-2-methylcyclopropane-1carboxylate $(\mathbf{1 c}): \mathrm{R}_{\mathrm{f}}=0.5$ (ether/hexane $=20: 80$ ), colourless syrupy oil, $74 \%$ yield, $1: 1 \mathrm{dr}$ determined by ${ }^{1} \mathrm{H}-\mathrm{NMR} .{ }^{1} \mathrm{H}$ NMR $\left(400 \mathrm{MHz}, \mathrm{CDCl}_{3}\right) \delta 7.39(\mathrm{dd}, J=8.4,2.1 \mathrm{~Hz}, 2 \mathrm{H}), 7.34(\mathrm{dd}, J=8.3,2.5 \mathrm{~Hz}$, $2 \mathrm{H}), 7.22-7.16(\mathrm{~m}, 4 \mathrm{H}), 5.13(\mathrm{dd}, J=9.7,2.8 \mathrm{~Hz}, 1 \mathrm{H}), 4.97(\mathrm{~d}, J=10.0 \mathrm{~Hz}, 1 \mathrm{H}), 4.13-4.04(\mathrm{~m}, 2 \mathrm{H})$, $4.01-3.93(\mathrm{~m}, 2 \mathrm{H}), 1.58(\mathrm{dd}, J=8.3,2.3 \mathrm{~Hz}, 1 \mathrm{H}), 1.45(\mathrm{dd}, J=8.9,2.2 \mathrm{~Hz}, 1 \mathrm{H}), 1.37-1.31(\mathrm{~m}, 6 \mathrm{H})$, 1.20 (ddd, $J=11.2,8.0,2.2 \mathrm{~Hz}, 13 \mathrm{H}), 1.14-1.08(\mathrm{~m}, 5 \mathrm{H}), 0.86-0.77(\mathrm{~m}, 5 \mathrm{H}), 0.76-0.70(\mathrm{~m}, 3 \mathrm{H})$. ${ }^{13} \mathrm{C}$ NMR $\left(101 \mathrm{MHz}, \mathrm{CDCl}_{3}\right) \delta 172.7,171.6,143.4,143.1,131.5,131.4,128.0,127.7,121.2,121.1$, $68.8,68.4,60.7,60.2,42.5,42.0,39.5,30.6,30.1,29.4,29.4,28.6,28.2,22.7,22.5,14.3,14.3,14.1$, 14.0, 12.9, 11.8. HRMS (APCI) calcd. for $\mathrm{C}_{18} \mathrm{H}_{24} \mathrm{BrO}_{2}\left[(\mathrm{M}+\mathrm{H})-\mathrm{H}_{2} \mathrm{O}\right] 351.0954$ found: 351.0964 .

Ethyl (1S, 2S, 3R)-3-((S)-benzo[d][1,3]dioxol-5-yl(hydroxy)methyl)-2-butyl-2-methylcyclopropane-1carboxylate (1d): $R_{\mathrm{f}}=0.5$ (ether/hexane $=20: 80$ ), colourless syrupy oil, $70 \%$ yield, $1: 0.8 \mathrm{dr}$ determined by ${ }^{1} \mathrm{H}-\mathrm{NMR} .{ }^{1} \mathrm{H}$ NMR $\left(400 \mathrm{MHz}, \mathrm{CDCl}_{3}\right) \delta 6.84(\mathrm{dd}, J=4.4,1.6 \mathrm{~Hz}, 2 \mathrm{H}), 6.76(\mathrm{~d}, J=1.6 \mathrm{~Hz}, 1 \mathrm{H})$, $6.74(\mathrm{~d}, J=1.6 \mathrm{~Hz}, 1 \mathrm{H}), 6.68(\mathrm{~d}, J=8.0 \mathrm{~Hz}, 1 \mathrm{H}), 6.63(\mathrm{~d}, J=8.0 \mathrm{~Hz}, 1 \mathrm{H}), 5.85(\mathrm{~s}, 2 \mathrm{H}), 5.82(\mathrm{~s}, 2 \mathrm{H})$, $5.05(\mathrm{~d}, J=9.7 \mathrm{~Hz}, 1 \mathrm{H}), 4.93(\mathrm{~d}, J=10.1 \mathrm{~Hz}, 1 \mathrm{H}), 4.08(\mathrm{q}, J=7.1 \mathrm{~Hz}, 2 \mathrm{H}), 4.02-3.91(\mathrm{~m}, 2 \mathrm{H}), 1.56$ $(\mathrm{d}, J=8.4 \mathrm{~Hz}, 1 \mathrm{H}), 1.43(\mathrm{~d}, J=9.0 \mathrm{~Hz}, 1 \mathrm{H}), 1.39-1.32(\mathrm{~m}, 6 \mathrm{H}), 1.25-1.15(\mathrm{~m}, 12 \mathrm{H}), 1.10(\mathrm{td}, J=$ 7.0, $2.9 \mathrm{~Hz}, 6 \mathrm{H}), 0.82(\mathrm{t}, J=7.2 \mathrm{~Hz}, 4 \mathrm{H}), 0.73(\mathrm{t}, J=7.0 \mathrm{~Hz}, 3 \mathrm{H}) .{ }^{13} \mathrm{C} \mathrm{NMR}\left(101 \mathrm{MHz}, \mathrm{CDCl}_{3}\right) \delta 172.6$, 171.6, 147.7, 147.6, 146.7, 146.7, 138.5, 138.2, 119.5, 119.2, 108.0, 107.9, 106.8, 106.6, 100.9, 100.87, $69.1,68.7,60.5,60.1,42.5,42.1,39.7,30.6,30.0,29.3,29.1,28.6,28.2,22.7,22.5,14.3,14.3,14.1$, 134.0, 12.7, 11.8. HRMS (APCI) calcd. for $\mathrm{C}_{19} \mathrm{H}_{25} \mathrm{O}_{4}\left[(\mathrm{M}+\mathrm{H})-\mathrm{H}_{2} \mathrm{O}\right] 317.1747$ found: 317.1764 . 
Ethyl (1S, 2S, 3R)-2-butyl-3-((S)-hydroxy(naphthalen-1-yl)methyl)-2-methylcyclopropane-1carboxylate $(1 \mathrm{e}): \mathrm{R}_{\mathrm{f}}=0.5$ (ether/hexane $=20: 80$ ), colourless syrupy oil, $76 \%$ yield, $1: 1 \mathrm{dr}$ by crude NMR, but isolated as one of the major isomers (44\% Yield). ${ }^{1} \mathrm{H}$ NMR $\left(400 \mathrm{MHz}, \mathrm{CDCl}_{3}\right) \delta 8.46(\mathrm{~d}, J$ $=8.4 \mathrm{~Hz}, 1 \mathrm{H}), 7.91-7.84(\mathrm{~m}, 1 \mathrm{H}), 7.81(\mathrm{~d}, J=8.2 \mathrm{~Hz}, 1 \mathrm{H}), 7.58(\mathrm{~d}, J=6.4 \mathrm{~Hz}, 1 \mathrm{H}), 7.55-7.43(\mathrm{~m}$, $3 \mathrm{H}), 5.82(\mathrm{dd}, J=10.0,3.1 \mathrm{~Hz}, 1 \mathrm{H}), 4.24(\mathrm{q}, J=7.1 \mathrm{~Hz}, 2 \mathrm{H}), 2.87(\mathrm{~d}, J=3.7 \mathrm{~Hz}, 1 \mathrm{H}), 1.97(\mathrm{dd}, J=$ 10.0, 8.4 Hz, 1H), $1.77(\mathrm{~d}, J=8.4 \mathrm{~Hz}, 1 \mathrm{H}), 1.71-1.61(\mathrm{~m}, 1 \mathrm{H}), 1.34(\mathrm{t}, J=7.1 \mathrm{~Hz}, 3 \mathrm{H}), 1.30-1.23$ $(\mathrm{m}, 2 \mathrm{H}), 1.18(\mathrm{~s}, 3 \mathrm{H}), 1.13-1.05(\mathrm{~m}, 2 \mathrm{H}), 0.74(\mathrm{t}, J=7.1 \mathrm{~Hz}, 3 \mathrm{H}) \cdot{ }^{13} \mathrm{C}$ NMR $\left(101 \mathrm{MHz}, \mathrm{CDCl}_{3}\right) \delta$ $172.9,138.9,134.3,131.2,128.8,128.5,128.3,126.0,125.6,125.3,124.7,124.2,67.7,60.7,42.1,38.3$, $30.4,29.2,28.2,22.4,14.4,13.9,12.4$. HRMS (APCI) calcd. for $\mathrm{C}_{22} \mathrm{H}_{27} \mathrm{O}_{2}\left[(\mathrm{M}+\mathrm{H})-\mathrm{H}_{2} \mathrm{O}\right] 323.2006$ found: 323.2002 .

Ethyl (1S, 2S, 3R)-2-butyl-3-((S)-(2,4-difluorophenyl)(hydroxy)methyl)-2-methylcyclopropane-1carboxylate (1f): $\mathrm{R}_{\mathrm{f}}=0.5$ (ether/hexane $=20: 80$ ), colourless syrupy oil, [Crude-65\% yield, $1: 1 \mathrm{dr}$ ]. Isolated as a one of the single isomers, $42 \%$ yield. ${ }^{1} \mathrm{H}$ NMR $\left(400 \mathrm{MHz}, \mathrm{CDCl}_{3}\right) \delta 7.44(\mathrm{dt}, J=17.4,9.6$ $\mathrm{Hz}, 1 \mathrm{H}), 6.82(\mathrm{t}, J=8.4 \mathrm{~Hz}, 1 \mathrm{H}), 6.75-6.67(\mathrm{~m}, 1 \mathrm{H}), 5.26(\mathrm{dd}, J=10.0,3.5 \mathrm{~Hz}, 1 \mathrm{H}), 4.11(\mathrm{q}, J=7.1$ $\mathrm{Hz}, 2 \mathrm{H}), 3.02(\mathrm{~d}, J=3.3 \mathrm{~Hz}, 1 \mathrm{H}), 1.59(\mathrm{~d}, J=8.3 \mathrm{~Hz}, 1 \mathrm{H}), 1.46-1.39(\mathrm{~m}, 2 \mathrm{H}), 1.22(\mathrm{t}, J=7.1 \mathrm{~Hz}$, $5 \mathrm{H}), 1.14(\mathrm{~d}, J=1.1 \mathrm{~Hz}, 3 \mathrm{H}), 1.09-1.04(\mathrm{~m}, 3 \mathrm{H}), 0.83(\mathrm{t}, J=7.2 \mathrm{~Hz}, 1 \mathrm{H}), 0.72(\mathrm{t}, J=7.0 \mathrm{~Hz}, 3 \mathrm{H}) .{ }^{13} \mathrm{C}$ NMR $\left(101 \mathrm{MHz}, \mathrm{CDCl}_{3}\right) \delta \delta 172.0(\mathrm{~d}, J=162.7 \mathrm{~Hz}), 161.0(\mathrm{~d}, J=12.0 \mathrm{~Hz}), 128.9(\mathrm{dd}, J=9.7,6.4$ $\mathrm{Hz}), 111.5(\mathrm{dd}, J=21.0,3.6 \mathrm{~Hz}), 103.7(\mathrm{t}, J=25.7 \mathrm{~Hz}), 63.9(\mathrm{~d}, J=2.7 \mathrm{~Hz}), 60.5(\mathrm{~d}, J=58.6 \mathrm{~Hz})$, 42.1, 38.8, 30.4, 29.6, 28.2, 22.4, 14.4, 13.9, 12.3 (d, $J=3.5 \mathrm{~Hz}$ ). HRMS (APCI) calcd. for $\mathrm{C}_{18} \mathrm{H}_{23} \mathrm{~F}_{2} \mathrm{O}_{2}$ $\left[(\mathrm{M}+\mathrm{H})-\mathrm{H}_{2} \mathrm{O}\right] 309.1661$ found: 309.1677 .

Ethyl (1S, 2S, 3R)-2-butyl-3-((S)-furan-2-yl(hydroxy)methyl)-2-methylcyclopropane-1-carboxylate $(\mathbf{1 g}): R_{\mathrm{f}}=0.4$ (ether/hexane $\left.=20: 80\right)$, colourless syrupy oil, 46\% yield, 1:1 dr. ${ }^{1} \mathrm{H}$ NMR $(400 \mathrm{MHz}$, $\left.\mathrm{CDCl}_{3}\right) \delta 7.31(\mathrm{~d}, J=14.1 \mathrm{~Hz}, 2 \mathrm{H}), 6.25(\mathrm{~d}, J=17.8 \mathrm{~Hz}, 2 \mathrm{H}), 6.16(\mathrm{dd}, J=19.4,2.9 \mathrm{~Hz}, 2 \mathrm{H}), 5.18(\mathrm{dd}$, $J=9.5,4.6 \mathrm{~Hz}, 1 \mathrm{H}), 5.10(\mathrm{dd}, J=9.8,4.2 \mathrm{~Hz}, 1 \mathrm{H}), 4.10(\mathrm{q}, J=7.1 \mathrm{~Hz}, 2 \mathrm{H}), 3.96(\mathrm{p}, J=6.9 \mathrm{~Hz}, 2 \mathrm{H})$, $2.57(\mathrm{~d}, J=4.0 \mathrm{~Hz}, 1 \mathrm{H}), 2.16(\mathrm{dt}, J=21.5,9.1 \mathrm{~Hz}, 2 \mathrm{H}), 1.91(\mathrm{~d}, J=4.9 \mathrm{~Hz}, 1 \mathrm{H}), 1.59$ (dd, $J=6.6,3.7$ $\mathrm{Hz}, 1 \mathrm{H}), 1.54(\mathrm{~s}, 3 \mathrm{H}), 1.33(\mathrm{~s}, 3 \mathrm{H}), 1.23(\mathrm{dd}, J=13.3,6.3 \mathrm{~Hz}, 10 \mathrm{H}), 1.10(\mathrm{t}, J=7.1 \mathrm{~Hz}, 6 \mathrm{H}), 0.81(\mathrm{dt}$, $J=20.8,7.0 \mathrm{~Hz}, 6 \mathrm{H}) .{ }^{13} \mathrm{C}$ NMR $\left(101 \mathrm{MHz}, \mathrm{CDCl}_{3}\right) \delta 172.4,171.1,156.0,142.1,142.1,110.1,105.9$, 63.7, 63.4, 60.6, 60.1, 42.4, 42.1, 36.2, 35.7, 29.91, 29.86, 29.3, 28.8, 28.7, 28.2, 22.8, 22.558, 14.4, 14.3, 14.12, 14.07 12.2, 11.6. HRMS (APCI) calcd. for $\mathrm{C}_{16} \mathrm{H}_{23} \mathrm{O}_{3}\left[(\mathrm{M}+\mathrm{H})-\mathrm{H}_{2} \mathrm{O}\right] 263.1642$ found: 263.1651 .

Ethyl (1S, 2S, 3R)-2-butyl-3-((S)-1-hydroxy-3-phenylprop-2-yn-1-yl)-2-methylcyclopropane-1carboxylate $(\mathbf{1 h}): \mathrm{R}_{\mathrm{f}}=0.6$ (ether/hexane $=20: 80$ ), colourless syrupy oil, $62 \%$ yield, $1: 1 \mathrm{dr} .{ }^{1} \mathrm{H}$ NMR $\left(400 \mathrm{MHz}, \mathrm{CDCl}_{3}\right) \delta 7.32(\mathrm{dd}, J=6.6,3.0 \mathrm{~Hz}, 2 \mathrm{H}), 7.19(\mathrm{dd}, J=5.0,1.6 \mathrm{~Hz}, 3 \mathrm{H}), 5.02(\mathrm{~d}, J=9.6 \mathrm{~Hz}$, 1H), 4.04 (dddd, $J=17.8,10.8,7.1,3.7 \mathrm{~Hz}, 2 \mathrm{H}), 1.59(\mathrm{~d}, J=9.0 \mathrm{~Hz}, 1 \mathrm{H}), 1.52(\mathrm{t}, J=9.3 \mathrm{~Hz}, 1 \mathrm{H}), 1.32$ $(\mathrm{dd}, J=12.4,6.0 \mathrm{~Hz}, 2 \mathrm{H}), 1.26(\mathrm{~s}, 3 \mathrm{H}), 1.24-1.18(\mathrm{~m}, 3 \mathrm{H}), 1.14(\mathrm{~d}, J=7.1 \mathrm{~Hz}, 4 \mathrm{H}), 0.82(\mathrm{t}, J=7.2$ $\mathrm{Hz}, 3 \mathrm{H}) .{ }^{13} \mathrm{C}$ NMR $\left(101 \mathrm{MHz}, \mathrm{CDCl}_{3}\right) \delta 172.2,171.1,131.8,131.7,128.3,128.2,60.6,60.3,59.0,42.2$, 42.2, 38.3, 38.1, 30.5, 29.3, 28.6, 22.7, 14.4, 14.2, 14.1, 12.1, 11.5. HRMS (APCI) calcd. for $\mathrm{C}_{20} \mathrm{H}_{25} \mathrm{O}_{2}$ [(M+H)- $\left.\mathrm{H}_{2} \mathrm{O}\right] 297.1849$ found: 297.1830 .

Ethyl (1S, 2S, 3R)-2-butyl-3-((S)-1-hydroxy-3-(triisopropylsilyl)prop-2-yn-1-yl)-2 methylcyclopropane-1-carboxylate $(\mathbf{1 i}): \mathrm{R}_{\mathrm{f}}=0.5$ (ether/hexane $=20: 80$ ), colourless syrupy oil, $48 \%$ yield, single diastereomer. ${ }^{1} \mathrm{H}$ NMR $\left(400 \mathrm{MHz}, \mathrm{CDCl}_{3}\right) \delta 4.81(\mathrm{dd}, J=9.8,4.6 \mathrm{~Hz}, 1 \mathrm{H}), 4.06(\mathrm{q}, J=$ $7.1 \mathrm{~Hz}, 2 \mathrm{H}), 2.21(\mathrm{~d}, J=4.6 \mathrm{~Hz}, 1 \mathrm{H}), 1.55-1.48(\mathrm{~m}, 2 \mathrm{H}), 1.37-1.29(\mathrm{~m}, 3 \mathrm{H}), 1.20(\mathrm{t}, J=7.1 \mathrm{~Hz}, 5 \mathrm{H})$, $1.16(\mathrm{~s}, 3 \mathrm{H}), 1.03-0.94(\mathrm{~m}, 21 \mathrm{H}), 0.81(\mathrm{t}, J=7.2 \mathrm{~Hz}, 3 \mathrm{H}) .{ }^{13} \mathrm{C} \mathrm{NMR}\left(101 \mathrm{MHz}, \mathrm{CDCl}_{3}\right) \delta 172.0,107.8$, 84.6, 60.5, 58.9, 42.3, 38.2, 29.7, 29.0, 28.6, 22.8, 18.6, 14.3, 14.1, 12.0, 11.2. HRMS (APCI) calcd. for $\mathrm{C}_{23} \mathrm{H}_{41} \mathrm{O}_{2} \mathrm{Si}\left[(\mathrm{M}+\mathrm{H})-\mathrm{H}_{2} \mathrm{O}\right] 377.2870$ found: 377.2896 .

Ethyl (1S, 2S, 3R)-2-hexyl-3-((S)-hydroxy(phenyl)methyl)-2-methylcyclopropane-1-carboxylate (1j): $\mathrm{R}_{\mathrm{f}}=0.6$ (ether/hexane $=20: 80$ ), colourless syrupy oil, $72 \%$ yield, $1: 1 \mathrm{dr} .{ }^{1} \mathrm{H}$ NMR $\left(400 \mathrm{MHz}, \mathrm{CDCl}_{3}\right)$ $\delta 7.37-7.31(\mathrm{~m}, 2 \mathrm{H}), 7.29-7.23(\mathrm{~m}, 2 \mathrm{H}), 7.21-7.18(\mathrm{~m}, 1 \mathrm{H}), 5.20(\mathrm{dd}, J=9.3,3.4 \mathrm{~Hz}, 1 \mathrm{H}), 4.04-$ $3.95(\mathrm{~m}, 2 \mathrm{H}), 1.79(\mathrm{~d}, J=3.5 \mathrm{~Hz}, 1 \mathrm{H}), 1.47(\mathrm{~d}, J=9.0 \mathrm{~Hz}, 1 \mathrm{H}), 1.38(\mathrm{~s}, 3 \mathrm{H}), 1.24-1.18(\mathrm{~m}, 7 \mathrm{H}), 1.11$ $(\mathrm{t}, J=7.1 \mathrm{~Hz}, 3 \mathrm{H}), 0.85-0.78(\mathrm{~m}, 4 \mathrm{H}) .{ }^{13} \mathrm{C}$ NMR $\left(101 \mathrm{MHz}, \mathrm{CDCl}_{3}\right) \delta 171.7,144.3,128.4,127.5$, 126.2, 69.1, 60.1, 42.8, 39.7, 31.9, 30.6, 29.5, 29.3, 26.5, 22.7, 14.3, 14.12 11.9. HRMS (APCI) calcd. for $\mathrm{C}_{20} \mathrm{H}_{27} \mathrm{O}_{3}\left[(\mathrm{M}+\mathrm{H})-\mathrm{H}_{2} \mathrm{O}\right] 315.1955$ found: 315.1969 . 
Ethyl (1S, 2S, 3R)-2-(2-(benzyloxy)ethyl)-3-((S)-hydroxy(phenyl)methyl)-2-methylcyclopropane-1carboxylate $(\mathbf{1 k}): \mathrm{R}_{\mathrm{f}}=0.4$ (ether/hexane $=35: 65$ ), colourless syrupy oil, 64\% yield, 1:0.9 dr. ${ }^{1} \mathrm{H}$ NMR $\left(400 \mathrm{MHz}, \mathrm{CDCl}_{3}\right) \delta 7.33(\mathrm{~s}, 2 \mathrm{H}), 7.31-7.15(\mathrm{~m}, 18 \mathrm{H}), 5.14(\mathrm{~d}, J=9.0 \mathrm{~Hz}, 1 \mathrm{H}), 5.04(\mathrm{~d}, J=10.1 \mathrm{~Hz}$, $1 \mathrm{H}), 4.47(\mathrm{~s}, 2 \mathrm{H}), 4.25(\mathrm{~s}, 2 \mathrm{H}), 4.12(\mathrm{tt}, J=7.1,3.5 \mathrm{~Hz}, 2 \mathrm{H}), 4.04-3.96(\mathrm{~m}, 2 \mathrm{H}), 3.68(\mathrm{td}, J=9.2,5.3$ $\mathrm{Hz}, 1 \mathrm{H}), 3.57-3.50(\mathrm{~m}, 1 \mathrm{H}), 3.40(\mathrm{q}, J=7.0 \mathrm{~Hz}, 4 \mathrm{H}), 3.35-3.22(\mathrm{~m}, 2 \mathrm{H}), 1.87-1.77(\mathrm{~m}, 1 \mathrm{H}), 1.72$ $(\mathrm{d}, J=8.5 \mathrm{~Hz}, 1 \mathrm{H}), 1.63(\mathrm{dd}, J=14.0,7.1 \mathrm{~Hz}, 1 \mathrm{H}), 1.54-1.46(\mathrm{~m}, 1 \mathrm{H}), 1.40(\mathrm{~s}, 3 \mathrm{H}), 1.24(\mathrm{~s}, 3 \mathrm{H}), 1.13$ $(\operatorname{td}, J=7.1,4.4 \mathrm{~Hz}, 6 \mathrm{H}) .{ }^{13} \mathrm{C} \mathrm{NMR}\left(101 \mathrm{MHz}, \mathrm{CDCl}_{3}\right) \delta 172.5,171.4,144.4,143.9,138.3,137.9,128.53$, $128.50,128.436,128.3,128.0,127.9,127.6,127.5,127.4,127.3,126.1,125.9,73.4,73.0,69.4,70.0$, 68.0, 67.7, 65.9, 60.7, 60.2, 41.8, 41.7, 40.0, 39.0, 29.4, 29.1, 28.1, 27.4, 15.3, 14.4, 14.3, 13.5, 11.5 . HRMS (APCI) calcd. for $\mathrm{C}_{23} \mathrm{H}_{27} \mathrm{O}_{3}\left[(\mathrm{M}+\mathrm{H})-\mathrm{H}_{2} \mathrm{O}\right] 351.1955$ found: 351.1980 .

Ethyl (1S, 2S, 3R)-2-butyl-3-((R)-1-hydroxyallyl)-2-methylcyclopropane-1-carboxylate (11): $\mathrm{R}_{\mathrm{f}}=0.5$ (ether/hexane $=15: 85)$, colourless syrupy oil, 65\% yield, 1:0.75 dr. ${ }^{1} \mathrm{H}$ NMR $(400 \mathrm{MHz}, \mathrm{CDCl} 3) \delta 5.85$ (dddd, $\mathrm{J}=16.2,12.8,10.5,5.5 \mathrm{~Hz}, 2 \mathrm{H}), 5.28-5.13(\mathrm{~m}, 2 \mathrm{H}), 5.05(\mathrm{~d}, \mathrm{~J}=10.5 \mathrm{~Hz}, 1 \mathrm{H}), 4.99(\mathrm{~d}, \mathrm{~J}=$ $10.5 \mathrm{~Hz}, 1 \mathrm{H}), 4.57(\mathrm{dd}, \mathrm{J}=9.6,5.5 \mathrm{~Hz}, 1 \mathrm{H}), 4.48(\mathrm{dd}, \mathrm{J}=9.7,5.6 \mathrm{~Hz}, 1 \mathrm{H}), 4.15-3.92(\mathrm{~m}, 4 \mathrm{H}), 2.47$ $(\mathrm{s}, 1 \mathrm{H}), 1.84(\mathrm{~s}, 1 \mathrm{H}), 1.50(\mathrm{t}, \mathrm{J}=8.5 \mathrm{~Hz}, 2 \mathrm{H}), 1.36-1.09(\mathrm{~m}, 22 \mathrm{H}), 0.88-0.77(\mathrm{~m}, 6 \mathrm{H}) .{ }^{13} \mathrm{C}$ NMR $(101$ $\mathrm{MHz}, \mathrm{CDCl} 3) \delta 172.4,171.4,140.3,140.1,114.3,114.0,68.3,67.9,60.4,60.1,42.4,42.2,37.5,37.4$, 29.7, 29.6, 29.0, 28.7, 28.6, 28.4, 22.7, 22.7, 14.4, 14.3, 14.1, 14.0, 12.3, 11.7. HRMS (APCI) calcd. for $\mathrm{C}_{14} \mathrm{H}_{23} \mathrm{O}_{2}\left[(\mathrm{M}+\mathrm{H})-\mathrm{H}_{2} \mathrm{O}\right] 223.1693$ found: 223.1690 .

Ethyl (1S, 2S, 3R)-2-butyl-3-((R)-(4-((S)-((1S,2S,3S)-2-butyl-3-(ethoxycarbonyl)-2methylcyclopropyl)(hydroxy)methyl)phenyl)(hydroxy)methyl)-2-methylcyclopropane-1-carboxylate $(\mathbf{1 m}): \mathrm{R}_{\mathrm{f}}=0.5(\mathrm{ether} / \mathrm{hexane}=60: 40)$, colourless syrupy oil, 30\% yield, 1:2.5 dr. ${ }^{1} \mathrm{H}$ NMR $(400 \mathrm{MHz}$, $\left.\mathrm{CDCl}_{3}\right) \delta 7.36-7.26(\mathrm{~m}, 7 \mathrm{H}(2+5)), 5.18(\mathrm{~d}, J=8.6 \mathrm{~Hz}, 1 \mathrm{H}), 5.03(\mathrm{t}, J=9.9 \mathrm{~Hz}, 2.5 \mathrm{H}), 4.12(\mathrm{q}, J=7.1$ $\mathrm{Hz}, 5 \mathrm{H}), 4.04-3.90(\mathrm{~m}, 2 \mathrm{H}), 2.72(\mathrm{~s}, 2 \mathrm{H}), 1.60(\mathrm{~d}, J=8.3 \mathrm{~Hz}, 2 \mathrm{H}), 1.46-1.34(\mathrm{~m}, 10 \mathrm{H}), 1.25-1.16$ $(\mathrm{m}, 23 \mathrm{H}), 1.13-1.06(\mathrm{~m}, 10 \mathrm{H}), 0.86-0.78(\mathrm{~m}, 6 \mathrm{H}), 0.73(\mathrm{t}, J=6.7 \mathrm{~Hz}, 8 \mathrm{H}) .{ }^{13} \mathrm{C}$ NMR $(101 \mathrm{MHz}$, $\left.\mathrm{CDCl}_{3}\right) \delta 172.8,171.6,143.1,143.0,126.3,126.0,125.9,69.3,68.9,60.6,60.1,42.6,42.1,39.7,39.7$, $30.6,30.2,29.5,29.3,28.7,28.2,22.8,22.6,14.39,14.35,14.1,14.0,12.9,11.9$. HRMS (APCI) calcd. for $\mathrm{C}_{30} \mathrm{H}_{45} \mathrm{O}_{5}\left[(\mathrm{M}+\mathrm{H})-\mathrm{H}_{2} \mathrm{O}\right] 485.3262$ found: 485.3246 .

Benzyl (1S, 2S, 3R)-2-butyl-3-((R)-hydroxy(phenyl)methyl)-2-methylcyclopropane-1-carboxylate $(1 \mathrm{n}): \mathrm{R}_{\mathrm{f}}=0.2$ (ether/hexane $=20: 80$ ), colourless syrupy oil, $65 \%$ yield, 0.66:0.33 dr, isolated as a major single diastereomer. ${ }^{1} \mathrm{H}$ NMR $\left(400 \mathrm{MHz}, \mathrm{CDCl}_{3}\right) \delta 7.29-7.22(\mathrm{~m}, 5 \mathrm{H}), 7.22-7.15(\mathrm{~m}, 5 \mathrm{H}), 5.17(\mathrm{dd}$, $J=9.8,2.7 \mathrm{~Hz}, 1 \mathrm{H}), 5.04(\mathrm{~d}, J=12.4 \mathrm{~Hz}, 1 \mathrm{H}), 4.91(\mathrm{~d}, J=12.4 \mathrm{~Hz}, 1 \mathrm{H}), 1.81(\mathrm{~s}, 1 \mathrm{H}), 1.53(\mathrm{~d}, J=9.0$ $\mathrm{Hz}, 1 \mathrm{H}), 1.44(\mathrm{t}, J=9.4 \mathrm{~Hz}, 1 \mathrm{H}), 1.38(\mathrm{~s}, 3 \mathrm{H}), 1.37-1.31(\mathrm{~m}, 2 \mathrm{H}), 1.31-1.20(\mathrm{~m}, 4 \mathrm{H}), 0.82(\mathrm{t}, J=7.2$ $\mathrm{Hz}, 3 \mathrm{H}) .{ }^{13} \mathrm{C}$ NMR $\left(101 \mathrm{MHz}, \mathrm{CDCl}_{3}\right) \delta 171.5,144.2,136.3,128.5,128.5,128.2,128.1,127.5,126.2$, $69.0,70.0,42.5,40.0,31.1,29.4,28.7,22.8,14.1,12.0$. HRMS (APCI) calcd. for $\mathrm{C}_{23} \mathrm{H}_{27} \mathrm{O}_{2}[(\mathrm{M}+\mathrm{H})-$ $\left.\mathrm{H}_{2} \mathrm{O}\right] 335.2006$ found: 335.2010 .

(S)-((1R, 2R, 3S)-2-butyl-3-(hydroxymethyl)-2-methylcyclopropyl)(phenyl)methanol (10): $\mathrm{R}_{\mathrm{f}}=0.4$ (ether/hexane $=50: 50)$, colourless syrupy oil, $85 \%$ yield, single diastereomer. ${ }^{1} \mathrm{H}$ NMR $(400 \mathrm{MHz}$, $\left.\mathrm{CDCl}_{3}\right) \delta 7.34(\mathrm{~d}, J=7.2 \mathrm{~Hz}, 2 \mathrm{H}), 7.29(\mathrm{t}, J=7.4 \mathrm{~Hz}, 2 \mathrm{H}), 7.22(\mathrm{dd}, J=8.3,6.0 \mathrm{~Hz}, 1 \mathrm{H}), 4.41(\mathrm{~d}, J=$ $10.3 \mathrm{~Hz}, 1 \mathrm{H}), 3.58(\mathrm{~d}, J=6.2 \mathrm{~Hz}, 2 \mathrm{H}), 1.81(\mathrm{~s}, 1 \mathrm{H}), 1.63(\mathrm{~s}, 1 \mathrm{H}), 1.37-1.30(\mathrm{~m}, 2 \mathrm{H}), 1.27-1.18(\mathrm{~m}$, $7 \mathrm{H}), 1.13-1.06(\mathrm{~m}, 1 \mathrm{H}), 0.90(\mathrm{t}, J=7.4 \mathrm{~Hz}, 1 \mathrm{H}), 0.84(\mathrm{t}, J=7.1 \mathrm{~Hz}, 3 \mathrm{H}) \cdot{ }^{13} \mathrm{C}$ NMR $\left(101 \mathrm{MHz}, \mathrm{CDCl}_{3}\right)$ $\delta 144.3,128.8,127.9,126.2,77.4,77.1,76.8,71.7,60.1,42.6,34.9,29.5,29.0,23.6,22.9,14.2,12.5$. HRMS (APCI) calcd. for $\mathrm{C}_{16} \mathrm{H}_{23} \mathrm{O}_{2}\left[(\mathrm{M}+\mathrm{H})-\mathrm{H}_{2} \mathrm{O}\right] 247.1693$ found: 247.1704 .

Ethyl (1S,2R,3R)-2-butyl-3-((R)-hydroxy(phenyl)methyl)-2-phenylcyclopropane-1-carboxylate (1p): $\mathrm{Rf}=0.5$ (ether/hexane $=20: 80)$, colourless syrupy oil, $59 \%$ yield, $80: 20 \mathrm{dr} .{ }^{1} \mathrm{H} \mathrm{NMR}\left(400 \mathrm{MHz}, \mathrm{CDCl}_{3}\right)$ $\delta 7.51-7.04(\mathrm{~m}, 10 \mathrm{H}), 4.92(\mathrm{~d}, J=7.7 \mathrm{~Hz}, 1 \mathrm{H}), 3.94-3.69(\mathrm{~m}, 2 \mathrm{H}), 2.00(\mathrm{~s}, 1 \mathrm{H}), 1.83(\mathrm{~d}, J=7.5 \mathrm{~Hz}$, 2H), $1.66(\mathrm{t}, J=11.8 \mathrm{~Hz}, 1 \mathrm{H}), 1.36(\mathrm{~d}, J=13.0 \mathrm{~Hz}, 1 \mathrm{H}), 1.31-1.08(\mathrm{~m}, 4 \mathrm{H}), 0.91(\mathrm{t}, J=6.7 \mathrm{~Hz}, 3 \mathrm{H})$, 0.75 (s, 3H). ${ }^{13} \mathrm{C}$ NMR $\left(101 \mathrm{MHz}, \mathrm{CDCl}_{3}\right) \delta 170.3,144.2,137.5,130.8,128.4,128.1,127.7,126.9$, 126.6, 70.0, 60.1, 44.7, 41.2, 38.9, 30.1, 28.8, 22.6, 14.1, 14.1. HRMS (APCI) calcd. for $\mathrm{C}_{23} \mathrm{H}_{27} \mathrm{O}_{2}$ $\left[(\mathrm{M}+\mathrm{H})-\mathrm{H}_{2} \mathrm{O}\right] 335.2010$ found: 335.2014 .

(S)-((1R, 2R, 3S)-3-allyl-2-butyl-2-methylcyclopropyl)(4-methoxyphenyl)methanol (1q): $\mathrm{R}_{\mathrm{f}}=0.4$ $($ ether/hexane $=20: 80$ ), colourless syrupy oil, $70 \%$ yield (over two steps-Oxidation and reduction), 1:1 
dr. ${ }^{1} \mathrm{H}$ NMR $\left(400 \mathrm{MHz}, \mathrm{CDCl}_{3}\right) \delta 7.30-7.22(\mathrm{~m}, 4 \mathrm{H}), 6.81(\mathrm{dd}, J=8.7,1.5 \mathrm{~Hz}, 4 \mathrm{H}), 6.03-5.91(\mathrm{~m}$, $1 \mathrm{H}), 5.71-5.57(\mathrm{~m}, 1 \mathrm{H}), 5.18-5.09(\mathrm{~m}, 1 \mathrm{H}), 5.04-4.97(\mathrm{~m}, 1 \mathrm{H}), 4.90(\mathrm{dd}, J=17.1,1.8 \mathrm{~Hz}, 1 \mathrm{H})$, $4.83(\mathrm{dd}, J=10.2,1.8 \mathrm{~Hz}, 1 \mathrm{H}), 4.35(\mathrm{~d}, J=5.1 \mathrm{~Hz}, 1 \mathrm{H}), 4.33(\mathrm{~d}, J=5.1 \mathrm{~Hz}, 1 \mathrm{H}), 3.73(\mathrm{~s}, 6 \mathrm{H}), 2.27-$ 2.20 (m, 2H), 2.04 (ddd, $J=7.0,6.3,1.2 \mathrm{~Hz}, 1 \mathrm{H}), 1.98-1.87(\mathrm{~m}, 1 \mathrm{H}), 1.81(\mathrm{~s}, 1 \mathrm{H}), 1.63(\mathrm{~s}, 1 \mathrm{H}), 1.31$ (dd, $J=10.2,4.7 \mathrm{~Hz}, 2 \mathrm{H}), 1.22$ (ddd, $J=11.7,8.6,5.1 \mathrm{~Hz}, 4 \mathrm{H}), 1.12(\mathrm{~s}, 3 \mathrm{H}), 1.07$ (dd, $J=8.5,4.2 \mathrm{~Hz}$, $6 \mathrm{H}), 0.97(\mathrm{dd}, J=6.3,4.0 \mathrm{~Hz}, 2 \mathrm{H}), 0.89(\mathrm{~s}, 3 \mathrm{H}), 0.83(\mathrm{t}, J=7.2 \mathrm{~Hz}, 3 \mathrm{H}), 0.78$ (dd, $J=8.4,2.0 \mathrm{~Hz}, 2 \mathrm{H})$, $0.74-0.70(\mathrm{~m}, 3 \mathrm{H}), 0.62(\mathrm{td}, J=9.5,5.3 \mathrm{~Hz}, 1 \mathrm{H}) .{ }^{13} \mathrm{C}$ NMR $\left(101 \mathrm{MHz}, \mathrm{CDCl}_{3}\right) \delta 159.0,158.9,139.2$, $138.2,136.8,136.5,127.6,127.4,116.1,114.8,114.7,114.5,113.8,113.7,71.6,71.1,55.3,55.3,42.9$, $42.8,33.9,33.8,29.2,29.0,28.9,28.4,26.6,25.4,23.0,22.9,22.8,22.6,14.2,14.1,13.1,12.5$. HRMS (APCI) calcd. for $\mathrm{C}_{19} \mathrm{H}_{27} \mathrm{O}\left[(\mathrm{M}+\mathrm{H})-\mathrm{H}_{2} \mathrm{O}\right] 271.2056$ found: 271.2077 .

(S)-((1R， 2R，3S)-2-butyl-2,3-dimethylcyclopropyl)(4-methoxyphenyl)methanol (1r): $\mathrm{R}_{\mathrm{f}}=0.4$ (ether/hexane $=20: 80$ ), colourless syrupy oil, $68 \%$ yield (over two steps-Oxidation and reduction), $1: 1$ dr. ${ }^{1} \mathrm{H}$ NMR (400 MHz, $\left.\mathrm{CDCl}_{3}\right) \delta 7.28-7.22(\mathrm{~m}, 4 \mathrm{H}), 6.83-6.76(\mathrm{~m}, 4 \mathrm{H}), 4.20(\mathrm{~s}, 1 \mathrm{H}), 4.18(\mathrm{~s}, 1 \mathrm{H})$, $3.72(\mathrm{~s}, 3 \mathrm{H}), 1.74(\mathrm{~s}, 2 \mathrm{H},-\mathrm{OH}), 1.58(\mathrm{dd}, J=12.9,10.2 \mathrm{~Hz}, 1 \mathrm{H}), 1.45(\mathrm{ddd}, J=9.8,4.8,2.7 \mathrm{~Hz}, 1 \mathrm{H})$, $1.32-1.17(\mathrm{~m}, 8 \mathrm{H}), 1.06(\mathrm{~d}, J=6.2 \mathrm{~Hz}, 3 \mathrm{H}), 0.99(\mathrm{~s}, 3 \mathrm{H}), 0.93-0.83(\mathrm{~m}, 10 \mathrm{H}), 0.79(\mathrm{dd}, J=7.9,6.0$ $\mathrm{Hz}, 4 \mathrm{H}), 0.55$ (ddd, $J=19.6,9.6,5.4 \mathrm{~Hz}, 1 \mathrm{H}), 0.47-0.39(\mathrm{~m}, 1 \mathrm{H}) \cdot{ }^{13} \mathrm{C}$ NMR $\left(101 \mathrm{MHz}, \mathrm{CDCl}_{3}\right) \delta$ 158.8 , 137.2, 137.1, 127.4, 127.0, 113.7, 74.9, 74.5, 55.3, 40.6, 40.2 , 36.2, 35.8, 29.3, 29.2, 24.6, 24.5, $23.2,23.1,23.99,22.88,19.0,18.7,14.3,14.2,13.9,13.2$. HRMS (APCI) calcd. for $\mathrm{C}_{17} \mathrm{H}_{25} \mathrm{O}[(\mathrm{M}+\mathrm{H})-$ $\left.\mathrm{H}_{2} \mathrm{O}\right]: 245.1900$ found: 245.1889 .

(S)-((1R, 2R)-2-butyl-2-methylcyclopropyl)(4-methoxyphenyl)methanol (1s): Rf $=0.3$ (ether/hexane = 15:85), colourless syrupy oil, 78\% yield (over two steps- Oxidation and reduction), 1:1 dr-crude NMR, (isolated as a 1:0.7 dr., may be manual error in isolating separately and combined them). ${ }^{1} \mathrm{H}$ NMR (400 $\left.\mathrm{MHz}, \mathrm{CDCl}_{3}\right) \delta 7.26(\mathrm{dd}, J=10.3,8.1 \mathrm{~Hz}, 4 \mathrm{H}), 6.80(\mathrm{dd}, J=8.7,2.0 \mathrm{~Hz}, 4 \mathrm{H}), 4.12(\mathrm{t}, J=9.1 \mathrm{~Hz}, 2 \mathrm{H})$, $3.72(\mathrm{~d}, J=3.2 \mathrm{~Hz}, 6 \mathrm{H}), 1.25$ (ddd, $J=21.5,12.4,6.1 \mathrm{~Hz}, 4 \mathrm{H}), 1.17(\mathrm{~s}, 3 \mathrm{H}), 1.15-1.02(\mathrm{~m}, 8 \mathrm{H}), 0.99$ $-0.95(\mathrm{~m}, 4 \mathrm{H}), 0.95-0.86(\mathrm{~m}, 2 \mathrm{H}), 0.82(\mathrm{dd}, J=9.1,5.2 \mathrm{~Hz}, 3 \mathrm{H}), 0.73(\mathrm{t}, J=6.8 \mathrm{~Hz}, 4 \mathrm{H}), 0.54(\mathrm{dd}, J$ $=8.3,4.4 \mathrm{~Hz}, 1 \mathrm{H}), 0.42(\mathrm{dd}, J=8.8,4.7 \mathrm{~Hz}, 1 \mathrm{H}), 0.32(\mathrm{t}, J=4.9 \mathrm{~Hz}, 1 \mathrm{H}), 0.14(\mathrm{t}, J=5.0 \mathrm{~Hz}, 1 \mathrm{H}) .{ }^{13} \mathrm{C}$ NMR $\left(101 \mathrm{MHz}, \mathrm{CDCl}_{3}\right) \delta 158.9,136.9,127.3,127.1,113.8,75.5,74.9,55.3,40.9,32.3,31.9,29.1$, 28.6, 23.0, 22.8, 21.2, 20.8, 18.5, 18.3, 18.2, 17.6, 14.2, 14.1. HRMS (APCI) calcd. for $\mathrm{C}_{16} \mathrm{H}_{23} \mathrm{O}$ $\left[(\mathrm{M}+\mathrm{H})-\mathrm{H}_{2} \mathrm{O}\right] 231.1743$ found: 231.1765 .

2-((1R, 2R, 3S)-2-butyl-2-methyl-3-(2-phenylallyl)cyclopropyl)propan-2-ol (1t): Prepared according to the general procedure with (3-bromoprop-1-en-2-yl)benzene as electrophile, and the second step is direct addition of 2.5 equiv. of $\mathrm{MeMgBr} . \mathrm{R}_{\mathrm{f}}=0.5$ (ether/hexane $=15: 85$ ), colourless syrupy oil, $85 \%$ yield, single diastereomer. ${ }^{1} \mathrm{H} \mathrm{NMR}\left(400 \mathrm{MHz}, \mathrm{CDCl}_{3}\right) \delta 7.35(\mathrm{~d}, J=7.7 \mathrm{~Hz}, 2 \mathrm{H}), 7.24(\mathrm{t}, J=7.4 \mathrm{~Hz}$, $2 \mathrm{H}), 7.21-7.16(\mathrm{~m}, 1 \mathrm{H}), 5.25(\mathrm{~s}, 1 \mathrm{H}), 5.11(\mathrm{~s}, 1 \mathrm{H}), 2.83(\mathrm{dd}, J=17.0,8.3 \mathrm{~Hz}, 1 \mathrm{H}), 2.73(\mathrm{dd}, J=16.9$, $6.2 \mathrm{~Hz}, 1 \mathrm{H}), 1.28(\mathrm{~s}, 4 \mathrm{H}), 1.26(\mathrm{~s}, 4 \mathrm{H}), 1.23-1.14(\mathrm{~m}, 3 \mathrm{H}), 1.13(\mathrm{~s}, 3 \mathrm{H}), 0.99(\mathrm{~d}, J=1.4 \mathrm{~Hz}, 1 \mathrm{H}), 0.90$ $(\mathrm{d}, J=9.6 \mathrm{~Hz}, 1 \mathrm{H}), 0.80(\mathrm{t}, J=7.0 \mathrm{~Hz}, 3 \mathrm{H}), 0.72(\mathrm{dd}, J=15.3,8.8 \mathrm{~Hz}, 1 \mathrm{H}), 0.40(\mathrm{~d}, J=9.5 \mathrm{~Hz}, 1 \mathrm{H})$. ${ }^{13} \mathrm{C}$ NMR (101 MHz, $\left.\mathrm{CDCl}_{3}\right) \delta 149.9,142.3,128.2,127.3,126.2,111.9,71.9,44.9,35.7,32.9,31.8$, 30.0, 28.9, 26.3, 23.1, 22.4, 14.2, 12.5. HRMS (APCI) calcd. for $\mathrm{C}_{20} \mathrm{H}_{29}\left[(\mathrm{M}+\mathrm{H})-\mathrm{H}_{2} \mathrm{O}\right] 269.2264$ found: 269.2276 .

\section{General Procedure for Nucleophilic Substitution at the Quaternary Carbon Stereocenters}

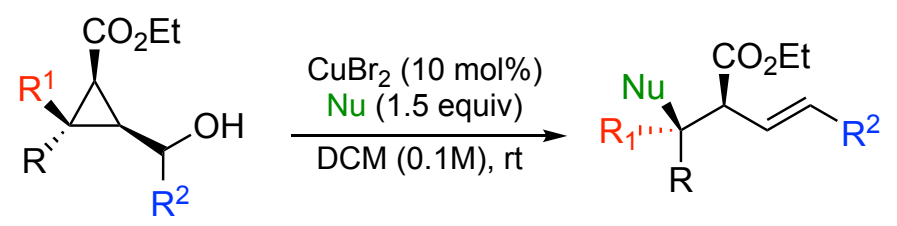

Procedure for Halogenation To a $15 \mathrm{~mL}$ oven dried (or flame dried) Schlenk tube, purged with argon, was added $\mathrm{CuBr}_{2}(10 \mathrm{~mol} \%)$ in $2 \mathrm{~mL}$ of dichloromethane and sealed with a septum. Then, a solution of cyclopropane carbinol in $1 \mathrm{~mL}$ of DCM ( $0.3 \mathrm{mmol}, 1$ equiv.) was added with a syringe followed by a dropwise addition of the nucleophile (TMSBr $/ \mathrm{TMSCl} / 48 \%$ aq. $\left.\mathrm{HBF}_{4}\right)(0.45 \mathrm{mmol}, 1.5$ equiv.) in one 
minute at room temperature. After completion of the reaction (monitored by TLC), the reaction mixture was quenched with a saturated solution of $\mathrm{NH}_{4} \mathrm{Cl}$ (saturated $\mathrm{Na}_{2} \mathrm{CO}_{3}$ soln. in the case of fluorination) and diluted with DCM. The organic layers were extracted with DCM ( 3 X $20 \mathrm{~mL})$ dried over $\mathrm{MgSO}_{4}$, filtered and concentrated under vacuum to give the halogenated product, further purified by column chromatography when needed.

Procedure for carboxylation/hydroxylation In a $15 \mathrm{~mL}$ dry Schlenk tube containing trichloroacetic acid (1.5 equiv.) in $2 \mathrm{~mL}$ of DCM, was added the cyclopropane carbinol derivative ( $0.3 \mathrm{mmol}, 1.0$ equiv.) in $1 \mathrm{~mL}$ DCM. After completion of the reaction (monitored by TLC), the reaction mixture was quenched with an aqueous saturated solution of $\mathrm{NH}_{4} \mathrm{Cl}(5 \mathrm{~mL})$ and diluted with DCM. The organic layers were washed with saturated solution of $\mathrm{Na}_{2} \mathrm{CO}_{3}(10 \mathrm{~mL})$ and extracted with DCM $(3 \mathrm{X} 20 \mathrm{~mL})$ dried over $\mathrm{MgSO}_{4}$, filtered and concentrated under vacuum to give the carboxylated product, further purified by column chromatography when needed.

Scale of the reaction: tested up to $5 \mathrm{mmol}$. 


\section{Optimization Table}<smiles>CCOC(=O)C1C(C(O)P)C1(C)Br</smiles><smiles>CCOC(=O)C(/C=C/c1ccccc1)C(C)(C#N)C(=O)OCCO[Mg]</smiles>

2

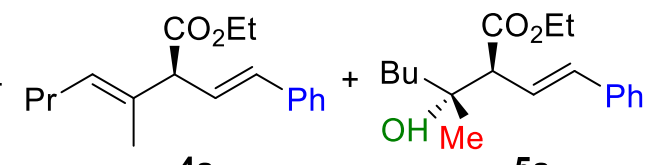

$5 a$

\begin{tabular}{|c|c|c|c|c|c|}
\hline S. No & $\begin{array}{l}\text { Halogen source } \\
\text { (equiv.) }\end{array}$ & $\begin{array}{l}\text { Catalyst } \\
\text { (mol\%) }\end{array}$ & $\begin{array}{l}\text { Solvent } \\
\text { (M) }\end{array}$ & $\begin{array}{c}\text { Temp/h } \\
{ }^{\circ} \mathrm{C}\end{array}$ & $\begin{array}{l}\text { Product (\%) } \\
2+4 a+5 a\end{array}$ \\
\hline 1 & $48 \% \mathrm{HBr}(1.5)$ & $48 \% \mathrm{HBr}$ & DCM (0.1M) & $\mathrm{rt} / 12 \mathrm{~h}$ & $15(d r .98: 2)+10+08$ \\
\hline 2 & $48 \% \mathrm{HBr}(1.5)$ & PTSA. $\mathrm{H}_{2} \mathrm{O}$ & $\mathrm{DCM}(0.1 \mathrm{M})$ & $\mathrm{rt} / 12 \mathrm{~h}$ & $30(\mathrm{dr} .95: 5)+15+10$ \\
\hline 3 & TMSBr (1.5) & PTSA. $\mathrm{H}_{2} \mathrm{O}$ & DCM (0.1M) & $\mathrm{rt} / 12 \mathrm{~h}$ & $45(d r .95: 5)+10+04$ \\
\hline 4 & TBAB (1.5) & PTSA. $\mathrm{H}_{2} \mathrm{O}$ & DCM (0.1M) & $\mathrm{rt} / 12 \mathrm{~h}$ & $00+45+15$ \\
\hline 5 & $T M S B r(1.5)$ & $\operatorname{lnBr}_{3}(10)$ & $D C M(0.1 M)$ & $r t / 20 \min$ & $84(d r .98: 2)+00+02$ \\
\hline 6 & TMSBr (1.5) & $\operatorname{lnBr}_{3}(10)$ & $\mathrm{DCM}(0.1 \mathrm{M})$ & $0 / 35 \min$ & $85(\mathrm{dr} .98: 2)+00+02$ \\
\hline 7 & TMSBr (1.5) & - & $\operatorname{DCM}(0.1 \mathrm{M})$ & $\mathrm{rt} / 24 \mathrm{~h}$ & $45(d r .98: 2)+35+00$ \\
\hline 8 & TMSBr (1.5) & $\operatorname{lnCl}_{3}(10)$ & $\operatorname{DCM}(0.1 \mathrm{M})$ & $\mathrm{rt} / 25 \mathrm{~min}$ & $84(d r .98: 2)+00+00$ \\
\hline 9 & TMSBr (1.5) & $\mathrm{FeCl}_{3}(10)$ & $\operatorname{DCM}(0.1 \mathrm{M})$ & $\mathrm{rt} / 25 \mathrm{~min}$ & $80(d r .75: 25)+10+02$ \\
\hline 10 & $\operatorname{TMSBr}(1.5)$ & $\mathrm{CuBr}_{2}(10)$ & $D C M(0.1 M)$ & $r t / 2 h$ & $83(d r .98: 2)+00+02$ \\
\hline 11 & TMSBr (1.5) & $\mathrm{Bi}(\mathrm{OTf})_{3}(10)$ & $\operatorname{DCM}(0.1 \mathrm{M})$ & $\mathrm{rt} / 25 \mathrm{~min}$ & $70(d r .65: 35)+15+00$ \\
\hline 12 & $\mathrm{TMSBr}(1.5)$ & $\mathrm{FeCl}_{2}(10)$ & $\operatorname{DCM}(0.1 \mathrm{M})$ & $\mathrm{rt} / 25 \mathrm{~min}$ & $75(\mathrm{dr} .85: 15)+10+00$ \\
\hline 13 & TMSBr (1.5) & $\mathrm{Cu}(\mathrm{OTf})_{3}(10)$ & DCM (0.1M) & $r t / 2 h$ & $54(\mathrm{dr} .95: 5)+00+02$ \\
\hline 14 & TMSCI (1.5) & $\mathrm{CuBr}_{2}(10)$ & DCM (0.1M) & $\mathrm{rt} / 2 \mathrm{~h}$ & $76(d r .98: 2)+05+00$ \\
\hline 15 & TFA (1.5) & - & DCM (0.1M) & $\mathrm{rt} / 2 \mathrm{~h}$ & $65(d r .98: 2)+11+10$ \\
\hline 16 & TCA (1.5) & - & DCM (0.1M) & $\mathrm{rt} / 2 \mathrm{~h}$ & $67(d r .98: 2)+10+10$ \\
\hline 17 & $48 \%$ aq.HBF4 (2) & $\mathrm{CuBr}_{2}(10)$ & $D C M(0.1 M)$ & $r t / 2 h$ & $52(d r .98: 2)+05+20$ \\
\hline 18 & $48 \%$ aq. HBF4 (2) & - & DCM (0.1M) & $\mathrm{rt} / 12 \mathrm{~h}$ & $40(d r .98: 2)+10+25$ \\
\hline
\end{tabular}




\section{Optimization for the fluorination reaction}<smiles>CCOC(=O)C1C(C(O)c2ccccc2)C1(C)Br</smiles>

$1 \mathrm{a}$

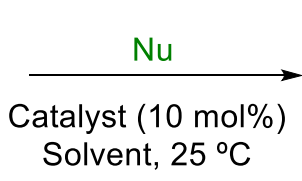

Solvent, $25^{\circ} \mathrm{C}$<smiles>CCOC(=O)C(/C=C/[PH2+]/C=C/[PH2+])/C(C)=C/C=[PH2+]</smiles>

4a<smiles>CCOC(=O)C(/C=C/c1ccccc1)C(C)=O</smiles>

$5 a$

\begin{tabular}{|c|c|c|}
\hline S.No & Reaction Conditions & $\mathrm{F} / \mathrm{OH} / \mathrm{EP}$ \\
\hline 1 & $48 \%$ aq. $\mathrm{HBF}_{4}$ (2equiv), DCM (0.1M), rt, $6 \mathrm{~h}$ & $45 / 15 / 5$ \\
\hline 2 & $48 \%$ aq. $\mathrm{HBF}_{4}$ (2equiv), $\mathrm{CuBr}(\mathrm{II})(10 \mathrm{~mol} \%), \mathrm{DCM}(0.1 \mathrm{M}), \mathrm{rt}, 1 \mathrm{~h}$ & $55 / 14 / 5$ \\
\hline 3 & $48 \%$ aq. $\mathrm{HBF}_{4}$ (1 equiv), $\mathrm{CuBr}(\mathrm{II})(10 \mathrm{~mol} \%), \mathrm{DCM}(0.1 \mathrm{M}), \mathrm{rt}, 2 \mathrm{~h}$ & $48 / 12 / 5$ \\
\hline 4 & $48 \%$ aq. $\mathrm{HBF}_{4}$ (1 equiv), DCM $(0.1 \mathrm{M}), \mathrm{rt}, 6 \mathrm{~h}$ & $46 / 14 / 5$ \\
\hline 6 & $48 \%$ aq. $\mathrm{HBF}_{4}$ (2equiv), DCM (0.1M), rt, 6h, Argon atm. & $52 / 16 / 5$ \\
\hline 7 & $48 \%$ aq. $\mathrm{HBF}_{4}$ (2equiv), $\mathrm{DCM}(0.1 \mathrm{M}),-15^{\circ} \mathrm{C}, 7 \mathrm{~h}$ & $25 / 20 / 41$ \\
\hline 8 & $\mathrm{NH}_{4} \mathrm{BF}_{4}$ (1 equiv), DCM (0.1M), RT, 6h & $\mathrm{nr}$ \\
\hline 9 & $\mathrm{HBF}_{4} \cdot \mathrm{Et}_{2} \mathrm{O}$ (2equiv), DCM (0.1M), RT, $2 \mathrm{~h}$ & $00 / 00 /(47+45)$ \\
\hline 10 & $\mathrm{HBF}_{4} \cdot \mathrm{Et}_{2} \mathrm{O}$ (1 equiv), DCM $(0.1 \mathrm{M}),-30^{\circ} \mathrm{C}, 4 \mathrm{~h}$ & $26 / 4 / 35$ \\
\hline 11 & $\mathrm{KF}$ (2 equiv.), $\mathrm{CuBr}(\mathrm{II})(10 \mathrm{~mol} \%), \mathrm{DCM}(0.1 \mathrm{M}), \mathrm{rt}, 12 \mathrm{~h}$ & No reaction \\
\hline 12 & TBAF(1.2equiv), $\mathrm{CuBr}(\mathrm{II})(10 \mathrm{~mol} \%), \mathrm{DCM}(0.1 \mathrm{M}), \mathrm{rt}, 1 \mathrm{~h}$ & $\begin{array}{c}\text { Complex } \\
\text { mixture }\end{array}$ \\
\hline 13 & $\operatorname{TBAF}(1.2 \mathrm{equiv}), \mathrm{CuBr}(\mathrm{II})(10 \mathrm{~mol} \%), \mathrm{DCM}(0.1 \mathrm{M})$, at $0^{\circ} \mathrm{C}, 3 \mathrm{~h}$ & $\begin{array}{l}\text { Complex } \\
\text { mixture }\end{array}$ \\
\hline
\end{tabular}

NMR Yield - After work-up, 1 equiv. of $p$-methoxy acetophenone was added into the round bottom flask containing the combined organic layers from the work-up. Either an aliquot from the round bottom flask was taken, evaporated and then submitted to NMR or the organic layer was completely evaporated and the residue was dissolved in an appropriate amount of solvent $\left(\mathrm{CDCl}_{3}\right)$ to record the NMR. The aromatic protons of p-methoxy acetophenone and the olefinic protons of our product were well distinguished and the integrations could serve as inrenal standard (sample spectrum see at SI-19).

\section{Determination of Diastereomeric ratio}

The diastereomeric ratios were determined by ${ }^{1} \mathrm{H}$ NMR spectrum. As can be seen in scheme 2 , the two diastereomers $\mathbf{2} \mathbf{m}$ and $\mathbf{2 n}$ were independently prepared and analysis of their NMR $\left({ }^{1} \mathrm{H},{ }^{13} \mathrm{C}\right.$ and $\left.{ }^{19} \mathrm{~F}\right)$ shows major differences. For all other addition of nucleophiles, allylic hydrogens of the two diastereomers are well distinguished by ${ }^{1} \mathrm{H}$ NMR (see sample spectrum at SI-20).

\section{Characterization data of new compounds}

Ethyl (2R, 3R)-3-bromo-3-methyl-2-((E)-styryl)heptanoate (2a): $\mathrm{R}_{\mathrm{f}}=0.6$ (ether/hexane $=05: 95$ ), colourless thick oil, 78\% yield, 95:5 dr determined by ${ }^{1} \mathrm{H}-\mathrm{NMR} .{ }^{1} \mathrm{H}$ NMR $\left(400 \mathrm{MHz}, \mathrm{CDCl}_{3}\right) \delta 7.35-$ $7.31(\mathrm{~m}, 2 \mathrm{H}), 7.26(\mathrm{t}, J=7.3 \mathrm{~Hz}, 2 \mathrm{H}), 7.22-7.18(\mathrm{~m}, 1 \mathrm{H}), 6.49(\mathrm{~d}, J=15.8 \mathrm{~Hz}, 1 \mathrm{H}), 6.25(\mathrm{dd}, J=$ $15.8,9.6 \mathrm{~Hz}, 1 \mathrm{H}), 4.14$ (dddd, $J=18.0,10.8,7.1,3.7 \mathrm{~Hz}, 2 \mathrm{H}), 3.58(\mathrm{~d}, J=9.6 \mathrm{~Hz}, 1 \mathrm{H}), 1.85-1.79$ (m, $5 \mathrm{H}), 1.48$ (ddd, $J=16.8,9.1,6.0 \mathrm{~Hz}, 2 \mathrm{H}), 1.30-1.19(\mathrm{~m}, 5 \mathrm{H}), 0.87(\mathrm{t}, J=5.5 \mathrm{~Hz}, 3 \mathrm{H}) .{ }^{13} \mathrm{C} \mathrm{NMR}(101$ $\left.\mathrm{MHz}, \mathrm{CDCl}_{3}\right) \delta 171.0,136.3,135.1,128.7,128.1,126.6,124.0,70.6,61.5,61.0,43.4,28.5,27.8,22.7$, 14.2,14.1. HRMS (APCI) calcd. for $\mathrm{C}_{18} \mathrm{H}_{26} \mathrm{BrO}_{2}[\mathrm{M}+\mathrm{H}]$ : 353.1116 found: 353.1131 . 
Ethyl (2R, 3R)-3-bromo-2-((E)-4-bromostyryl)-3-methylheptanoate $(\mathbf{2 b}): \mathrm{R}_{\mathrm{f}}=0.5$ (ether/hexane $=$ 05:95), colourless thick oil, 80\% yield, 95:5 dr determined by ${ }^{1} \mathrm{H}-\mathrm{NMR} .{ }^{1} \mathrm{H}$ NMR $\left(400 \mathrm{MHz}, \mathrm{CDCl}_{3}\right)$ $\delta 7.37(\mathrm{~d}, J=8.4 \mathrm{~Hz}, 2 \mathrm{H}), 7.18(\mathrm{~d}, J=8.2 \mathrm{~Hz}, 2 \mathrm{H}), 6.41(\mathrm{~d}, J=15.9 \mathrm{~Hz}, 1 \mathrm{H}), 6.24(\mathrm{dd}, J=15.8,9.5$ $\mathrm{Hz}, 1 \mathrm{H}), 4.19-4.05(\mathrm{~m}, 2 \mathrm{H}), 3.54(\mathrm{~d}, J=9.5 \mathrm{~Hz}, 1 \mathrm{H}), 2.21-2.09(\mathrm{~m}, 4 \mathrm{H}), 1.79(\mathrm{~s}, 3 \mathrm{H}), 1.29-1.15$ $(\mathrm{m}, 5 \mathrm{H}), 0.85$ (t, $J=7.3 \mathrm{~Hz}, 3 \mathrm{H}) .{ }^{13} \mathrm{C} \mathrm{NMR}\left(101 \mathrm{MHz}, \mathrm{CDCl}_{3}\right) \delta 170.8,135.3,133.9,131.8,128.1$, 124.9, 121.9, 70.6, 61.4, 61.1, 43.3, 28.6, 27.8, 22.7, 14.2, 14.1. HRMS (APCI) calcd. for $\mathrm{C}_{18} \mathrm{H}_{25} \mathrm{Br}_{2} \mathrm{O}_{2}$ $[\mathrm{M}+\mathrm{H}]: 431.0216$ found: 431.0239 .

Ethyl (2R, 3R)-2-((E)-2-(benzo[d][1,3] dioxol-5-yl)vinyl)-3-bromo-3-methylheptanoate $(2 \mathrm{c}): \mathrm{R}_{\mathrm{f}}=0.4$ (ether/hexane $=05: 95$ ), colourless thick oil, 75\% yield, 95:5 dr determined by ${ }^{1} \mathrm{H}-\mathrm{NMR} .{ }^{1} \mathrm{H}$ NMR (400 $\left.\mathrm{MHz}, \mathrm{CDCl}_{3}\right) \delta 6.87(\mathrm{~d}, J=1.0 \mathrm{~Hz}, 1 \mathrm{H}), 6.75(\mathrm{~d}, J=8.1 \mathrm{~Hz}, 1 \mathrm{H}), 6.69(\mathrm{~d}, J=8.0 \mathrm{~Hz}, 1 \mathrm{H}), 6.39(\mathrm{~d}, J=$ $15.8 \mathrm{~Hz}, 1 \mathrm{H}), 6.06(\mathrm{dd}, J=15.8,9.6 \mathrm{~Hz}, 1 \mathrm{H}), 5.89(\mathrm{~s}, 2 \mathrm{H}), 4.21-4.03(\mathrm{~m}, 2 \mathrm{H}), 3.53(\mathrm{~d}, J=9.6 \mathrm{~Hz}$, $1 \mathrm{H}), 1.87-1.74(\mathrm{~m}, 5 \mathrm{H}(3+2)), 1.47(\mathrm{~d}, J=7.4 \mathrm{~Hz}, 2 \mathrm{H}), 1.24(\mathrm{ddt}, J=17.2,12.0,8.8 \mathrm{~Hz}, 5 \mathrm{H}), 0.85(\mathrm{t}$, $J=7.3 \mathrm{~Hz}, 3 \mathrm{H}) .{ }^{13} \mathrm{C}$ NMR $\left(101 \mathrm{MHz}, \mathrm{CDCl}_{3}\right) \delta 171.1,148.1,147.6,134.7,130.8,122.1,121.5,108.3$, 105.8, 101.2, 70.8, 61.4, 61.0, 43.3, 28.4, 27.8, 22.7, 14.2, 14.1 HRMS (APCI) calcd. for $\mathrm{C}_{19} \mathrm{H}_{26} \mathrm{BrO}_{4}$ [M+H]: 397.1014 found: 397.1000 .

Ethyl (2R, 3S)-3-bromo-3-methyl-4-oxo-2-((E)-styryl)nonanoate (2d): $\mathrm{R}_{\mathrm{f}}=0.6$ (ether/hexane $=05: 95$ ), colourless thick oil, 68\% yield, 95:5 dr determined by ${ }^{1} \mathrm{H}-\mathrm{NMR} .{ }^{1} \mathrm{H}$ NMR $\left(400 \mathrm{MHz}, \mathrm{CDCl}_{3}\right) \delta 7.32$ $(\mathrm{d}, J=7.4 \mathrm{~Hz}, 2 \mathrm{H}), 7.25(\mathrm{t}, J=7.5 \mathrm{~Hz}, 2 \mathrm{H}), 7.22-7.15(\mathrm{~m}, 1 \mathrm{H}), 6.48(\mathrm{~d}, J=15.8 \mathrm{~Hz}, 1 \mathrm{H}), 6.23(\mathrm{dd}, J$ $=15.8,9.6 \mathrm{~Hz}, 1 \mathrm{H}), 4.19-4.05(\mathrm{~m}, 2 \mathrm{H}), 3.57(\mathrm{~d}, J=9.6 \mathrm{~Hz}, 1 \mathrm{H}), 1.86-1.77(\mathrm{~m}, 5 \mathrm{H}), 1.49(\mathrm{dd}, J=$ $15.9,9.2 \mathrm{~Hz}, 2 \mathrm{H}), 1.28-1.19(\mathrm{~m}, 9 \mathrm{H}), 0.80(\mathrm{t}, J=5.7 \mathrm{~Hz}, 3 \mathrm{H}) .{ }^{13} \mathrm{C} \mathrm{NMR}\left(101 \mathrm{MHz}, \mathrm{CDCl}_{3}\right) \delta 171.0$, 136.3, 135.1, 128.7, 128.1, 126.6, 124.0,70.7, 61.5, 61.0, 43.7, 31.6, 29.2, 28.5, 25.5, 22.6, 14.2, 14.1 . HRMS (APCI) calcd. for $\mathrm{C}_{20} \mathrm{H}_{28} \mathrm{BrO}_{3}[\mathrm{M}+\mathrm{H}]$ : 395.1222found: 395.1212 .

Diethyl-2,2'-((1E,1'E)-1,4-phenylenebis(ethene-2,1-diyl))(2R， 2'R， 3R， 3'R)-bis(3-bromo-3 methylheptanoate) $(\mathbf{2 e}): \mathrm{R}_{\mathrm{f}}=0.4$ (ether/hexane $=15: 85$ ), colourless thick oil, 68\% yield, 95:5 $\mathrm{dr}$ determined by ${ }^{1} \mathrm{H}-\mathrm{NMR} .{ }^{1} \mathrm{H}$ NMR $\left(400 \mathrm{MHz}, \mathrm{CDCl}_{3}\right) \delta 7.27(\mathrm{~s}, 4 \mathrm{H}), 6.46(\mathrm{~d}, J=15.8 \mathrm{~Hz}, 2 \mathrm{H}), 6.24$ (dd, $J=15.8,9.5 \mathrm{~Hz}, 2 \mathrm{H}), 4.23-4.05(\mathrm{~m}, 4 \mathrm{H}), 3.56(\mathrm{~d}, J=9.5 \mathrm{~Hz}, 2 \mathrm{H}), 1.85-1.77(\mathrm{~m}, 10 \mathrm{H}), 1.48$ $(\mathrm{dd}, J=15.3,7.9 \mathrm{~Hz}, 4 \mathrm{H}), 1.30-1.19(\mathrm{~m}, 10 \mathrm{H}), 0.85$ (t, $J=7.3 \mathrm{~Hz}, 6 \mathrm{H}) \cdot{ }^{13} \mathrm{C}$ NMR $\left(101 \mathrm{MHz}, \mathrm{CDCl}_{3}\right)$ $\delta 171.0,136.1,134.6,126.9,124.2,70.6,61.5,61.0,43.3,28.5,27.7,22.7,14.2,14.1$. HRMS (APCI) calcd. for $\mathrm{C}_{30} \mathrm{H}_{45} \mathrm{Br}_{2} \mathrm{O}_{4}[\mathrm{M}+\mathrm{H}]: 627.1685$ found: 627.1685 .

Ethyl (2R, 3R)-3-chloro-3-methyl-2-((E)-styryl)heptanoate (2f): $R_{\mathrm{f}}=0.5$ (ether/hexane $=05: 95$ ), colourless thick oil, 70\% yield, 95:5 dr determined by ${ }^{1} \mathrm{H}-\mathrm{NMR} .{ }^{1} \mathrm{H} \mathrm{NMR}\left(400 \mathrm{MHz}, \mathrm{CDCl}_{3}\right) \delta 7.37-$ 7.29 (m, 2H), 7.25 (t, $J=7.4 \mathrm{~Hz}, 2 \mathrm{H}), 7.21-7.17(\mathrm{~m}, 1 \mathrm{H}), 6.47(\mathrm{~d}, J=15.9 \mathrm{~Hz}, 1 \mathrm{H}), 6.23$ (dd, $J=$ 15.9, 9.7 Hz, 1H), 4.12 (dddd, $J=14.1,8.6,7.2,3.7 \mathrm{~Hz}, 2 \mathrm{H}), 3.49$ (d, $J=9.6 \mathrm{~Hz}, 1 \mathrm{H}), 1.84-1.69$ (m, 2H), $1.62(\mathrm{~s}, 3 \mathrm{H}), 1.50-1.41(2,4 \mathrm{H}), 1.27-1.18(\mathrm{~m}, 5 \mathrm{H}), 0.85(\mathrm{t}, J=7.3 \mathrm{~Hz}, 3 \mathrm{H}) .{ }^{13} \mathrm{C}$ NMR $(101$ $\left.\mathrm{MHz}, \mathrm{CDCl}_{3}\right) \delta 171.1,136.4,135.1,128.7,128.1,126.6,123.7,73.4,60.9,60.7,42.3,26.9,26.4,22.8$, 14.2, 14.1. HRMS (APCI) calcd. for $\mathrm{C}_{18} \mathrm{H}_{26} \mathrm{ClO}_{2}[\mathrm{M}+\mathrm{H}]$ : 309.1621 found: 309.1608.

Ethyl (2R, 3R)-3-chloro-3-methyl-2-((E)-2-(naphthalen-1-yl)vinyl)heptanoate $\quad(\mathbf{2 g}): \mathrm{R}_{\mathrm{f}}=0.5$ (ether/hexane $=05: 95)$, colourless thick oil, 60\% yield, 95:5 dr determined by ${ }^{1} \mathrm{H}-\mathrm{NMR} .{ }^{1} \mathrm{H}$ NMR $(400$ $\left.\mathrm{MHz}, \mathrm{CDCl}_{3}\right) \delta 7.98(\mathrm{~d}, J=8.2 \mathrm{~Hz}, 1 \mathrm{H}), 7.82-7.75(\mathrm{~m}, 1 \mathrm{H}), 7.71(\mathrm{~d}, J=8.2 \mathrm{~Hz}, 1 \mathrm{H}), 7.51(\mathrm{~d}, J=7.2$ $\mathrm{Hz}, 1 \mathrm{H}), 7.47-7.32(\mathrm{~m}, 3 \mathrm{H}), 7.23(\mathrm{~d}, J=15.6 \mathrm{~Hz}, 1 \mathrm{H}), 6.26(\mathrm{dd}, J=15.6,9.7 \mathrm{~Hz}, 1 \mathrm{H}), 4.25-4.08$ $(\mathrm{m}, 2 \mathrm{H}), 3.65(\mathrm{~d}, J=9.7 \mathrm{~Hz}, 1 \mathrm{H}), 1.90-1.78(\mathrm{~m}, 2 \mathrm{H}), 1.66(\mathrm{~s}, 3 \mathrm{H}), 1.58-1.46(\mathrm{~m}, 2 \mathrm{H}), 1.30-1.20$ $(\mathrm{m}, 5 \mathrm{H}), 0.86(\mathrm{t}, J=7.3 \mathrm{~Hz}, 3 \mathrm{H}) .{ }^{13} \mathrm{C}$ NMR $\left(101 \mathrm{MHz}, \mathrm{CDCl}_{3}\right) \delta 171.0,134.2,133.6,132.6,131.1$, 128.7, 128.4, 127.0, 126.3, 125.9, 125.7, 124.3, 123.7, 73.4, 61.0, 42.3, 27.2, 26.5, 22.9, 14.3, 14.1 . HRMS (APCI) calcd. for $\mathrm{C}_{22} \mathrm{H}_{28} \mathrm{ClO}_{2}[\mathrm{M}+\mathrm{H}]: 359.1778$ found: 359.1772 .

Ethyl (2R, 3R)-5-(benzyloxy)-3-chloro-3-methyl-2-((E)-styryl)pentanoate (2h): $\mathrm{R}_{\mathrm{f}}=0.5$ (ether/hexane $=10: 90)$, colourless thick oil, $65 \%$ yield, $95: 5 \mathrm{dr}$ determined by $1 \mathrm{H}-\mathrm{NMR} .{ }^{1} \mathrm{H}$ NMR $\left(400 \mathrm{MHz}, \mathrm{CDCl}_{3}\right)$ $\delta 7.29-7.15(\mathrm{~m}, 10 \mathrm{H}), 6.46(\mathrm{~d}, J=15.9 \mathrm{~Hz}, 1 \mathrm{H}), 6.20(\mathrm{dd}, J=15.8,9.6 \mathrm{~Hz}, 1 \mathrm{H}), 4.44(\mathrm{~s}, 2 \mathrm{H}), 4.16-$ $4.04(\mathrm{~m}, 2 \mathrm{H}), 3.77-3.63(\mathrm{~m}, 2 \mathrm{H}), 3.55(\mathrm{~d}, J=9.6 \mathrm{~Hz}, 1 \mathrm{H}), 2.13(\mathrm{dt}, J=10.3,6.7 \mathrm{~Hz}, 2 \mathrm{H}), 1.66(\mathrm{~s}$, $3 \mathrm{H}), 1.20(\mathrm{t}, J=7.1 \mathrm{~Hz}, 3 \mathrm{H}) .{ }^{13} \mathrm{C}$ NMR $\left(101 \mathrm{MHz}, \mathrm{CDCl}_{3}\right) \delta 170.9,138.3,136.3,135.8,128.7,128.5$, $128.1,127.8,127.7,126.7,123.2,73.3,72.0,66.9,61.0(\mathrm{~d}, J=2.9 \mathrm{~Hz}), 41.7,27.3,14.3$. HRMS (APCI) calcd. for $\mathrm{C}_{23} \mathrm{H}_{28} \mathrm{ClO}_{3}[\mathrm{M}+\mathrm{H}]$ : 387.1727 found: 387.1731 . 
(2R, 3R)-3-chloro-3-methyl-2-((E)-styryl)heptan-1-ol (2i): $\mathrm{R}_{\mathrm{f}}=0.4$ (ether/hexane $\left.=20: 80\right)$, colourless thick oil, 90\% yield, 98:2 dr determined by 1H-NMR. ${ }^{1} \mathrm{H}$ NMR $(400 \mathrm{MHz}, \mathrm{CDCl} 3) \delta 7.33$ (d, J = 7.2 $\mathrm{Hz}, 2 \mathrm{H}), 7.26(\mathrm{t}, \mathrm{J}=7.5 \mathrm{~Hz}, 2 \mathrm{H}), 7.18(\mathrm{dd}, \mathrm{J}=8.0,6.5 \mathrm{~Hz}, 1 \mathrm{H}), 6.49(\mathrm{~d}, \mathrm{~J}=15.9 \mathrm{~Hz}, 1 \mathrm{H}), 6.10(\mathrm{dd}, \mathrm{J}=$ 15.8, $9.6 \mathrm{~Hz}, 1 \mathrm{H}), 3.98(\mathrm{dd}, \mathrm{J}=10.8,4.0 \mathrm{~Hz}, 1 \mathrm{H}), 3.64(\mathrm{dd}, \mathrm{J}=10.8,9.0 \mathrm{~Hz}, 1 \mathrm{H}), 2.57(\mathrm{td}, \mathrm{J}=9.2,3.9$ $\mathrm{Hz}, 1 \mathrm{H}), 1.76$ (ddd, J = 15.9, 7.8, 3.9 Hz, 2H), $1.48-1.35(\mathrm{~m}, 5 \mathrm{H}), 1.25(\mathrm{dd}, \mathrm{J}=14.6,7.3 \mathrm{~Hz}, 2 \mathrm{H}), 0.84$ (t, J = 7.3 Hz, 3H). 13C NMR (101 MHz, CDCl3) $\delta 136.6,135.4,128.7,127.9,127.0,126.5,75.2,62.4$, 56.3, 43.4, 28.2, 26.5, 22.9, 14.1. HRMS (APCI) calcd. for $\mathrm{C}_{16} \mathrm{H}_{25} \mathrm{ClO}[\mathrm{M}+\mathrm{H}]: 267.1516$ found: 267.1529 .

1-((3S, 4R, E)-3-allyl-4-chloro-4-methyloct-1-en-1-yl)-4-methoxybenzene (2j): $\mathrm{R}_{\mathrm{f}}=0.7$ (ether/hexane $=05: 95)$, colourless thick oil, $90 \%$ yield, $98: 2 \mathrm{dr}$ determined by ${ }^{1} \mathrm{H}-\mathrm{NMR}$, crude product itself clean, no further purification required. ${ }^{1} \mathrm{H}$ NMR $(400 \mathrm{MHz}, \mathrm{CDCl} 3) \delta 7.22(\mathrm{~d}, \mathrm{~J}=8.7 \mathrm{~Hz}, 2 \mathrm{H}), 6.77(\mathrm{~d}, \mathrm{~J}=$ $8.8 \mathrm{~Hz}, 2 \mathrm{H}), 6.23(\mathrm{~d}, \mathrm{~J}=15.8 \mathrm{~Hz}, 1 \mathrm{H}), 5.80(\mathrm{dd}, \mathrm{J}=15.8,9.5 \mathrm{~Hz}, 1 \mathrm{H}), 5.66$ (ddt, J = 17.1, 10.1, 7.0 Hz, $1 \mathrm{H}), 4.99-4.91(\mathrm{~m}, 1 \mathrm{H}), 4.91-4.84(\mathrm{~m}, 1 \mathrm{H}), 3.72(\mathrm{~s}, 3 \mathrm{H}), 2.67-2.54(\mathrm{~m}, 1 \mathrm{H}), 2.41-2.30(\mathrm{~m}, 1 \mathrm{H})$, $2.18-2.08(\mathrm{~m}, 1 \mathrm{H}), 1.75(\mathrm{ddd}, \mathrm{J}=10.7,8.3,5.1 \mathrm{~Hz}, 2 \mathrm{H}), 1.49-1.33(\mathrm{~m}, 5 \mathrm{H}), 1.24(\mathrm{dd}, \mathrm{J}=14.6,7.3$ $\mathrm{Hz}, 2 \mathrm{H}), 0.84(\mathrm{t}, \mathrm{J}=7.3 \mathrm{~Hz}, 3 \mathrm{H}) .13 \mathrm{C}$ NMR $(101 \mathrm{MHz}, \mathrm{CDCl} 3) \delta 158.0,136.1,131.4,129.0,126.3$, $126.2,114.9,112.9,76.4,76.3,76.0,75.7,54.3,52.9,42.1,33.6,26.3,25.5,21.9,13.0$. HRMS (APCI) calcd. for $\mathrm{C}_{19} \mathrm{H}_{28} \mathrm{ClO}[\mathrm{M}+\mathrm{H}]$ : 307.1823 found: 307.1801 .

1-((3S, 4R, E)-4-chloro-3,4-dimethyloct-1-en-1-yl)-4-methoxybenzene $(\mathbf{2 k}): \mathrm{R}_{\mathrm{f}}=0.5$ (ether/hexane $=$ 05:95), colourless thick oil, 90\% yield, single isomer determined by $1 \mathrm{H}-\mathrm{NMR} .{ }^{1} \mathrm{H}$ NMR $(400 \mathrm{MHz}$, CDCl3) $\delta 7.27-7.19(\mathrm{~m}, 2 \mathrm{H}), 6.81-6.74(\mathrm{~m}, 2 \mathrm{H}), 6.27(\mathrm{~d}, \mathrm{~J}=15.8 \mathrm{~Hz}, 1 \mathrm{H}), 5.99(\mathrm{dd}, \mathrm{J}=15.8,8.8$ $\mathrm{Hz}, 1 \mathrm{H}), 3.72(\mathrm{~s}, 3 \mathrm{H}), 2.49(\mathrm{dt}, \mathrm{J}=13.6,6.8 \mathrm{~Hz}, 1 \mathrm{H}), 1.79-1.63(\mathrm{~m}, 2 \mathrm{H}), 1.48-1.32(\mathrm{~m}, 5 \mathrm{H}), 1.24$ $(\mathrm{dd}, \mathrm{J}=14.5,7.2 \mathrm{~Hz}, 2 \mathrm{H}), 1.15(\mathrm{~d}, \mathrm{~J}=6.7 \mathrm{~Hz}, 3 \mathrm{H}), 0.85(\mathrm{t}, \mathrm{J}=7.3 \mathrm{~Hz}, 3 \mathrm{H}) .{ }^{13} \mathrm{C}$ NMR $(101 \mathrm{MHz}$, $\mathrm{CDCl} 3) \delta 159.0,130.5,130.3,129.6,127.3,114.0,78.0,55.4,48.0,42.8,27.3,26.7,23.0,16.5,14.1$. HRMS (APCI) calcd. for $\mathrm{C}_{17} \mathrm{H}_{26} \mathrm{ClO}$ [M+H]: 281.1672found: 281.1684 .

(R, E)-1-(4-chloro-4-methyloct-1-en-1-yl)-4-methoxybenzene (2l): $R_{\mathrm{f}}=0.5$ (ether/hexane $\left.=05: 95\right)$, colourless thick oil, 79\% yield, single isomer determined by ${ }^{1} \mathrm{H}-\mathrm{NMR} .{ }^{1} \mathrm{H}$ NMR $(400 \mathrm{MHz}, \mathrm{CDCl} 3) \delta$ $7.24(\mathrm{~d}, \mathrm{~J}=8.7 \mathrm{~Hz}, 2 \mathrm{H}), 6.77(\mathrm{~d}, \mathrm{~J}=8.7 \mathrm{~Hz}, 2 \mathrm{H}), 6.32(\mathrm{~d}, \mathrm{~J}=15.8 \mathrm{~Hz}, 1 \mathrm{H}), 6.08$ (dt, J = 15.7, $7.2 \mathrm{~Hz}$, $1 \mathrm{H}), 3.72(\mathrm{~s}, 3 \mathrm{H}), 2.63-2.48(\mathrm{~m}, 2 \mathrm{H}), 1.69(\mathrm{td}, \mathrm{J}=10.7,5.4 \mathrm{~Hz}, 2 \mathrm{H}), 1.47(\mathrm{~s}, 3 \mathrm{H}), 1.44-1.34(\mathrm{~m}, 2 \mathrm{H})$, $1.25(\mathrm{dd}, \mathrm{J}=14.7,7.3 \mathrm{~Hz}, 2 \mathrm{H}), 0.85(\mathrm{t}, \mathrm{J}=7.3 \mathrm{~Hz}, 3 \mathrm{H}) .{ }^{13} \mathrm{C}$ NMR $(101 \mathrm{MHz}, \mathrm{CDCl} 3) \delta 158.0,131.8$, $129.1,126.3,122.2,112.9,73.1,54.3,46.6,42.6,28.7,25.8,21.8,13.0$. HRMS (APCI) calcd. for $\mathrm{C}_{16} \mathrm{H}_{24} \mathrm{ClO}[\mathrm{M}+\mathrm{H}]: 267.1516$ found: 267.1522 .

Ethyl (2R, 3R)-3-fluoro-3-methyl-2-((E)-styryl)heptanoate (2m): $R_{\mathrm{f}}=0.4$ (ether/hexane $\left.=05: 95\right)$, colourless thick oil, (solid after crystallization), 50\% yield, 98:2 dr determined by ${ }^{1} \mathrm{H}-\mathrm{NMR}$. ${ }^{1} \mathrm{H}$ NMR $\left(400 \mathrm{MHz}, \mathrm{CDCl}_{3}\right) \delta 7.32(\mathrm{~d}, J=8.2 \mathrm{~Hz}, 2 \mathrm{H}), 7.25(\mathrm{t}, J=7.4 \mathrm{~Hz}, 2 \mathrm{H}), 7.18(\mathrm{~d}, J=7.1 \mathrm{~Hz}, 1 \mathrm{H}), 6.44$ $(\mathrm{d}, J=15.9 \mathrm{~Hz}, 1 \mathrm{H}), 6.21(\mathrm{dd}, J=15.9,9.8 \mathrm{~Hz}, 1 \mathrm{H}), 4.12(\mathrm{qd}, J=7.1,2.7 \mathrm{~Hz}, 2 \mathrm{H}), 3.40(\mathrm{dd}, J=11.9$, $9.8 \mathrm{~Hz}, 1 \mathrm{H}), 1.68-1.57(\mathrm{~m}, 2 \mathrm{H}), 1.41-1.30(\mathrm{~m}, 5 \mathrm{H}), 1.28-1.18(\mathrm{~m}, 5 \mathrm{H}), 0.83(\mathrm{dd}, J=9.8,4.7 \mathrm{~Hz}$, 3H). ${ }^{13} \mathrm{C}$ NMR $\left(101 \mathrm{MHz}, \mathrm{CDCl}_{3}\right) \delta 171.1(\mathrm{~d}, J=3.7 \mathrm{~Hz}), 136.5,134.6,128.7,128.0,126.5,123.5(\mathrm{~d}$, $J=7.3 \mathrm{~Hz}), 97.4(\mathrm{~d}, J=177.4 \mathrm{~Hz}), 60.9,58.3(\mathrm{~d}, J=23.8 \mathrm{~Hz}), 38.3(\mathrm{~d}, J=22.4 \mathrm{~Hz}), 25.3(\mathrm{~d}, J=4.2$ $\mathrm{Hz}), 23.0,21.7(\mathrm{~d}, J=24.6 \mathrm{~Hz}), 14.2,14.1 .{ }^{19} \mathrm{~F}$ NMR $\left(377 \mathrm{MHz}, \mathrm{CDCl}_{3}\right) \delta$-147.67. HRMS (ESI-MS) calcd. for $\mathrm{C}_{18} \mathrm{H}_{25} \mathrm{FNaO}_{2}[(\mathrm{M}+\mathrm{Na})]: 315.1731$ found: 315.1748 .

Ethyl (2R, 3S)-3-fluoro-3-methyl-2-((E)-styryl)heptanoate (2n): $R_{f}=0.4$ (ether/hexane $=05: 95$ ), colourless thick oil, $46 \%$ yield, 98:2 dr determined by $1 \mathrm{H}-\mathrm{NMR} .{ }^{1} \mathrm{H}$ NMR $\left(400 \mathrm{MHz}, \mathrm{CDCl}_{3}\right) \delta 7.32$ $(\mathrm{d}, J=7.3 \mathrm{~Hz}, 2 \mathrm{H}), 7.24(\mathrm{t}, J=7.5 \mathrm{~Hz}, 2 \mathrm{H}), 7.20-7.15(\mathrm{~m}, 1 \mathrm{H}), 6.43(\mathrm{~d}, J=16.0 \mathrm{~Hz}, 1 \mathrm{H}), 6.27(\mathrm{dd}, J$ $=15.9,9.3 \mathrm{~Hz}, 1 \mathrm{H}), 4.11(\mathrm{qd}, J=7.1,2.4 \mathrm{~Hz}, 2 \mathrm{H}), 3.38(\mathrm{dd}, J=15.4,9.3 \mathrm{~Hz}, 1 \mathrm{H}), 1.82-1.68(\mathrm{~m}, 1 \mathrm{H})$, $1.54(\mathrm{dt}, J=16.7,5.7 \mathrm{~Hz}, 1 \mathrm{H}), 1.36(\mathrm{~d}, J=22.1 \mathrm{~Hz}, 4 \mathrm{H}), 1.21(\mathrm{t}, J=7.1 \mathrm{~Hz}, 6 \mathrm{H}), 0.83(\mathrm{t}, J=8.7,5.6$ $\mathrm{Hz}, 3 \mathrm{H}) .{ }^{13} \mathrm{C}$ NMR $\left(101 \mathrm{MHz}, \mathrm{CDCl}_{3}\right) \delta 171.1(\mathrm{~d}, J=7.3 \mathrm{~Hz}), 136.7,134.6,128.6,127.9,126.6,123.4$ (d, $J=4.8 \mathrm{~Hz}), 97.0$ (d, $J=177.7 \mathrm{~Hz}), 60.9,58.3(\mathrm{~d}, J=23.9 \mathrm{~Hz}), 37.7(\mathrm{~d}, J=22.4 \mathrm{~Hz}), 25.3(\mathrm{~d}, J=$ $4.9 \mathrm{~Hz}), 23.0,22.5,22.2,14.2,14.0 .{ }^{19} \mathrm{~F}$ NMR $\left(377 \mathrm{MHz}, \mathrm{CDCl}_{3}\right) \delta-147.83$. HRMS (ESI-MS) calcd. for $\mathrm{C}_{18} \mathrm{H}_{25} \mathrm{FNaO}_{2}$ [(M+Na)]: 315.1731 found: 315.1747 .

Ethyl (2R, 3S)-3-fluoro-3-methyl-2-((E)-2-(naphthalen-1-yl)vinyl)-4-oxononanoate $(20): R_{\mathrm{f}}=0.3$ $($ ether/hexane $=05: 95)$, colourless thick oil, 40\% yield, 98:2 dr determined by $1 \mathrm{H}-\mathrm{NMR} .{ }^{1} \mathrm{H}$ NMR (400 
$\left.\mathrm{MHz}, \mathrm{CDCl}_{3}\right) \delta 8.00-7.94(\mathrm{~m}, 1 \mathrm{H}), 7.76(\mathrm{dd}, J=7.3,2.1 \mathrm{~Hz}, 1 \mathrm{H}), 7.70(\mathrm{~d}, J=8.3 \mathrm{~Hz}, 1 \mathrm{H}), 7.51(\mathrm{~d}, J$ $=7.0 \mathrm{~Hz}, 1 \mathrm{H}), 7.45-7.34(\mathrm{~m}, 3 \mathrm{H}), 7.19(\mathrm{~d}, J=15.6 \mathrm{~Hz}, 1 \mathrm{H}), 6.24(\mathrm{dd}, J=15.6,9.8 \mathrm{~Hz}, 1 \mathrm{H}), 4.15(\mathrm{qd}$, $J=7.1,3.4 \mathrm{~Hz}, 2 \mathrm{H}), 3.56(\mathrm{dd}, J=11.8,9.9 \mathrm{~Hz}, 1 \mathrm{H}), 1.73-1.62(\mathrm{~m}, 2 \mathrm{H}), 1.46-1.36(\mathrm{~m}, 5 \mathrm{H}), 1.24(\mathrm{dt}$, $J=11.5,7.4 \mathrm{~Hz}, 5 \mathrm{H}), 0.85(\mathrm{t}, J=7.3 \mathrm{~Hz}, 3 \mathrm{H}) .{ }^{13} \mathrm{C} \mathrm{NMR}\left(101 \mathrm{MHz}, \mathrm{CDCl}_{3}\right) \delta 171.1(\mathrm{~d}, J=3.8 \mathrm{~Hz})$, 134.3, 133.6, 132.0, 131.1, 128.7, 128.3, 126.8 (d, $J=7.2 \mathrm{~Hz}), 126.2$, 125.9, 125.7, 124.2, 123.7, 97.4 (d, $J=177.7 \mathrm{~Hz}), 61.0,58.5(\mathrm{~d}, J=23.9 \mathrm{~Hz}), 38.4(\mathrm{~d}, J=22.3 \mathrm{~Hz}), 25.3(\mathrm{~d}, J=4.1 \mathrm{~Hz}), 23.0,22.0$, 21.8, 14.3, 14.1. ${ }^{19} \mathrm{~F}$ NMR (377 MHz, $\left.\mathrm{CDCl}_{3}\right) \delta-147.38$. HRMS (ESI-MS) calcd. for $\mathrm{C}_{22} \mathrm{H}_{27} \mathrm{FNaO}_{2}$ [(M+Na)]: 365.1887 found: 365.1912 .

Ethyl (2R, 3R)-2-((E)-2,4-difluorostyryl)-3-fluoro-3-methylheptanoate (2p): $R_{\mathrm{f}}=0.3$ (ether/hexane $=$ 05:95), colourless thick oil, 55\% yield, 98:2 dr determined by $1 \mathrm{H}-\mathrm{NMR} .{ }^{1} \mathrm{H}$ NMR (400 $\mathrm{MHz}, \mathrm{CDCl}_{3}$ ) $\delta 7.37(\mathrm{td}, J=8.6,6.5 \mathrm{~Hz}, 1 \mathrm{H}), 6.81-6.67(\mathrm{~m}, 2 \mathrm{H}), 6.52(\mathrm{~d}, J=16.1 \mathrm{~Hz}, 1 \mathrm{H}), 6.22(\mathrm{dd}, J=16.0,9.8$ $\mathrm{Hz}, 1 \mathrm{H}), 4.17-4.06(\mathrm{~m}, 2 \mathrm{H}), 3.40(\mathrm{dd}, J=12.6,9.9 \mathrm{~Hz}, 1 \mathrm{H}), 1.69-1.54(\mathrm{~m}, 2 \mathrm{H}), 1.42-1.33(\mathrm{~m}, 5 \mathrm{H})$, $1.31-1.17(\mathrm{~m}, 5 \mathrm{H}), 0.84(\mathrm{t}, J=7.2 \mathrm{~Hz}, 3 \mathrm{H}) \cdot{ }^{13} \mathrm{C} \mathrm{NMR}\left(101 \mathrm{MHz}, \mathrm{CDCl}_{3}\right) \delta 170.9(\mathrm{~d}, J=3.8 \mathrm{~Hz})$, $163.7(\mathrm{~d}, J=12.2 \mathrm{~Hz}), 161.3(\mathrm{dd}, J=24.2,11.9 \mathrm{~Hz}), 158.9(\mathrm{~d}, J=11.7 \mathrm{~Hz}), 128.3(\mathrm{dd}, J=9.6,5.1 \mathrm{~Hz})$, $125.9(\mathrm{~d}, J=26.6 \mathrm{~Hz}), 120.7(\mathrm{dd}, J=12.3,4.0 \mathrm{~Hz}), 111.6(\mathrm{dd}, J=21.5,3.7 \mathrm{~Hz}), 104.1(\mathrm{t}, J=25.7 \mathrm{~Hz})$, $97.2(\mathrm{~d}, J=177.9 \mathrm{~Hz}), 61.0,58.5(\mathrm{~d}, J=23.8 \mathrm{~Hz}), 38.2(\mathrm{~d}, J=22.4 \mathrm{~Hz}), 25.3(\mathrm{~d}, J=4.3 \mathrm{~Hz}), 22.9,21.8$ $(\mathrm{d}, J=24.6 \mathrm{~Hz}), 14.2,14.0 .{ }^{19} \mathrm{~F}$ NMR $\left(377 \mathrm{MHz}, \mathrm{CDCl}_{3}\right) \delta-106.78--111.17(\mathrm{~m}),-112.66--116.21$ (m), -147.81. HRMS (ESI-MS) calcd. for $\mathrm{C}_{18} \mathrm{H}_{23} \mathrm{~F}_{3} \mathrm{NaO}_{2}$ [(M+Na)]: 351.1542 found: 351.1568.

Ethyl (2R, 3S)-3-fluoro-3-methyl-4-oxo-2-((E)-styryl)nonanoate (2q): $\mathrm{R}_{\mathrm{f}}=0.4$ (ether/hexane $\left.=05: 95\right)$, colourless thick oil, 48\% yield, 98:2 dr determined by 1H-NMR. ${ }^{1} \mathrm{H}$ NMR (400 MHz, CDCl3) $\delta 7.32$ (d, $J=7.4 \mathrm{~Hz}, 2 \mathrm{H}), 7.25$ (t, $J=7.5 \mathrm{~Hz}, 2 \mathrm{H}), 7.18$ (dd, $J=7.6,6.7 \mathrm{~Hz}, 1 \mathrm{H}), 6.44(\mathrm{~d}, J=15.9 \mathrm{~Hz}, 1 \mathrm{H})$, $6.21(\mathrm{dd}, J=15.9,9.8 \mathrm{~Hz}, 1 \mathrm{H}), 4.12(\mathrm{qd}, J=7.1,2.6 \mathrm{~Hz}, 2 \mathrm{H}), 3.40(\mathrm{dd}, J=11.8,9.9 \mathrm{~Hz}, 1 \mathrm{H}), 1.69-$ $1.55(\mathrm{~m}, 2 \mathrm{H}), 1.43-1.30(\mathrm{~m}, 5 \mathrm{H}), 1.21(\mathrm{t}, J=7.1 \mathrm{~Hz}, 10 \mathrm{H}), 0.80(\mathrm{t}, J=6.8 \mathrm{~Hz}, 3 \mathrm{H}) .{ }^{13} \mathrm{C}$ NMR $(101$ $\left.\mathrm{MHz}, \mathrm{CDCl}_{3}\right) \delta 171.1(\mathrm{~d}, J=3.7 \mathrm{~Hz}), 136.5,134.6,128.6,128.0,126.6,123.5(\mathrm{~d}, J=7.3 \mathrm{~Hz}), 97.4(\mathrm{~d}$, $J=177.4 \mathrm{~Hz}), 60.9,58.3(\mathrm{~d}, J=23.8 \mathrm{~Hz}), 38.6(\mathrm{~d}, J=22.3 \mathrm{~Hz}), 31.8,29.6,23.0$ (d, $J=4.2 \mathrm{~Hz}), 22.6$, $21.7(\mathrm{~d}, J=24.6 \mathrm{~Hz}), 14.2,14.1 .{ }^{19} \mathrm{~F}$ NMR $\left(377 \mathrm{MHz}, \mathrm{CDCl}_{3}\right) \delta-147.56$. HRMS (ESI-MS) calcd. for $\mathrm{C}_{20} \mathrm{H}_{27} \mathrm{FNaO}_{3}[(\mathrm{M}+\mathrm{Na})]$ : 357.1842 found: 357.1859 .

Ethyl (2R, 3R)-5-(benzyloxy)-3-fluoro-3-methyl-2-((E)-styryl)pentanoate (2r): $R_{\mathrm{f}}=0.5$ (ether/hexane $=10: 90)$, colourless thick oil, $49 \%$ yield, $98: 2 \mathrm{dr}$ determined by ${ }^{1} \mathrm{H}-\mathrm{NMR} .{ }^{1} \mathrm{H}$ NMR $\left(400 \mathrm{MHz}, \mathrm{CDCl}_{3}\right)$ $\delta 7.33-7.14(\mathrm{~m}, 10 \mathrm{H}), 6.43(\mathrm{~d}, J=15.9 \mathrm{~Hz}, 1 \mathrm{H}), 6.18(\mathrm{dd}, J=15.9,9.7 \mathrm{~Hz}, 1 \mathrm{H}), 4.43(\mathrm{~s}, 2 \mathrm{H}), 4.11(\mathrm{tt}$, $J=7.2,3.7 \mathrm{~Hz}, 2 \mathrm{H}), 3.66-3.55(\mathrm{~m}, 2 \mathrm{H}), 3.49(\mathrm{t}, J=10.2 \mathrm{~Hz}, 1 \mathrm{H}), 2.10-1.90(\mathrm{~m}, 2 \mathrm{H}), 1.43(\mathrm{~d}, J=$ $22.2 \mathrm{~Hz}, 3 \mathrm{H}), 1.20(\mathrm{t}, J=7.1 \mathrm{~Hz}, 3 \mathrm{H}) .{ }^{13} \mathrm{C} \mathrm{NMR}\left(101 \mathrm{MHz}, \mathrm{CDCl}_{3}\right) \delta 170.9(\mathrm{~d}, J=4.3 \mathrm{~Hz}), 138.3$, 136.4, 135.2, 128.6, 128.5, 128.0, 127.8, 127.7, 126.6, 123.0 (d, $J=7.3 \mathrm{~Hz}), 96.6$ (d, $J=177.5 \mathrm{~Hz})$, $73.2,65.5(\mathrm{~d}, J=5.1 \mathrm{~Hz}), 61.0,58.4(\mathrm{~d}, J=24.0 \mathrm{~Hz}), 38.1(\mathrm{~d}, J=21.9 \mathrm{~Hz}), 22.1(\mathrm{~d}, J=24.6 \mathrm{~Hz}), 14.2$. ${ }^{19} \mathrm{~F}$ NMR $\left(377 \mathrm{MHz}, \mathrm{CDCl}_{3}\right) \delta$-146.73. HRMS (ESI-MS) calcd. for $\mathrm{C}_{23} \mathrm{H}_{27} \mathrm{FNaO}_{3}[(\mathrm{M}+\mathrm{Na})]: 393.1836$ found: 393.1859 .

Diethyl 2,2'-((1E, $\quad$ 1'E)-1,4-phenylenebis(ethene-2,1-diyl))(2R,2'R,3R,3'R)-bis(3-fluoro-3methylheptanoate) $(\mathbf{2 s}): \mathrm{R}_{\mathrm{f}}=0.4$ (ether/hexane $\left.=05: 95\right)$, white solid, 30\% yield, 98:2 dr determined by ${ }^{1} \mathrm{H}-\mathrm{NMR} .{ }^{1} \mathrm{H}$ NMR $\left(400 \mathrm{MHz}, \mathrm{CDCl}_{3}\right) \delta 7.27(\mathrm{~s}, 4 \mathrm{H}), 6.41(\mathrm{~d}, J=15.9 \mathrm{~Hz}, 2 \mathrm{H}), 6.20(\mathrm{dd}, J=15.9,9.7$ $\mathrm{Hz}, 2 \mathrm{H}), 4.12(\mathrm{ddt}, J=10.8,7.1,3.6 \mathrm{~Hz}, 4 \mathrm{H}), 3.39(\mathrm{dd}, J=12.2,9.8 \mathrm{~Hz}, 2 \mathrm{H}), 1.68-1.56(\mathrm{~m}, 4 \mathrm{H}), 1.42$ $-1.33(\mathrm{~m}, 10 \mathrm{H}), 1.28-1.18(\mathrm{~m}, 10 \mathrm{H}), 0.84(\mathrm{t}, J=7.2 \mathrm{~Hz}, 6 \mathrm{H}) \cdot{ }^{13} \mathrm{C}$ NMR $\left(101 \mathrm{MHz}, \mathrm{CDCl}_{3}\right) \delta 171.1$ $(\mathrm{d}, J=3.7 \mathrm{~Hz}), 136.1,134.1,126.8,123.6(\mathrm{~d}, J=7.2 \mathrm{~Hz}), 97.4(\mathrm{~d}, J=177.6 \mathrm{~Hz}), 61.0,58.3(\mathrm{~d}, J=23.8$ $\mathrm{Hz}), 38.3(\mathrm{~d}, J=22.3 \mathrm{~Hz}), 25.3(\mathrm{~d}, J=4.1 \mathrm{~Hz}), 23.0,21.7(\mathrm{~d}, J=24.6 \mathrm{~Hz}), 14.2,14.1 .{ }^{19} \mathrm{~F}$ NMR $(377$ $\left.\mathrm{MHz}, \mathrm{CDCl}_{3}\right) \delta$-147.65. HRMS (APCI) calcd. for $\mathrm{C}_{30} \mathrm{H}_{45} \mathrm{~F}_{2} \mathrm{O}_{4}[\mathrm{M}+\mathrm{H}]$ : 507.3286found: 506.3236.

Benzyl (2R, 3R)-3-fluoro-3-methyl-2-((E)-styryl)heptanoate $(\mathbf{2 t}): \mathrm{R}_{\mathrm{f}}=0.4$ (ether/hexane $\left.=10: 90\right)$, thick oil, 39\% yield, 98:2 dr determined by ${ }^{1} \mathrm{H}-\mathrm{NMR} .{ }^{1} \mathrm{H}$ NMR (400 MHz, $\left.\mathrm{CDCl}_{3}\right) \delta 7.33-7.20(\mathrm{~m}, 10 \mathrm{H})$, $7.19-7.13(\mathrm{~m}, 1 \mathrm{H}), 6.43(\mathrm{~d}, J=15.9 \mathrm{~Hz}, 1 \mathrm{H}), 6.22(\mathrm{dd}, J=15.9,9.7 \mathrm{~Hz}, 1 \mathrm{H}), 5.16-4.99(\mathrm{~m}, 2 \mathrm{H})$, $3.46(\mathrm{dd}, J=12.4,9.7 \mathrm{~Hz}, 1 \mathrm{H}), 1.58(\mathrm{ddd}, J=12.4,11.7,5.2 \mathrm{~Hz}, 2 \mathrm{H}), 1.41-1.28$ (m, 5H), 1.19 (dd, $J$ $=14.9,5.9 \mathrm{~Hz}, 3 \mathrm{H}), 0.80(\mathrm{t}, J=7.3 \mathrm{~Hz}, 4 \mathrm{H}) .{ }^{13} \mathrm{C}$ NMR $\left(101 \mathrm{MHz}, \mathrm{CDCl}_{3}\right) \delta 171.0(\mathrm{~d}, J=3.5 \mathrm{~Hz})$, 136.5, 135.8, 134.9, 128.7, 128.6, 128.29, 128.26, 128.0, 126.6, 123.3 (d, $J=7.3 \mathrm{~Hz}), 97.4$ (d, $J=177.8$ $\mathrm{Hz}), 66.7,58.2(\mathrm{~d}, J=23.7 \mathrm{~Hz}), 38.3(\mathrm{~d}, J=22.3 \mathrm{~Hz}), 25.3(\mathrm{~d}, J=4.2 \mathrm{~Hz}), 23.0,21.9,21.7,14.1 .{ }^{19} \mathrm{~F}$ 
NMR (377 MHz, $\left.\mathrm{CDCl}_{3}\right) \delta$-147.53. HRMS (ESI-MS) calcd. for $\mathrm{C}_{23} \mathrm{H}_{27} \mathrm{FNaO}_{2}$ [(M+Na)]: 377.1887 found: 377.1912 .

1-((3R, 4R, E)-3-allyl-4-fluoro-4-methyloct-1-en-1-yl)-4-methoxybenzene (2u): $\mathrm{R}_{\mathrm{f}}=0.7$ (ether/hexane $=05: 95)$, colourless thick oil, $65 \%$ yield, $98: 2 \mathrm{dr}$ determined by $1 \mathrm{H}-\mathrm{NMR}$, crude product itself clean, along with $8 \%$ of hydroxylated product. ${ }^{1} \mathrm{H}$ NMR $\left(400 \mathrm{MHz}, \mathrm{CDCl}_{3}\right) \delta 7.25-7.19(\mathrm{~m}, 2 \mathrm{H}), 6.80-$ $6.75(\mathrm{~m}, 2 \mathrm{H}), 6.26(\mathrm{~d}, J=15.8 \mathrm{~Hz}, 1 \mathrm{H}), 5.79(\mathrm{dd}, J=15.8,9.5 \mathrm{~Hz}, 1 \mathrm{H}), 5.75-5.63(\mathrm{~m}, 1 \mathrm{H}), 4.94(\mathrm{dd}$, $J=17.1,1.9 \mathrm{~Hz}, 1 \mathrm{H}), 4.91-4.84(\mathrm{~m}, 1 \mathrm{H}), 3.71(\mathrm{~s}, 3 \mathrm{H}), 2.46-2.37(\mathrm{~m}, 1 \mathrm{H}), 2.17(\mathrm{td}, J=10.1,3.0 \mathrm{~Hz}$, $1 \mathrm{H}), 2.10-2.00(\mathrm{~m}, 1 \mathrm{H}), 1.39-1.16(\mathrm{~m}, 6 \mathrm{H}), 1.10(\mathrm{~s}, 3 \mathrm{H}), 0.85-0.80(\mathrm{~m}, 3 \mathrm{H}) .{ }^{13} \mathrm{C} \mathrm{NMR}(101 \mathrm{MHz}$, $\left.\mathrm{CDCl}_{3}\right) \delta 157.9,136.9,131.3,129.1,127.0,126.3,126.1,114.5,112.9,112.9,73.4,54.3,52.8,39.0$, 33.0, 24.4, 23.1, 22.3, 13.1. ${ }^{19} \mathrm{~F}$ NMR (377 MHz, $\left.\mathrm{CDCl}_{3}\right) \delta$-149.62. HRMS (APCI) calcd. for $\mathrm{C}_{19} \mathrm{H}_{27} \mathrm{O}$ [(M+H)-HF]: 271.2056 found: 271.2077.

Ethyl (2R, 3R)-3-fluoro-3-methyl-2-((E)-4-phenylbut-1-en-3-yn-1-yl)heptanoate $(\mathbf{2 v}): \quad R_{\mathrm{f}}=0.5$ $($ ether/hexane $=05: 95)$, colourless thick oil, 50\% yield, 98:2 dr determined by $1 \mathrm{H}-\mathrm{NMR} .{ }^{1} \mathrm{H}$ NMR $(400$ $\left.\mathrm{MHz}, \mathrm{CDCl}_{3}\right) \delta 7.40-7.29(\mathrm{~m}, 2 \mathrm{H}), 7.23(\mathrm{dd}, J=6.8,3.9 \mathrm{~Hz}, 3 \mathrm{H}), 6.19(\mathrm{dd}, J=15.9,9.9 \mathrm{~Hz}, 1 \mathrm{H})$, $5.77(\mathrm{~d}, J=15.9 \mathrm{~Hz}, 1 \mathrm{H}), 4.11(\mathrm{q}, J=7.2 \mathrm{~Hz}, 2 \mathrm{H}), 3.40-3.28(\mathrm{~m}, 1 \mathrm{H}), 1.67-1.51(\mathrm{~m}, 2 \mathrm{H}), 1.44-$ $1.29(\mathrm{~m}, 5 \mathrm{H}), 1.22(\mathrm{dd}, J=14.4,7.2 \mathrm{~Hz}, 5 \mathrm{H}), 0.85(\mathrm{t}, J=7.2 \mathrm{~Hz}, 3 \mathrm{H}) .{ }^{13} \mathrm{C}$ NMR $\left(101 \mathrm{MHz}, \mathrm{CDCl}_{3}\right) \delta$ $170.2(\mathrm{~d}, J=4.0 \mathrm{~Hz}), 136.7$ (d, $J=7.0 \mathrm{~Hz}), 131.6,128.4,128.4,123.0,114.7,97.0$ (d, $J=178.3 \mathrm{~Hz})$, 90.3, 87.1, 61.1, 58.3 (d, $J=24.4 \mathrm{~Hz}), 38.2(\mathrm{~d}, J=22.3 \mathrm{~Hz}), 25.2(\mathrm{~d}, J=4.0 \mathrm{~Hz}), 22.9,21.7$ (d, $J=24.6$ $\mathrm{Hz}$ ), 14.2, 14.1. ${ }^{19} \mathrm{~F}$ NMR (377 MHz, $\left.\mathrm{CDCl}_{3}\right) \delta-147.39$. HRMS (ESI-MS) calcd. for $\mathrm{C}_{20} \mathrm{H}_{25} \mathrm{FNaO}_{2}$ [(M+Na)]: 339.1731 found: 339.1759 .

Ethyl (2R, 3R)-3-fluoro-3-methyl-2-((E)-4-(triisopropylsilyl)but-1-en-3-yn-1-yl)heptanoate (2w): $\mathrm{R}_{\mathrm{f}}=$ 0.5 (ether/hexane $=05: 95$ ), colourless thick oil, $42 \%$ yield, $98: 2 \mathrm{dr}$ determined by $1 \mathrm{H}-\mathrm{NMR}$. (Isolated $42 \%$ of yield along with $10 \%$ of eliminated product) ${ }^{1} \mathrm{H} \mathrm{NMR}\left(400 \mathrm{MHz}, \mathrm{CDCl}_{3}\right) \delta 6.13(\mathrm{dd}, J=15.9$, $9.8 \mathrm{~Hz}, 1 \mathrm{H}), 5.60$ (d, $J=15.9 \mathrm{~Hz}, 1 \mathrm{H}), 4.11$ (ddd, $J=14.3,7.2,2.5 \mathrm{~Hz}, 2 \mathrm{H}), 3.28$ (dd, $J=11.6,10.2$ $\mathrm{Hz}, 1 \mathrm{H}), 1.65-1.54(\mathrm{~m}, 2 \mathrm{H}), 1.37-1.27(\mathrm{~m}, 5 \mathrm{H}), 1.25-1.16(\mathrm{~m}, 5 \mathrm{H}), 1.01(\mathrm{~s}, 21 \mathrm{H}), 0.88-0.81(\mathrm{~m}$, 3H). ${ }^{13} \mathrm{C}$ NMR $\left(101 \mathrm{MHz}, \mathrm{CDCl}_{3}\right) \delta 170.3(\mathrm{~d}, J=4.1 \mathrm{~Hz}), 137.2(\mathrm{~d}, J=7.0 \mathrm{~Hz}), 115.1,104.3,97.1(\mathrm{~d}$, $J=178.2 \mathrm{~Hz}), 92.0,61.1,58.2(\mathrm{~d}, J=24.3 \mathrm{~Hz}), 38.2(\mathrm{~d}, J=22.3 \mathrm{~Hz}), 25.2(\mathrm{~d}, J=4.0 \mathrm{~Hz}), 22.9,21.7$ $(\mathrm{d}, J=24.5 \mathrm{~Hz}), 18.6,14.2,14.0,11.3 .{ }^{19} \mathrm{~F}$ NMR $\left(377 \mathrm{MHz}, \mathrm{CDCl}_{3}\right) \delta-147.45$. HRMS (APCI) calcd. for $\mathrm{C}_{23} \mathrm{H}_{42} \mathrm{FO}_{2} \mathrm{Si}[\mathrm{M}+\mathrm{H}]: 397.2933$ found: 397.2962.

Ethyl (2S, 3R)-3-methyl-2-((E)-styryl)-3-(trichloro(oxo)-16-methoxy)heptanoate $(\mathbf{2 x}): \mathrm{R}_{\mathrm{f}}=0.6$ (ether/hexane $=05: 95)$, colourless thick oil, 67\% yield, 95:5 dr determined by 1H-NMR. ${ }^{1} \mathrm{H}$ NMR (400 $\left.\mathrm{MHz}, \mathrm{CDCl}_{3}\right) \delta 7.35-7.30(\mathrm{~m}, 2 \mathrm{H}), 7.29-7.24(\mathrm{~m}, 2 \mathrm{H}), 7.20(\mathrm{dt}, J=7.0,1.7 \mathrm{~Hz}, 1 \mathrm{H}), 6.48(\mathrm{~d}, J=$ $15.8 \mathrm{~Hz}, 1 \mathrm{H}), 6.17$ (dd, $J=15.8,10.0 \mathrm{~Hz}, 1 \mathrm{H}), 4.09$ (dtd, $J=14.4,7.2,3.5 \mathrm{~Hz}, 2 \mathrm{H}), 3.98$ (d, $J=10.0$ $\mathrm{Hz}, 1 \mathrm{H}), 2.19-2.04(\mathrm{~m}, 1 \mathrm{H}), 1.84-1.72(\mathrm{~m}, 1 \mathrm{H}), 1.57(\mathrm{~s}, 3 \mathrm{H}), 1.26-1.15(\mathrm{~m}, 7 \mathrm{H}), 0.86-0.77(\mathrm{~m}$, $3 \mathrm{H}) .{ }^{13} \mathrm{C}$ NMR $\left(101 \mathrm{MHz}, \mathrm{CDCl}_{3}\right) \delta 170.5,155.9(\mathrm{~d}, J=41.5 \mathrm{~Hz}), 136.2,135.7,128.7,128.2,126.6$, 122.4, 90.9, 61.3, 55.9, 35.8, 25.0, 22.7, 20.9, 14.0. HRMS (APCI) calcd. for $\mathrm{C}_{18} \mathrm{H}_{25} \mathrm{O}_{2}[(\mathrm{M}+\mathrm{H})-$ $\left.\mathrm{Cl}_{3} \mathrm{CCO}_{2} \mathrm{H}\right]: 273.1849$ found: 273.1861 .

Ethyl (2S, 3R)-2-((E)-4-bromostyryl)-3-methyl-3-(trichloro(oxo)-16-methoxy)heptanoate (2y): $\mathrm{R}_{\mathrm{f}}=0.4$ (ether/hexane $=05: 95)$, colourless thick oil, 70\% yield, 95:5 dr determined by $1 \mathrm{H}-\mathrm{NMR} .{ }^{1} \mathrm{H}$ NMR $(400$ $\left.\mathrm{MHz}, \mathrm{CDCl}_{3}\right) \delta 7.36(\mathrm{t}, J=9.1 \mathrm{~Hz}, 2 \mathrm{H}), 7.17(\mathrm{~d}, J=8.4 \mathrm{~Hz}, 2 \mathrm{H}), 6.41(\mathrm{~d}, J=15.9 \mathrm{~Hz}, 1 \mathrm{H}), 6.20(\mathrm{dd}, J$ $=15.8,9.9 \mathrm{~Hz}, 1 \mathrm{H}), 4.17-4.03(\mathrm{~m}, 2 \mathrm{H}), 3.99(\mathrm{~d}, J=9.9 \mathrm{~Hz}, 1 \mathrm{H}), 2.09-2.00(\mathrm{~m}, 1 \mathrm{H}), 1.87-1.77(\mathrm{~m}$, $1 \mathrm{H}), 1.62(\mathrm{~s}, 3 \mathrm{H}), 1.36-1.16(\mathrm{~m}, 7 \mathrm{H}), 0.83(\mathrm{t}, J=7.0 \mathrm{~Hz}, 3 \mathrm{H}) .{ }^{13} \mathrm{C} \mathrm{NMR}\left(101 \mathrm{MHz}, \mathrm{CDCl}_{3}\right) \delta 170.5$, $159.9,135.2,134.4,131.8,128.1,123.5,122.0,90.8,61.4,56.0,35.8,25.1,22.8,20.8,14.1,14.0$. HRMS (APCI) calcd. for $\mathrm{C}_{18} \mathrm{H}_{24} \mathrm{BrO}_{2}\left[(\mathrm{M}+\mathrm{H})-\mathrm{Cl}_{3} \mathrm{CCO}_{2} \mathrm{H}\right]: 351.0960$ found: 351.0960 .

Ethyl (2S, 3R)-2-((E)-2-(benzo[d][1,3]dioxol-5-yl)vinyl)-3-methyl-3-(trichloro(oxo)-16methoxy)heptanoate $(\mathbf{2 z}): \mathrm{R}_{\mathrm{f}}=0.4$ (ether/hexane $\left.=05: 95\right)$, colourless thick oil, $67 \%$ yield, $95: 5 \mathrm{dr}$ determined by $1 \mathrm{H}-\mathrm{NMR}$. ${ }^{1} \mathrm{H}$ NMR $\left(400 \mathrm{MHz}, \mathrm{CDCl}_{3}\right) \delta 6.85(\mathrm{~s}, 1 \mathrm{H}), 6.73(\mathrm{~d}, J=8.1 \mathrm{~Hz}, 1 \mathrm{H}), 6.68(\mathrm{~d}$, $J=8.0 \mathrm{~Hz}, 1 \mathrm{H}), 6.38(\mathrm{~d}, J=15.8 \mathrm{~Hz}, 1 \mathrm{H}), 6.02(\mathrm{dd}, J=15.7,9.9 \mathrm{~Hz}, 1 \mathrm{H}), 5.88(\mathrm{~s}, 2 \mathrm{H}), 4.16-4.01$ (m, 2H), $3.95(\mathrm{~d}, J=9.9 \mathrm{~Hz}, 1 \mathrm{H}), 2.08-1.98(\mathrm{~m}, 1 \mathrm{H}), 1.83(\mathrm{dt}, J=15.1,7.1 \mathrm{~Hz}, 1 \mathrm{H}), 1.61(\mathrm{~s}, 3 \mathrm{H}), 1.35-$ $1.14(\mathrm{~m}, 7 \mathrm{H}), 0.83(\mathrm{t}, J=7.0 \mathrm{~Hz}, 3 \mathrm{H}) .{ }^{13} \mathrm{C} \mathrm{NMR}\left(101 \mathrm{MHz}, \mathrm{CDCl}_{3}\right) \delta 170.7,159.9,148.1,147.7,135.1$, 
$130.7,121.5,120.7,108.3,105.8,101.2,91.1,61.3,55.9,35.8,25.1,22.8,20.7,14.1,14.0$. HRMS (APCI) calcd. for $\mathrm{C}_{19} \mathrm{H}_{25} \mathrm{O}_{4}\left[(\mathrm{M}+\mathrm{H})-\mathrm{Cl}_{3} \mathrm{CCO}_{2} \mathrm{H}\right]: 317.17471$ found: 317.1731

Ethyl (2S, 3R)-2-((E)-2,4-difluorostyryl)-3-methyl-3-(trichloro(oxo)-16-methoxy)heptanoate (2aa): $\mathrm{R}_{\mathrm{f}}$ $=0.4($ ether/hexane $=05: 95)$, colourless thick oil, $70 \%$ yield, 95:5 dr determined by $1 \mathrm{H}-\mathrm{NMR} .{ }^{1} \mathrm{H}$ NMR $\left(400 \mathrm{MHz}, \mathrm{CDCl}_{3}\right) \delta 7.35(\mathrm{td}, J=8.6,6.4 \mathrm{~Hz}, 1 \mathrm{H}), 6.82-6.69(\mathrm{~m}, 2 \mathrm{H}), 6.56(\mathrm{~d}, J=16.0 \mathrm{~Hz}, 1 \mathrm{H}), 6.23$ $(\mathrm{dd}, J=16.0,10.0 \mathrm{~Hz}, 1 \mathrm{H}), 4.17-4.09(\mathrm{~m}, 1 \mathrm{H}), 4.09-4.02(\mathrm{~m}, 1 \mathrm{H}), 3.98(\mathrm{~d}, J=10.0 \mathrm{~Hz}, 1 \mathrm{H}), 2.10-$ $2.01(\mathrm{~m}, 1 \mathrm{H}), 1.84(\mathrm{dt}, J=21.6,7.2 \mathrm{~Hz}, 1 \mathrm{H}), 1.62(\mathrm{~s}, 3 \mathrm{H}), 1.36-1.26(\mathrm{~m}, 3 \mathrm{H}), 1.22(\mathrm{t}, J=7.1 \mathrm{~Hz}, 4 \mathrm{H})$, $0.84(\mathrm{t}, J=7.1 \mathrm{~Hz}, 3 \mathrm{H}) .{ }^{13} \mathrm{C} \mathrm{NMR}\left(101 \mathrm{MHz}, \mathrm{CDCl}_{3}\right) \delta 170.5,159.9,128.5,127.1,125.0,120.5,111.5$, 104.2, 90.8, 61.4, 56.4, 35.8, 30.4, 25.1, 22.7, 20.8, 14.1, 13.9. ${ }^{19} \mathrm{~F}$ NMR $\left(377 \mathrm{MHz}, \mathrm{CDCl}_{3}\right) \delta-109.95$, -109.97, -113.41, -113.43. HRMS (APCI) calcd. for $\mathrm{C}_{18} \mathrm{H}_{23} \mathrm{~F}_{2} \mathrm{O}_{2}\left[(\mathrm{M}+\mathrm{H})-\mathrm{Cl}_{3} \mathrm{CCO}_{2} \mathrm{H}\right]: 309.1661$ found: 309.1679 .

Ethyl (2S, 3R)-2-((E)-2-(furan-3-yl)vinyl)-3-methyl-3-(trichloro(oxo)-16-methoxy)heptanoate (2ab): The reaction was performed at $0{ }^{\circ} \mathrm{C}$, in order to reduce the decomposition and byproducts. $\mathrm{R}_{\mathrm{f}}=0.6$ (ether/hexane $=05: 95$ ), colourless thick oil, $40 \%$ yield, 95:5 dr determined by 1H-NMR. ${ }^{1} \mathrm{H}$ NMR $\left(400 \mathrm{MHz}, \mathrm{CDCl}_{3}\right) \delta 7.35(\mathrm{~d}, J=1.7 \mathrm{~Hz}, 1 \mathrm{H}), 6.40-6.34(\mathrm{~m}, 1 \mathrm{H}), 6.27(\mathrm{~d}, J=3.3 \mathrm{~Hz}, 1 \mathrm{H}), 6.18(\mathrm{dd}$, $J=15.7,10.0 \mathrm{~Hz}, 1 \mathrm{H}), 4.22-4.17(\mathrm{~m}, 1 \mathrm{H}), 4.14-4.08(\mathrm{~m}, 1 \mathrm{H}), 4.01(\mathrm{~d}, J=10.0 \mathrm{~Hz}, 1 \mathrm{H}), 2.11(\mathrm{dd}, J$ $=15.4,6.8 \mathrm{~Hz}, 1 \mathrm{H}), 1.91(\mathrm{dt}, J=15.1,7.4 \mathrm{~Hz}, 1 \mathrm{H}), 1.69(\mathrm{~s}, 3 \mathrm{H}), 1.35-1.23(\mathrm{~m}, 7 \mathrm{H}), 0.90(\mathrm{t}, J=7.0$ $\mathrm{Hz}, 3 \mathrm{H}) .{ }^{13} \mathrm{C}$ NMR $\left(101 \mathrm{MHz}, \mathrm{CDCl}_{3}\right) \delta 170.5,159.9,151.8,142.4,123.6,120.9,111.4,108.9,91.1$, $61.3,55.7,35.8,31.5,30.2,29.7,25.02,22.7,20.6,14.1,14.0$. HRMS (APCI) calcd. for $\mathrm{C}_{18} \mathrm{H}_{24} \mathrm{BrO}_{2}$ [(M+H)- $\left.\mathrm{Cl}_{3} \mathrm{CCO}_{2} \mathrm{H}\right]: 263.1642$ found: 263.1661 .

Ethyl (2S, 3R)-3-methyl-2-((E)-prop-1-en-1-yl)-3-(2,2,2-trichloroacetoxy)heptanoate (2ac): $\mathrm{R}_{\mathrm{f}}=0.6$ (ether/hexane $=05: 95)$, colourless thick oil, 33\% yield along with $20 \%$ of eliminated product, $95: 5 \mathrm{dr}$ determined by $1 \mathrm{H}-\mathrm{NMR} .{ }^{1} \mathrm{H}$ NMR $\left(400 \mathrm{MHz}, \mathrm{CDCl}_{3}\right) \delta 6.26(\mathrm{dt}, J=16.8,10.3 \mathrm{~Hz}, 1 \mathrm{H}), 6.12(\mathrm{dd}, J=$ $15.1,10.5 \mathrm{~Hz}, 1 \mathrm{H}), 5.68(\mathrm{dd}, J=15.1,10.0 \mathrm{~Hz}, 1 \mathrm{H}), 5.17(\mathrm{~d}, J=16.5 \mathrm{~Hz}, 1 \mathrm{H}), 5.07(\mathrm{~d}, J=10.5 \mathrm{~Hz}$, $1 \mathrm{H}), 4.17-3.99(\mathrm{~m}, 2 \mathrm{H}), 3.86(\mathrm{~d}, J=10.0 \mathrm{~Hz}, 1 \mathrm{H}), 2.04-1.90(\mathrm{~m}, 1 \mathrm{H}), 1.86-1.73(\mathrm{~m}, 1 \mathrm{H}), 1.57(\mathrm{~s}$, $3 \mathrm{H}), 1.32-1.25(\mathrm{~m}, 4 \mathrm{H}), 1.22-1.15(\mathrm{~m}, 4 \mathrm{H}), 0.87-0.76(\mathrm{~m}, 3 \mathrm{H}) .{ }^{13} \mathrm{C}$ NMR $(101 \mathrm{MHz}, \mathrm{CDCl} 3) \delta$ $170.6,159.8,136.3,126.4,118.6,91.0,61.2,55.6,35.7,25.0,22.7,22.6,20.6,14.1,14.0$. HRMS (APCI) calcd. for $\mathrm{C}_{14} \mathrm{H}_{23} \mathrm{O}_{2}\left[(\mathrm{M}+\mathrm{H})-\mathrm{Cl}_{3} \mathrm{CCO}_{2} \mathrm{H}\right]: 223.1693$ found: 223.1701 .

(4S,5R)-4-((E)-4-methoxystyryl)-5-methylnon-1-en-5-yl 2,2,2-trichloroacetate $\quad(\mathbf{2 a d}): \quad \mathrm{R}_{\mathrm{f}}=0.7$ (ether/hexane $=05: 95)$, colourless thick oil, 85\% yield, 98:2 dr determined by $1 \mathrm{H}-\mathrm{NMR}$, crude product itself clean, no further purification required. ${ }^{1} \mathrm{H} \mathrm{NMR}\left(400 \mathrm{MHz}, \mathrm{CDCl}_{3}\right) \delta 7.20(\mathrm{~d}, J=8.7 \mathrm{~Hz}, 2 \mathrm{H})$, $6.77(\mathrm{~d}, J=8.7 \mathrm{~Hz}, 2 \mathrm{H}), 6.29(\mathrm{~d}, J=15.8 \mathrm{~Hz}, 1 \mathrm{H}), 5.78-5.69(\mathrm{~m}, 1 \mathrm{H}), 5.69-5.59(\mathrm{~m}, 1 \mathrm{H}), 4.97-$ $4.87(\mathrm{~m}, 2 \mathrm{H}), 3.71(\mathrm{~s}, 3 \mathrm{H}), 2.83(\mathrm{td}, J=10.7,2.7 \mathrm{~Hz}, 1 \mathrm{H}), 2.45-2.35(\mathrm{~m}, 1 \mathrm{H}), 2.15-2.06(\mathrm{~m}, 1 \mathrm{H})$, $2.04-1.94(\mathrm{~m}, 1 \mathrm{H}), 1.83(\mathrm{ddd}, J=14.4,11.4,4.7 \mathrm{~Hz}, 1 \mathrm{H}), 1.48(\mathrm{~s}, 3 \mathrm{H}), 1.31-1.19(\mathrm{~m}, 4 \mathrm{H}), 0.82(\mathrm{t}, J$ $=7.0 \mathrm{~Hz}, 3 \mathrm{H}) .{ }^{13} \mathrm{C}$ NMR $\left(101 \mathrm{MHz}, \mathrm{CDCl}_{3}\right) \delta 159.9,159.2,136.7,133.2,129.9,127.4,125.7,116.3$, 114.0, 113.9, 93.3, 55.4, 50.3, 35.8, 33.8, 25.2, 22.9, 20.7, 14.1. HRMS (APCI) calcd. for $\mathrm{C}_{19} \mathrm{H}_{27} \mathrm{O}$ $\left[(\mathrm{M}+\mathrm{H})-\mathrm{Cl}_{3} \mathrm{CCO}_{2} \mathrm{H}\right]: 271.2056$ found: 271.2081 .

(R, E)-1-(4-methoxyphenyl)-4-methyloct-1-en-4-yl 2,2,2-trichloroacetate (2ae): $\mathrm{R}_{\mathrm{f}}=0.7$ (ether/hexane $=05: 95)$, colourless thick oil, $90 \%$ yield, $98: 2 \mathrm{dr}$ determined by $1 \mathrm{H}-\mathrm{NMR}$, crude product itself clean, no further purification required. ${ }^{1} \mathrm{H}$ NMR $\left(400 \mathrm{MHz}, \mathrm{CDCl}_{3}\right) \delta 7.19(\mathrm{~d}, J=8.7 \mathrm{~Hz}, 2 \mathrm{H}), 6.76(\mathrm{~d}, J=8.7$ $\mathrm{Hz}, 2 \mathrm{H}), 6.34$ (d, $J=15.8 \mathrm{~Hz}, 1 \mathrm{H}), 5.99-5.89$ (m, 1H), 3.72 (s, 3H), 2.75 (ddd, $J=14.1,7.3,0.7 \mathrm{~Hz}$, $1 \mathrm{H}), 2.63$ (ddd, $J=14.3,7.6,0.8 \mathrm{~Hz}, 1 \mathrm{H}), 1.97-1.86(\mathrm{~m}, 1 \mathrm{H}), 1.76$ (dt, $J=14.3,7.2 \mathrm{~Hz}, 1 \mathrm{H}), 1.49$ (s, $3 \mathrm{H}), 1.33-1.23(\mathrm{~m}, 4 \mathrm{H}), 0.84(\mathrm{t}, J=7.1 \mathrm{~Hz}, 3 \mathrm{H}) .{ }^{13} \mathrm{C} \mathrm{NMR}\left(101 \mathrm{MHz}, \mathrm{CDCl}_{3}\right) \delta 160.2,159.1,133.7$, 130.1, 127.3, 127.3, 121.3, 114.0, 91.2, 55.3, 41.6, 37.8, 25.6, 23.2, 22.9, 14.1. HRMS (APCI) calcd. for $\mathrm{C}_{16} \mathrm{H}_{23} \mathrm{O}\left[(\mathrm{M}+\mathrm{H})-\mathrm{Cl}_{3} \mathrm{CCO}_{2} \mathrm{H}\right]: 231.1743$ found: 231.1764 .

(4S, 5R)-5-methyl-4-(2-methylprop-1-en-1-yl)-2-phenylnon-1-en-5-yl 2,2,2-trichloroacetate (2af): $\mathrm{R}_{\mathrm{f}}$ $=0.5$ (ether/hexane $=05: 95)$, colourless thick oil, $75 \%$ yield along with a $13 \%$ of eliminated product, 95:5 dr determined by $1 \mathrm{H}$ NMR. ${ }^{1} \mathrm{H}$ NMR $\left(400 \mathrm{MHz}, \mathrm{CDCl}_{3}\right) \delta 7.30(\mathrm{~d}, J=7.2 \mathrm{~Hz}, 2 \mathrm{H}), 7.23(\mathrm{~d}, J=$ $7.0 \mathrm{~Hz}, 2 \mathrm{H}), 7.19-7.15(\mathrm{~m}, 1 \mathrm{H}), 5.16(\mathrm{~d}, J=1.2 \mathrm{~Hz}, 1 \mathrm{H}), 4.89(\mathrm{~s}, 1 \mathrm{H}), 4.73(\mathrm{~d}, J=10.5 \mathrm{~Hz}, 1 \mathrm{H}), 2.97$ (dd, $J=17.9,8.0 \mathrm{~Hz}, 2 \mathrm{H}), 2.22(\mathrm{dd}, J=13.0,11.0 \mathrm{~Hz}, 1 \mathrm{H}), 1.89-1.81(\mathrm{~m}, 2 \mathrm{H}), 1.54(\mathrm{~s}, 3 \mathrm{H}), 1.45(\mathrm{~s}$, $3 \mathrm{H}), 1.20-1.12(\mathrm{~m}, 4 \mathrm{H}), 1.10(\mathrm{~s}, 3 \mathrm{H}), 0.76(\mathrm{t}, J=6.9 \mathrm{~Hz}, 3 \mathrm{H}) .{ }^{13} \mathrm{C} \mathrm{NMR}\left(101 \mathrm{MHz}, \mathrm{CDCl}_{3}\right) \delta 158.8$, 
145.5, 139.6, 134.5, 127.1, 127.1, 126.3, 125.4, 121.6, 113.4, 93.4, 42.3, 34.8, 34.7, 24.9, 24.0, 21.8, 18.9, 17.4, 12.9. HRMS (APCI) calcd. for $\mathrm{C}_{20} \mathrm{H}_{29}\left[(\mathrm{M}+\mathrm{H})-\mathrm{Cl}_{3} \mathrm{CCO}_{2} \mathrm{H}\right]: 269.2264$ found: 269.2280 .

(3S, 4R, E)-1-(4-methoxyphenyl)-3,4-dimethyloct-1-en-4-yl 2,2,2-trichloroacetate (2ag): Rf $=0.5$ (ether/hexane $=03: 97$ ), colourless thick oil, 84\% yield, single isomer determined by ${ }^{1} \mathrm{H}-\mathrm{NMR} .{ }^{1} \mathrm{H}$ NMR $\left(400 \mathrm{MHz}, \mathrm{CDCl}_{3}\right) \delta 7.23-7.19(\mathrm{~m}, 2 \mathrm{H}), 6.80-6.74(\mathrm{~m}, 2 \mathrm{H}), 6.33(\mathrm{~d}, J=15.8 \mathrm{~Hz}, 1 \mathrm{H}), 5.89$ (dd, $J=$ $15.8,8.9 \mathrm{~Hz}, 1 \mathrm{H}), 3.73$ (s, 3H), 3.01 (dq, $J=13.9,7.0 \mathrm{~Hz}, 1 \mathrm{H}), 2.05-1.96(\mathrm{~m}, 1 \mathrm{H}), 1.81$ (ddd, $J=$ $14.3,11.4,5.0 \mathrm{~Hz}, 1 \mathrm{H}), 1.46(\mathrm{~s}, 3 \mathrm{H}), 1.26(\mathrm{ddt}, J=13.1,9.3,6.5 \mathrm{~Hz}, 4 \mathrm{H}), 1.10(\mathrm{~d}, J=6.9 \mathrm{~Hz}, 3 \mathrm{H}), 0.83$ $(\mathrm{t}, J=7.1 \mathrm{~Hz}, 3 \mathrm{H}) .{ }^{13} \mathrm{C} \mathrm{NMR}\left(101 \mathrm{MHz}, \mathrm{CDCl}_{3}\right) \delta 160.0,159.1,131.2,130.1,127.9,127.2,114.0,93.6$, 91.2, 55.4, 44.0, 35.5, 25.4, 22.9, 20.4, 15.3, 14.1. HRMS (APCI) calcd. for $\mathrm{C}_{18} \mathrm{H}_{24} \mathrm{BrO}_{2}$ [M-OCOCCl 3 : 351.0960 found: 351.0960 .

((3S, 4S, E)-4-bromo-3-ethylpent-1-ene-1,4-diyl)dibenzene (2ah): $\mathrm{R}_{\mathrm{f}}=0.6$ (ether/hexane $=05: 95$ ), colourless thick oil, $60 \%$ yield, 1:1 dr crude NMR, 1:0.63 dr after column purification. ${ }^{1} \mathrm{H}$ NMR (400 $\mathrm{MHz}, \mathrm{CDCl} 3) \delta 7.43-7.12(\mathrm{~m}, 19 \mathrm{H}), 6.42(\mathrm{~d}, \mathrm{~J}=15.9 \mathrm{~Hz}, 0.64 \mathrm{H}), 6.36(\mathrm{~d}, \mathrm{~J}=15.9 \mathrm{~Hz}, 1 \mathrm{H}), 5.96(\mathrm{dd}$, $\mathrm{J}=15.9,9.6 \mathrm{~Hz}, 1 \mathrm{H}), 5.86(\mathrm{dd}, \mathrm{J}=15.9,9.7 \mathrm{~Hz}, 0.62 \mathrm{H}), 2.30-2.22(\mathrm{~m}, 1.7 \mathrm{H}), 1.53(\mathrm{~s}, 2 \mathrm{H}), 1.47(\mathrm{~s}$, $3 \mathrm{H}), 1.39-1.31(\mathrm{~m}, 1.72 \mathrm{H}), 1.16(\mathrm{ddd}, \mathrm{J}=13.4,11.2,7.3 \mathrm{~Hz}, 1.8 \mathrm{H}), 0.72-0.68(\mathrm{~m}, 5.2 \mathrm{H}) .{ }^{13} \mathrm{C}$ NMR $(101 \mathrm{MHz}, \mathrm{CDCl} 3) \delta 147.4,146.4,137.4,137.3,134.0,133.4,130.4,130.2,128.6,128.6,128.0,127.9$ ,127.8, 127.4, 126.8, 126.5, 126.3, 126.2, 126.0, 125.2, 58.1, 57.0, 31.2, 30.7, 29.3, 26.0, 22.3, 22.1, 12.7, 12.7. HRMS (APCI) calcd. for $\mathrm{C}_{19} \mathrm{H}_{22} \mathrm{Br}[\mathrm{M}+\mathrm{H}]: 329.0905$ found: 329.0913 .

Ethyl (2S, 3S)-3-hydroxy-3-methyl-4-oxo-2-((E)-styryl)nonanoate (5b): ${ }^{1} \mathrm{H}$ NMR (400 $\mathrm{MHz}, \mathrm{CDCl}_{3}$ ) $\delta 7.36-7.30(\mathrm{~m}, 2 \mathrm{H}), 7.25(\mathrm{dd}, J=8.2,6.7 \mathrm{~Hz}, 2 \mathrm{H}), 7.17(\mathrm{~s}, 1 \mathrm{H}), 6.45(\mathrm{~d}, J=15.9 \mathrm{~Hz}, 1 \mathrm{H}), 6.29(\mathrm{dd}$, $J=15.9,9.6 \mathrm{~Hz}, 1 \mathrm{H}), 4.13(\mathrm{tdd}, J=10.8,7.1,3.7 \mathrm{~Hz}, 2 \mathrm{H}), 3.14(\mathrm{~d}, J=9.6 \mathrm{~Hz}, 1 \mathrm{H}), 1.46-1.39(\mathrm{~m}$, 2H), $1.32(\mathrm{dd}, J=6.1,3.0 \mathrm{~Hz}, 2 \mathrm{H}), 1.21(\mathrm{dd}, J=9.6,4.6 \mathrm{~Hz}, 10 \mathrm{H}), 1.09(\mathrm{~s}, 3 \mathrm{H}), 0.81(\mathrm{t}, J=6.8 \mathrm{~Hz}$, $3 \mathrm{H}) .{ }^{13} \mathrm{C}$ NMR $\left(101 \mathrm{MHz}, \mathrm{CDCl}_{3}\right) \delta 174.1,136.7,134.6,128.6,127.8,126.5,124.2,73.9,61.1,58.2$, 41.8, 31.8, 29.8, 23.8, 23.7, 22.6, 14.2, 14.1. HRMS (APCI) calcd. for $\mathrm{C}_{20} \mathrm{H}_{27} \mathrm{O}_{3}\left[(\mathrm{M}+\mathrm{H})-\mathrm{Cl}_{3} \mathrm{CCO}_{2} \mathrm{H}\right]$ : 315.1955 found: 315.1941 .

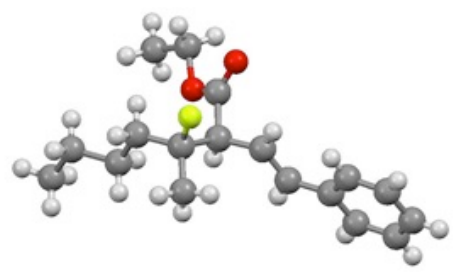<smiles>CCOC(=O)C(/C=C/c1ccccc1)C(C)(C)Br</smiles>

1. Crystal structure for 2s. (CCDC no: 1977388)

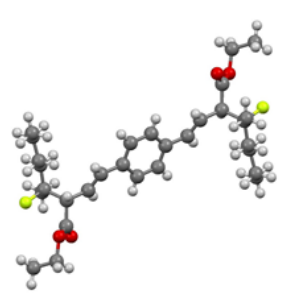<smiles>CCOC(=O)C(/C=C/c1ccc(/C=C/C(C(=O)OCC)C(C)(C)F)cc1)C(C)(C)C</smiles> 


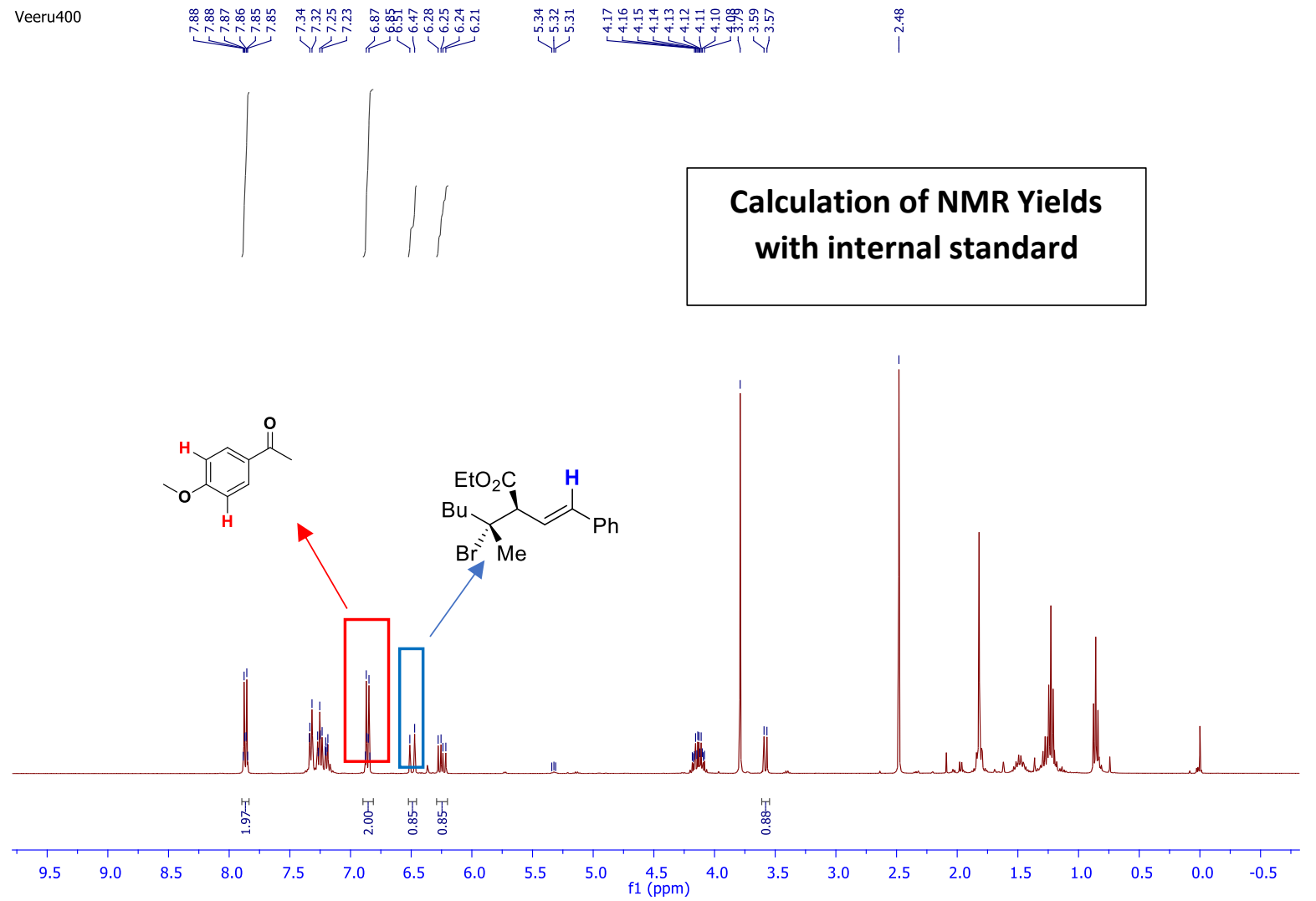

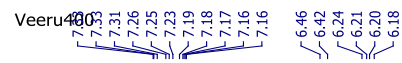

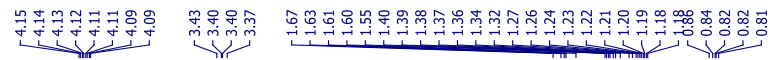
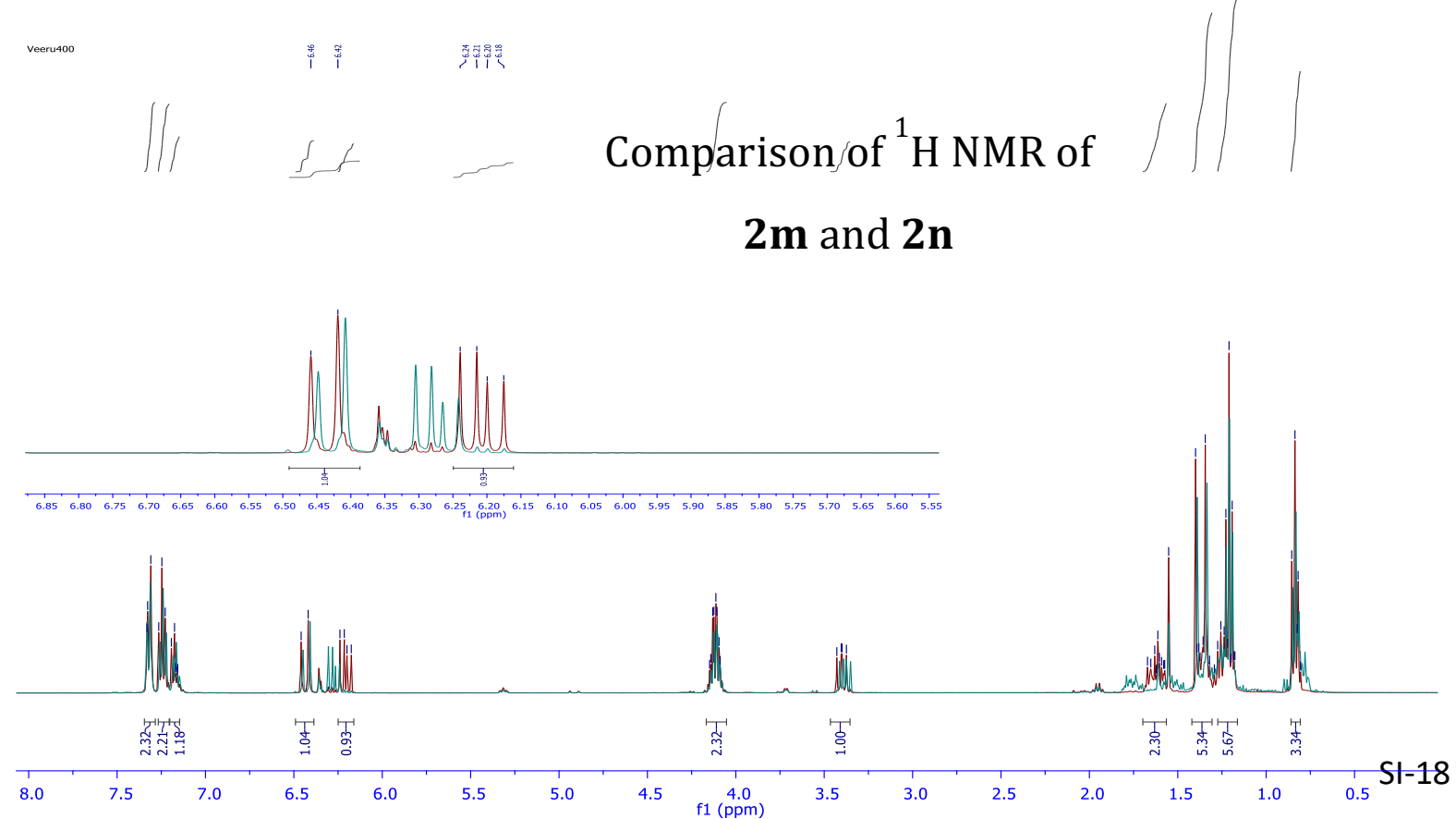


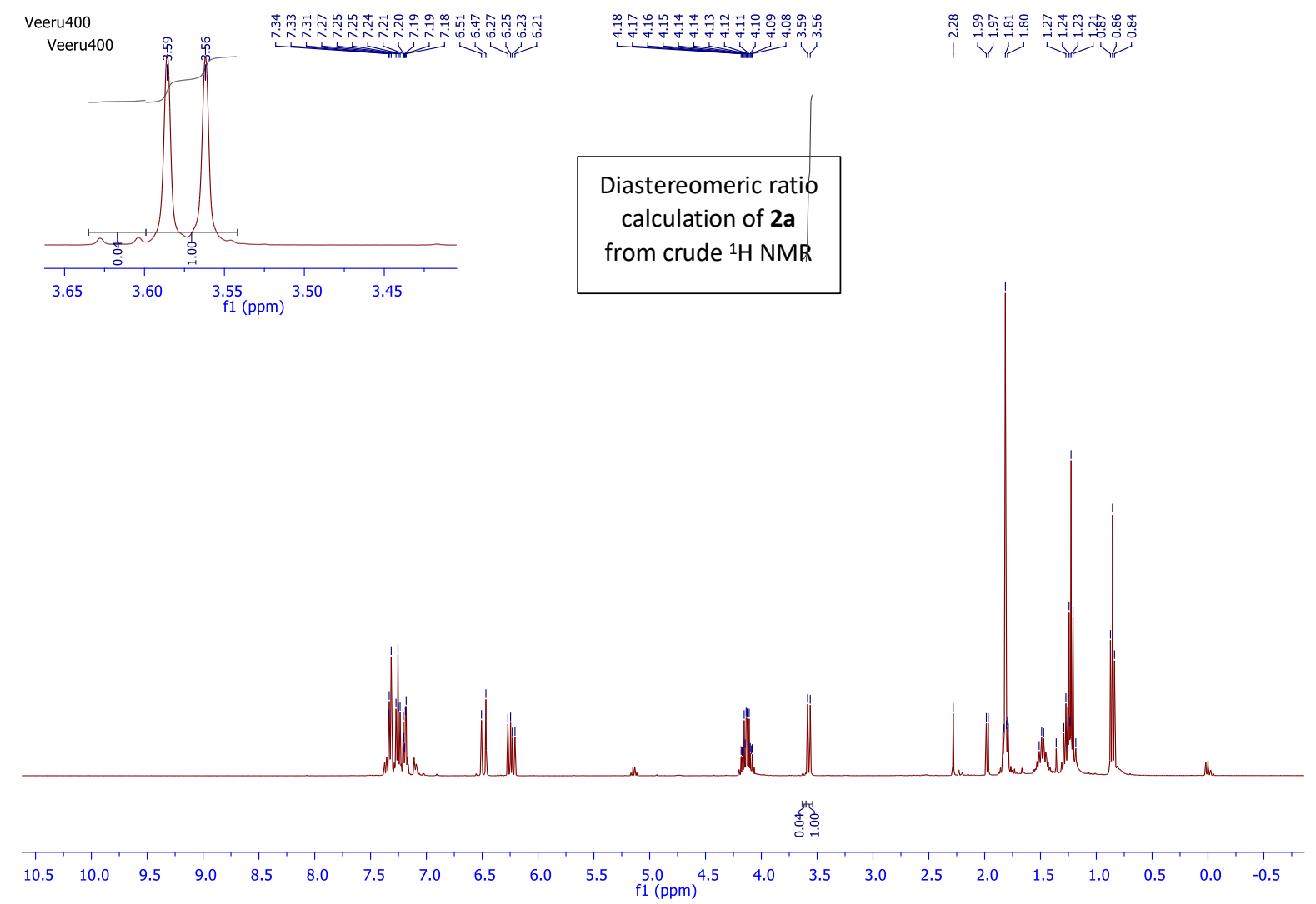

2. HPLC data for compound $\mathbf{2 q}$
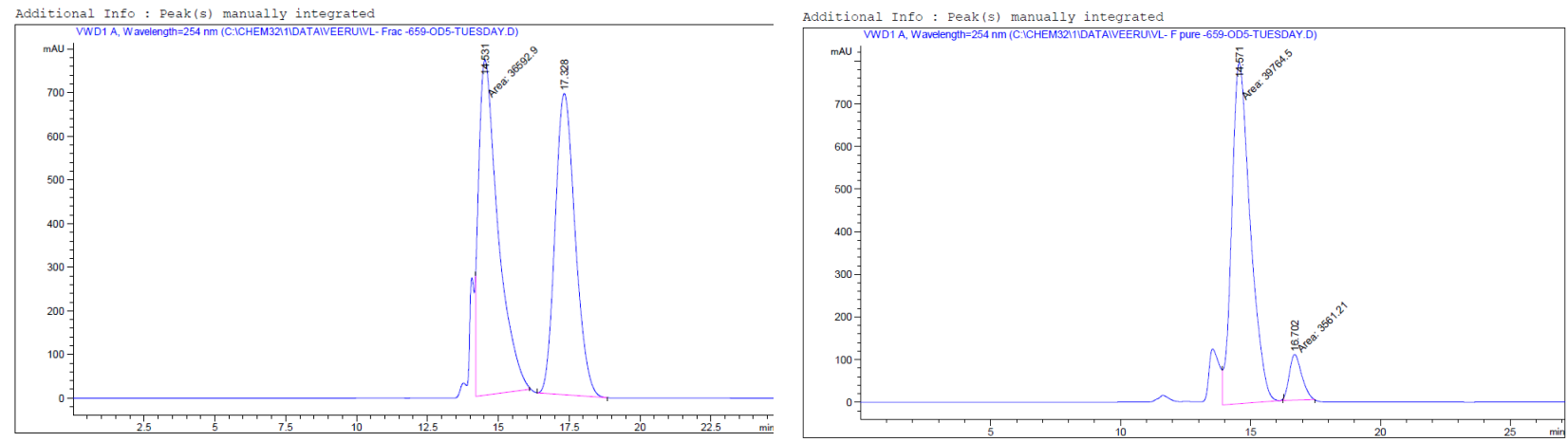

Area Percent Report 


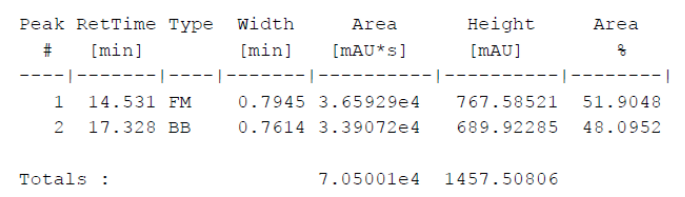

*** End of Report ***

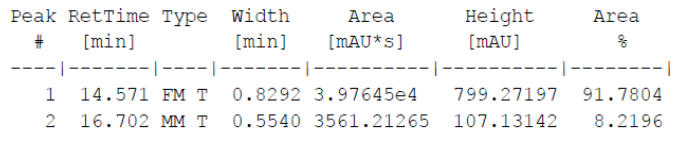

Totals: $\quad 4.33257 e 4 \quad 906.40340$

\section{HPLC data for compound $\mathbf{5 b}$}

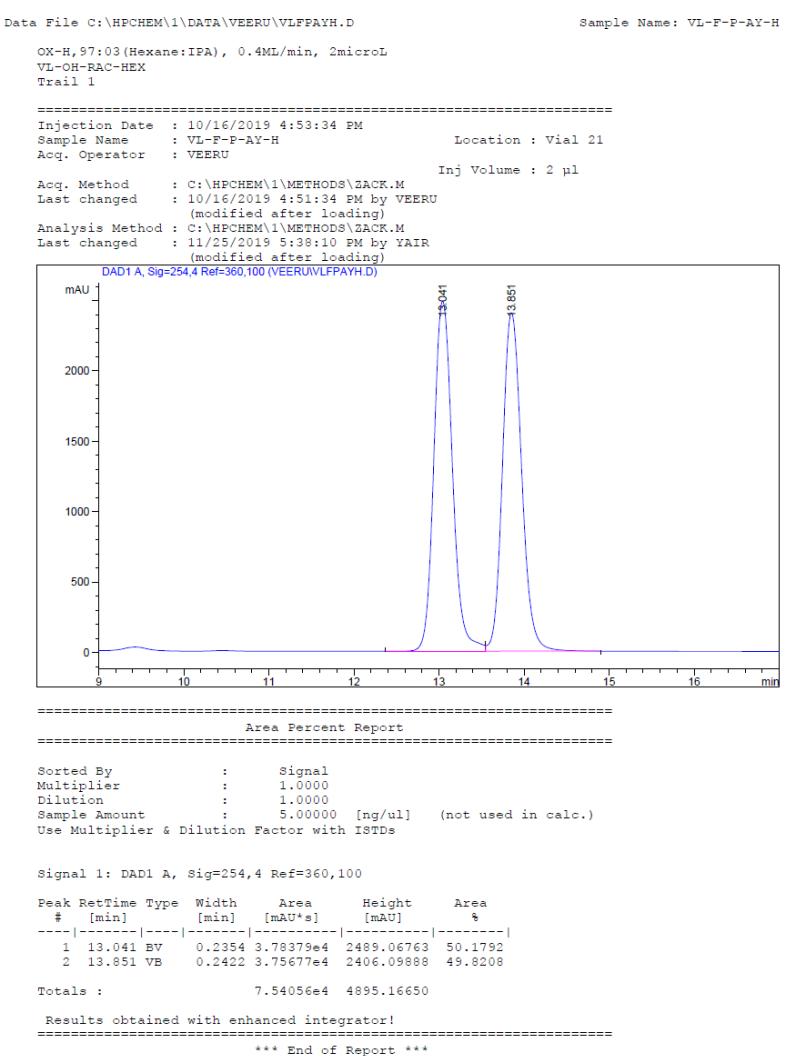

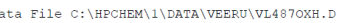
OX-H, 97:3(Hexane:IPA), $0.4 \mathrm{ML} / \mathrm{min}, 2 \mathrm{microL}$

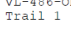

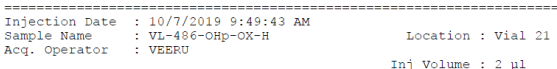

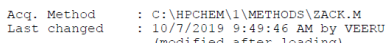
analysis Method : C: $\backslash$ HPCHEM $\backslash 1 \backslash$ METTHODS $\backslash Z A C R . M$ (modified after loading)

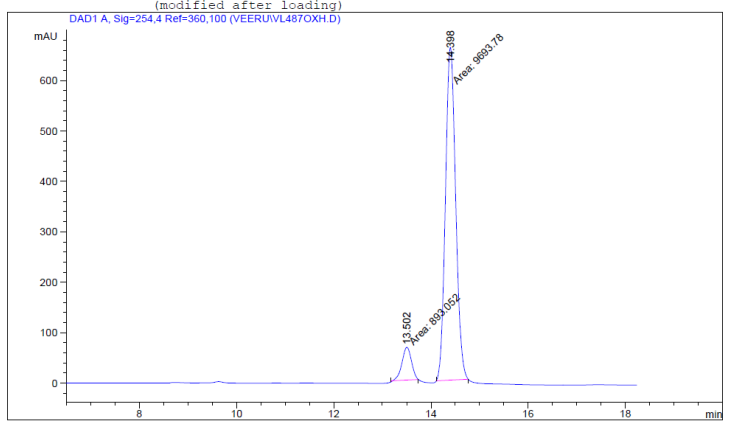

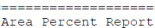

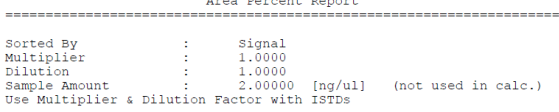

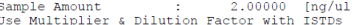

1: DAD1 A, Sig=254, 4 Ref $=360,100$

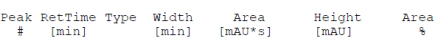

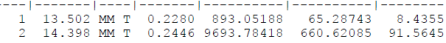

$1.05868 \mathrm{e} 4 \quad 725.90628$

Results obtained with enhanced integrator:

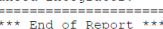

\section{Spectral Data}


<smiles>CCOC(=O)C1C(C(=O)OCC)C1[13C](=O)CC</smiles>

$78 \%, 1: 1 \mathrm{dr}$
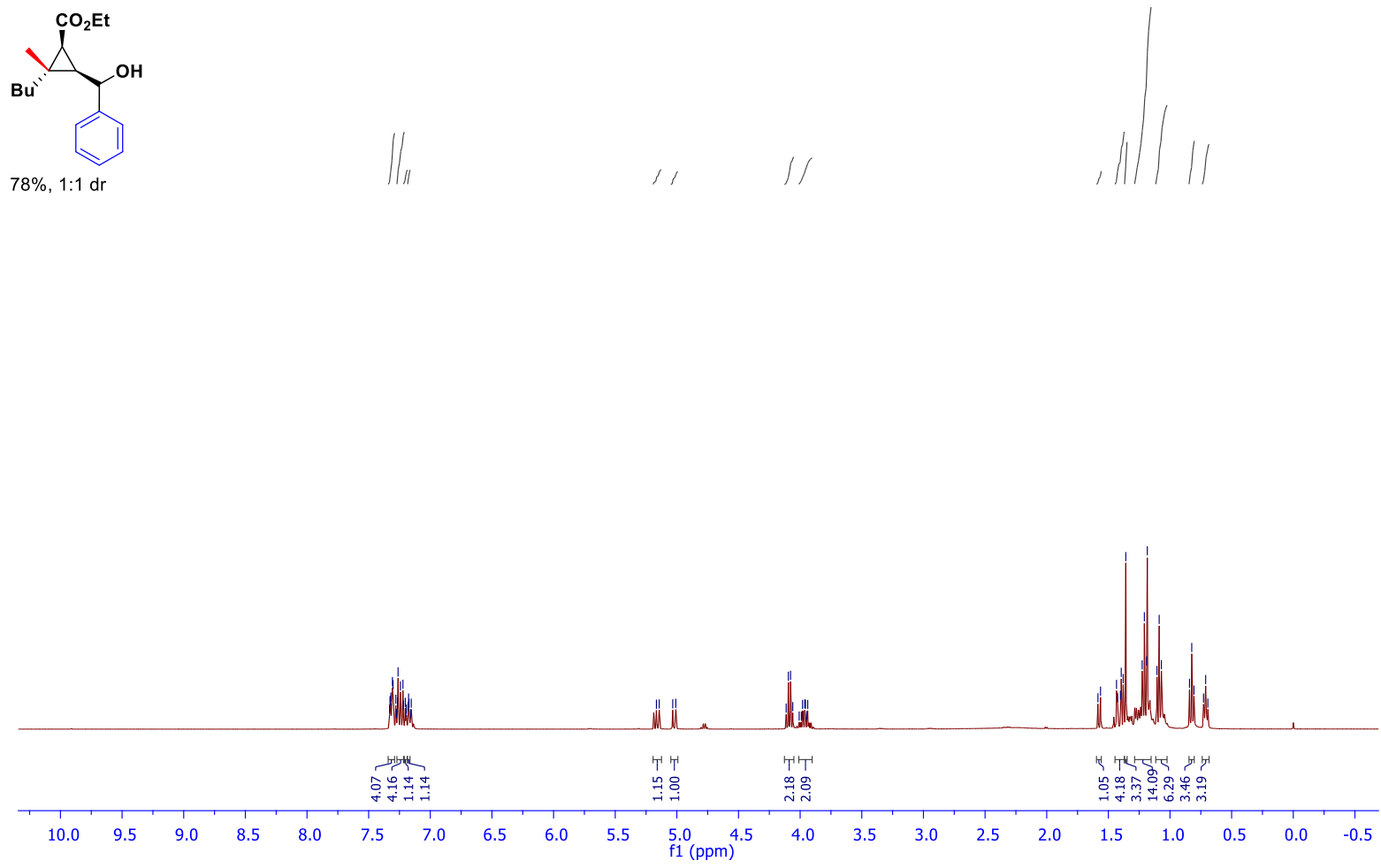

Veeru4002019

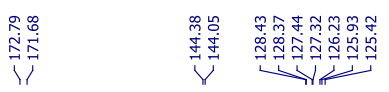

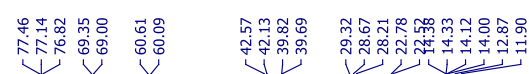

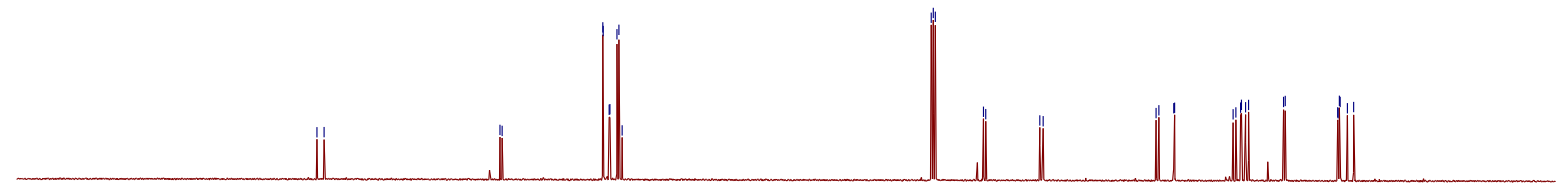

$\begin{array}{lllllllllllllllllllllllll}210 & 200 & 190 & 180 & 170 & 160 & 150 & 140 & 130 & 120 & 110 & 100 & 100 & 80 & 70 & 60 & 50 & 40 & 30 & 20 & 10 & 0 & -10\end{array}$ 


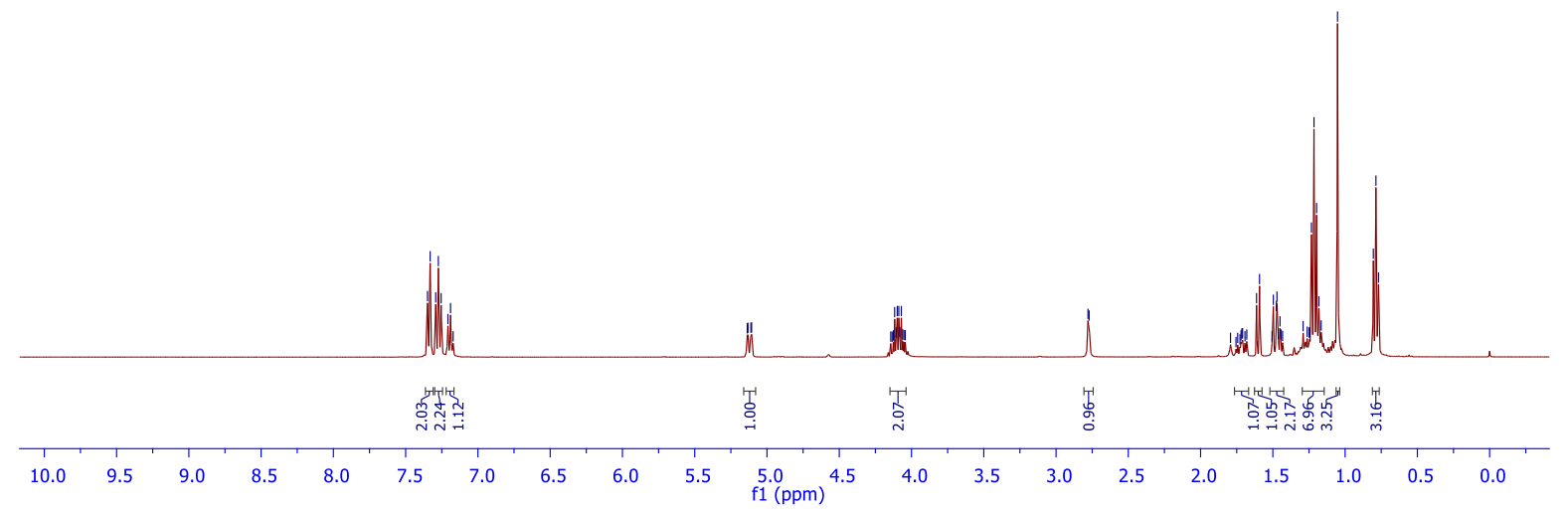

Veeru400

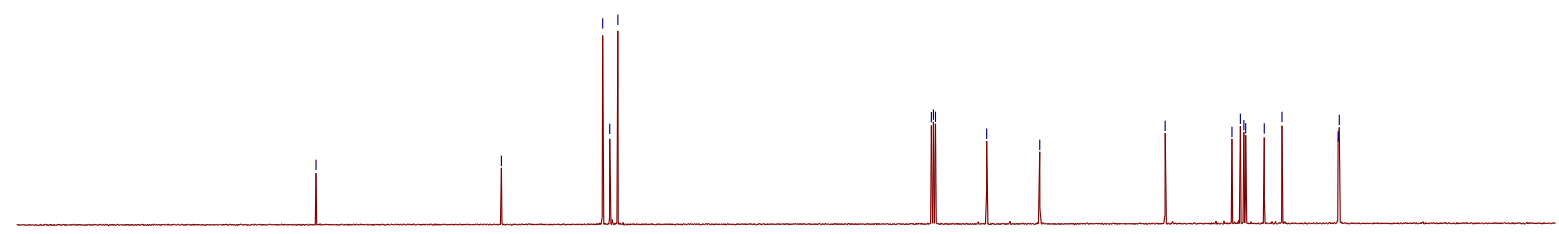

$\begin{array}{llllllllllllllllllllllllll}210 & 200 & 190 & 180 & 170 & 160 & 150 & 140 & 130 & 120 & 110 & 100 & 100 & 80 & 70 & 60 & 50 & 40 & 30 & 20 & 10 & 0 & -10\end{array}$ 
<smiles>CCOC(O)C(C)(C)C(C)(C)C</smiles>
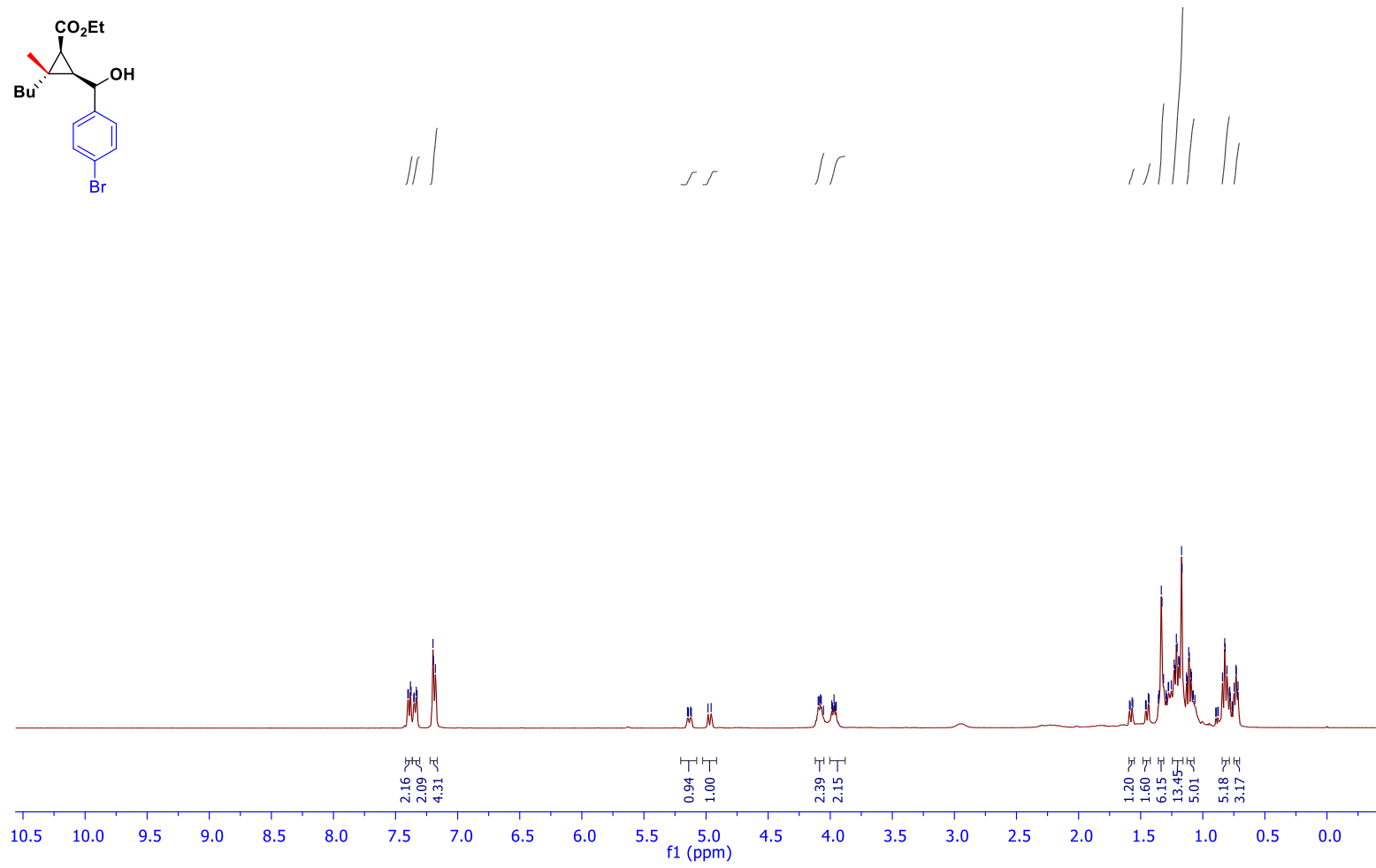

Veeru4002019

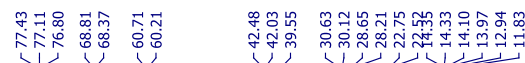

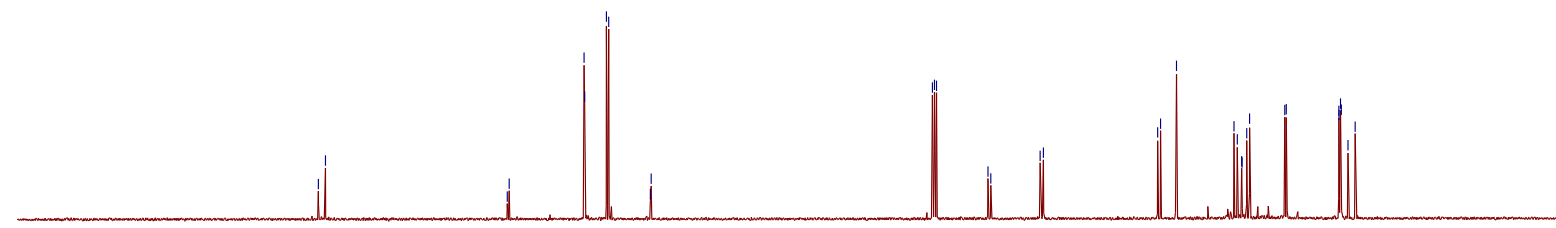

$\begin{array}{llllllllllllllllllllllllllll}210 & 200 & 190 & 180 & 170 & 160 & 150 & 140 & 130 & 120 & 110 & 100 & 100 & 80 & 70 & 60 & 50 & 40 & 30 & 20 & 10 & 0 & -10\end{array}$ 


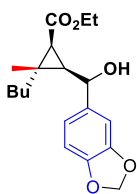

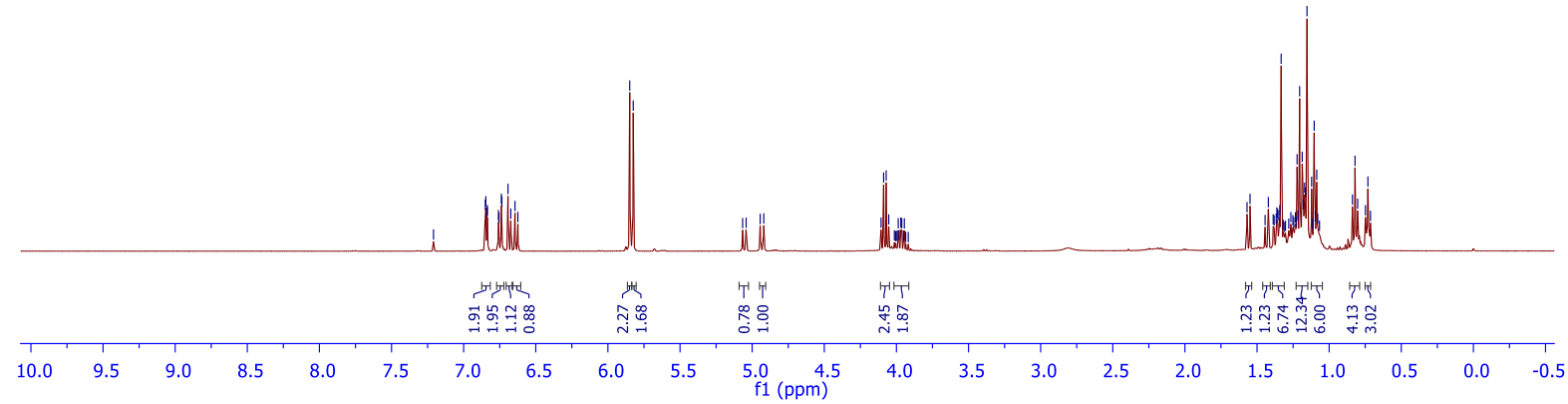

Veeru4002019

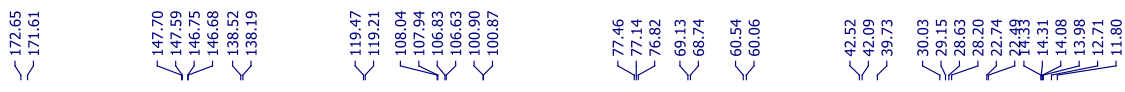

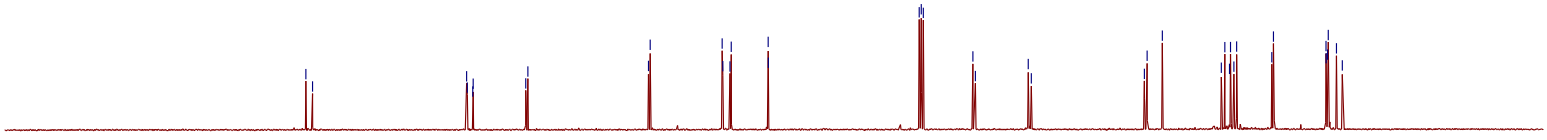

$\begin{array}{lllllllllllllllllllllllllll}210 & 200 & 190 & 180 & 170 & 160 & 150 & 140 & 130 & 120 & 110 & \begin{array}{c}100 \\ \mathrm{f} 1(\mathrm{ppm})\end{array} & 90 & 80 & 70 & 60 & 50 & 40 & 30 & 20 & 10 & 0 & -10\end{array}$ 


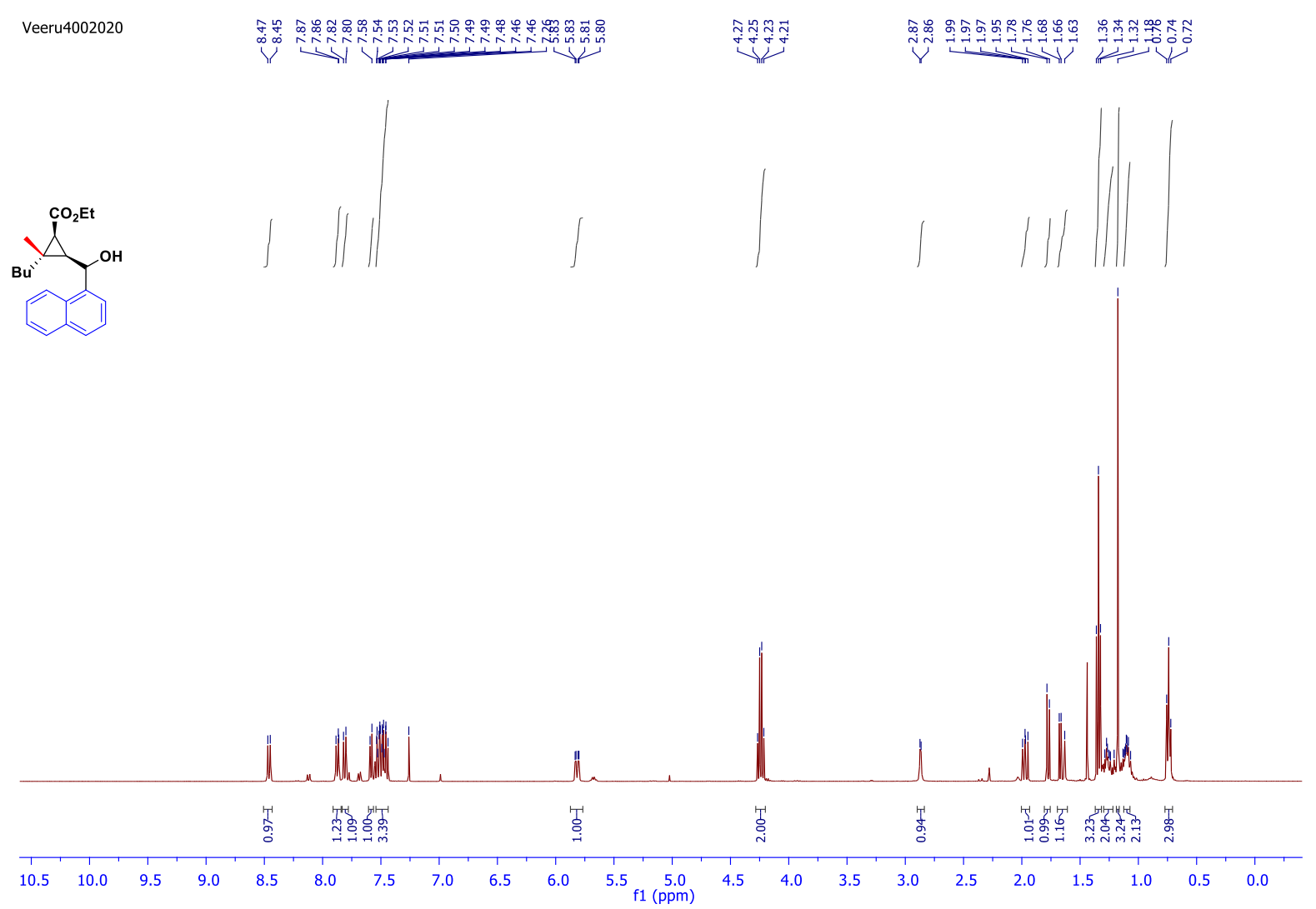

Veeru4002020

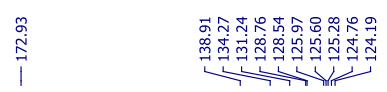

诲

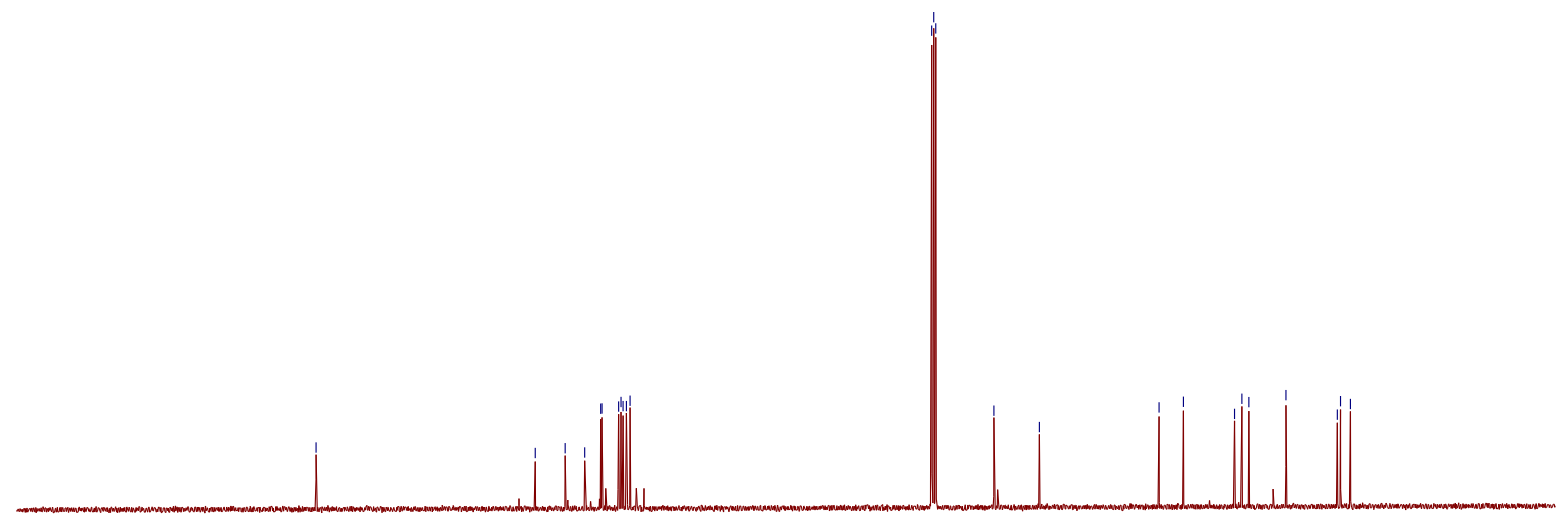

$\begin{array}{llllllllllllllllllllllll}210 & 200 & 190 & 180 & 170 & 160 & 150 & 140 & 130 & 120 & 110 & \begin{array}{c}100 \\ \mathrm{f} 1(\mathrm{ppm})\end{array} & 90 & 80 & 70 & 60 & 50 & 40 & 30 & 20 & 10 & 0 & -10\end{array}$ 
Veeru400

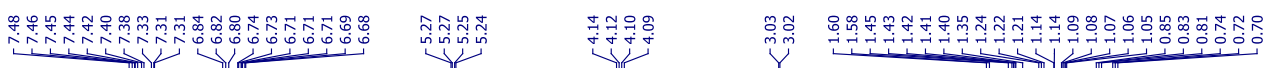<smiles>CCOC(O)C(c1ccc(F)cc1F)C(C)(C)C</smiles>
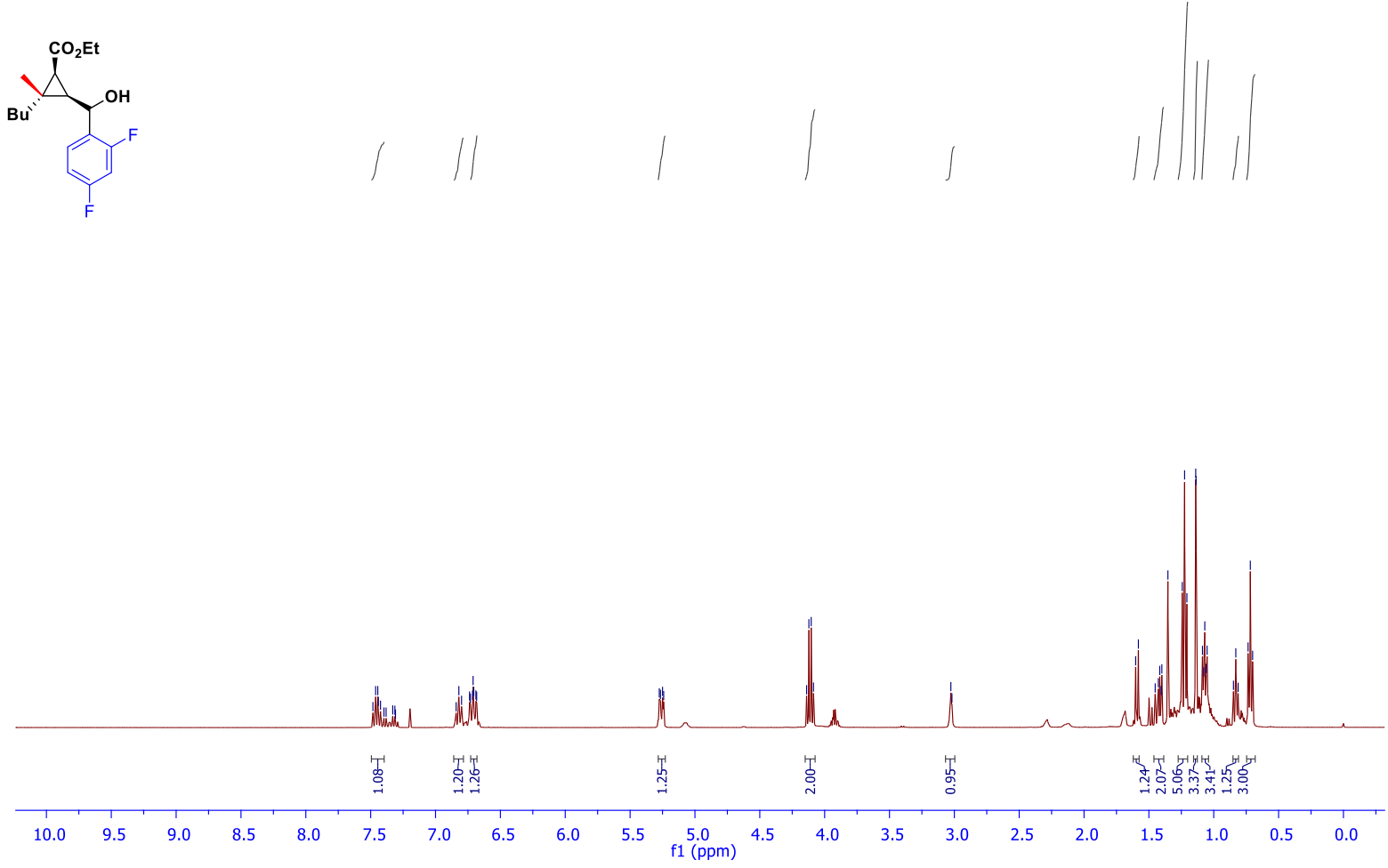

Veeru400

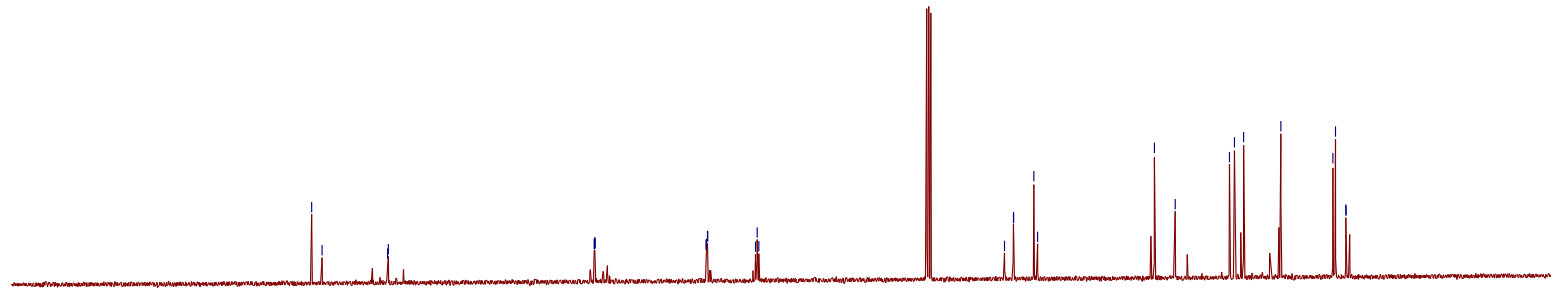

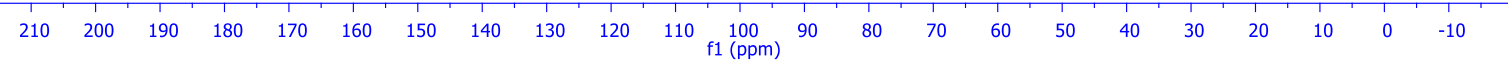


<smiles>CCC(=O)C1C(C(O)c2ccco2)C1(C)C</smiles>
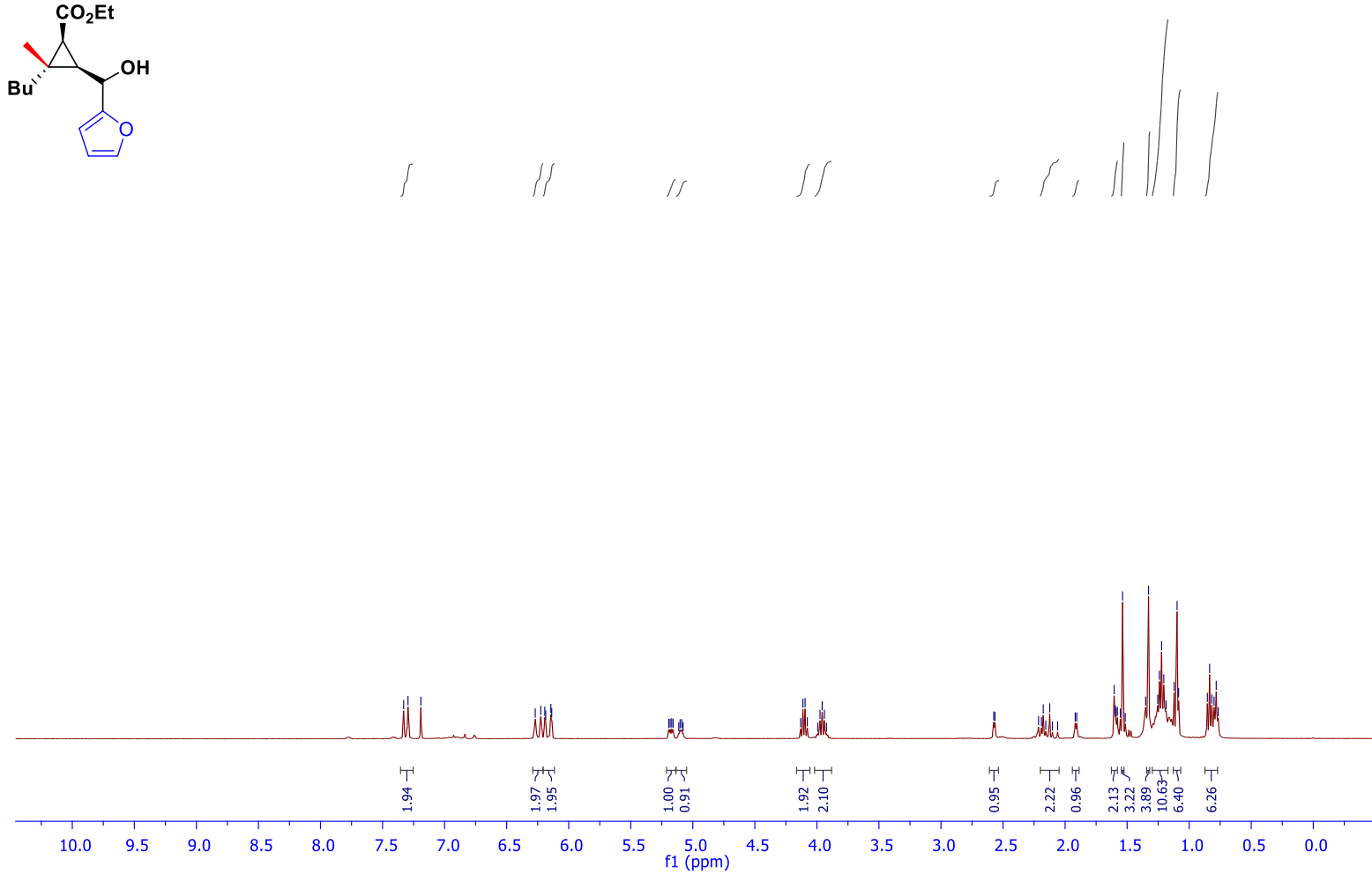

Veeru400

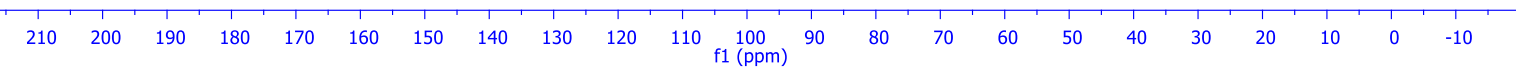


Veeru400

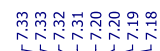

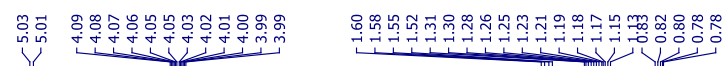

$\bigcup_{B u}^{\mathrm{CO}_{2} \mathrm{Et}}$
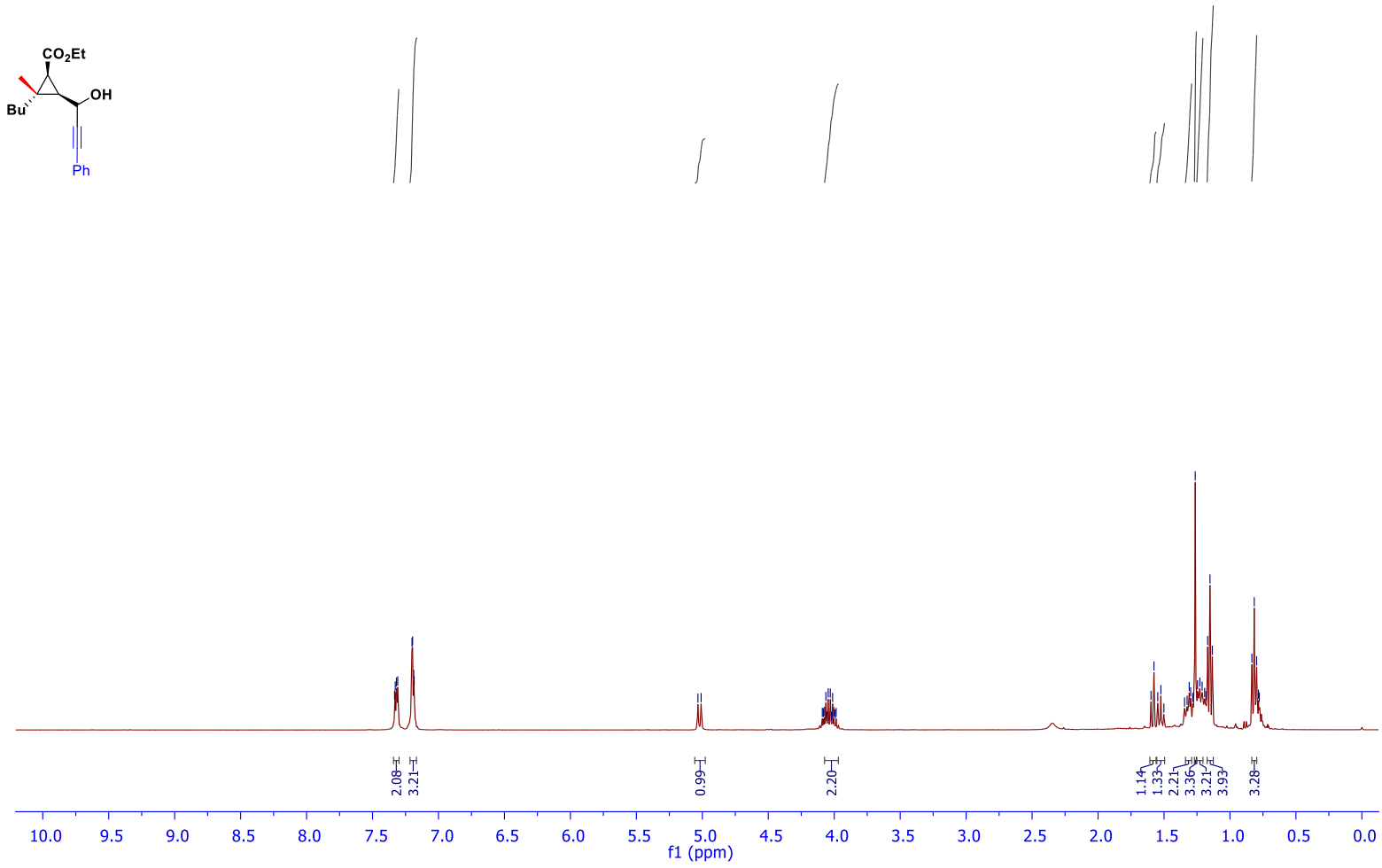

Veeru400

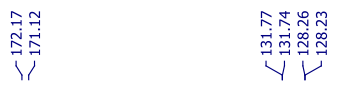

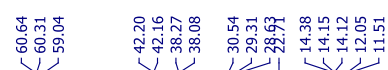

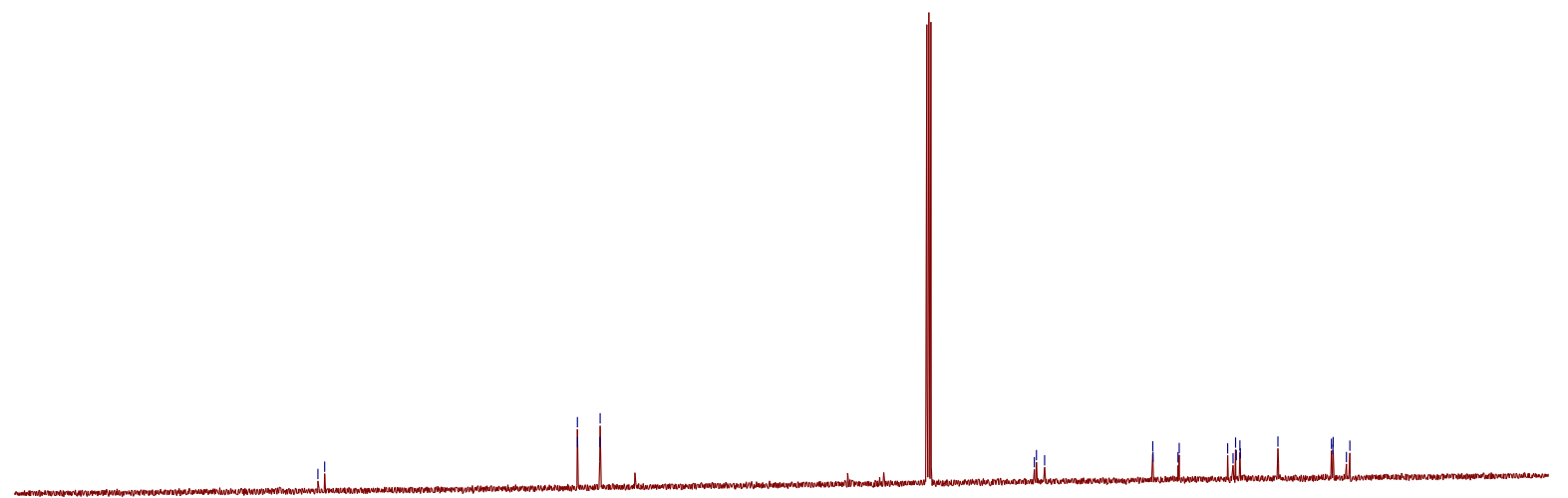

$\begin{array}{lllllllllllllllllllllllllllllll}210 & 200 & 190 & 180 & 170 & 160 & 150 & 140 & 130 & 120 & 110 & \begin{array}{c}100 \\ \mathrm{f} 1(\mathrm{ppm})\end{array} & 90 & 80 & 70 & 60 & 50 & 40 & 30 & 20 & 10 & 0 & -10\end{array}$ 


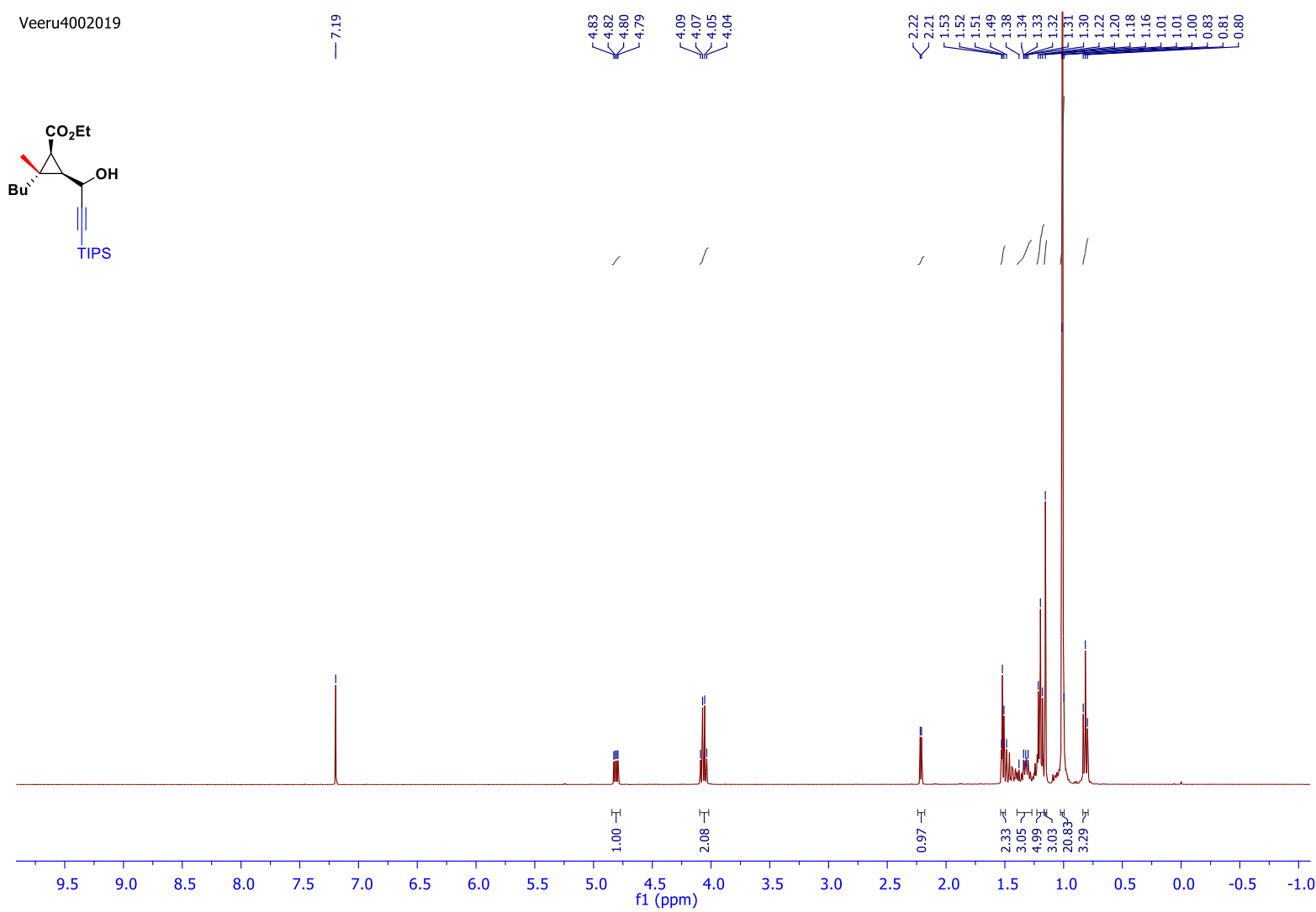

Veeru4002019

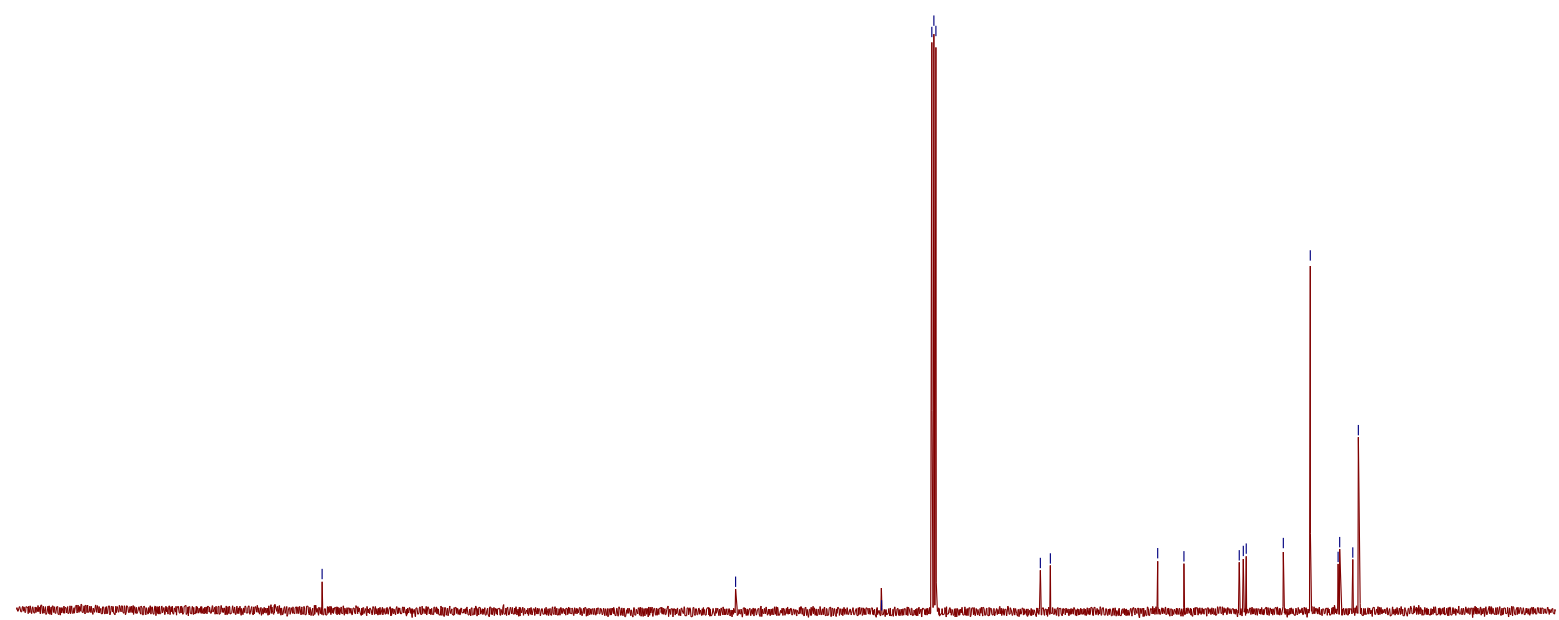

$\begin{array}{llllllllllllllllllllllllllll}210 & 200 & 190 & 180 & 170 & 160 & 150 & 140 & 130 & 120 & 110 & 100 & 100 & 80 & 70 & 60 & 50 & 40 & 30 & 20 & 10 & 0 & -10\end{array}$ 

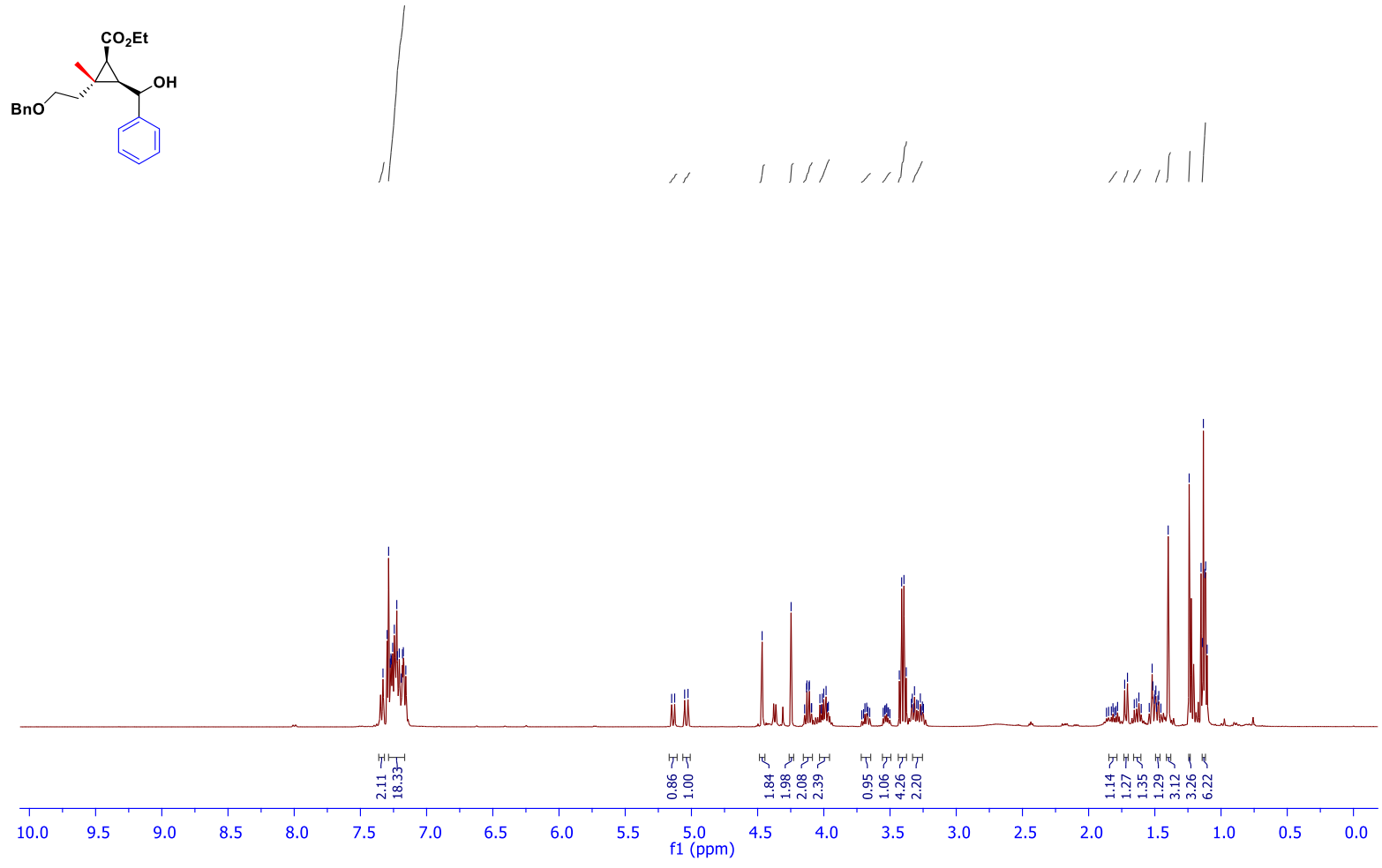

Veeru400

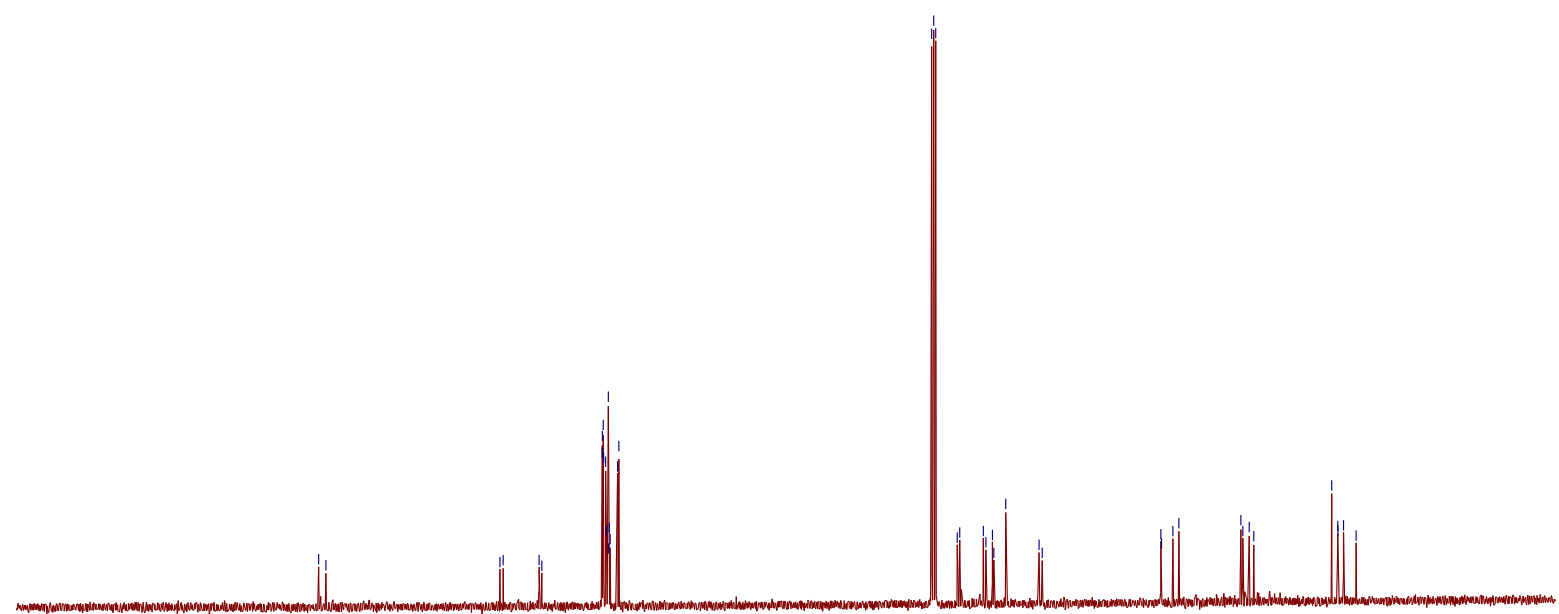

$\begin{array}{llllllllllllllllllllllllllll}210 & 200 & 190 & 180 & 170 & 160 & 150 & 140 & 130 & 120 & 110 & \underset{\mathrm{f} 1}{100}(\mathrm{ppm}) & 90 & 80 & 70 & 60 & 50 & 40 & 30 & 20 & 10 & 0 & -10\end{array}$ 
<smiles>C=C[C@H](O)C(C)(C)OCC</smiles>

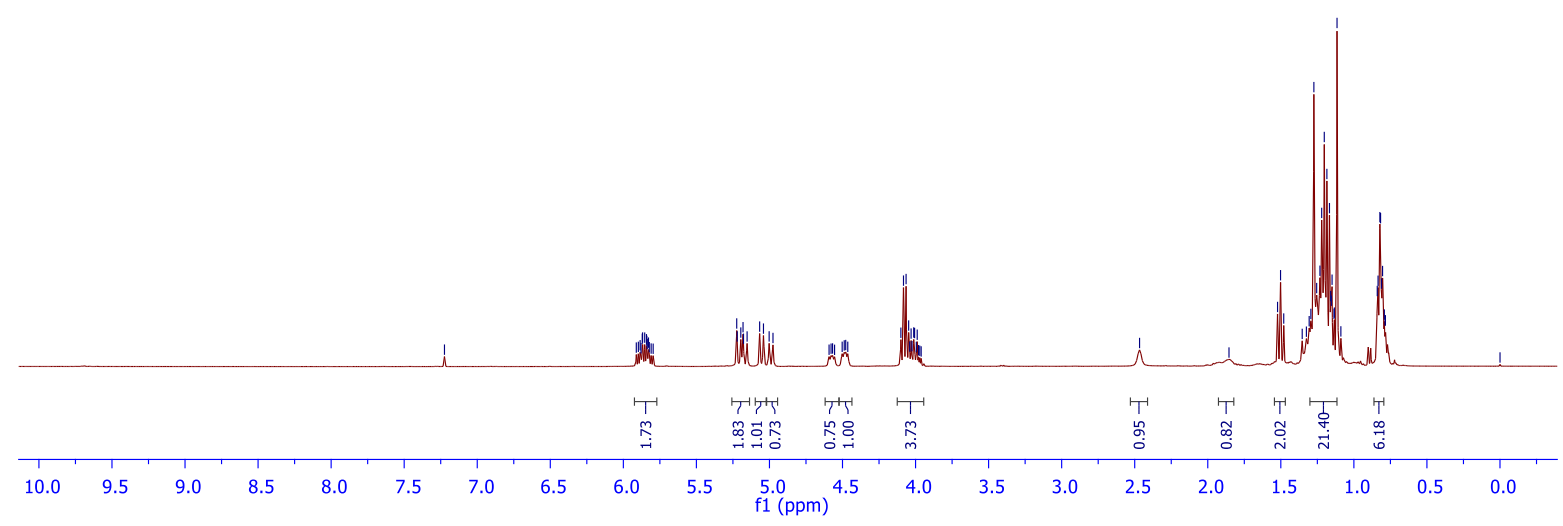

Veeru400

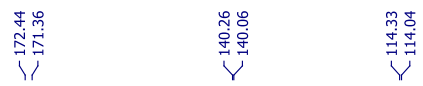

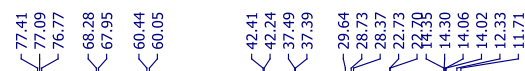

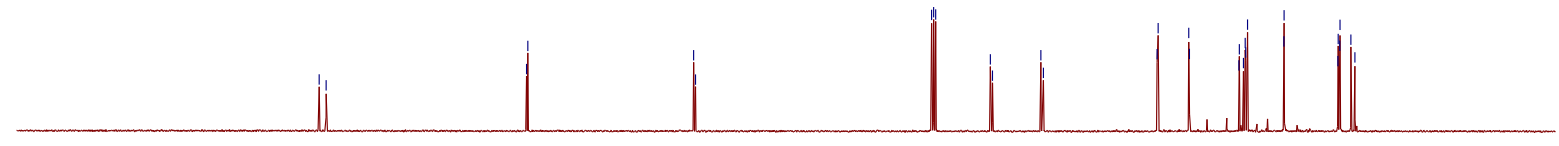

$\begin{array}{llllllllllllllllllllllllllllllll}210 & 200 & 190 & 180 & 170 & 160 & 150 & 140 & 130 & 120 & 110 & \begin{array}{c}100 \\ \mathrm{f} 1(\mathrm{ppm})\end{array} & 90 & 80 & 70 & 60 & 50 & 40 & 30 & 20 & 10 & 0 & -10\end{array}$ 
<smiles>CCOC(=O)C(C)[C@H](C)[C@H](O)c1ccc(C(O)C([13CH3])[14CH3])cc1</smiles>
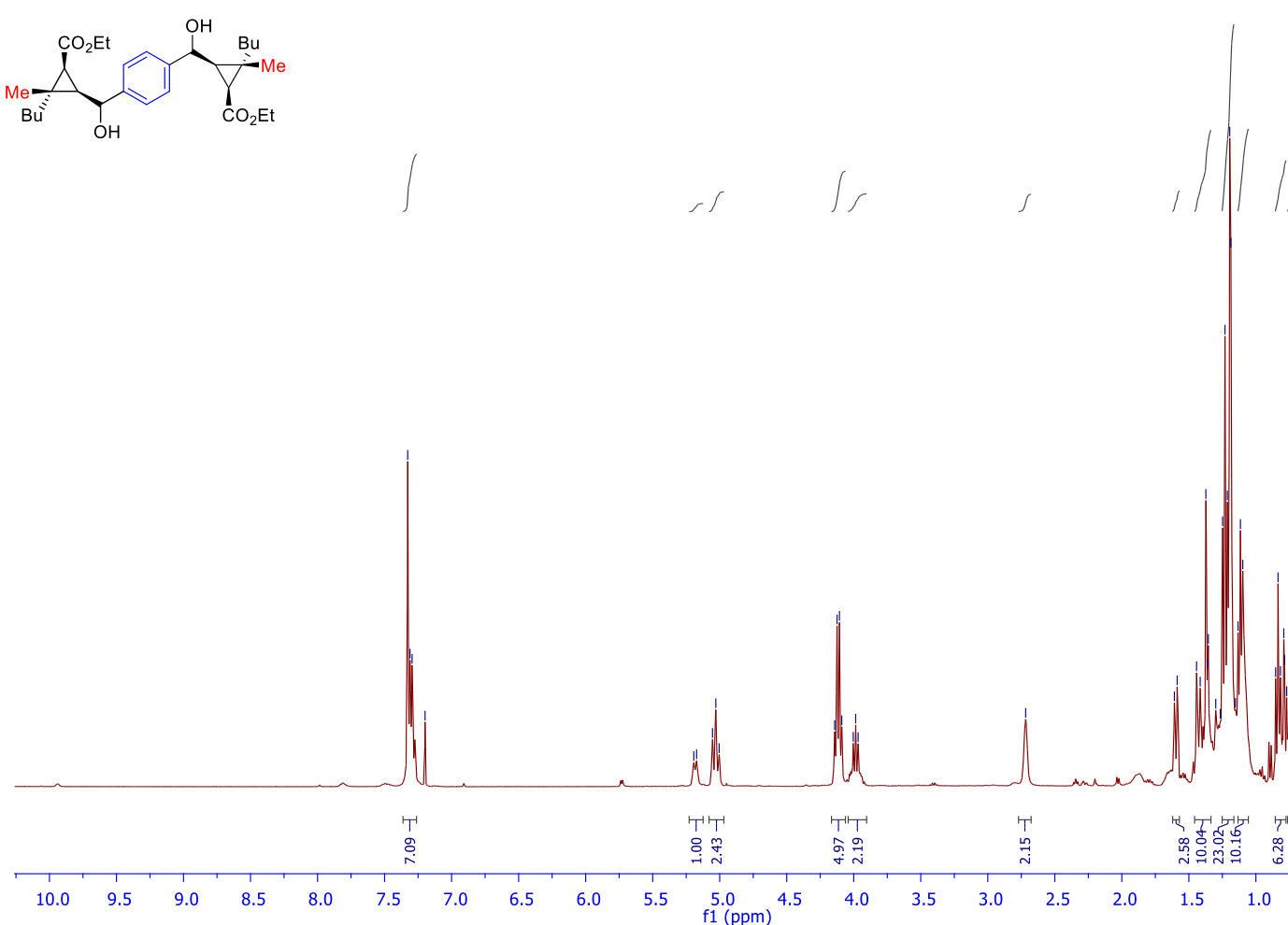

Veeru4002020

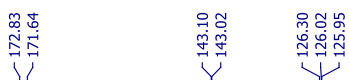

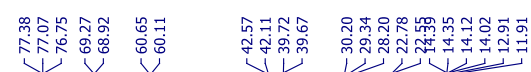

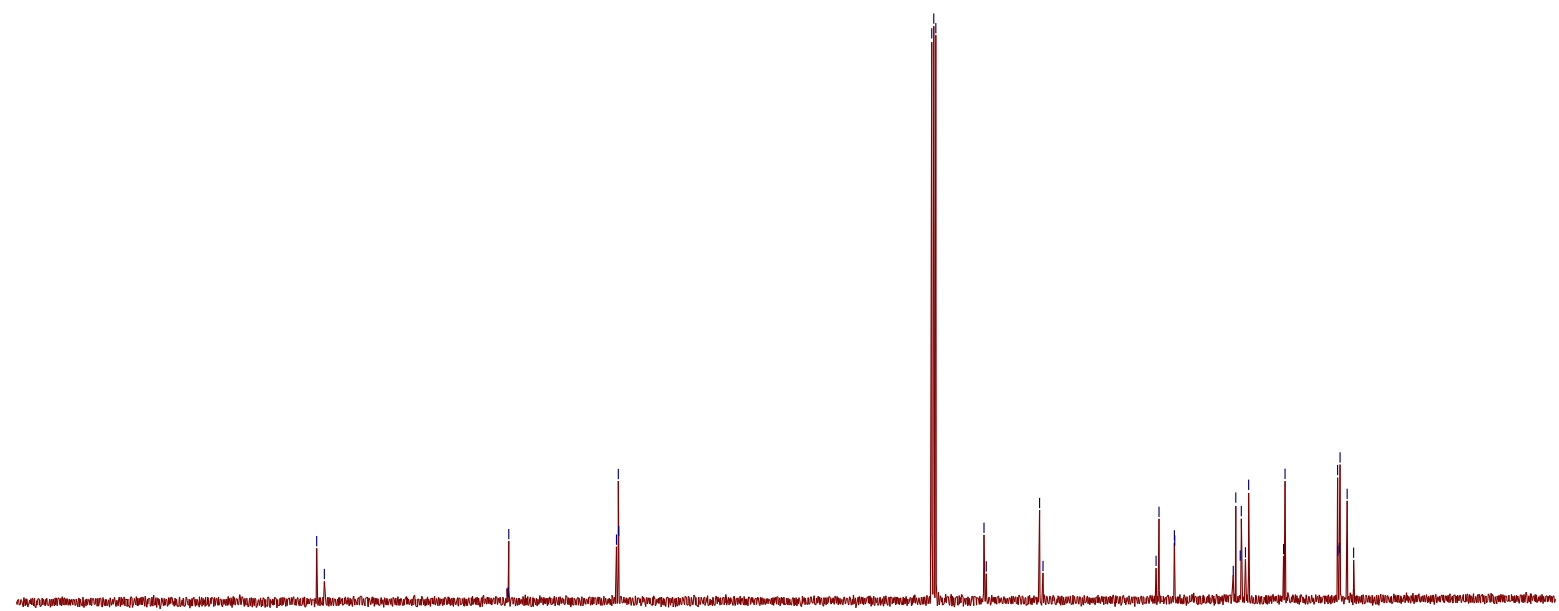

$\begin{array}{llllllllllllllllllllllllll}210 & 200 & 190 & 180 & 170 & 160 & 150 & 140 & 130 & 120 & 110 & \begin{array}{c}100 \\ 100\end{array} & 90 & 80 & 70 & 60 & 50 & 40 & 30 & 20 & 10 & 0 & -10\end{array}$ 


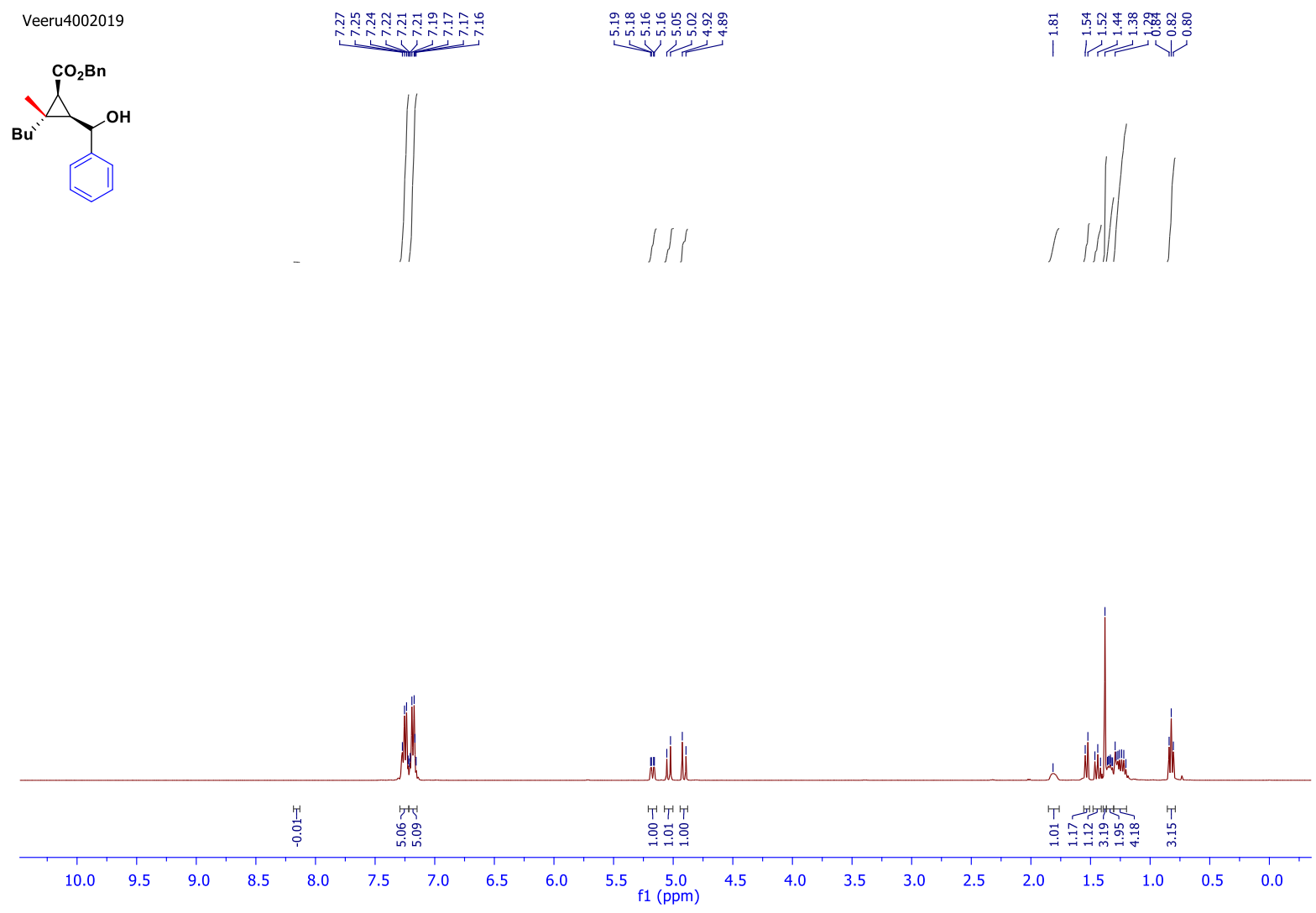

Veeru4002019

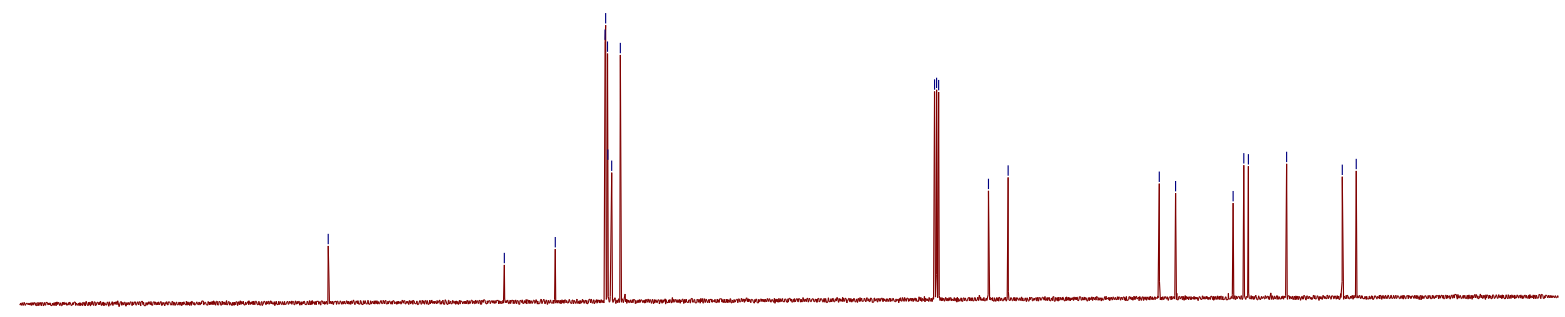


Veeru400

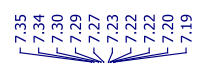

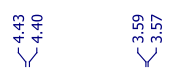

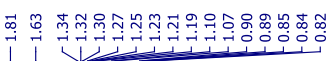

$\underbrace{\mathrm{OH}}$

$\|$

Is $\mid$

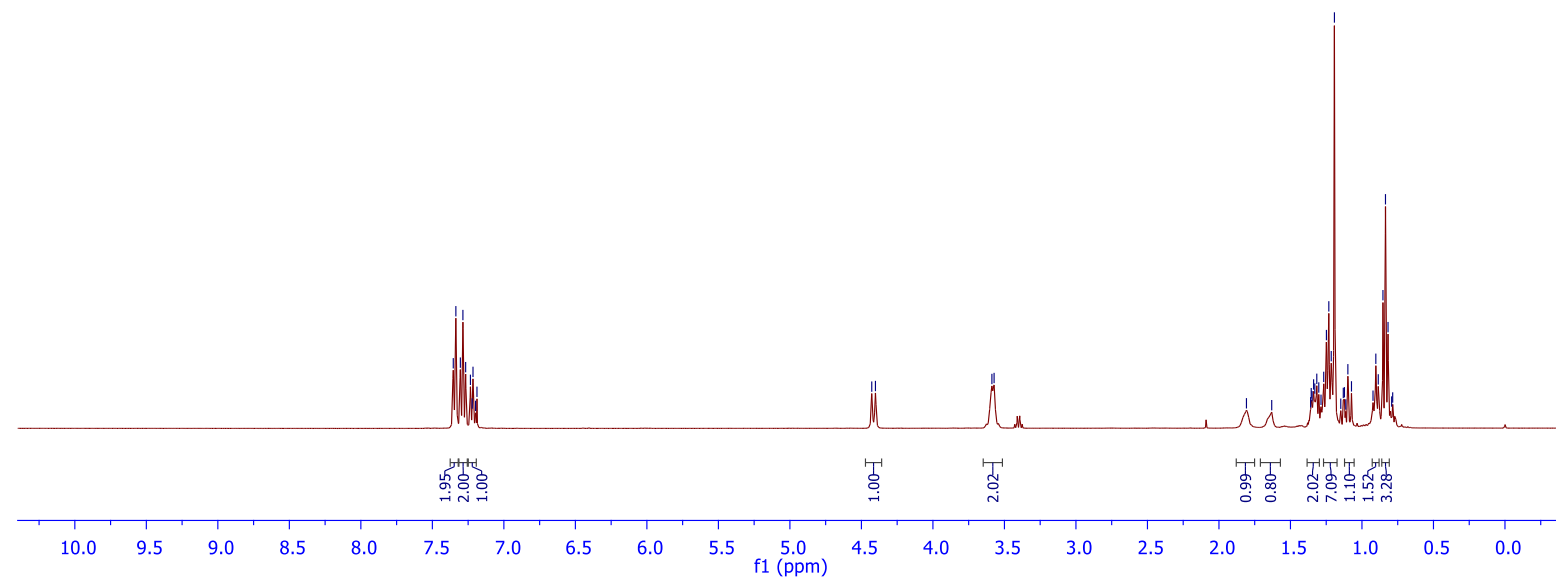

Veeru400

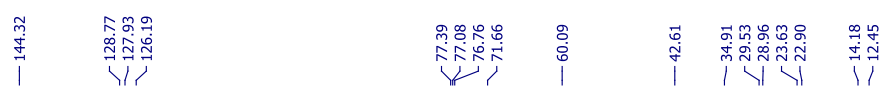

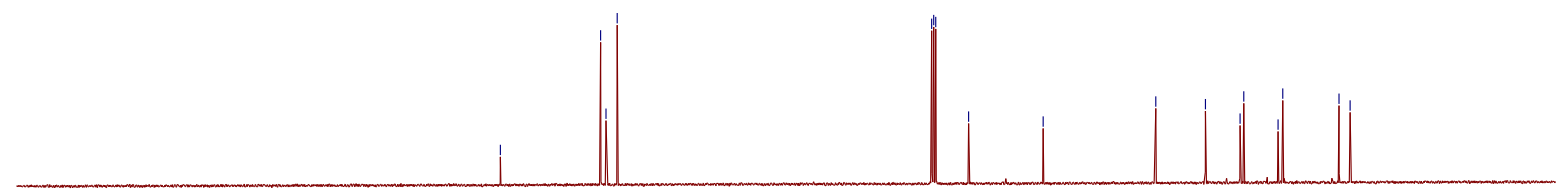

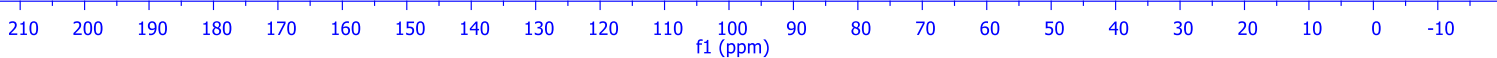



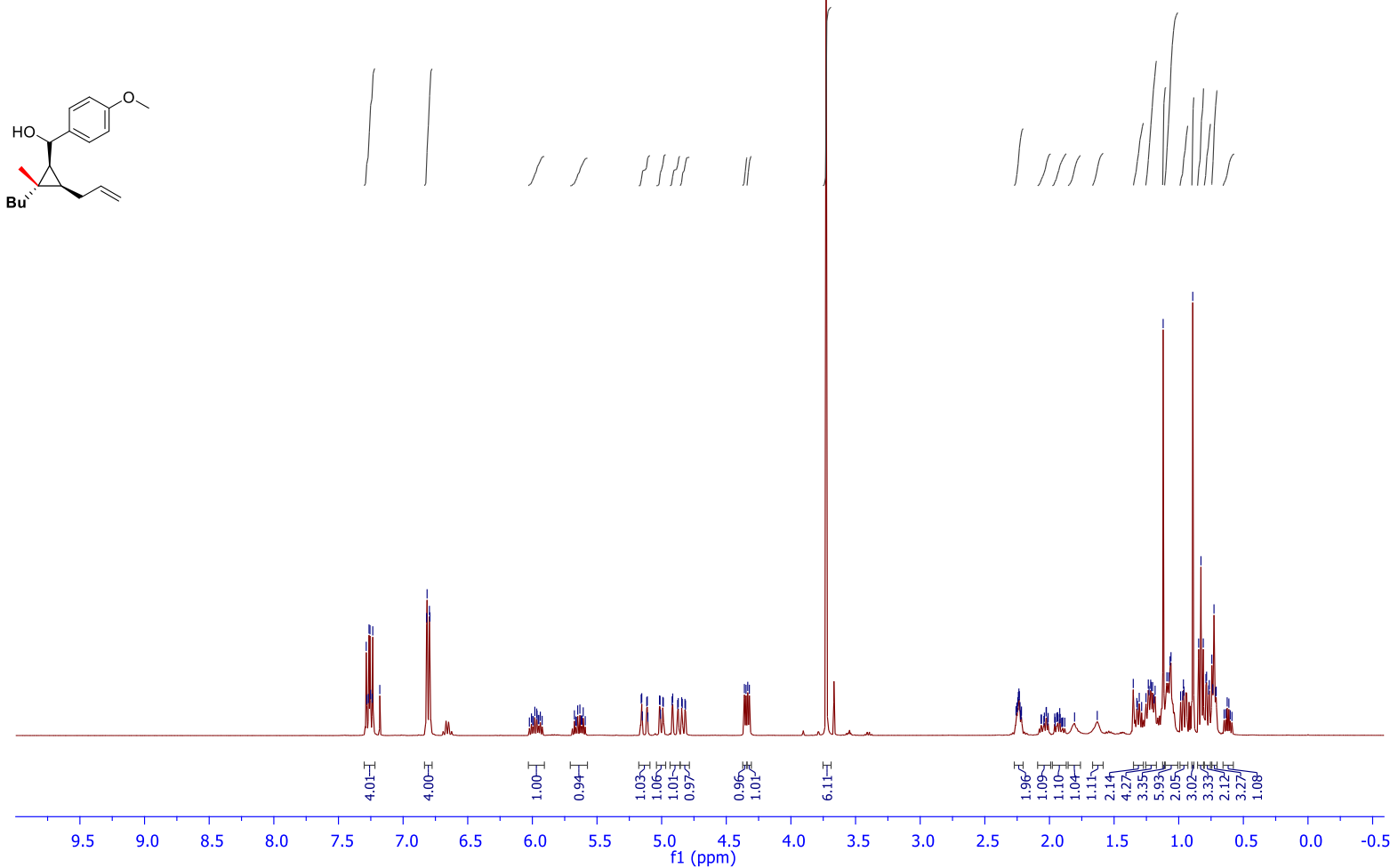

Veeru4002019

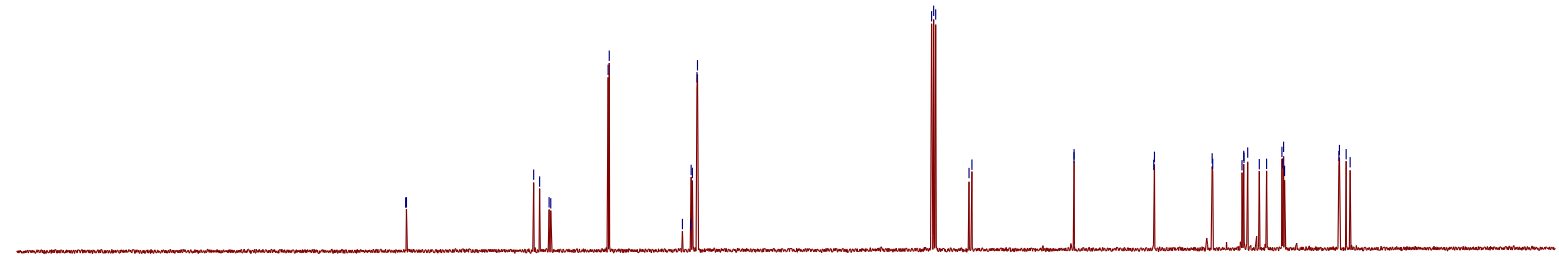

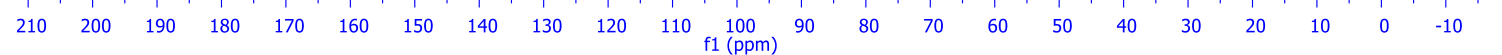


<smiles>CC(C)C(O)(O)c1ccc(O)cc1</smiles><smiles>C=CC1CCC1</smiles>

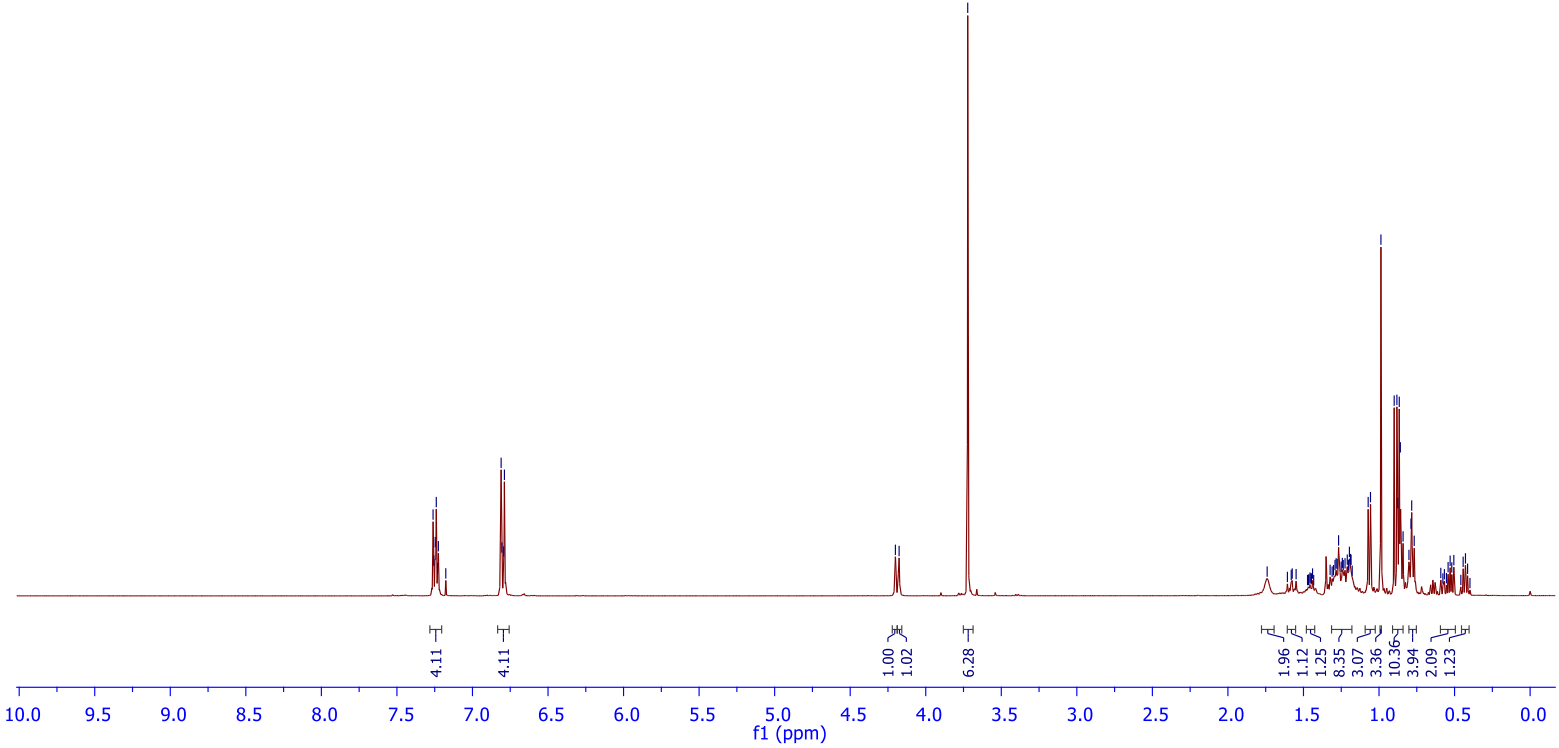

Veeru4002019

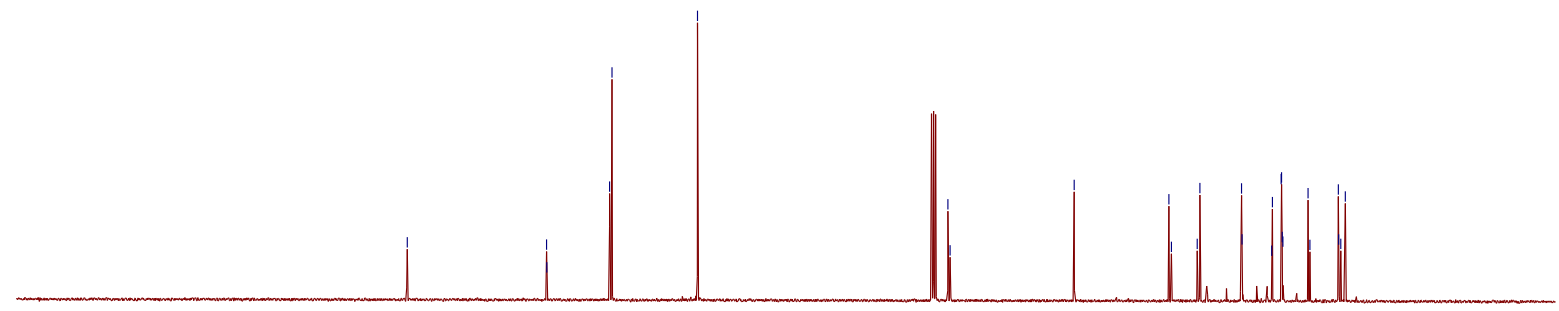

$\begin{array}{llllllllllllllllllllllllll}210 & 200 & 190 & 180 & 170 & 160 & 150 & 140 & 130 & 120 & 110 & \begin{array}{c}100 \\ 100\end{array} & 90 & 80 & 70 & 60 & 50 & 40 & 30 & 20 & 10 & 0 & -10\end{array}$ 


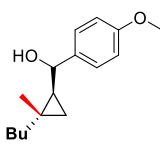
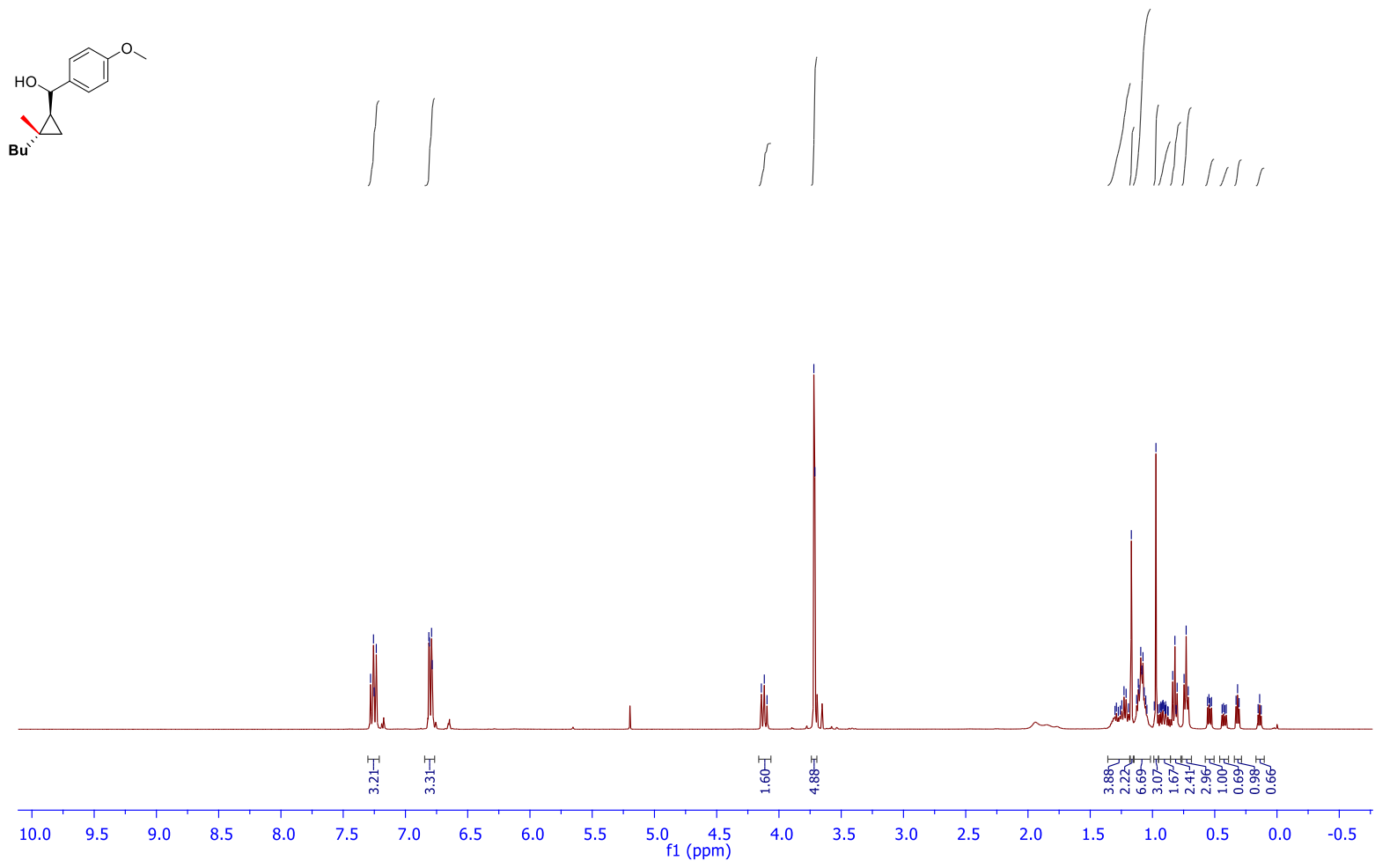

Veeru4002019

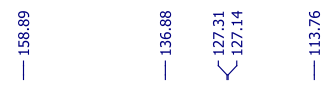

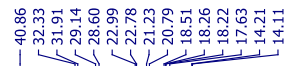

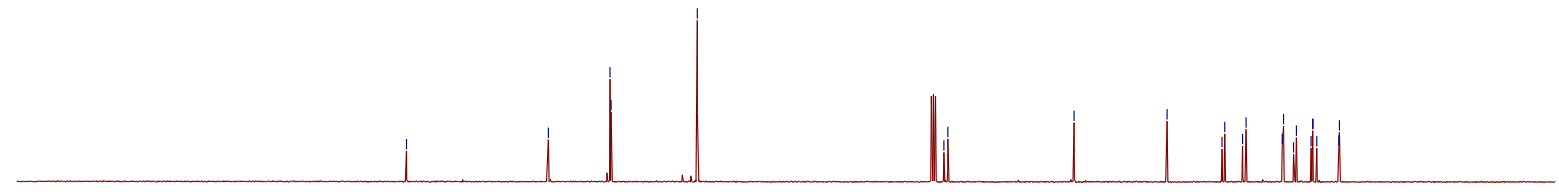

$\begin{array}{llllllllllllllllllllllllll}210 & 200 & 190 & 180 & 170 & 160 & 150 & 140 & 130 & 120 & 110 & \begin{array}{c}100 \\ \mathrm{f} 1(\mathrm{ppm})\end{array} & 90 & 80 & 70 & 60 & 50 & 40 & 30 & 20 & 10 & 0 & -10\end{array}$ 
Veeru4002019

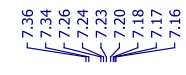

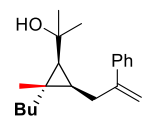

品触

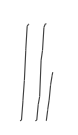

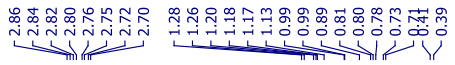

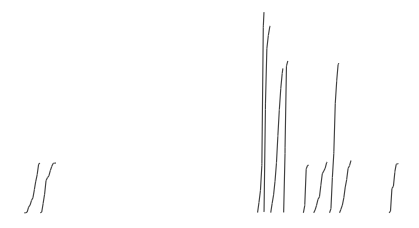

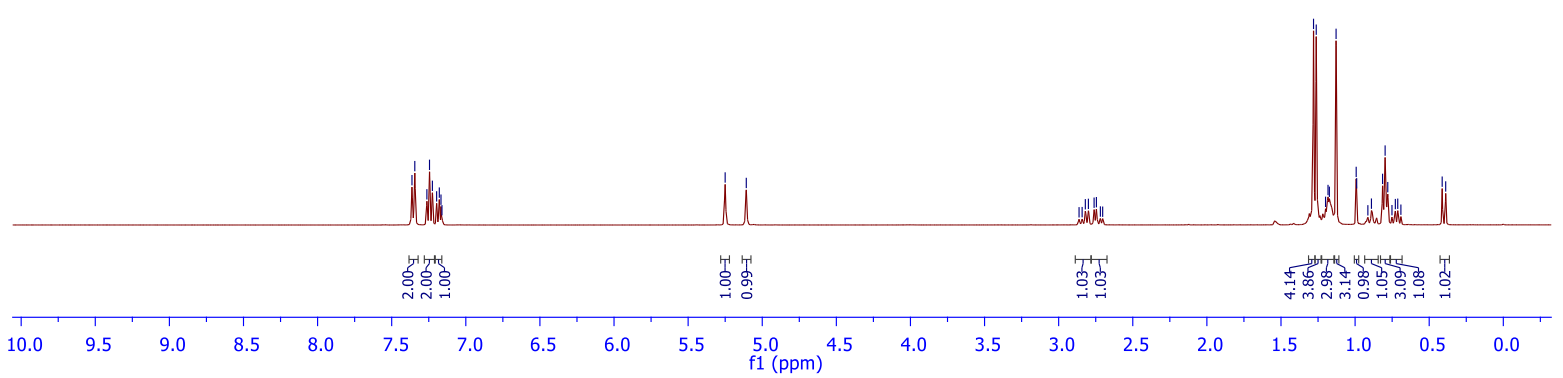

Veeru4002019
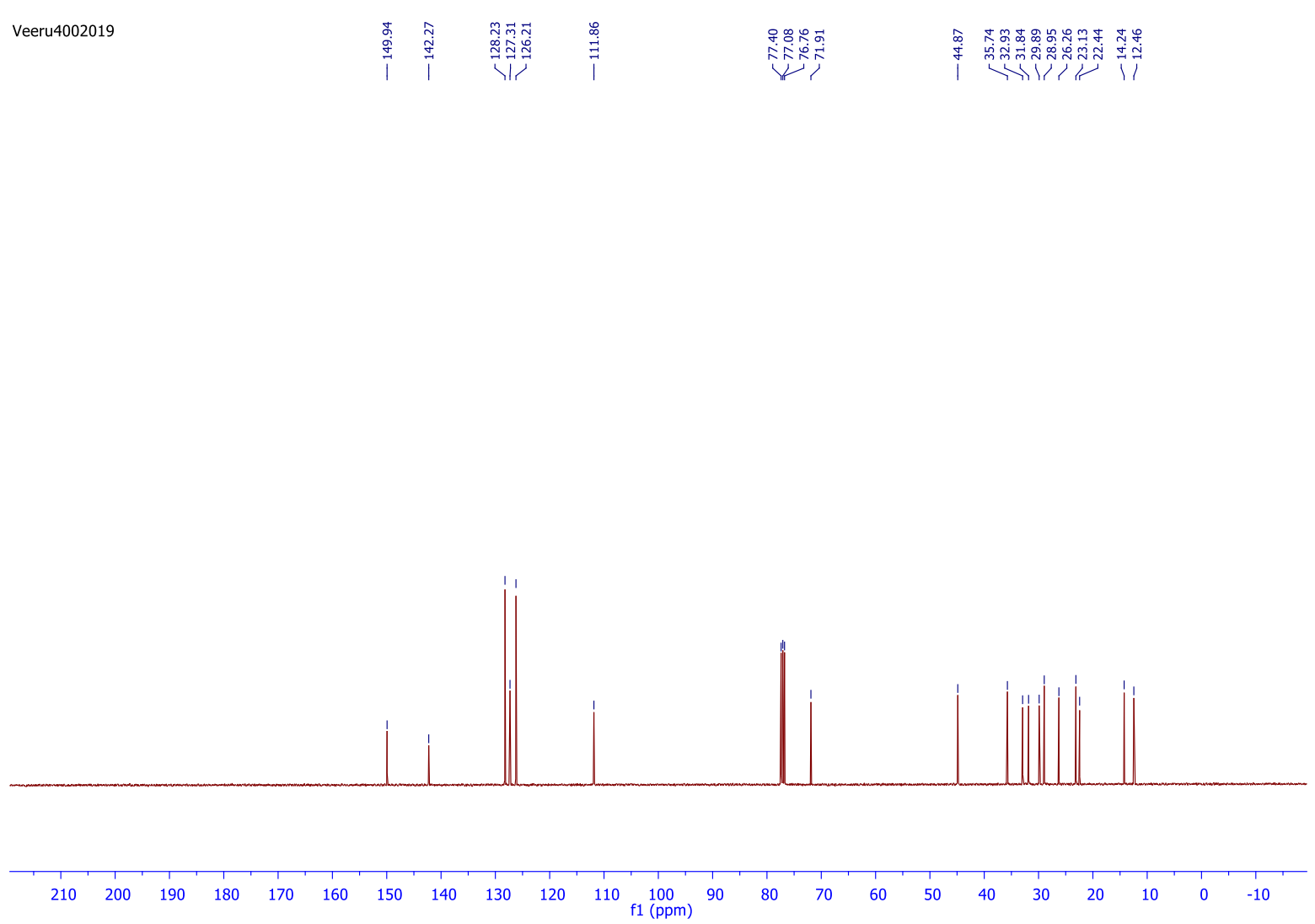

SI-39 
Veeru4002019

ำ

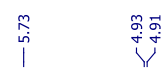

ํํำ

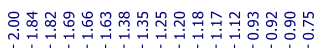

$\underbrace{\mathrm{Ph}}_{\mathrm{OH}}$

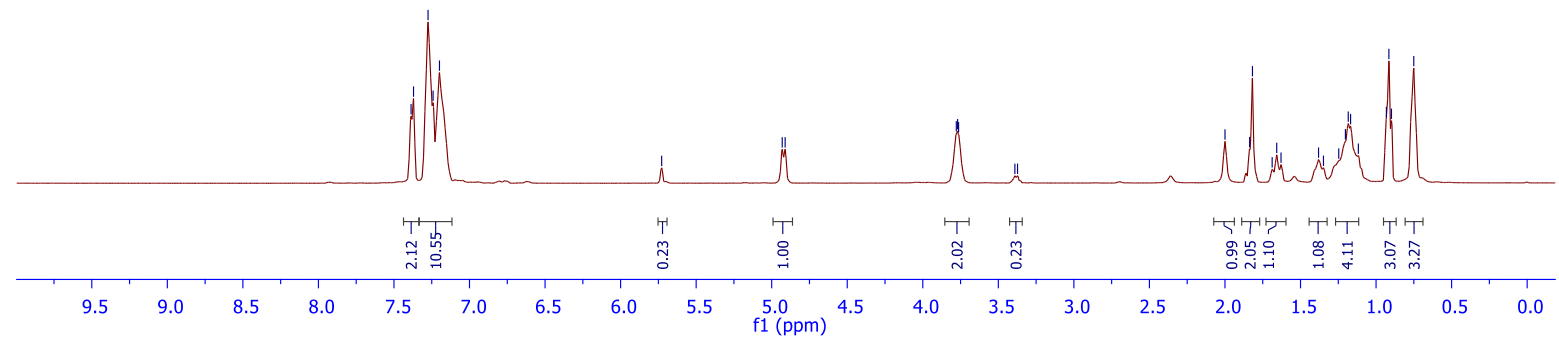

Veeru4002019

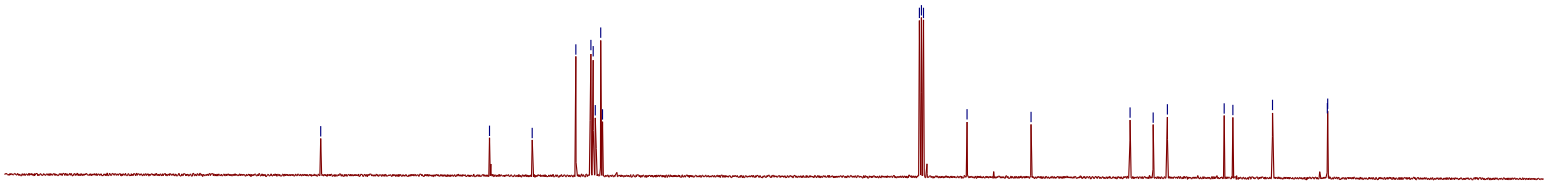

$\begin{array}{lllllllllllllllllllllllllll}210 & 200 & 190 & 180 & 170 & 160 & 150 & 140 & 130 & 120 & 110 & 100 & 90 & 80 & 70 & 60 & 50 & 40 & 30 & 20 & 10 & 0 & -10\end{array}$ 
Veeru400

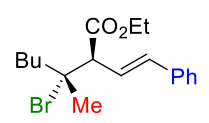

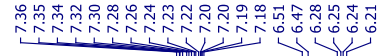

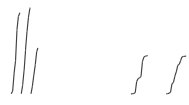

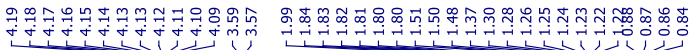

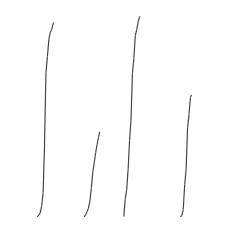

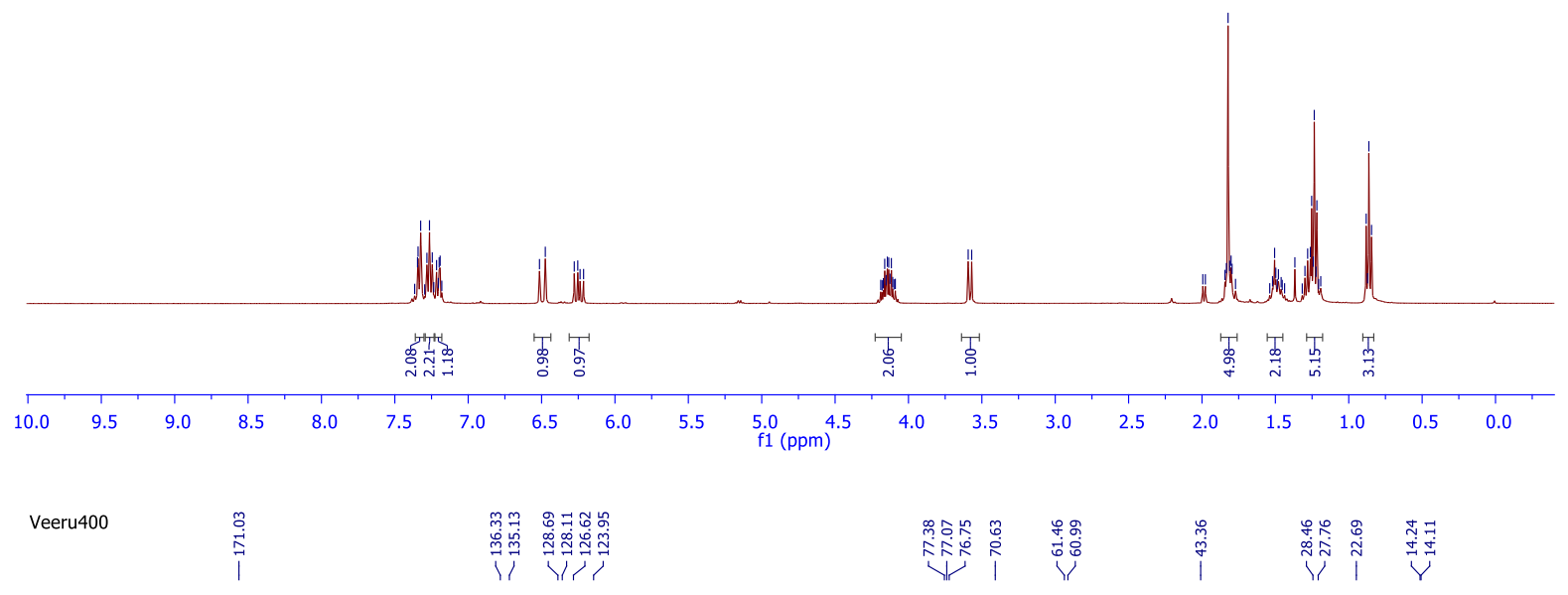
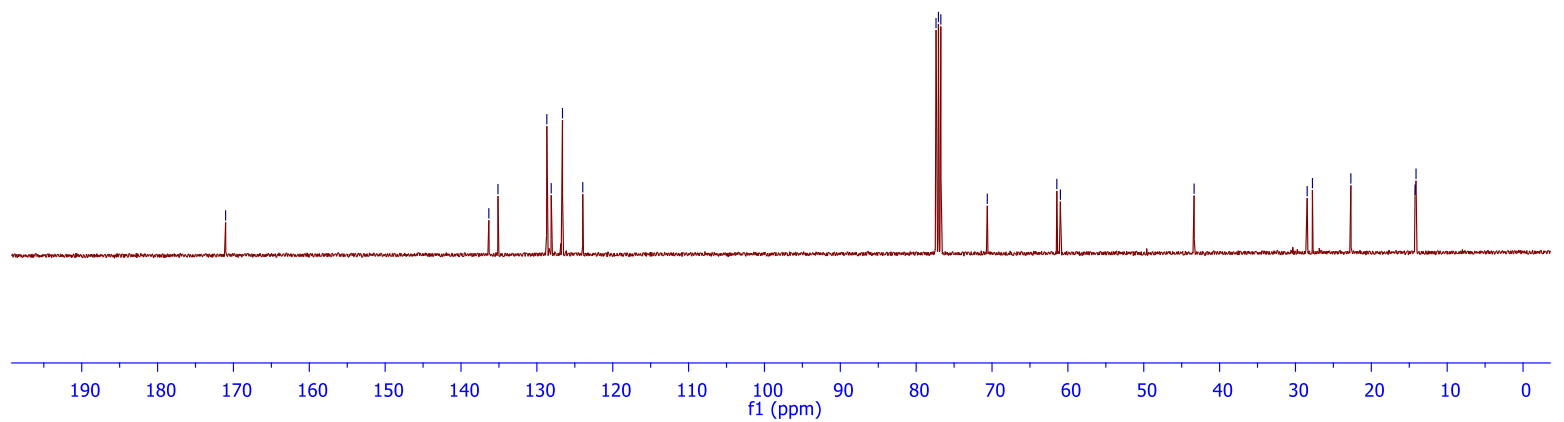

SI-41 
Veeru400
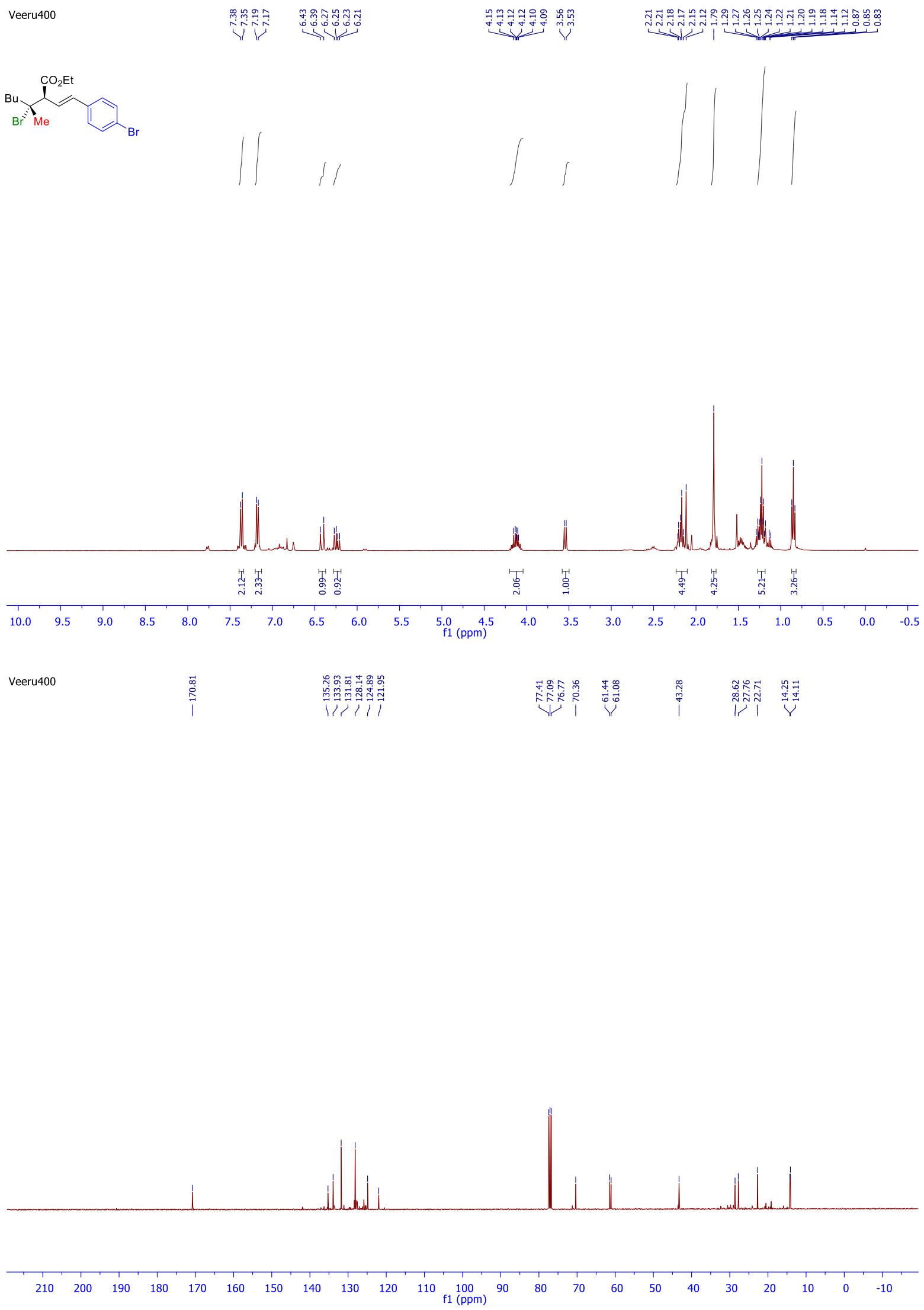

SI-42 
Veeru400<smiles>CCOC(=CC=Cc1ccc2c(c1)OCO2)C([NH3+])(Br)C(Br)(Br)Br</smiles>

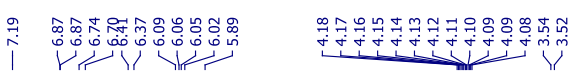

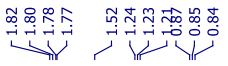

III / /
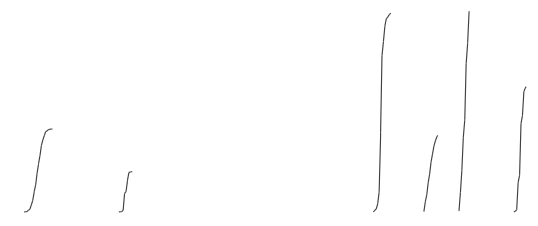

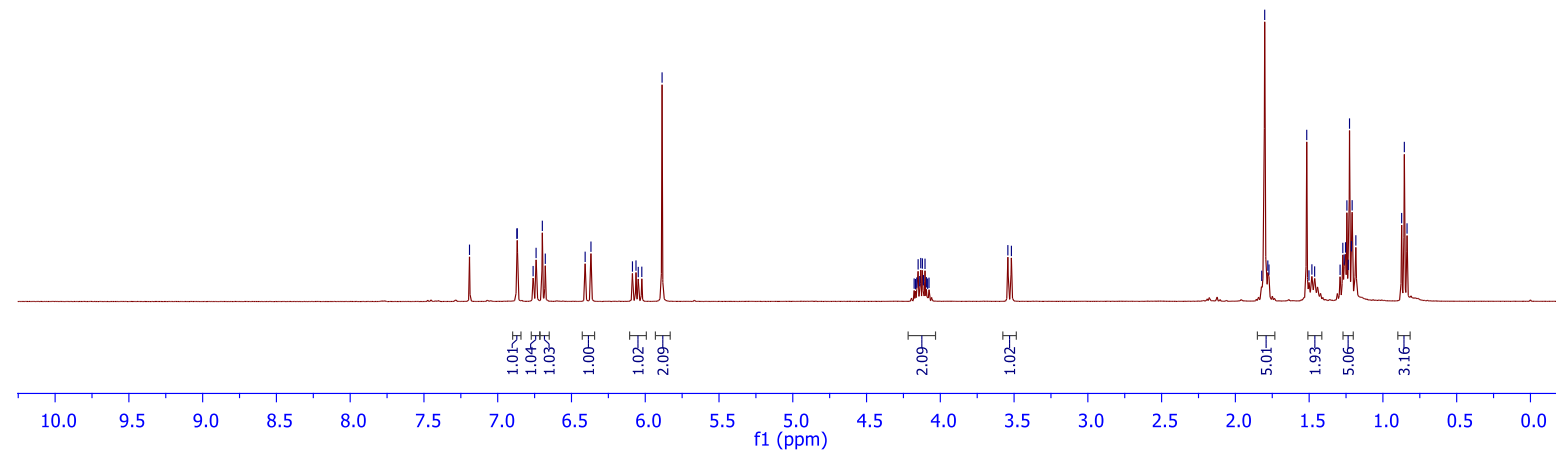

Veeru400

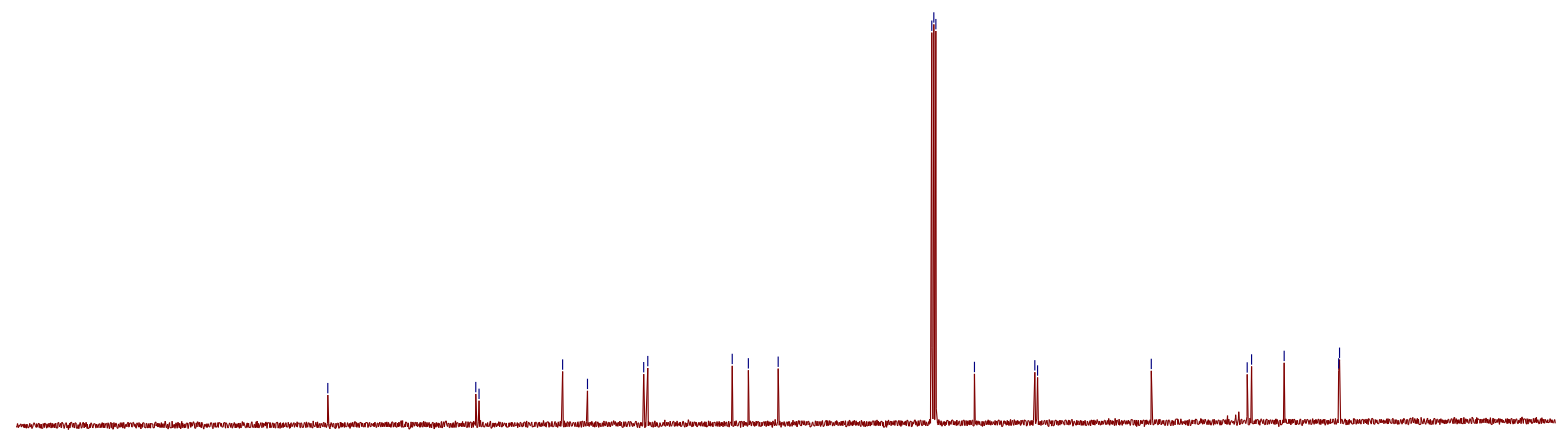

$\begin{array}{llllllllllllllllllllllll}210 & 200 & 190 & 180 & 170 & 160 & 150 & 140 & 130 & 120 & 110 & 100 & 100 & 80 & 70 & 60 & 50 & 40 & 30 & 20 & 10 & 0 & -10\end{array}$ 
<smiles>[Y4]C(Br)(Br)C(/C=C/c1ccccc1)C(=O)OCC</smiles>

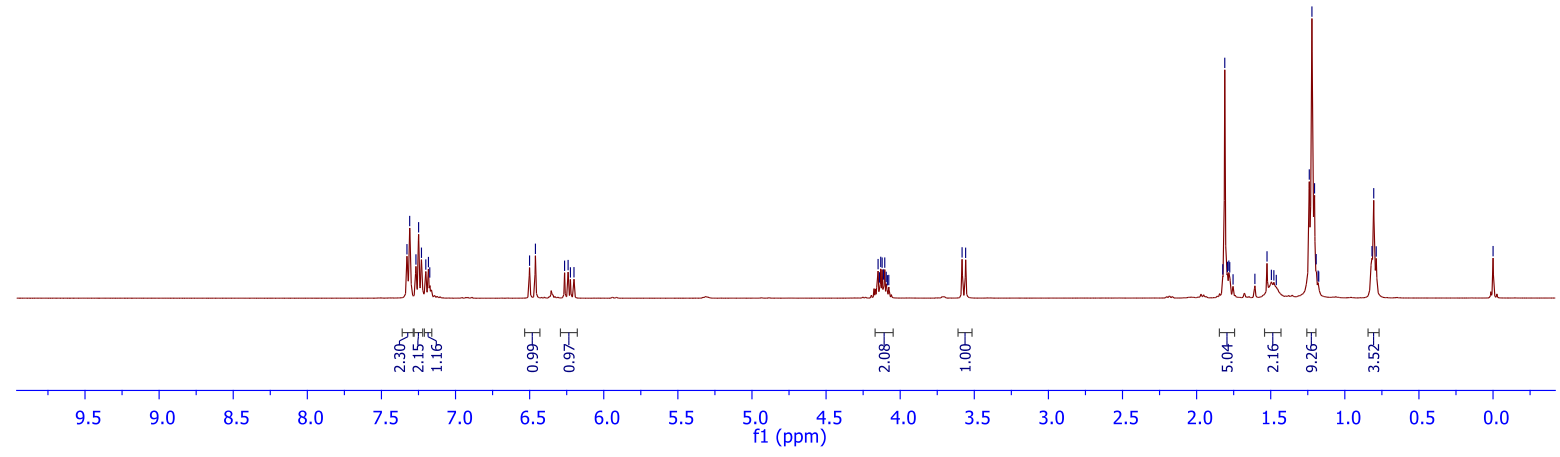

Veeru400

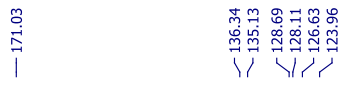

倸।

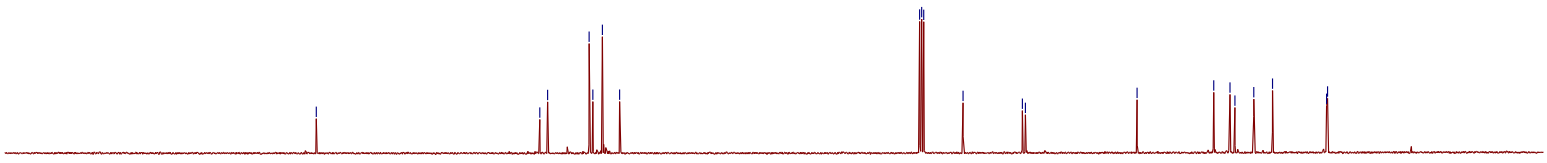

$\begin{array}{lllllllllllllllllllllllllllll}210 & 200 & 190 & 180 & 170 & 160 & 150 & 140 & 130 & 120 & 110 & 100 & 100 & 80 & 70 & 60 & 50 & 40 & 30 & 20 & 10 & 0 & -10\end{array}$ 


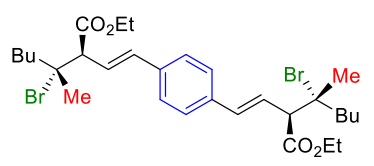
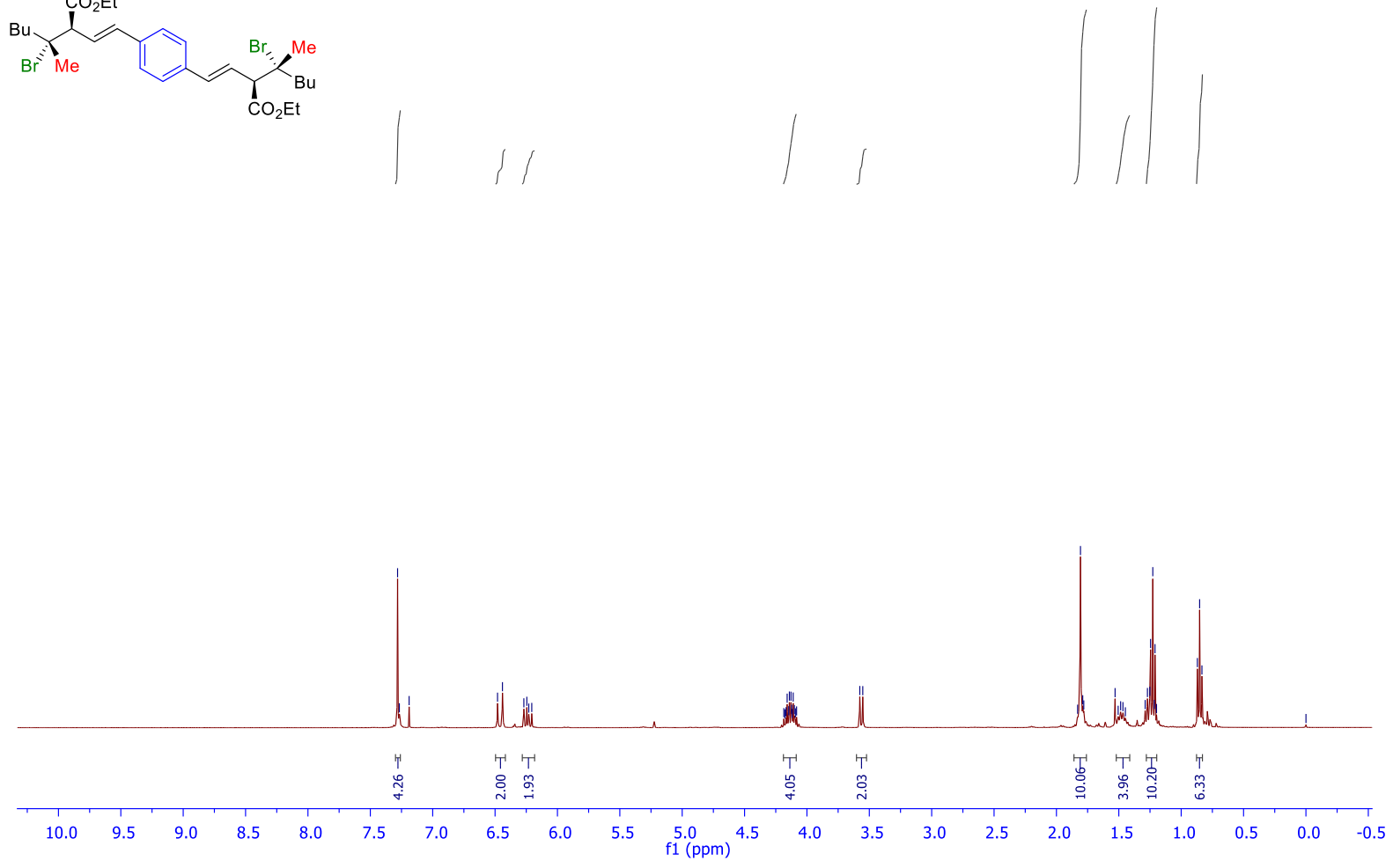

Veeru4002019

|

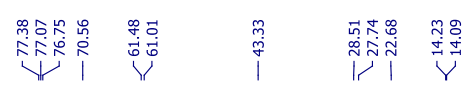

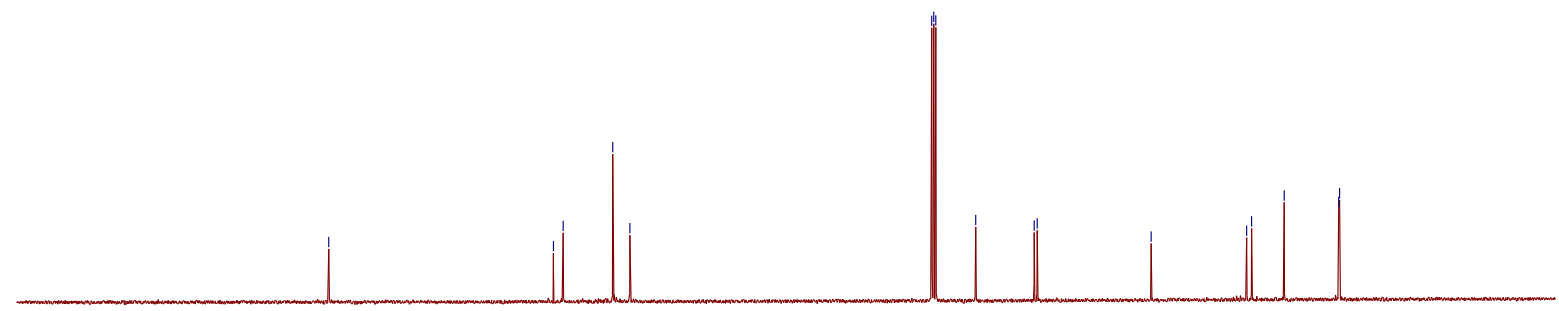

$\begin{array}{lllllllllllllllllllllllllll}210 & 200 & 190 & 180 & 170 & 160 & 150 & 140 & 130 & 120 & 110 & 100 & 90 & 80 & 70 & 60 & 50 & 40 & 30 & 20 & 10 & 0 & -10\end{array}$ 


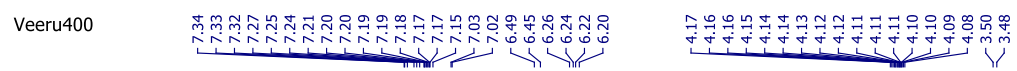

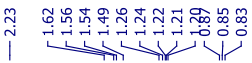

(il

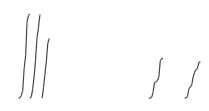

$\int 1$
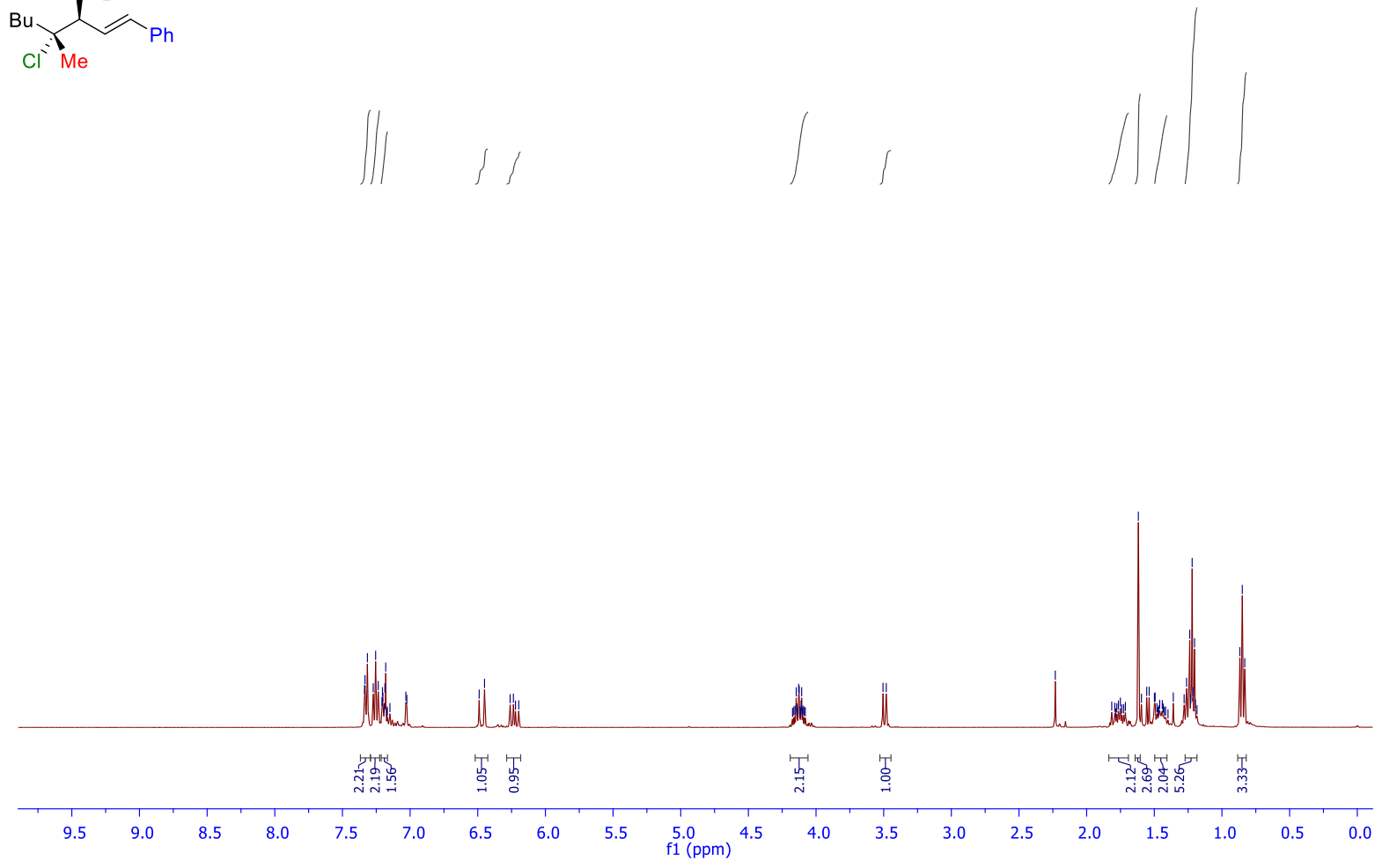

Veeru 400

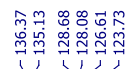

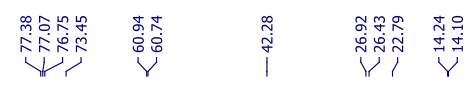

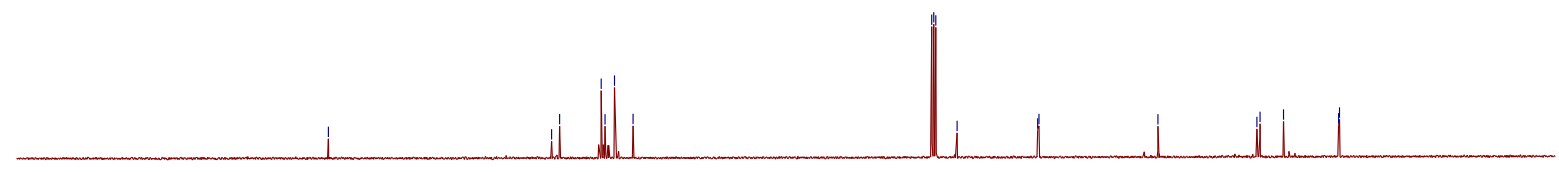

$\begin{array}{lllllllllllllllllllllllll}210 & 200 & 190 & 180 & 170 & 160 & 150 & 140 & 130 & 120 & 110 & 100 & 100 & 80 & 70 & 60 & 50 & 40 & 30 & 20 & 10 & 0 & -10\end{array}$ 

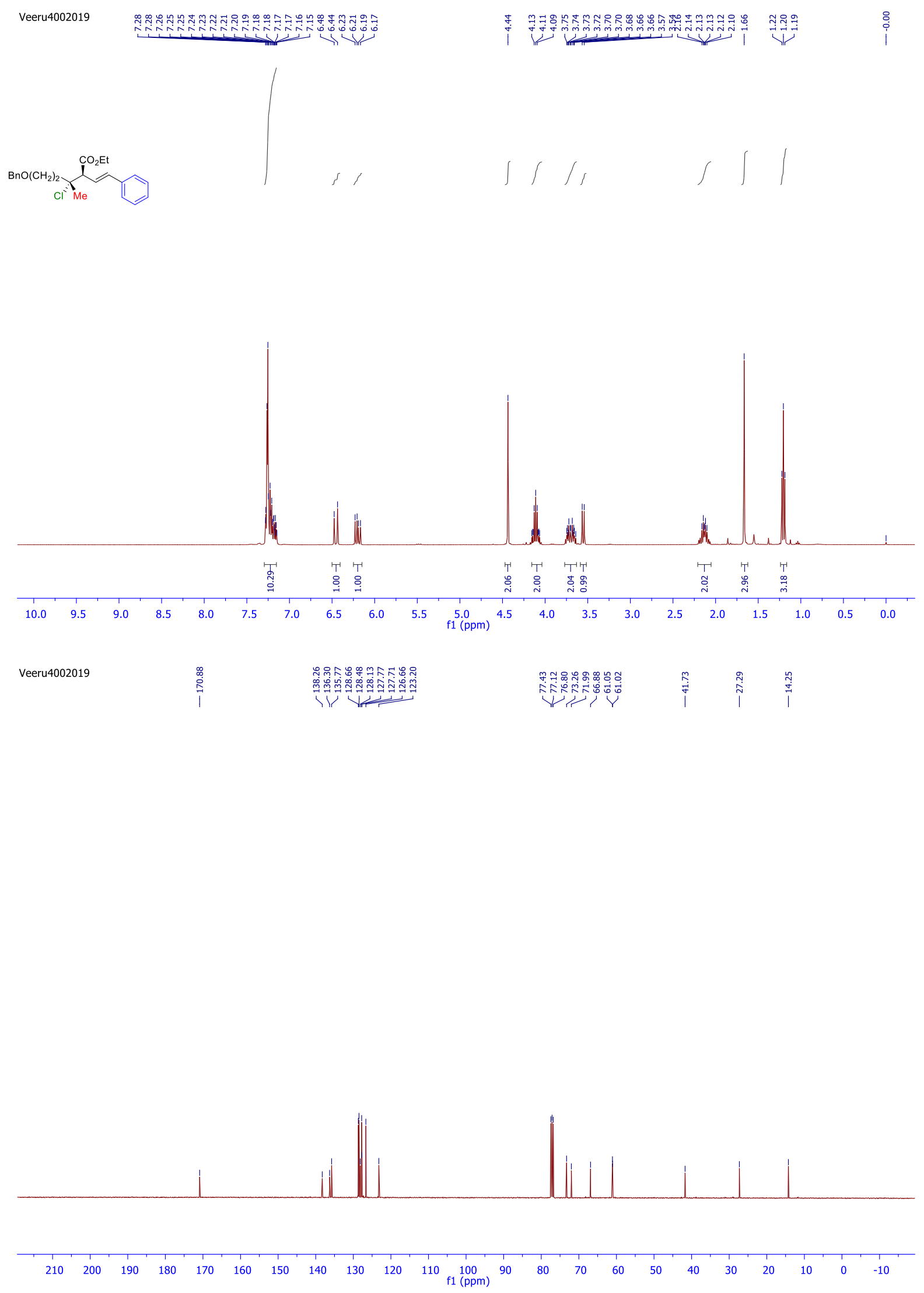

SI-48 
Veeru400

(li

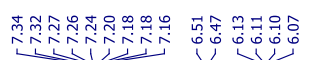

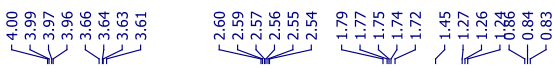

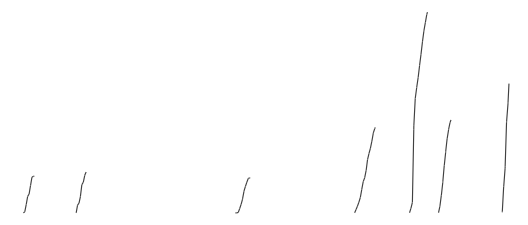

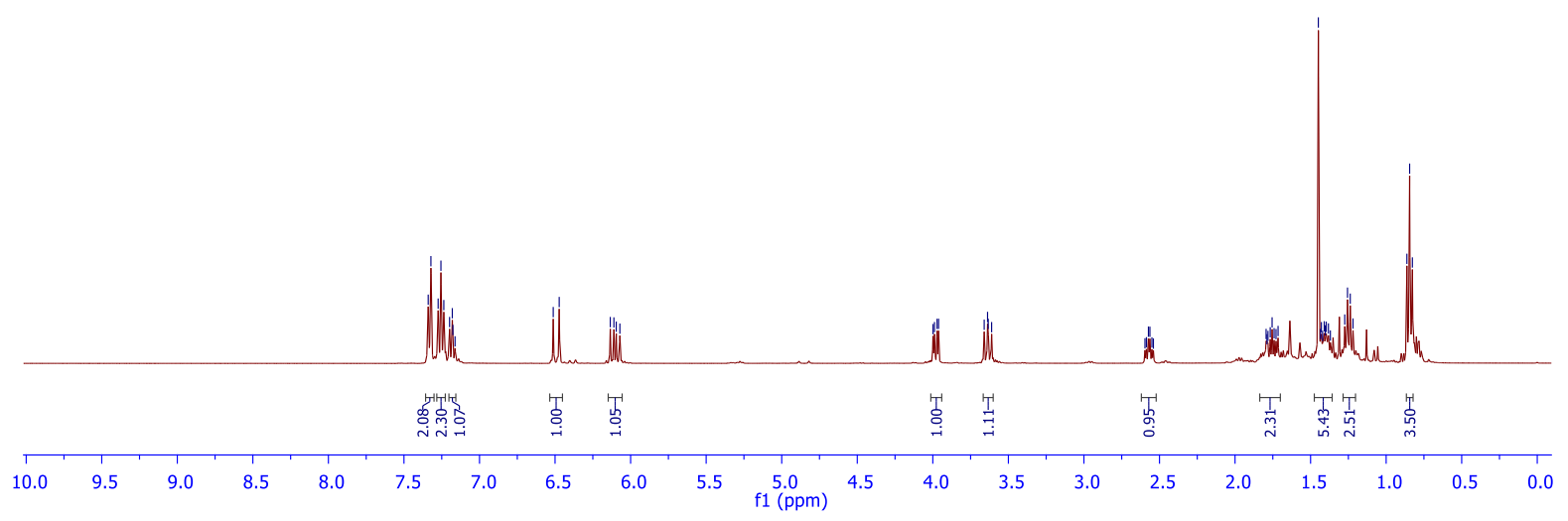

Veeru400

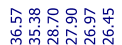

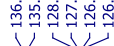

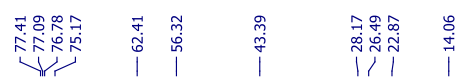

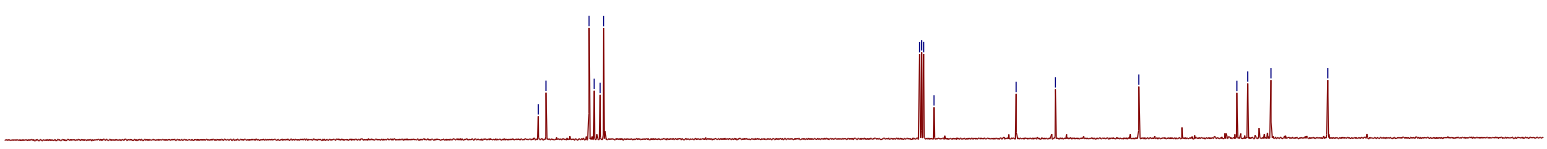

$\begin{array}{llllllllllllllllllllllllllll}210 & 200 & 190 & 180 & 170 & 160 & 150 & 140 & 130 & 120 & 110 & 100 & 100 & 80 & 70 & 60 & 50 & 40 & 30 & 20 & 10 & 0 & -10\end{array}$ 


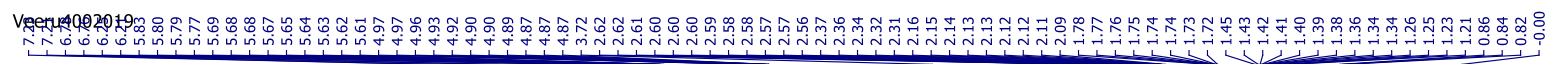
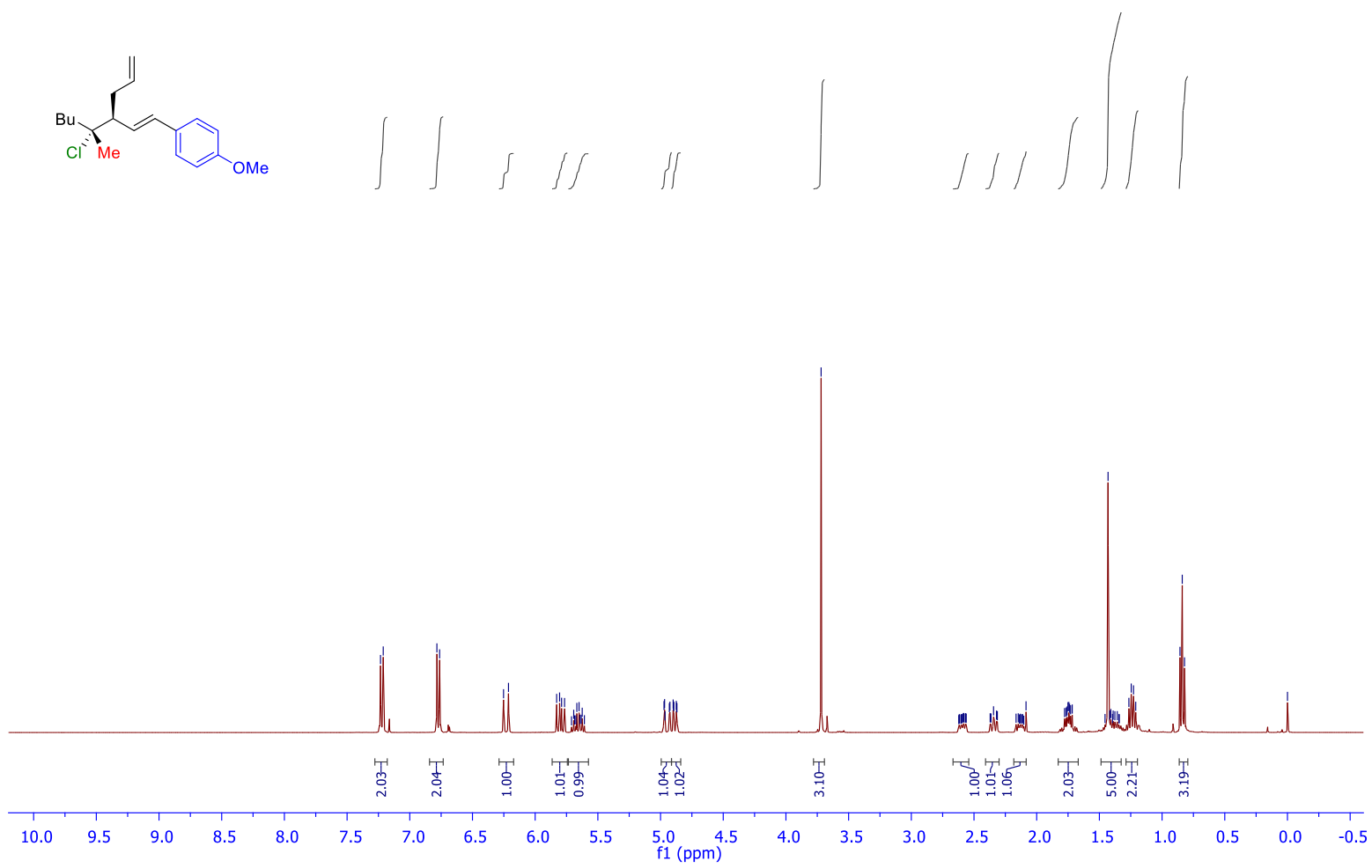

Veeru4002019

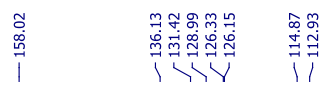

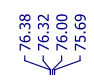

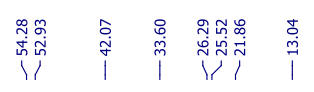
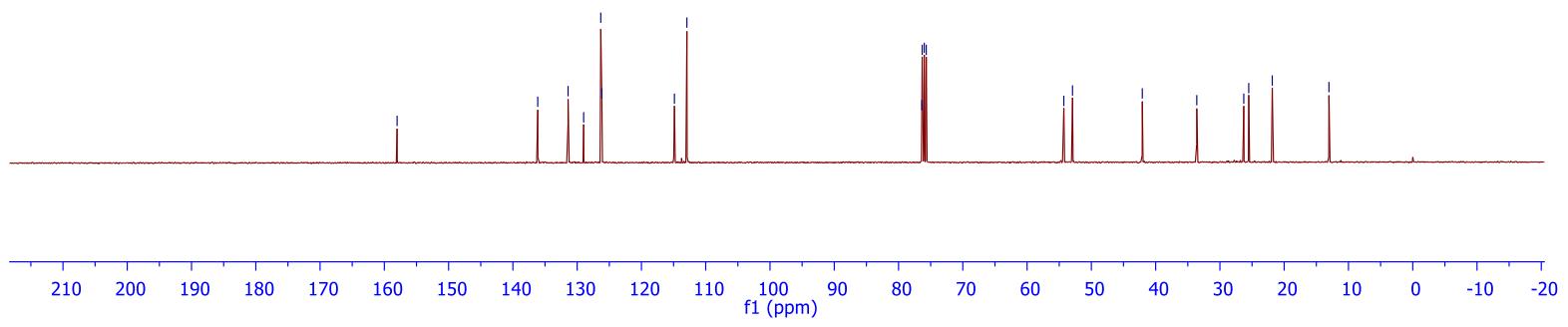

SI-50 


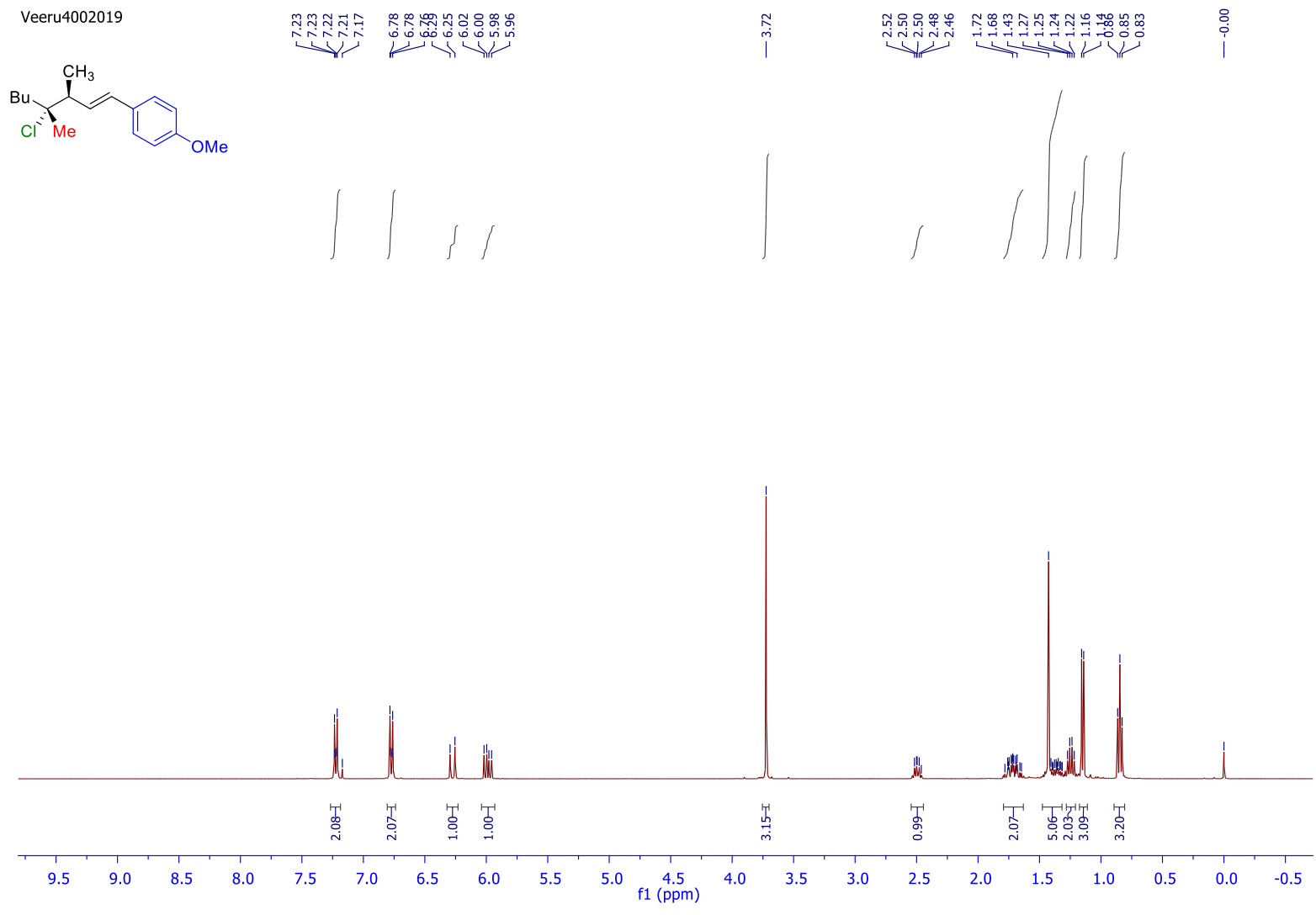

Veeru4002019

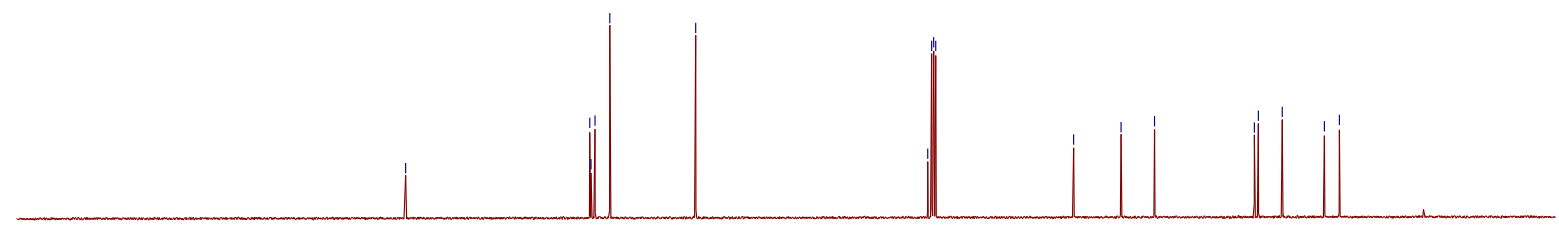

$\begin{array}{llllllllllllllllllllllll}210 & 200 & 190 & 180 & 170 & 160 & 150 & 140 & 130 & 120 & 110 & 100 & 100 & 80 & 70 & 60 & 50 & 40 & 30 & 20 & 10 & 0 & -10\end{array}$ 
Cl
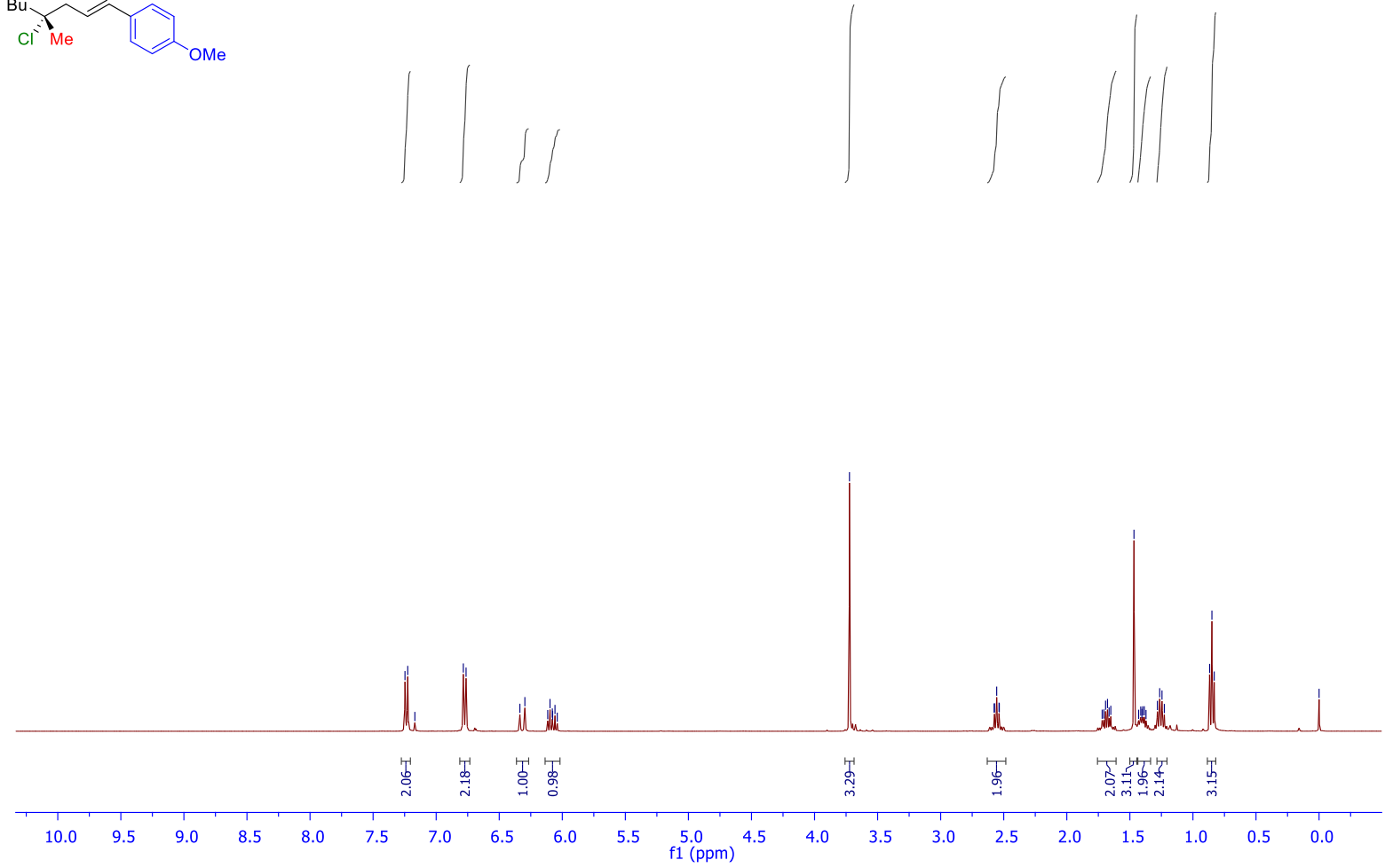

Veeru4002019

|

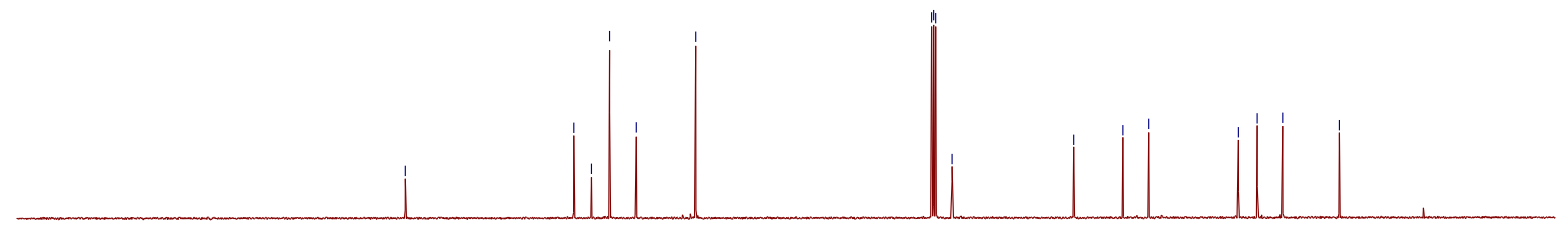

$\begin{array}{llllllllllllllllllllllll}210 & 200 & 190 & 180 & 170 & 160 & 150 & 140 & 130 & 120 & 110 & \begin{array}{c}100 \\ \mathrm{f} 1(\mathrm{ppm})\end{array} & 90 & 80 & 70 & 60 & 50 & 40 & 30 & 20 & 10 & 0 & -10 & -20\end{array}$ 
Veeru400

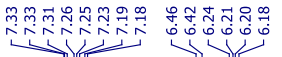

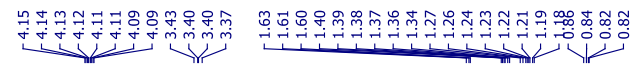
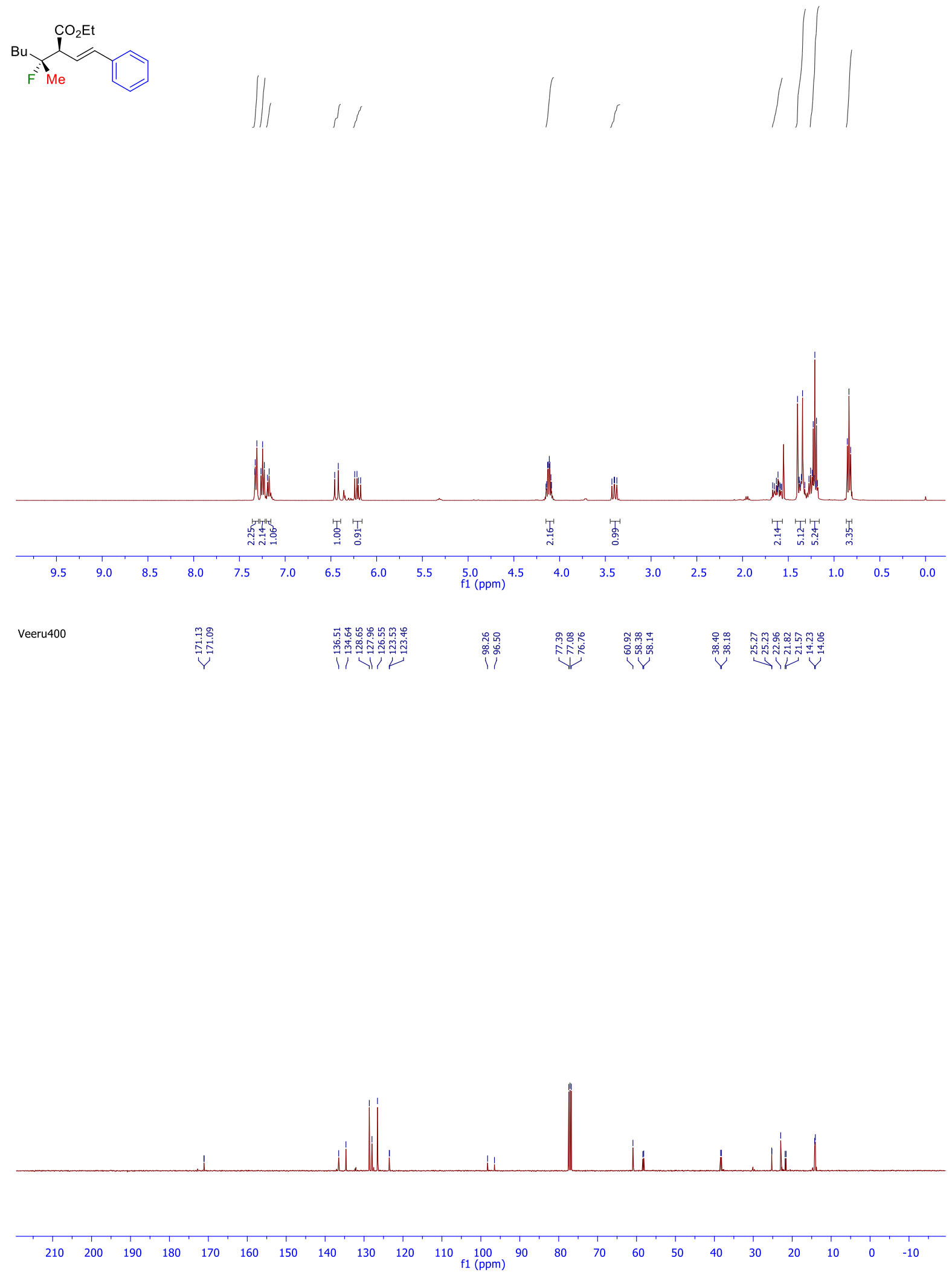

SI-53 
Veeru4002020

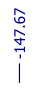
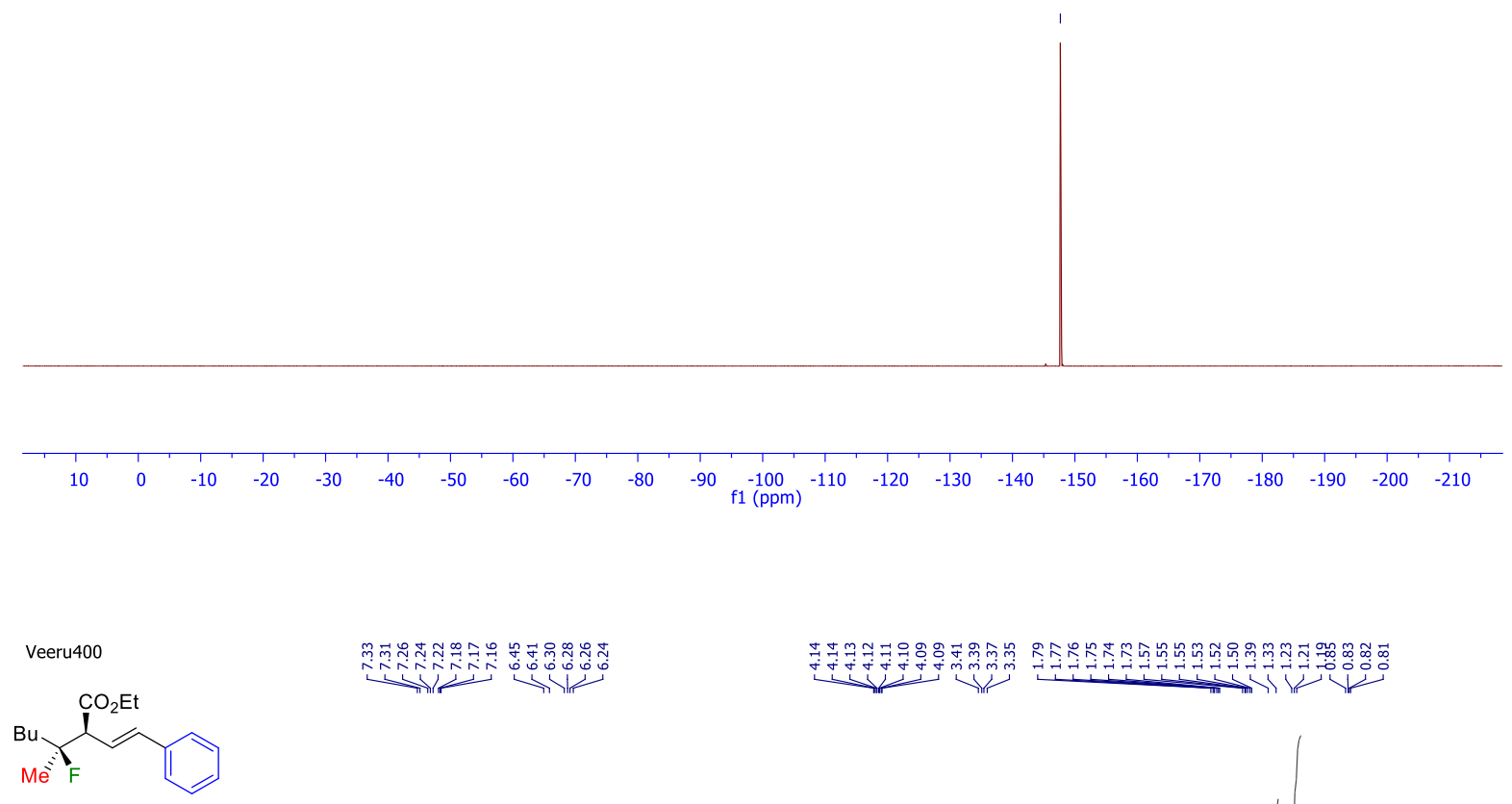

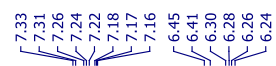

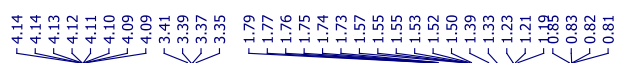
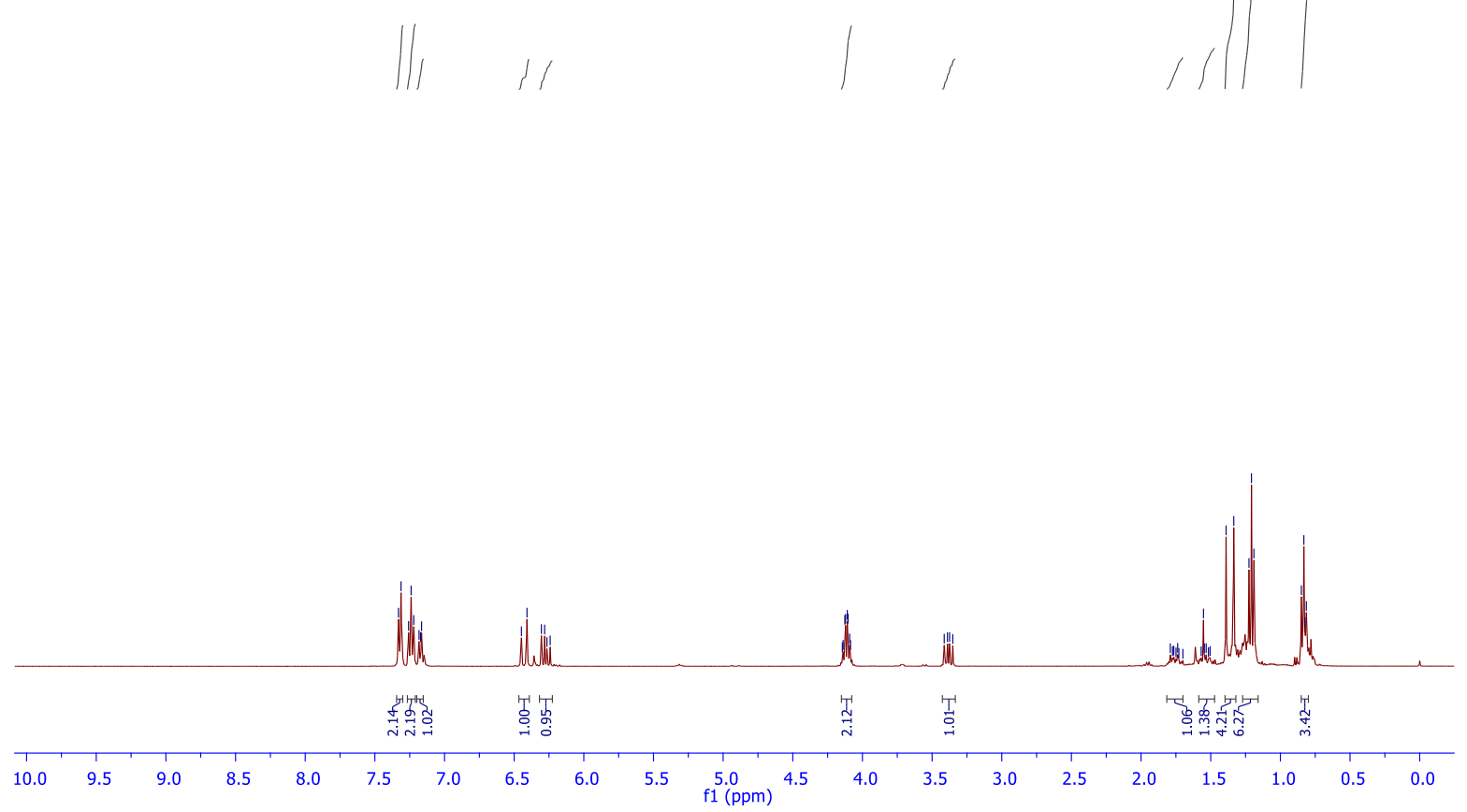

SI-54 
Veeru400

\begin{tabular}{|c|c|c|c|c|c|}
\hline 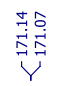 & 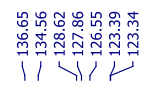 & 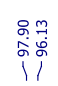 & 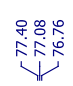 & 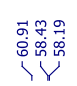 & $\underbrace{\substack{m \\
m}}$ \\
\hline
\end{tabular}

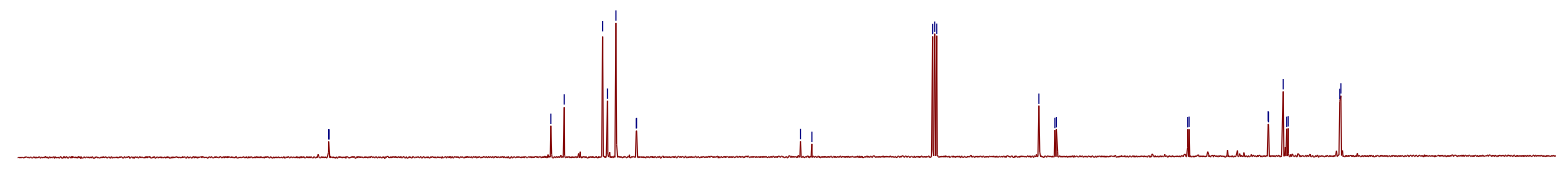

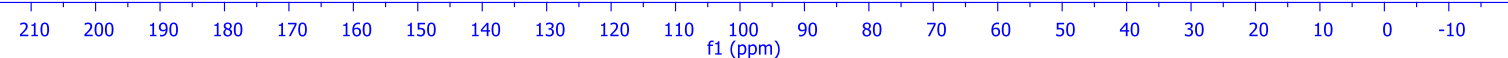

Veeru400

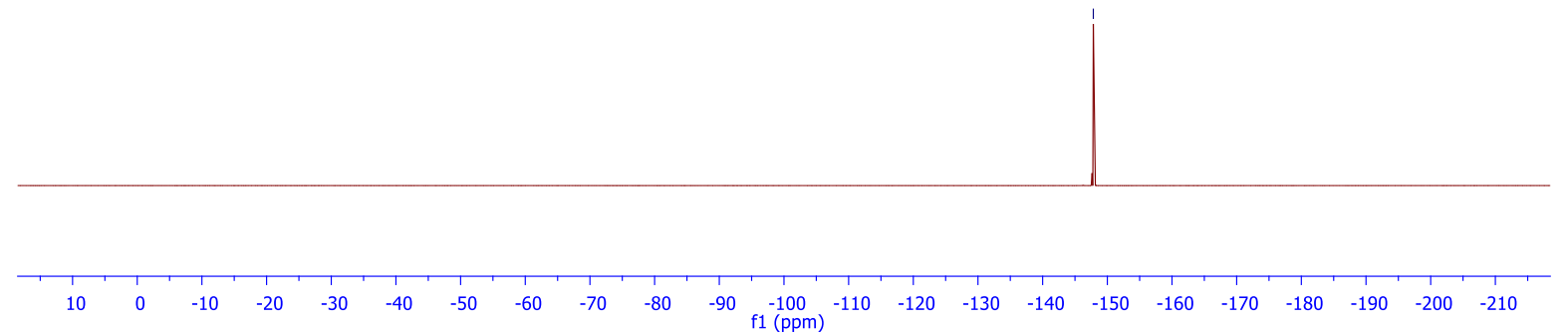


<smiles>CCOC(=O)C(C=Cc1cccc2ccccc12)C(C)([NH3+])CBr</smiles><smiles>C=CC1=CCCC=C1</smiles><smiles>CC=CC</smiles>

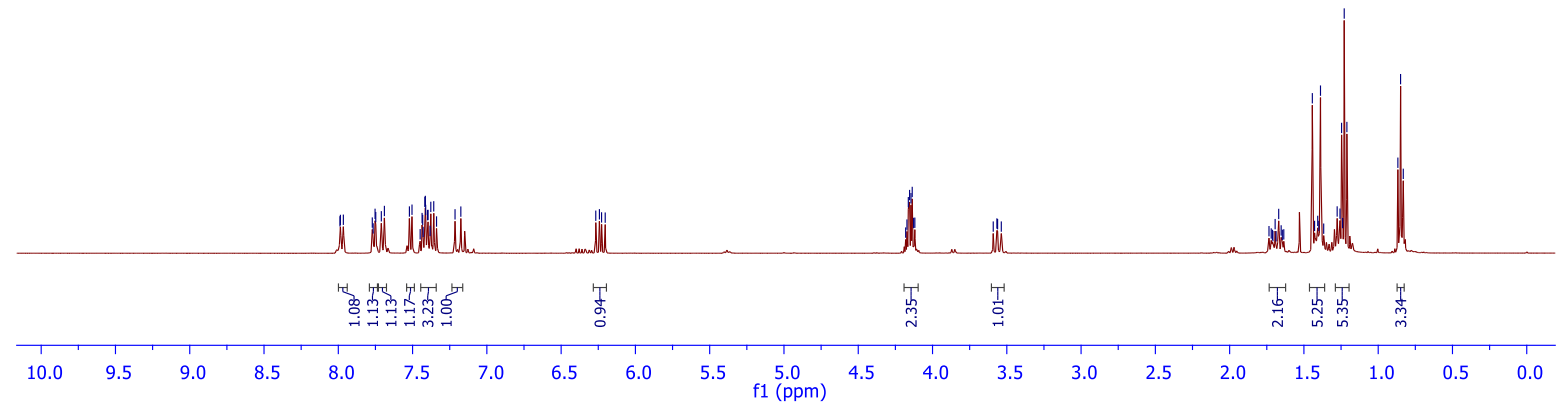

Veeru4002019

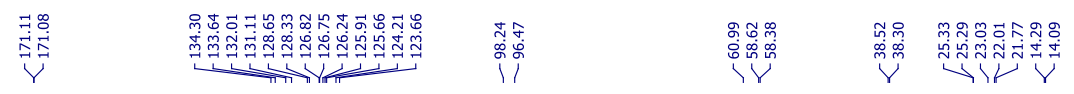

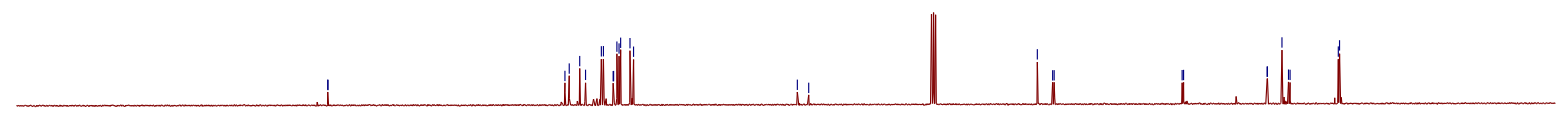

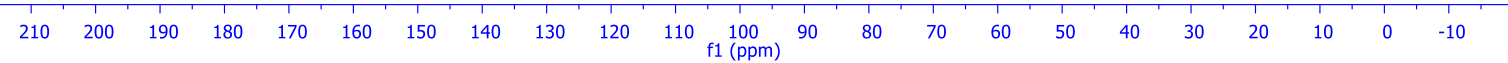


Veeru4002019

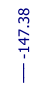
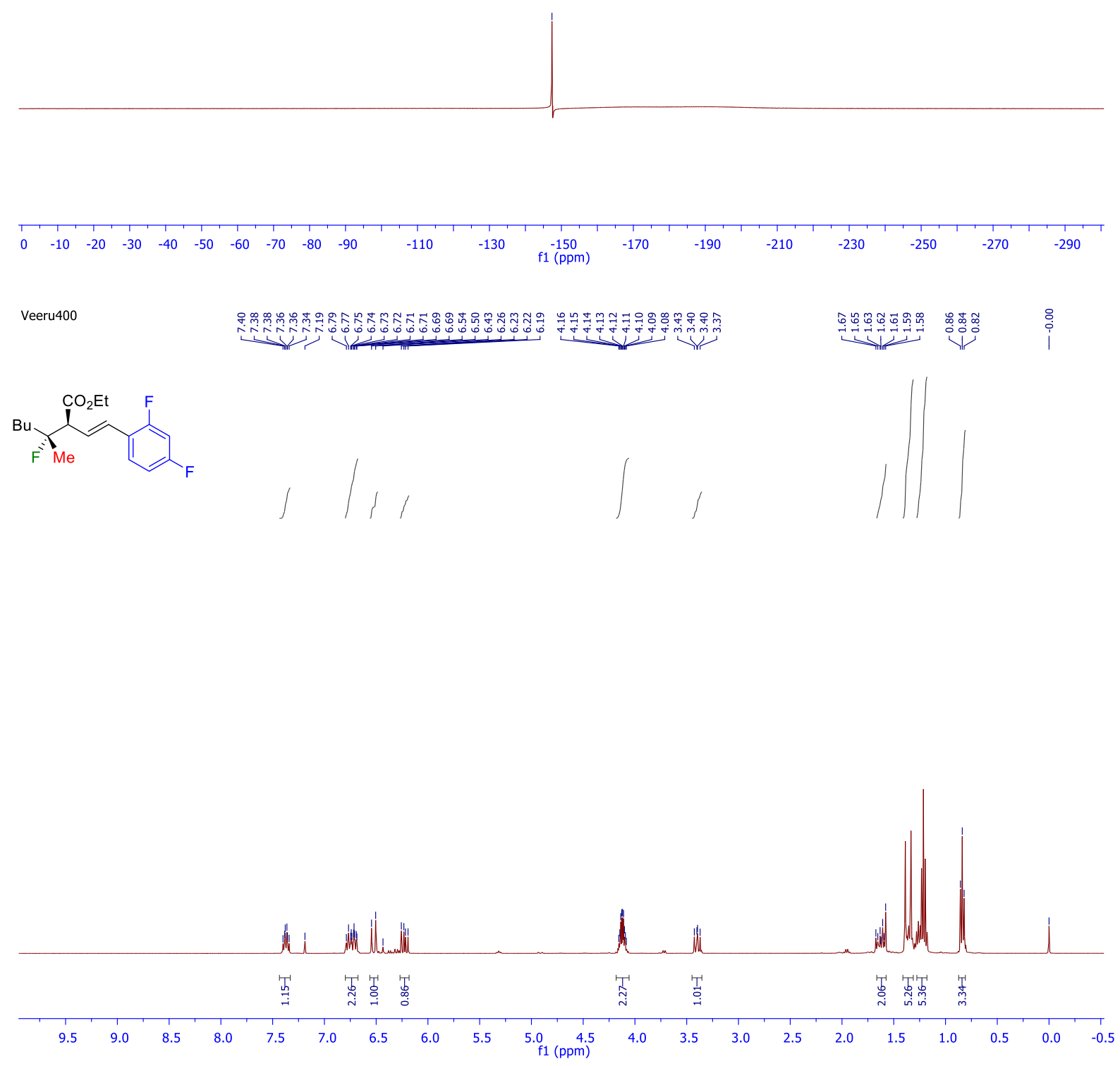

SI-57 
Veeru400

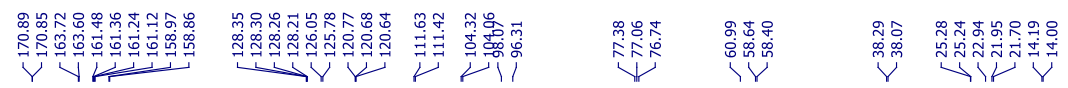

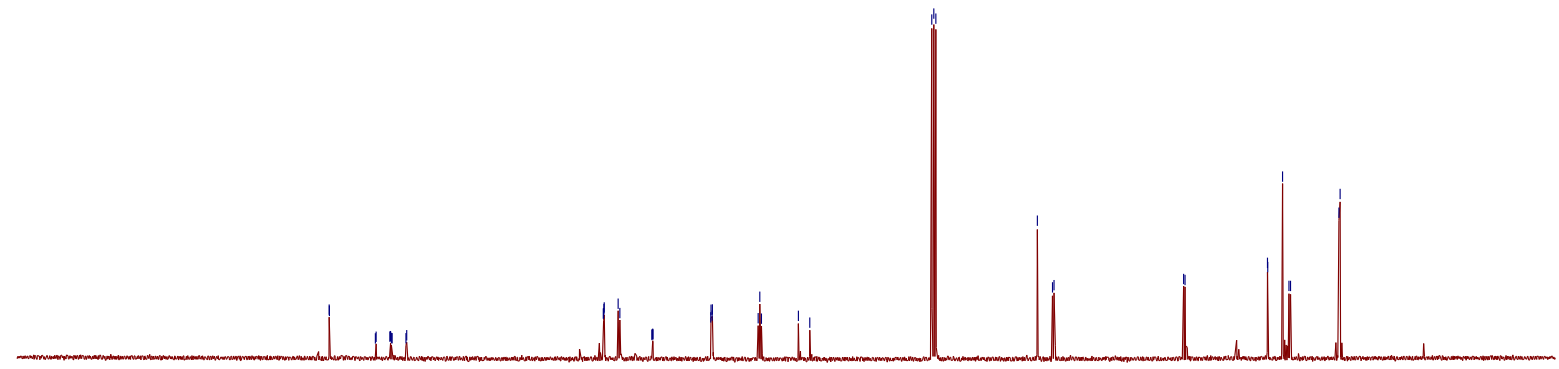

$\begin{array}{lllllllllllllllllllllll}210 & 200 & 190 & 180 & 170 & 160 & 150 & 140 & 130 & 120 & 110 & \underset{\mathrm{f} 1(\mathrm{ppm})}{100} & 90 & 80 & 70 & 60 & 50 & 40 & 30 & 20 & 10 & 0 & -10\end{array}$

Veeru400

ivi

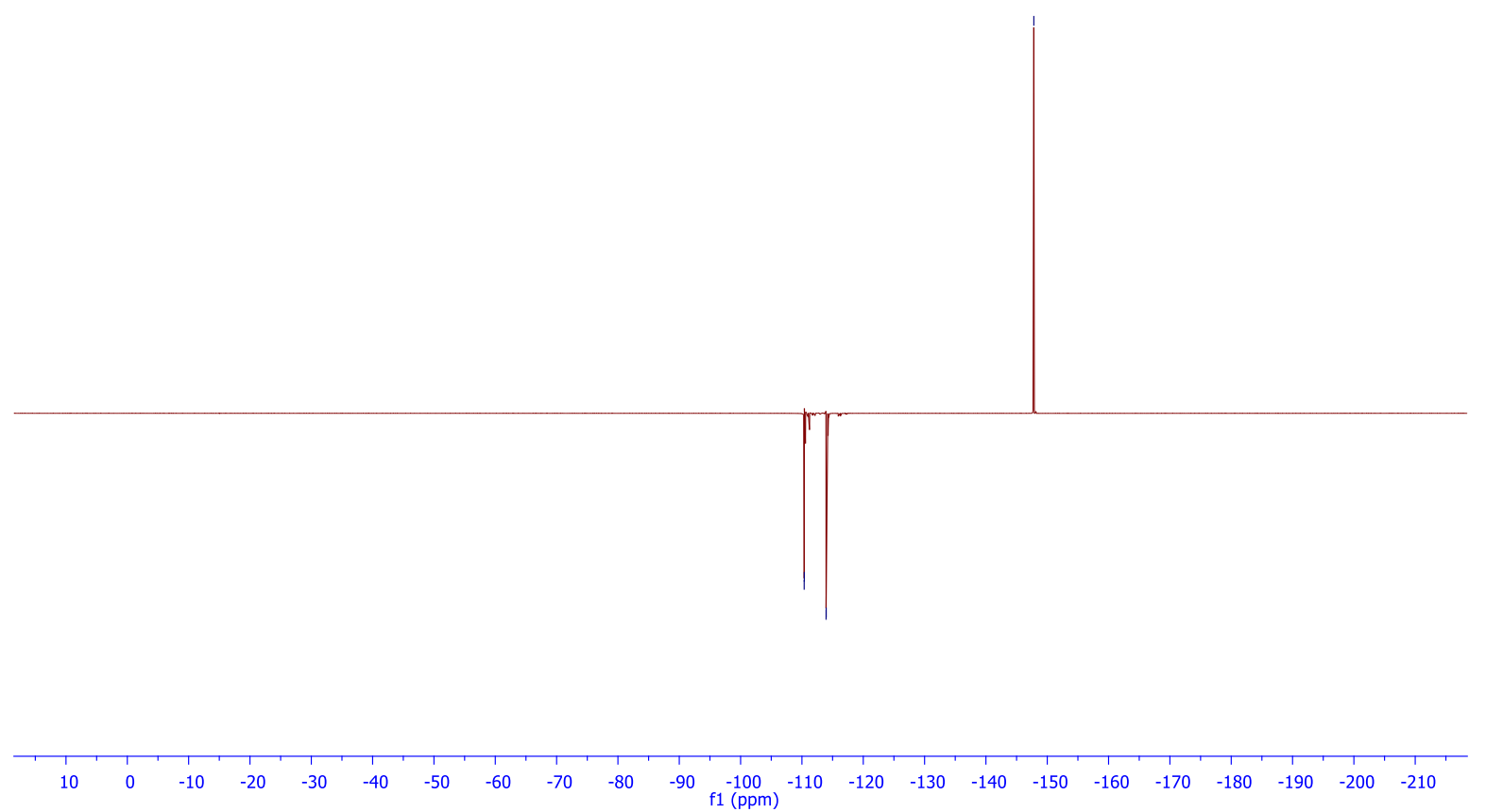


Veeru400

(1)
ำ

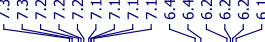

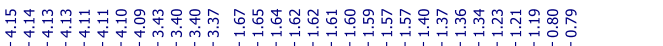

作

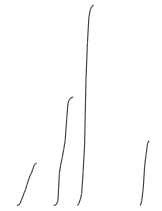

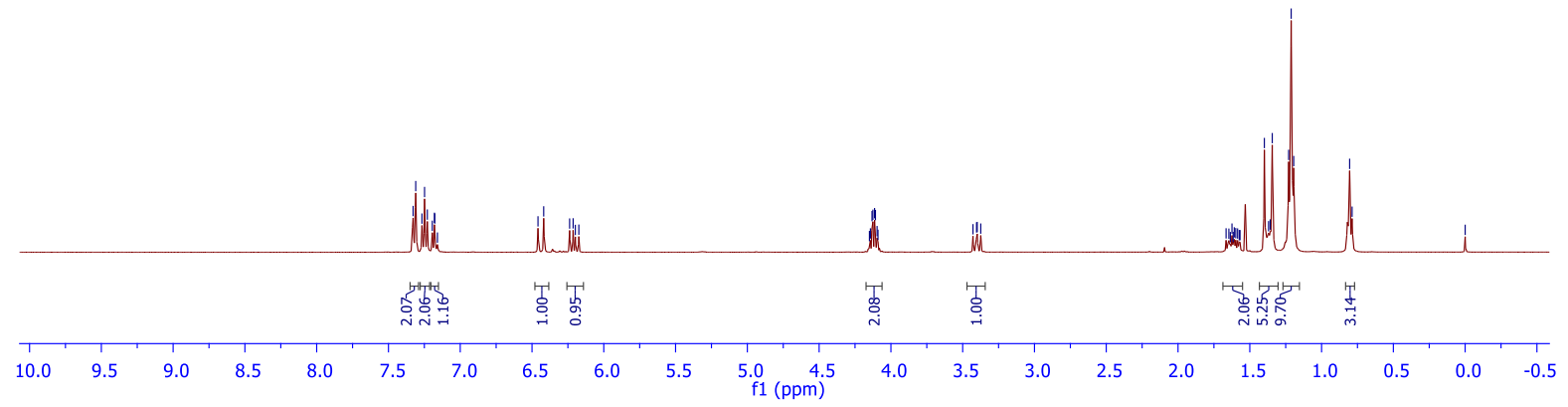

Veeru400

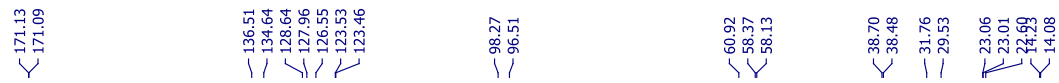

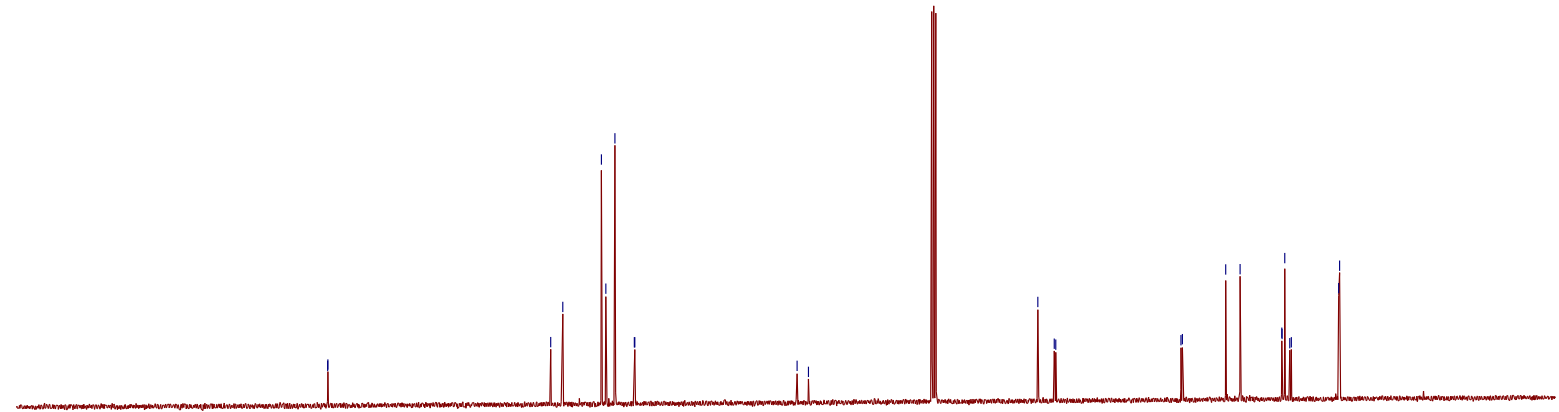

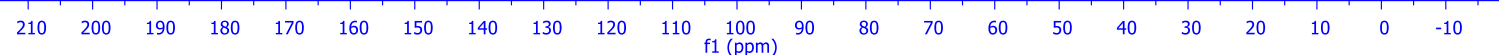


$\operatorname{Vann11nn}_{1_{10}} \operatorname{NMR}\left(377 \mathrm{MHz}, \mathrm{CDCl}_{3}\right) \delta-147.45$.

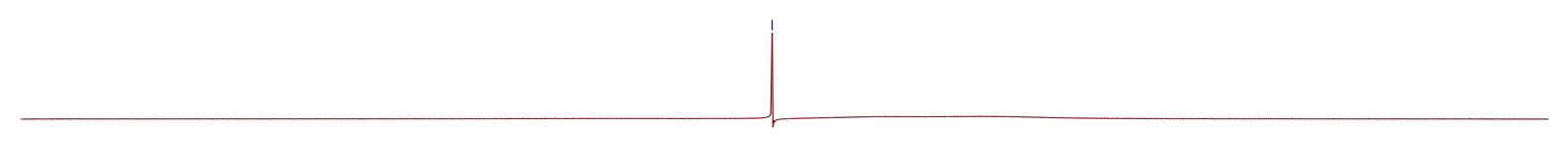

\begin{tabular}{llllllllllllllllllllll}
\hline \\
0 & -10 & -20 & -30 & -40 & -50 & -60 & -70 & -80 & -90 & -110 & -130 & -150 & -170 & -190 & -210 & -230 & -250 & -270 & -290
\end{tabular}
$\mathrm{BnO}\left(\mathrm{CH}_{2}\right)_{2}$

Veeru400

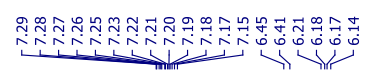

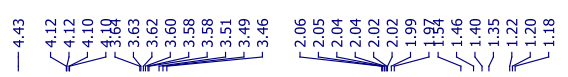
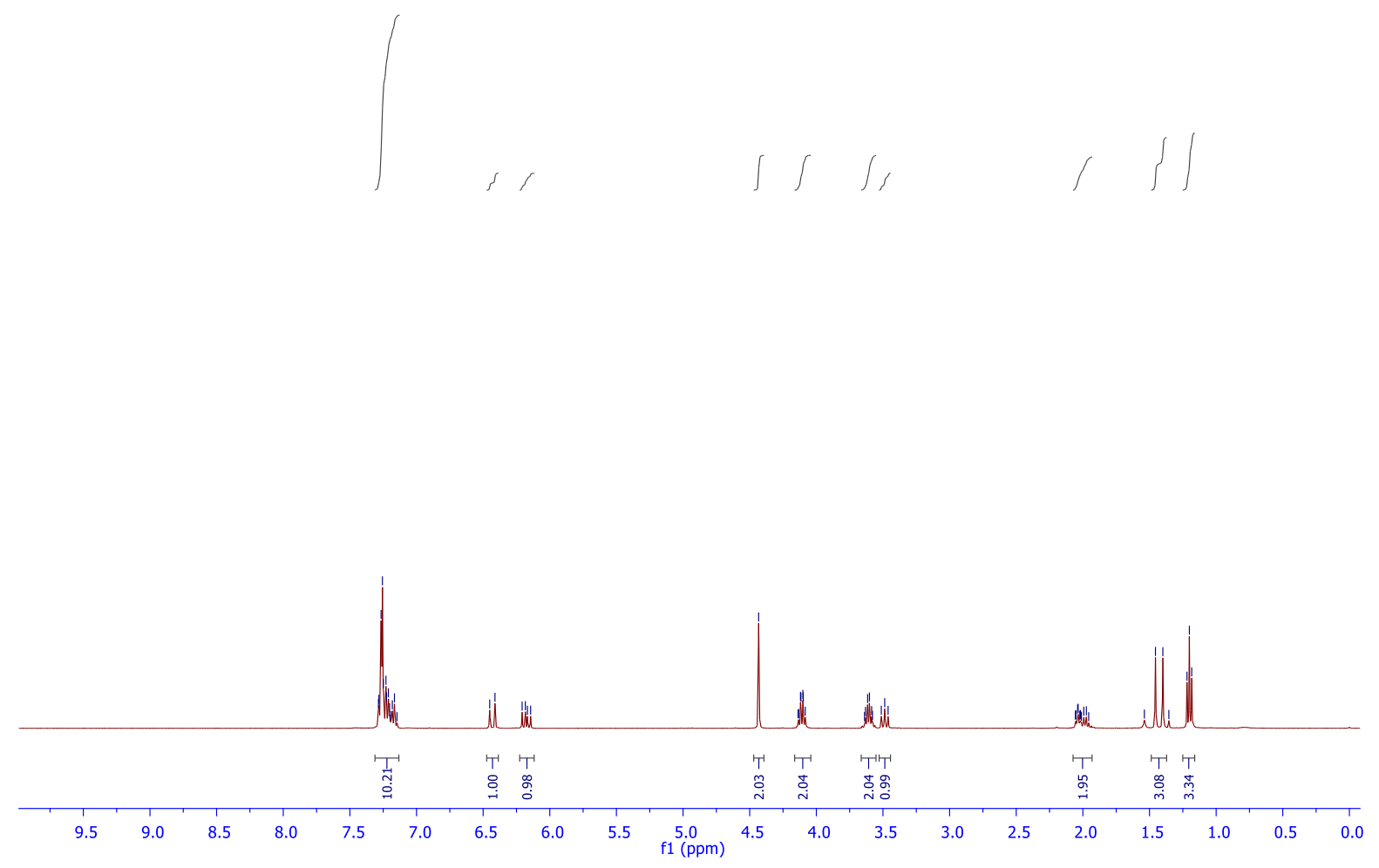
Veeru400

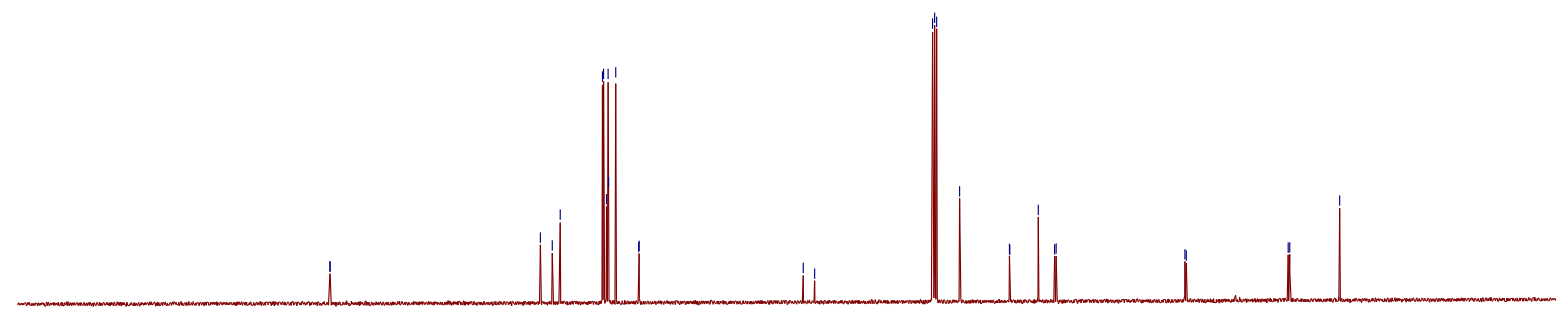

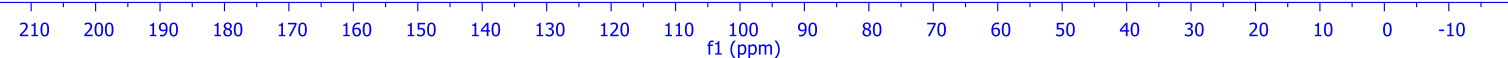

Veeru400

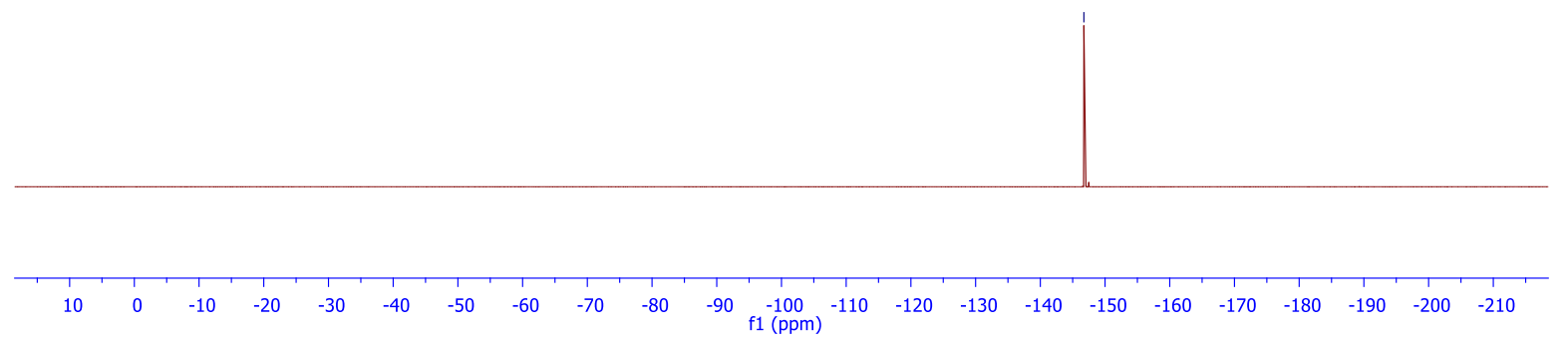


(ne
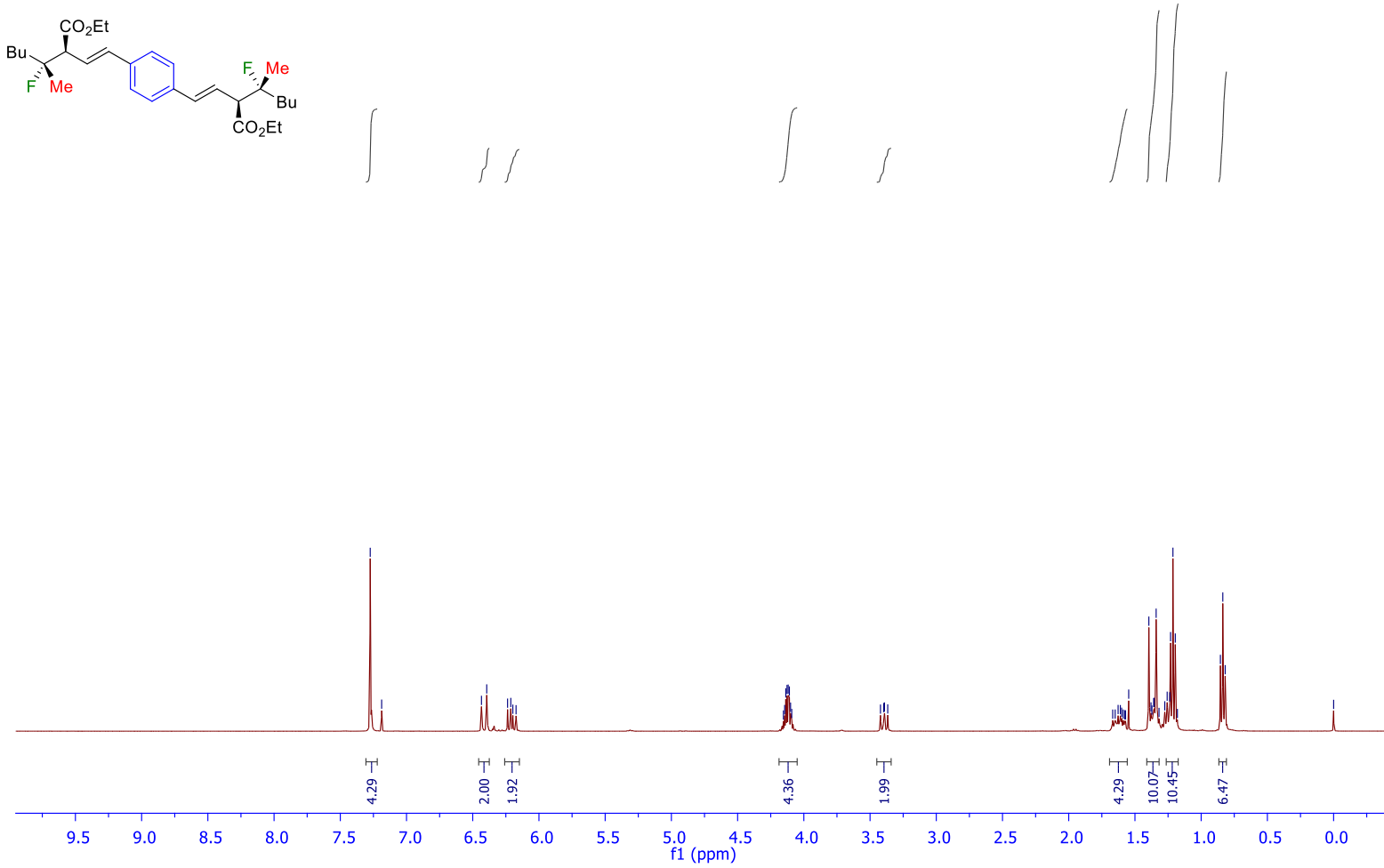

Veeru4002019

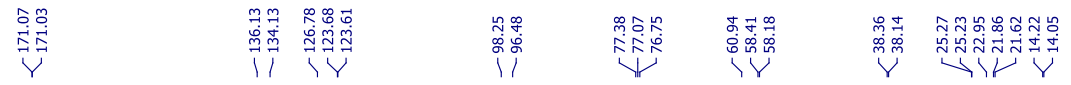

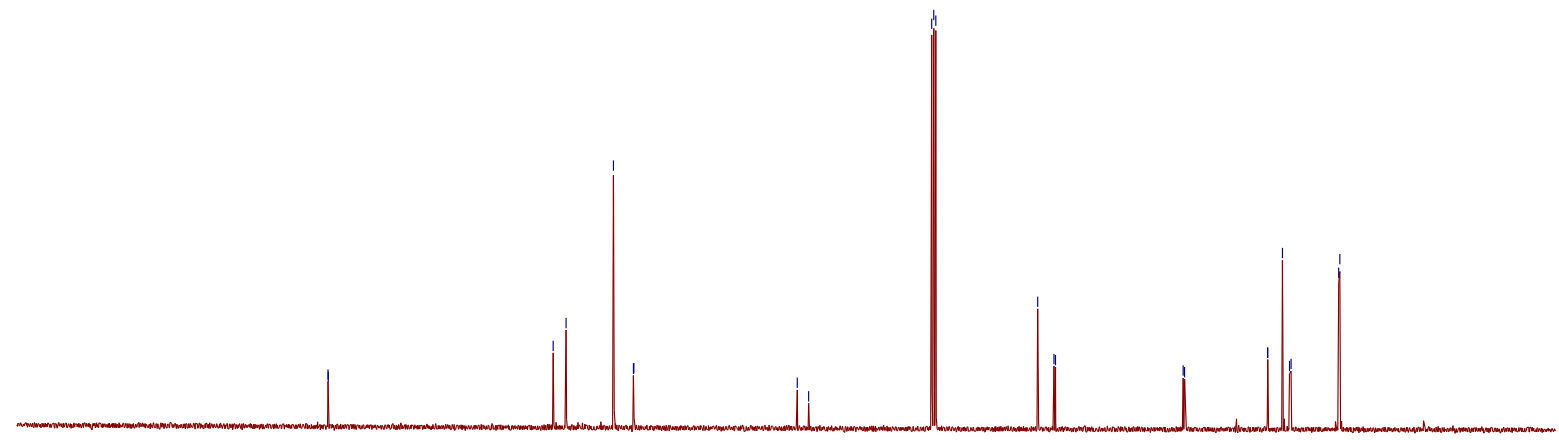

$\begin{array}{llllllllllllllllllllllllllll}210 & 200 & 190 & 180 & 170 & 160 & 150 & 140 & 130 & 120 & 110 & \underset{f}{100} & 90 & 80 & 70 & 60 & 50 & 40 & 30 & 20 & 10 & 0 & -10\end{array}$ 
Veeru 4002020

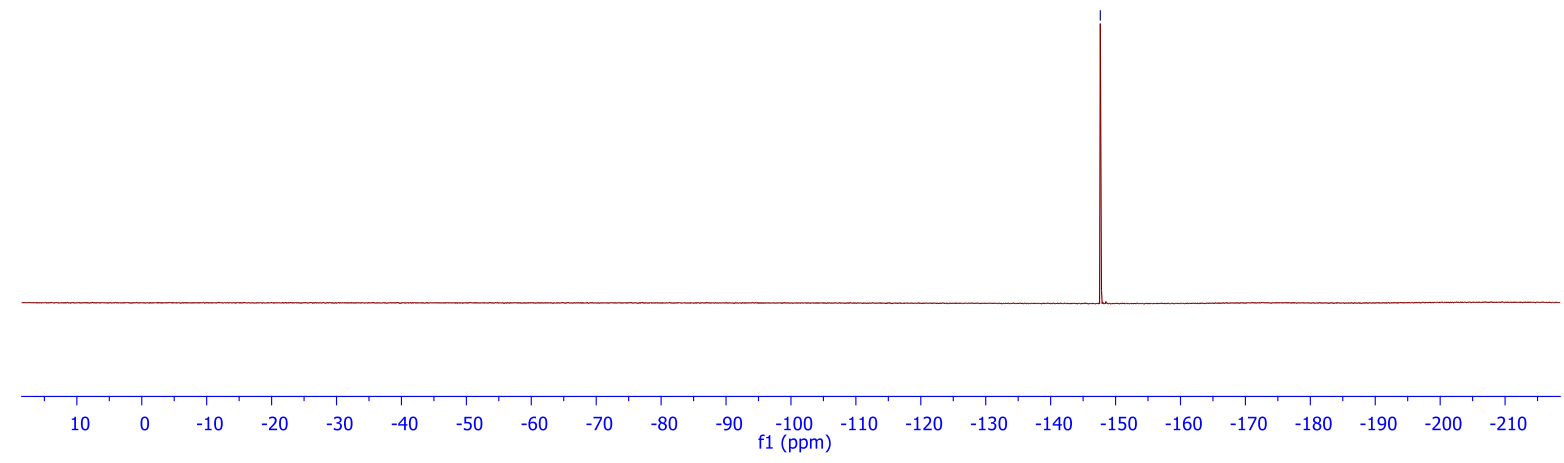

Veeru400

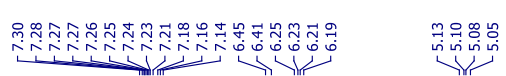

$\underbrace{\text { ming }}$

(1)

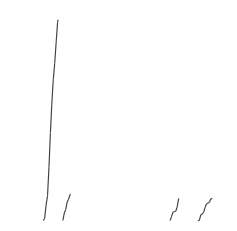

$\int$

l
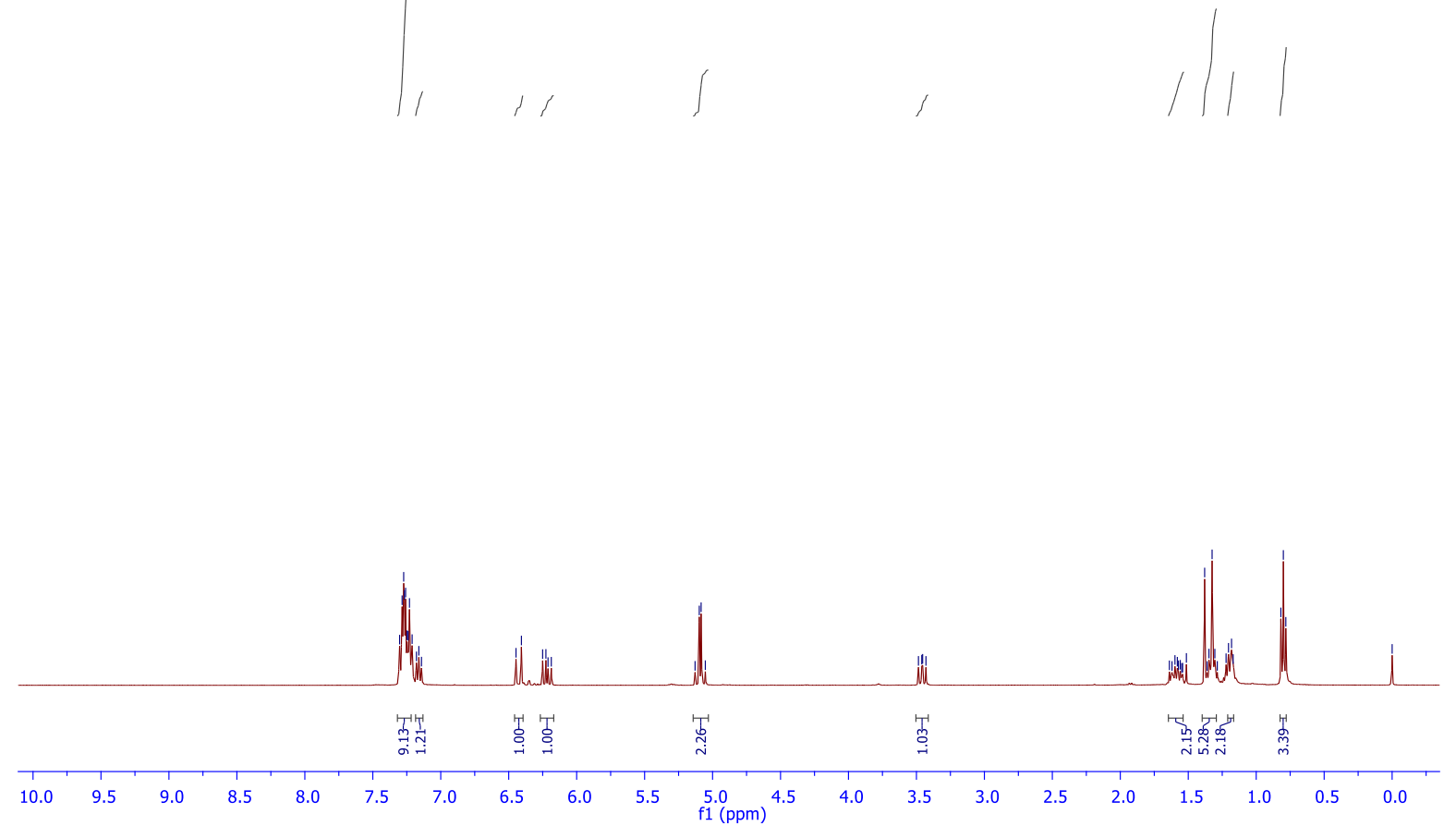
Veeru400

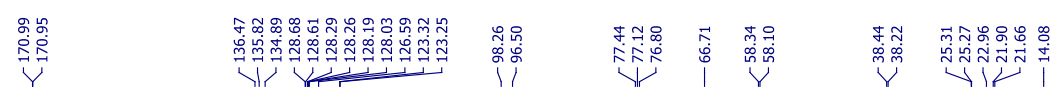

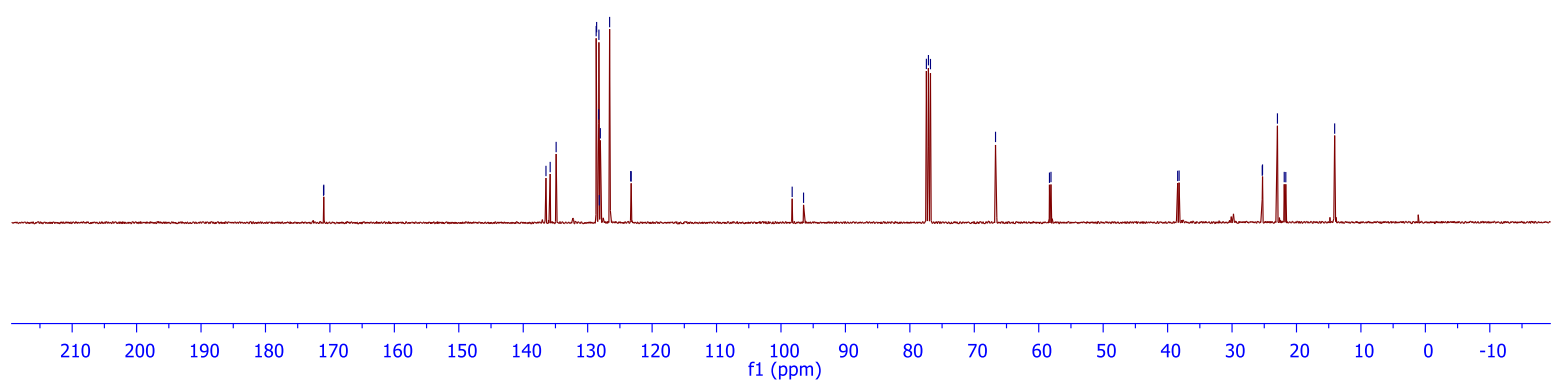

Veeru400

$\stackrel{\substack{n \\ \stackrel{n}{i}}}{i}$

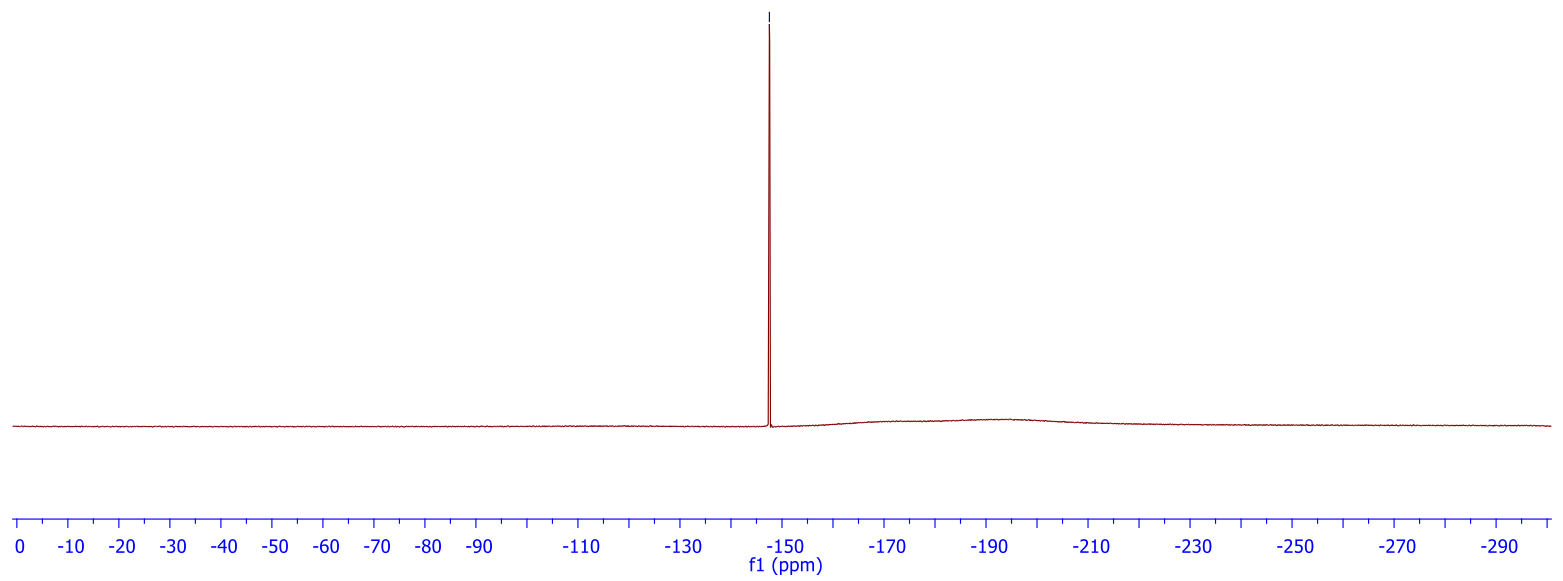

SI-64 
<smiles>C=CCC(C=Cc1ccc(OC)cc1)C(=O)Br</smiles>

$65 \%$ along with an inseparable mixture of $18 \%$ (out of $65 \%$ ) eliminated product

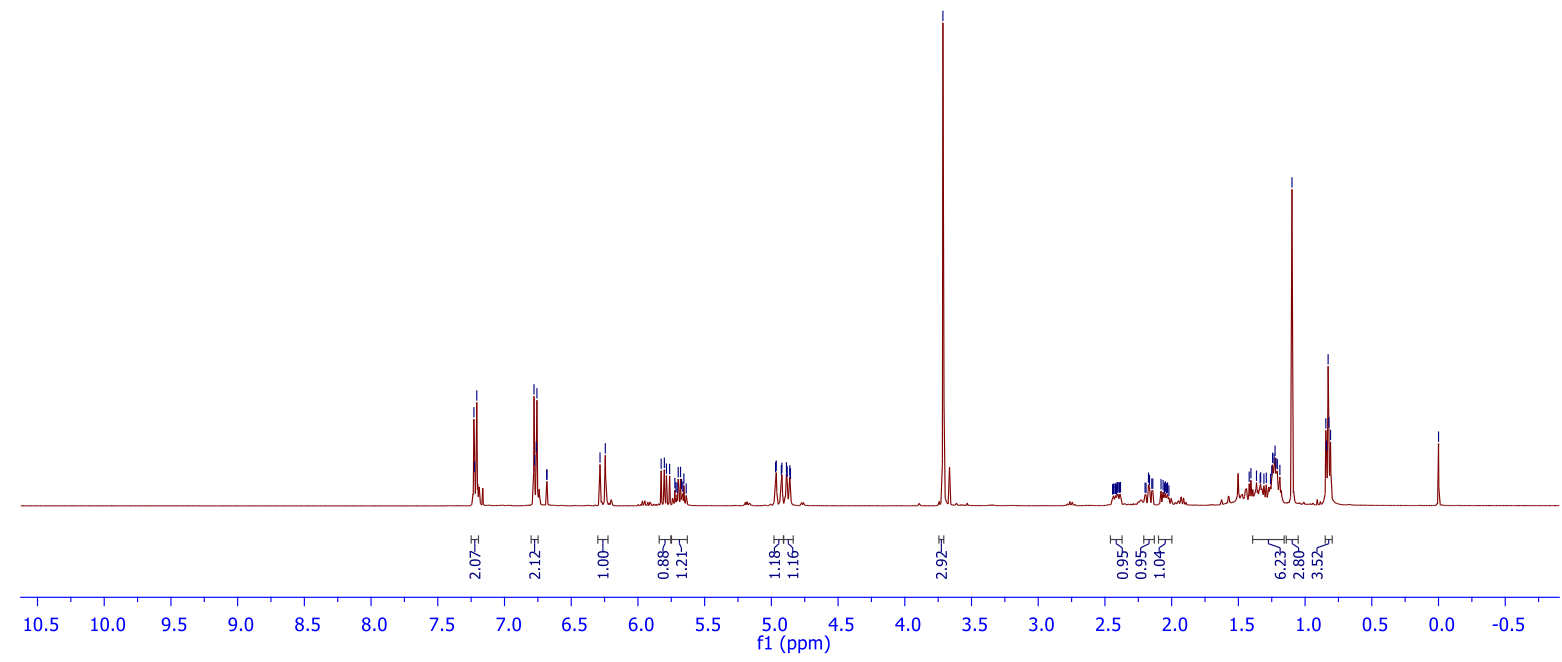

Veeru4002020

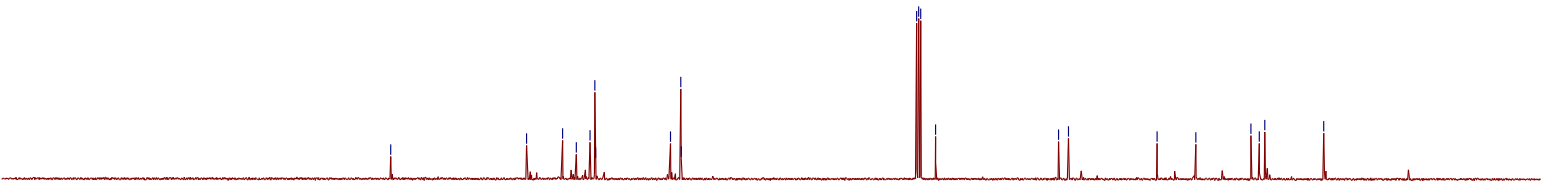

$\begin{array}{llllllllllllllllllllllll}210 & 200 & 190 & 180 & 170 & 160 & 150 & 140 & 130 & 120 & 110 & \begin{array}{c}100 \\ \mathrm{f} 1(\mathrm{ppm})\end{array} & 90 & 80 & 70 & 60 & 50 & 40 & 30 & 20 & 10 & 0 & -10 & -20\end{array}$ 
Veeru 4002020
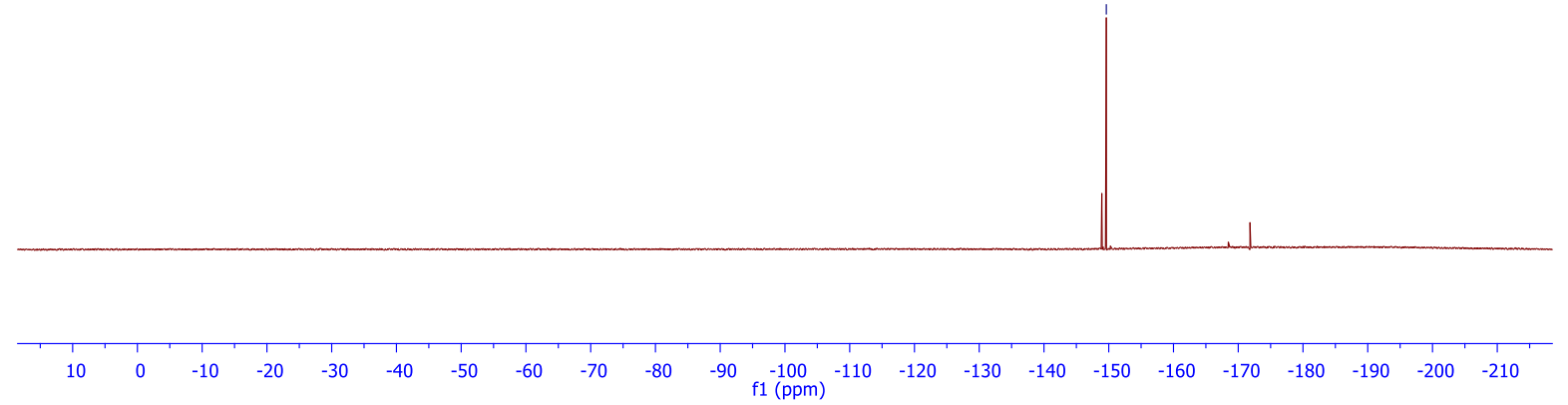

Veeru400

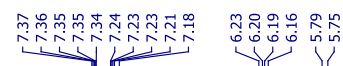

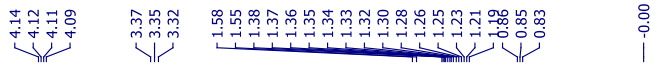<smiles>[M]C(F)(C(/C=C/C#Cc1ccccc1)C(=O)OCC)C(C)(C)C</smiles><smiles>C1=CCC=C1</smiles>
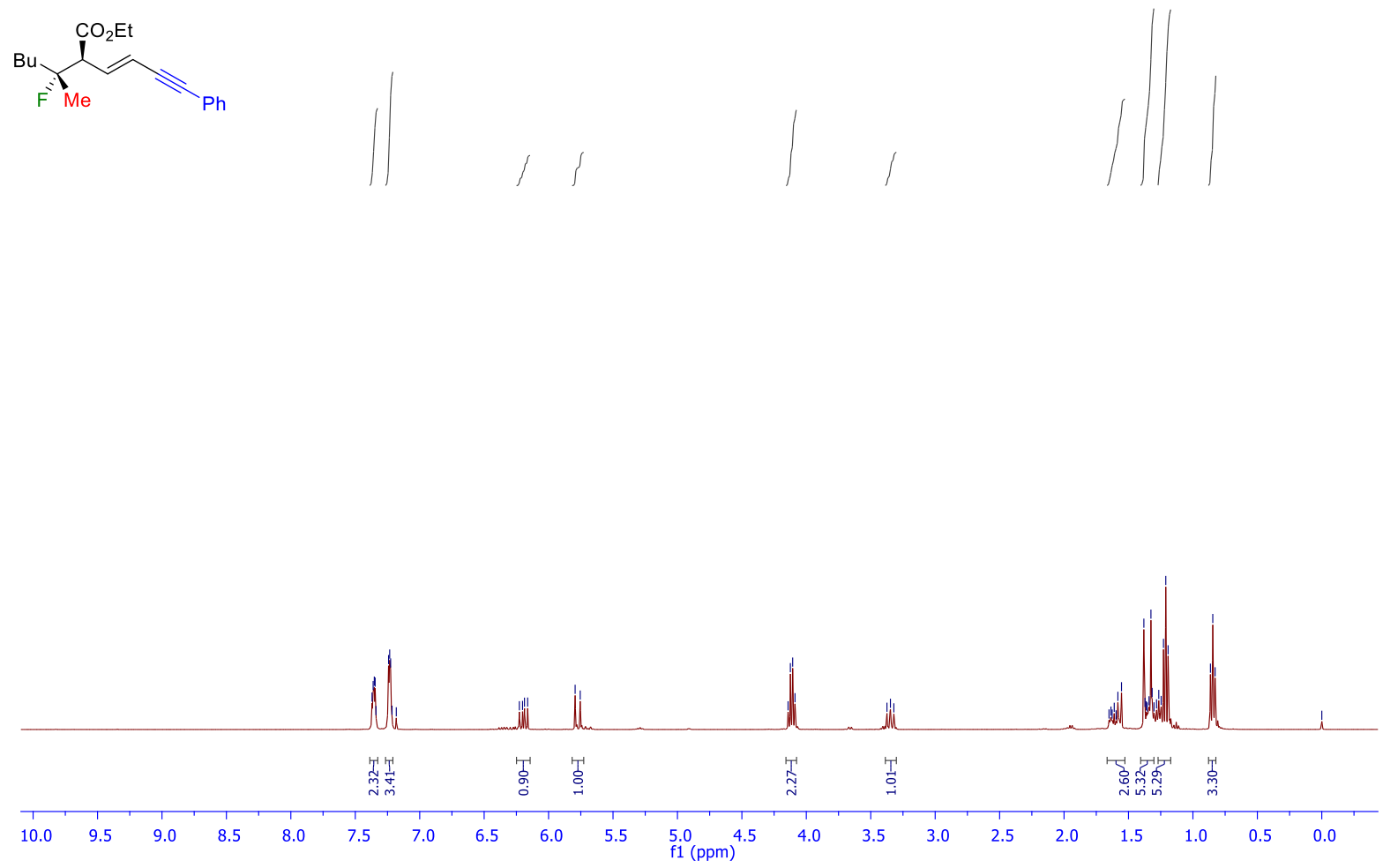
Veeru400 Yे

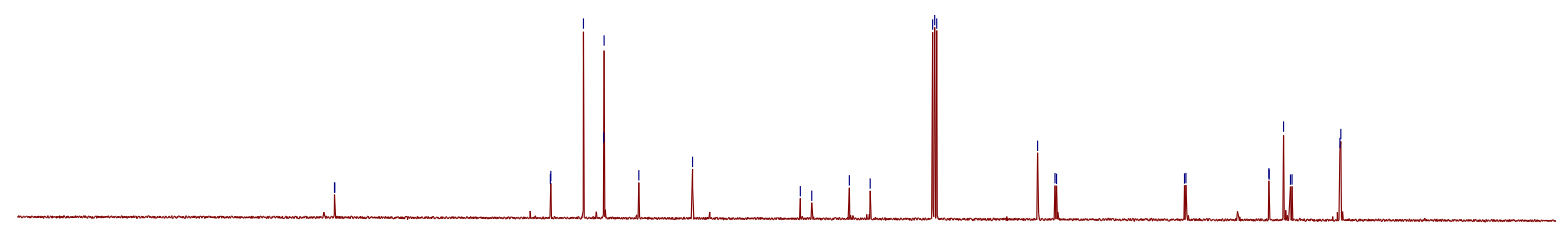

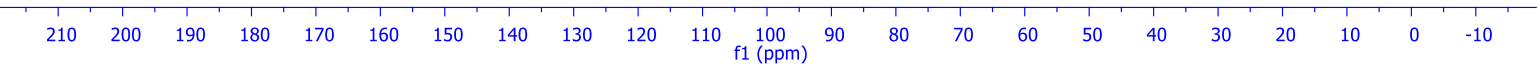

Veeru400

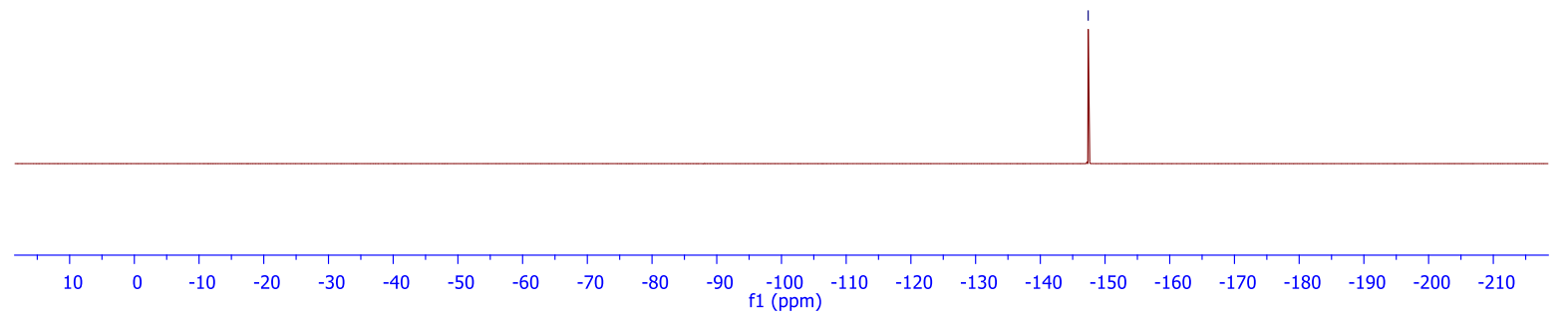




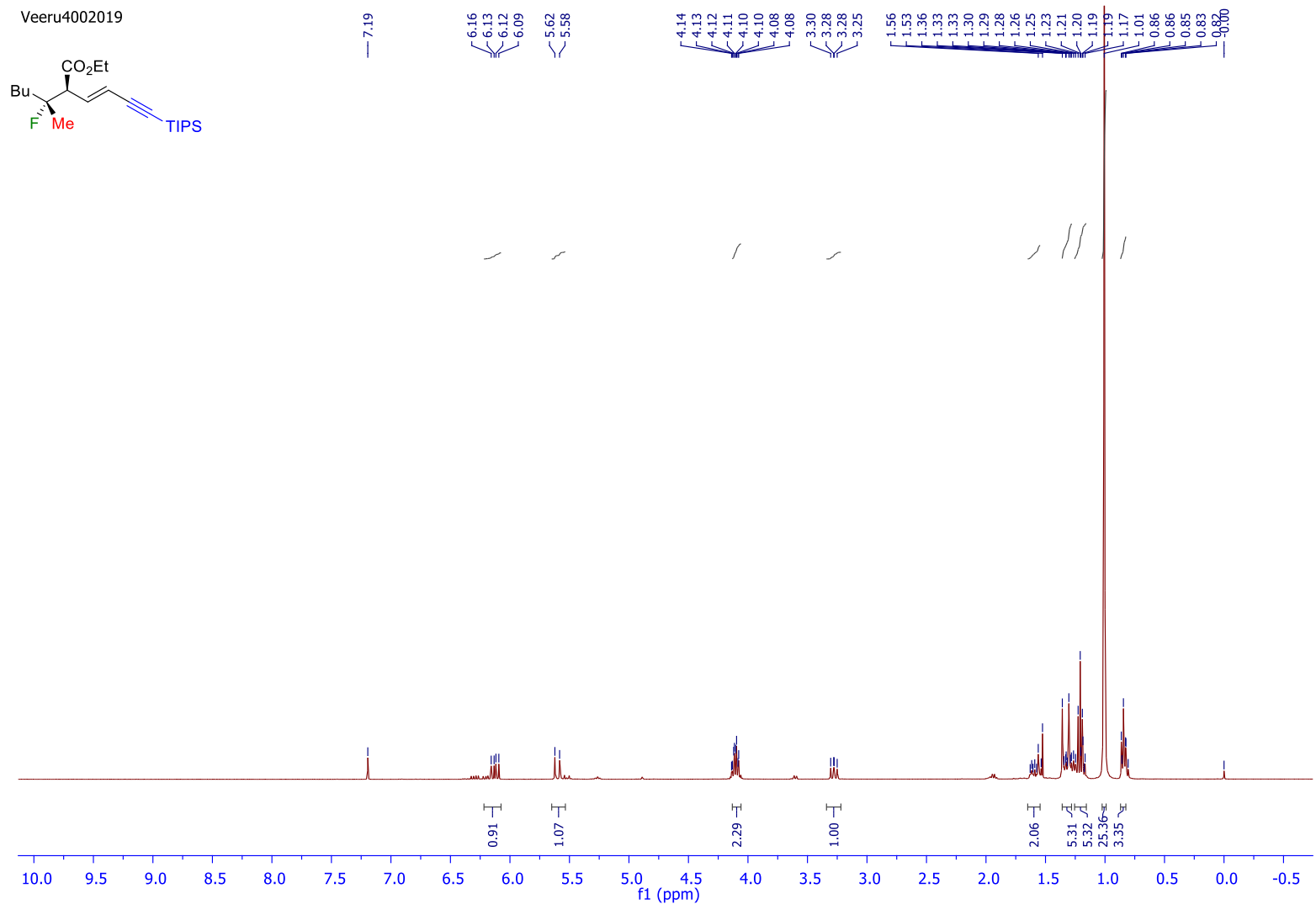

Veeru4002019

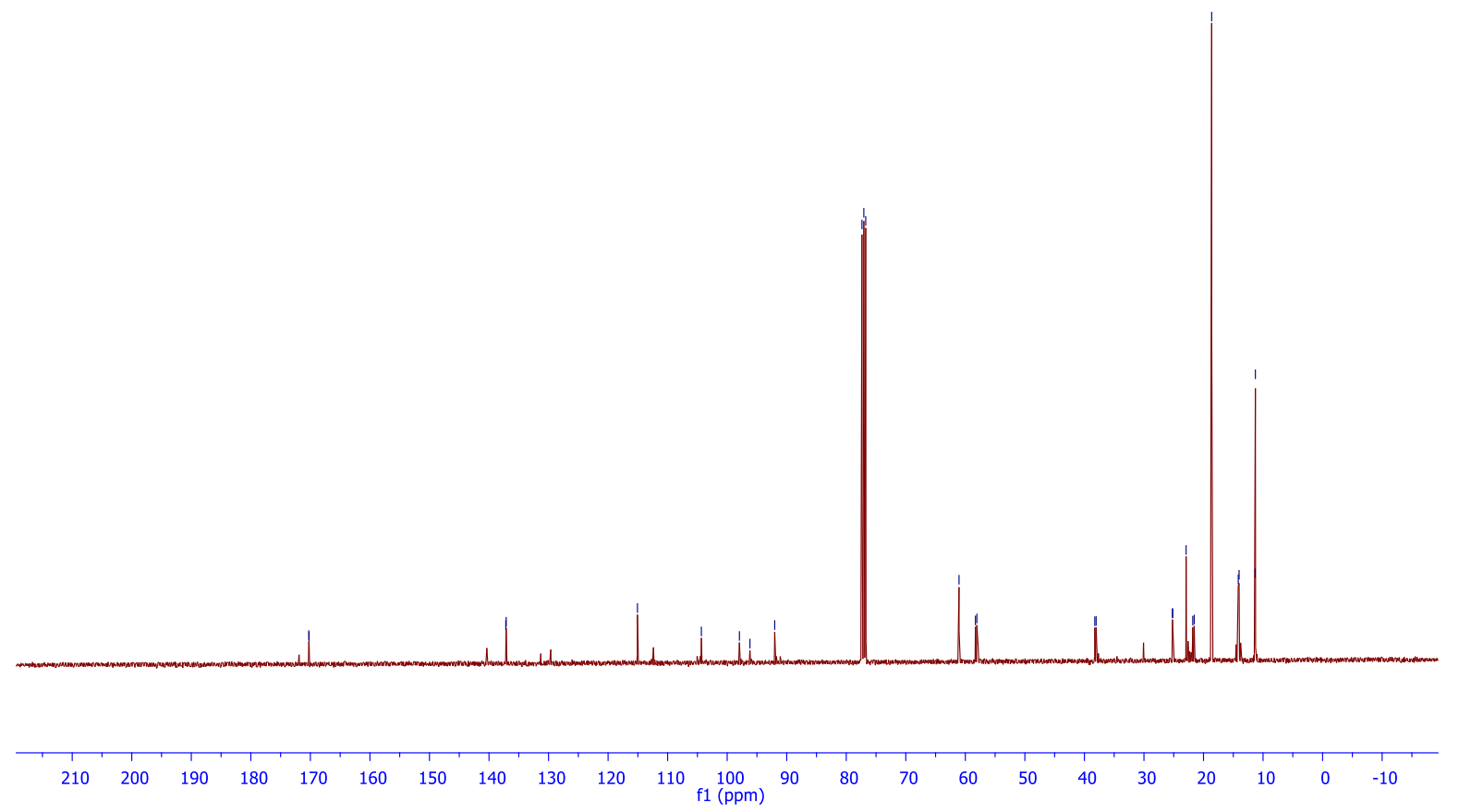


Veeru4002019

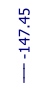
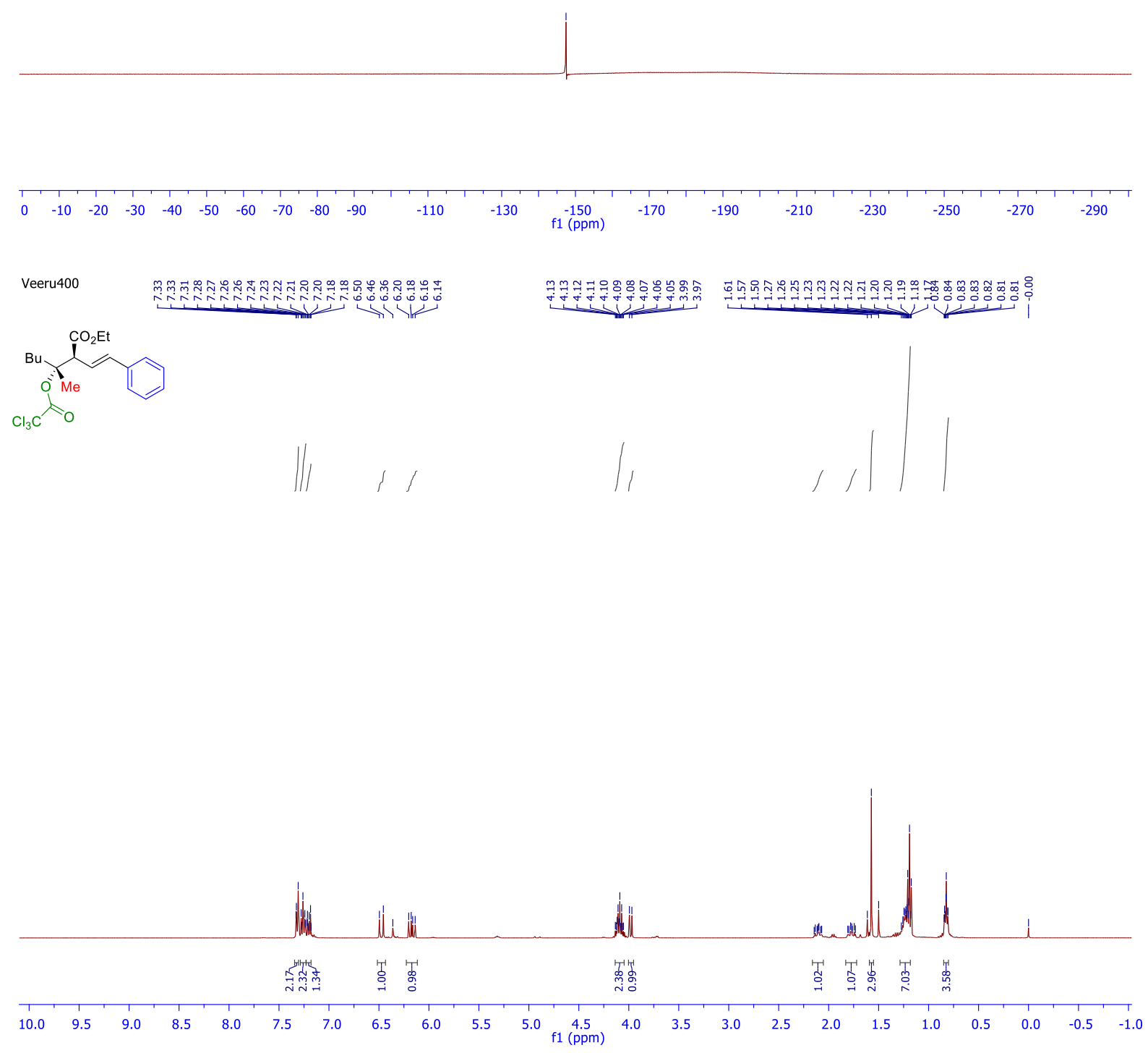

SI-69 
Veeru400

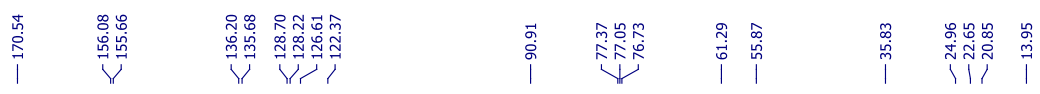

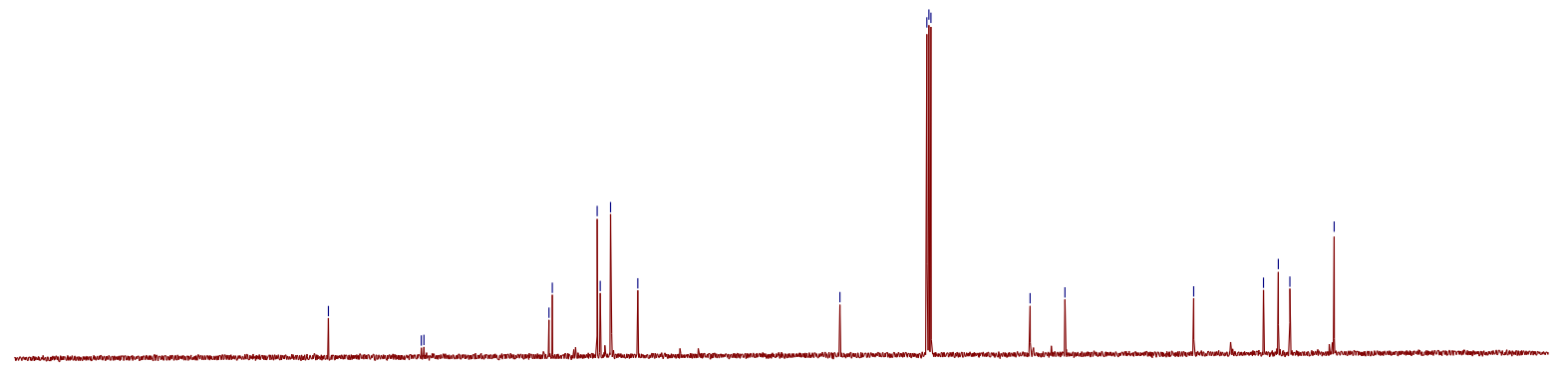

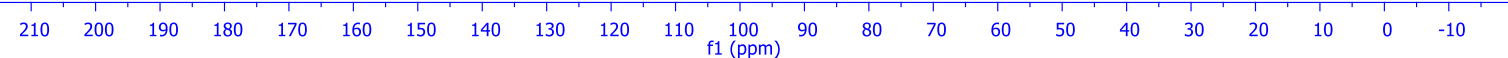

Veeru4002020 $\mathrm{Cl}_{3} \mathrm{O}_{\mathrm{Br}}$

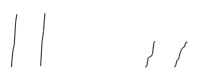

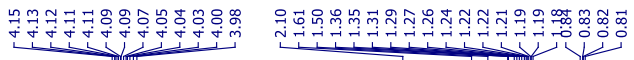
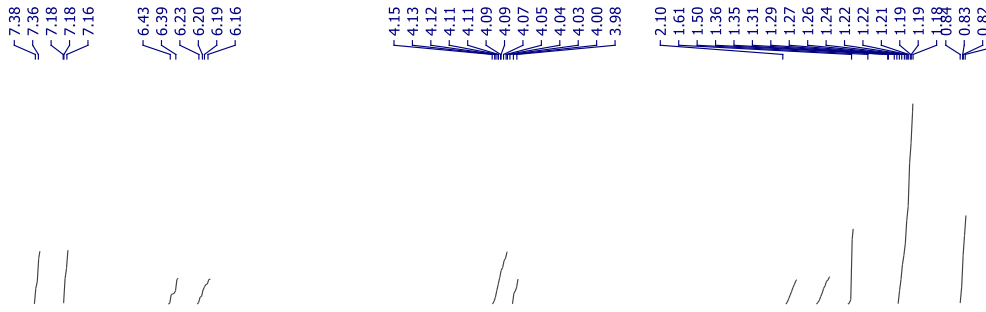

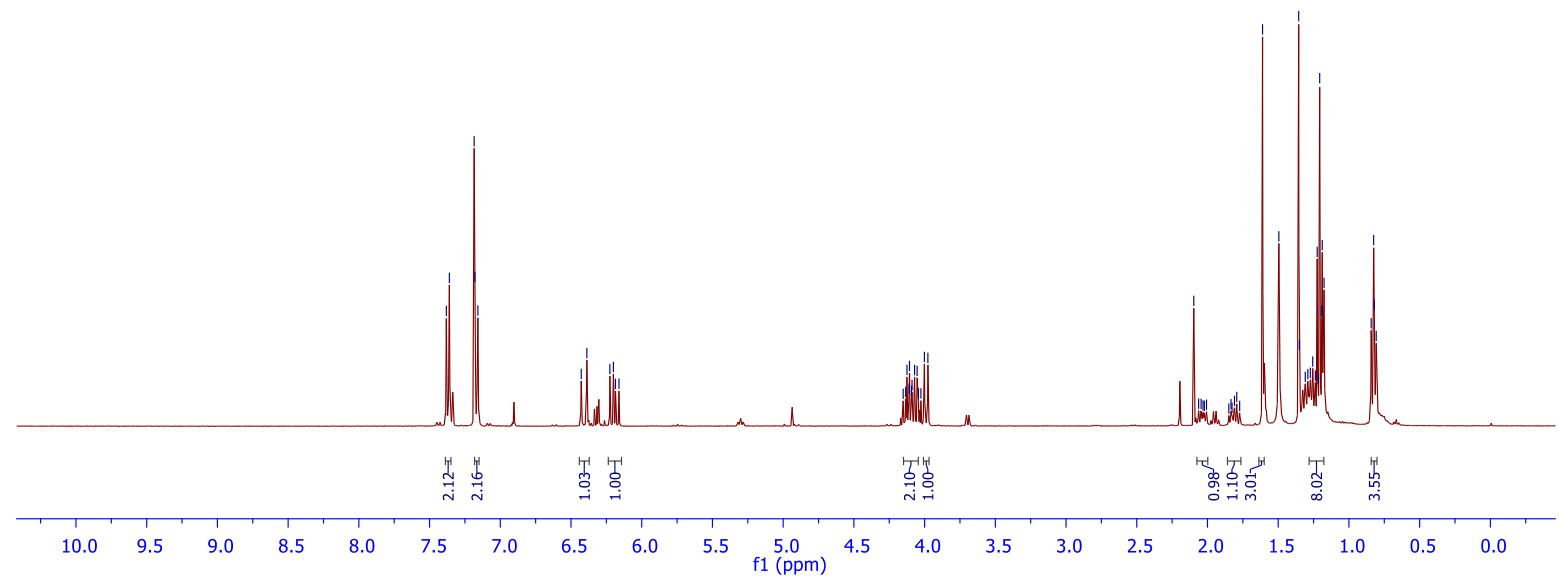


Veeru400

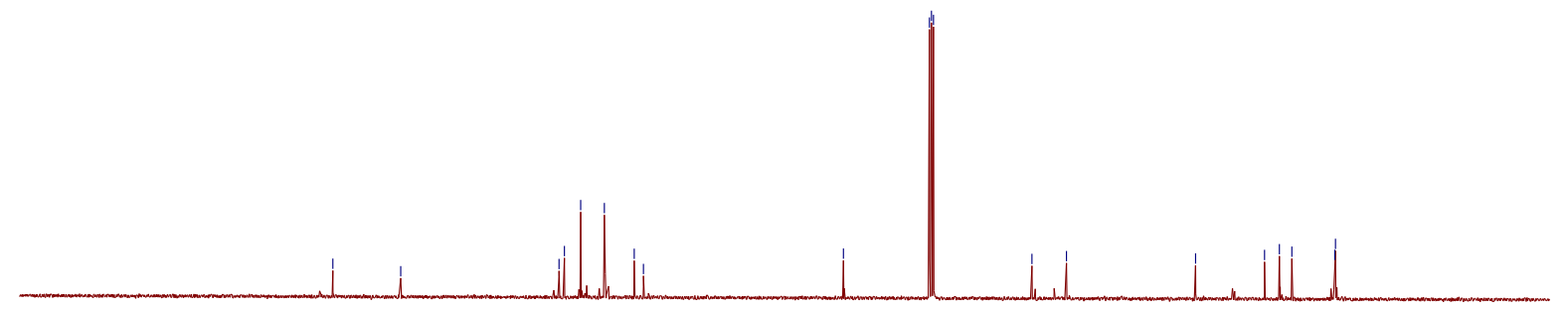

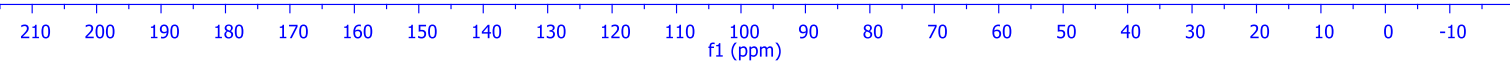

Veeru400

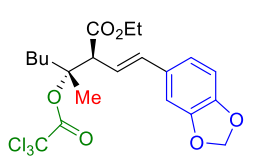

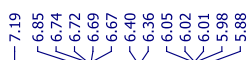

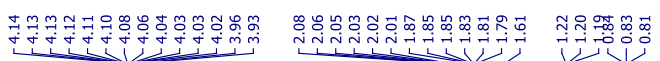

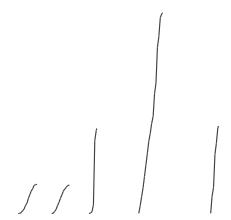

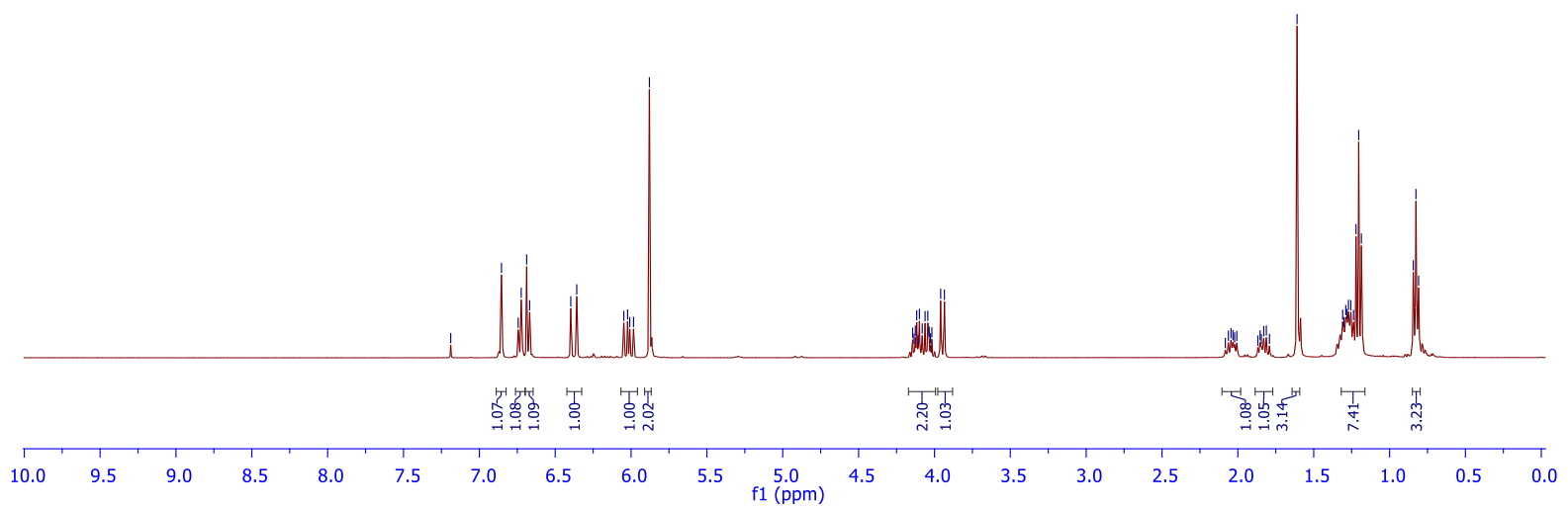


Veeru400
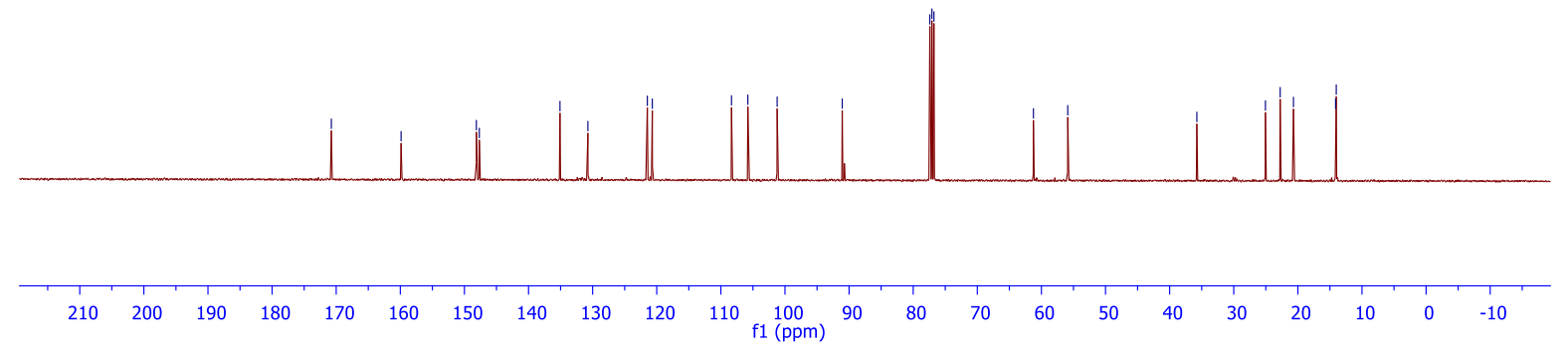

Veeru4002019

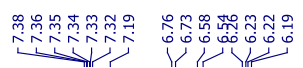

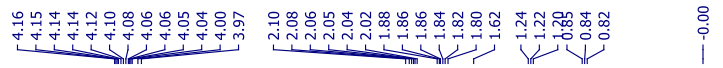
$\mathrm{Cl}_{\mathrm{O}}^{\mathrm{C}}$

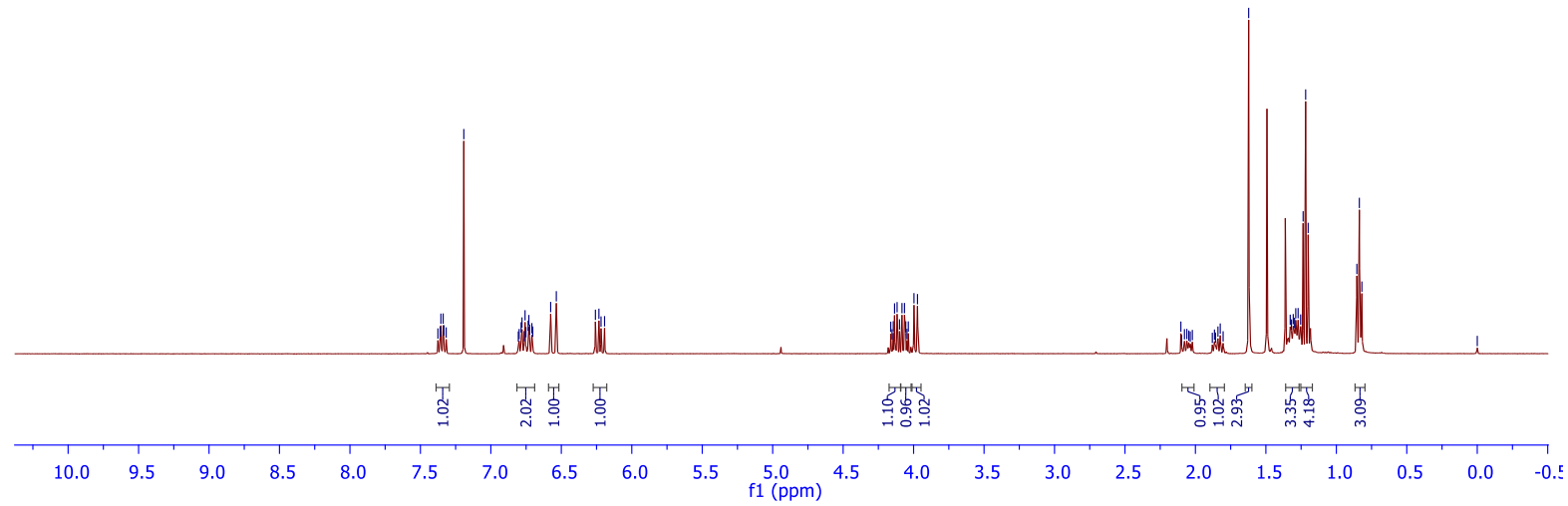



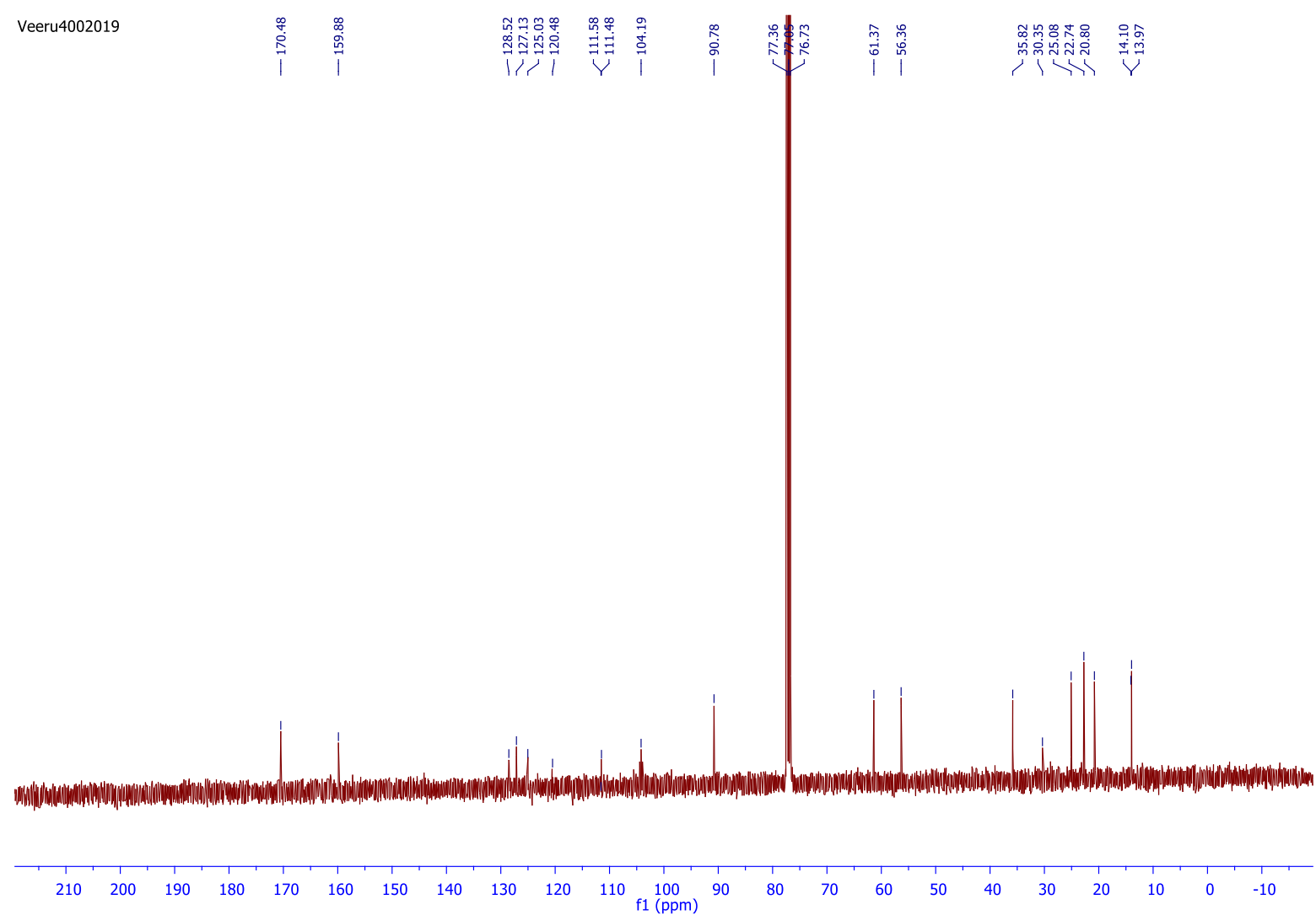

Veeru4002020

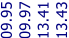

Y

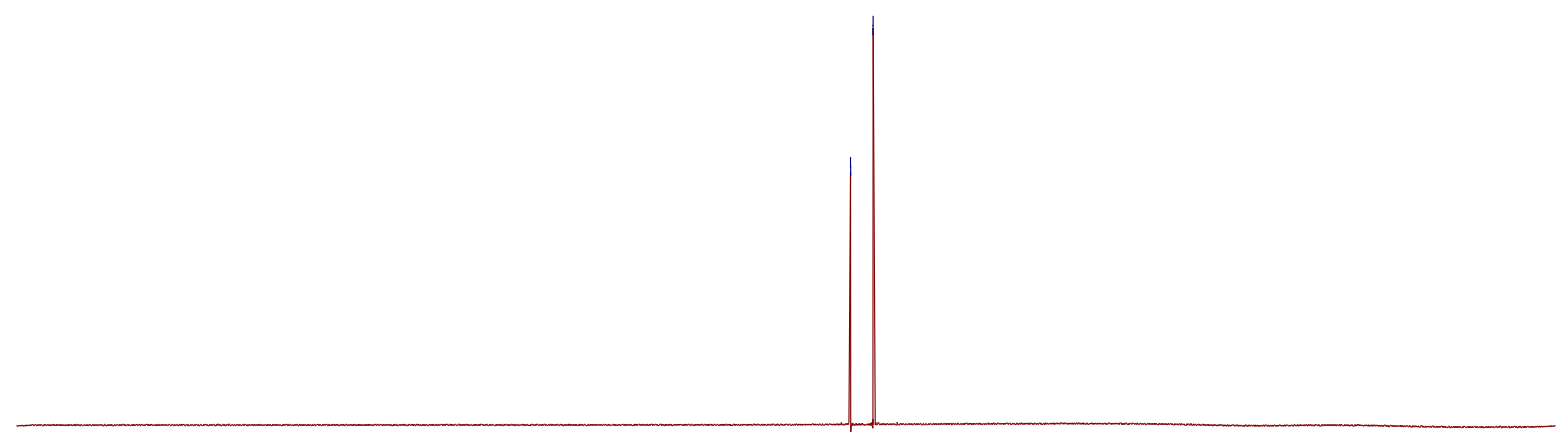

$\begin{array}{llllllllllllllllllllllll}10 & 0 & -10 & -20 & -30 & -40 & -50 & -60 & -70 & -80 & -90 & -100 & -110 & -120 & -130 & -140 & -150 & -160 & -170 & -180 & -190 & -200 & -210\end{array}$ 


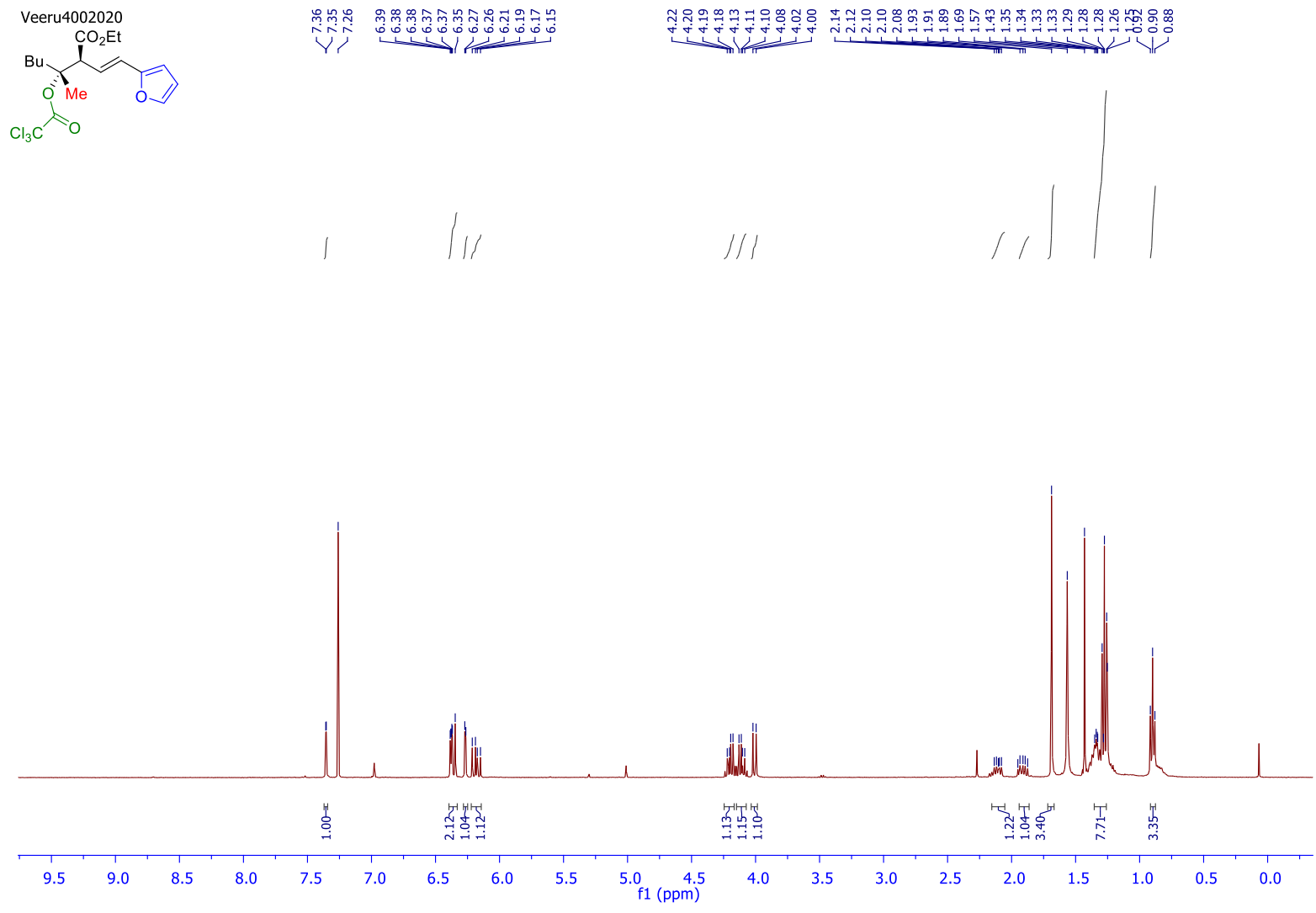

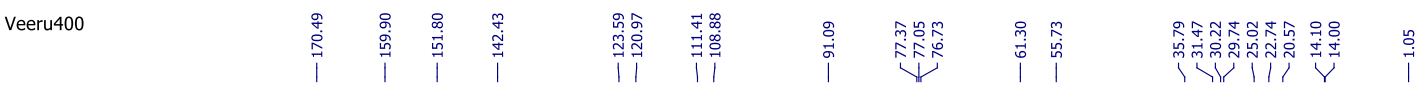

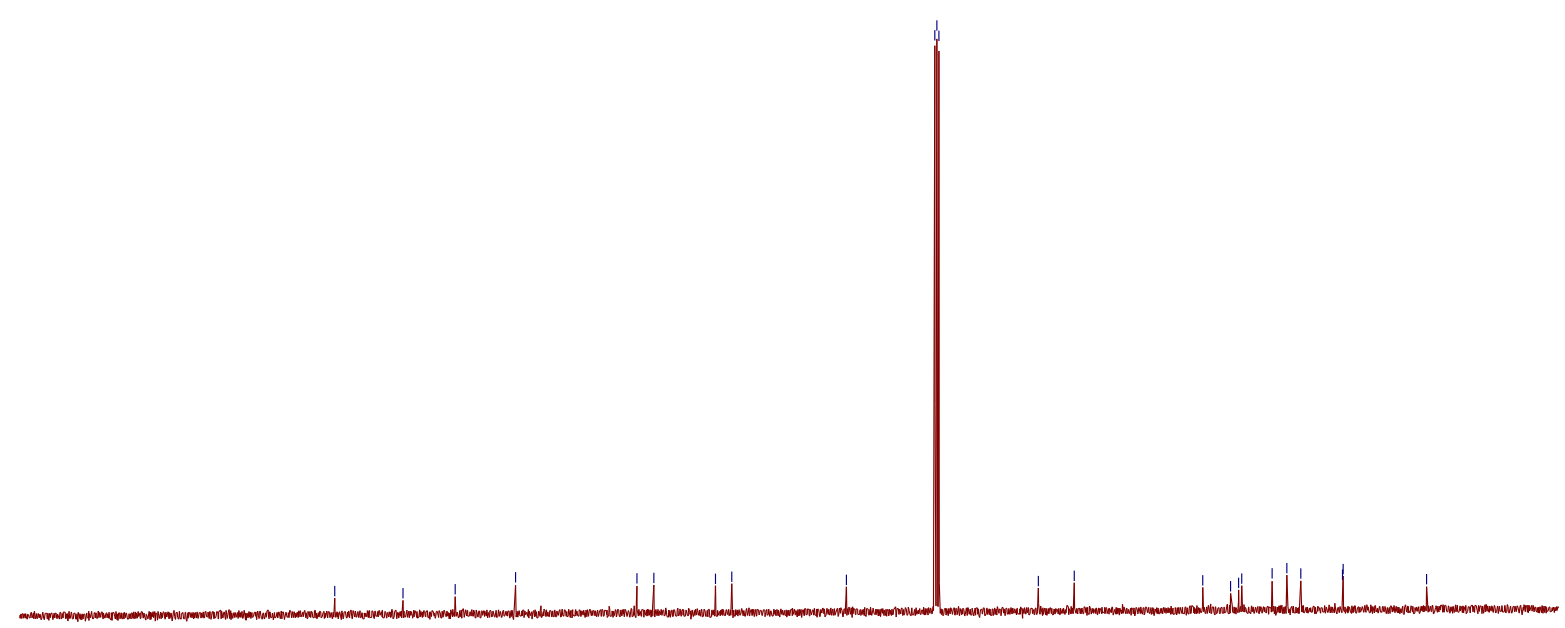

$\begin{array}{lllllllllllllllllllllllllllll}210 & 200 & 190 & 180 & 170 & 160 & 150 & 140 & 130 & 120 & 110 & 100 & 90 & 80 & 70 & 60 & 50 & 40 & 30 & 20 & 10 & 0 & -10\end{array}$ 

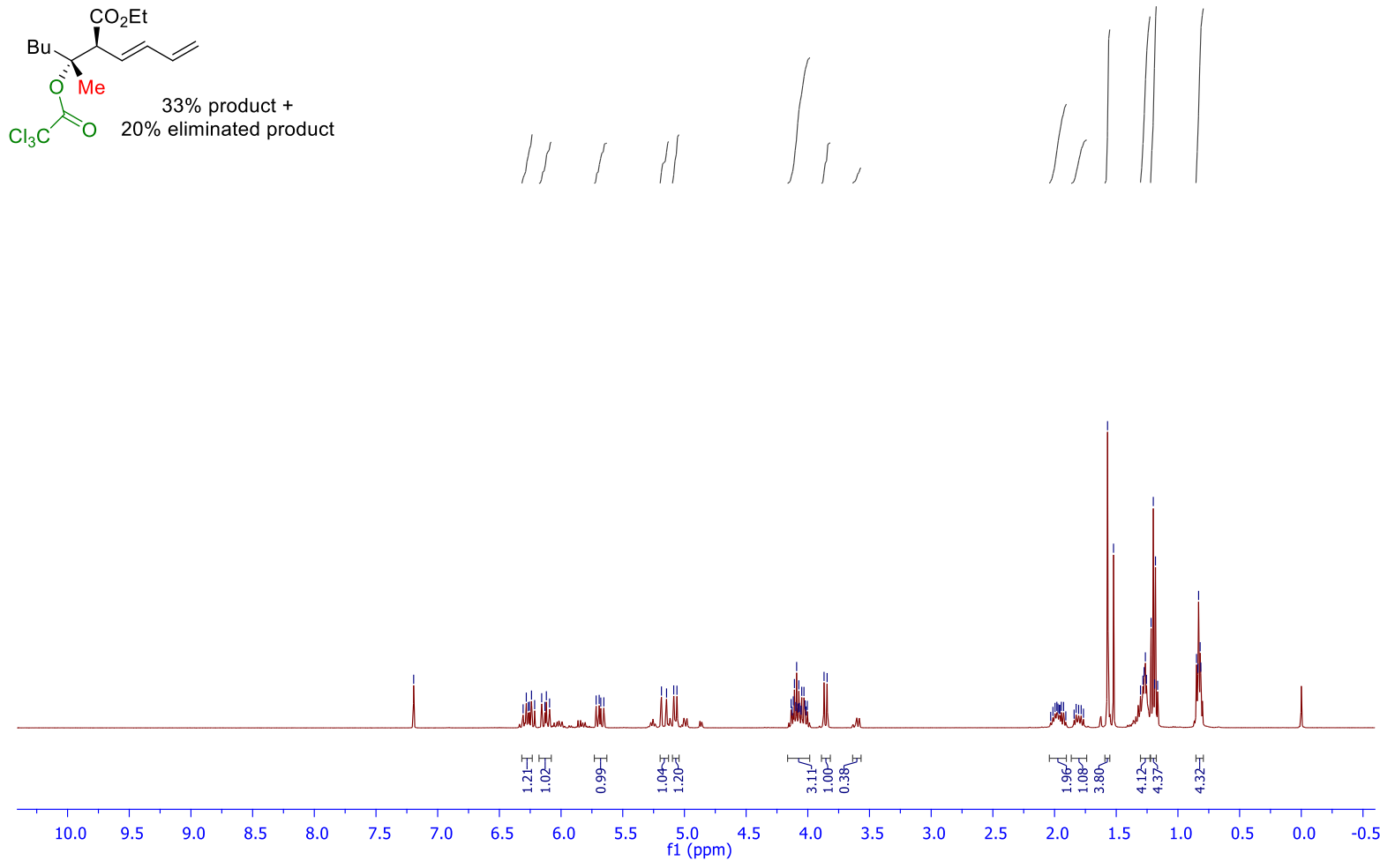

Veeru400

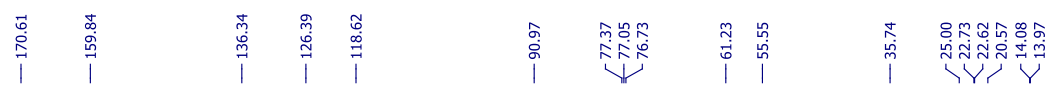

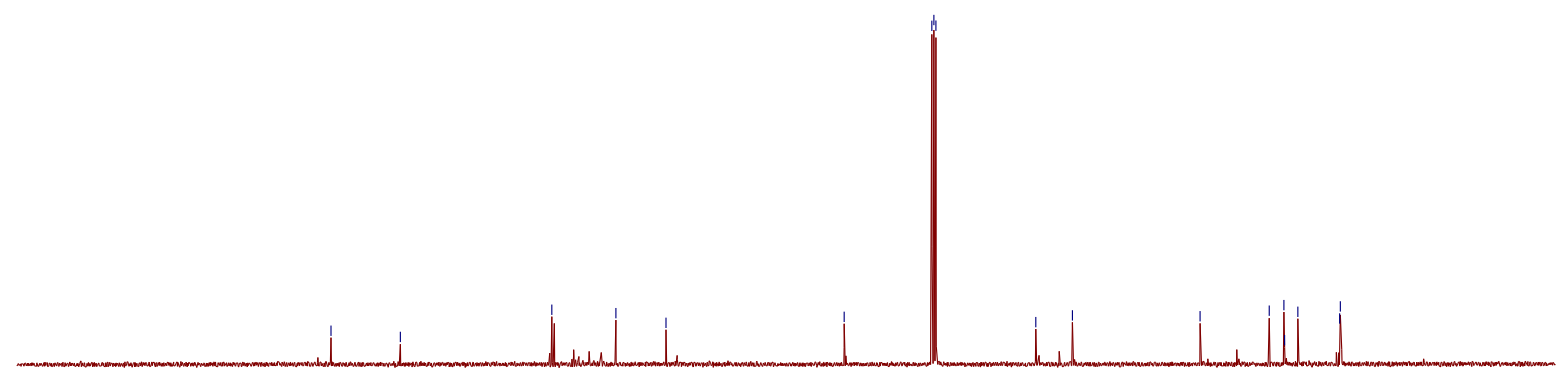

$\begin{array}{lllllllllllllllllllllllllll}210 & 200 & 190 & 180 & 170 & 160 & 150 & 140 & 130 & 120 & 110 & 100 & 100 & 80 & 70 & 60 & 50 & 40 & 30 & 20 & 10 & 0 & -10\end{array}$ 


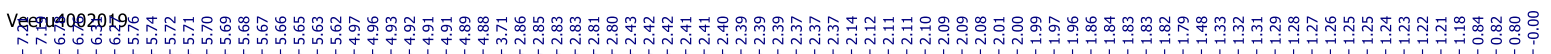
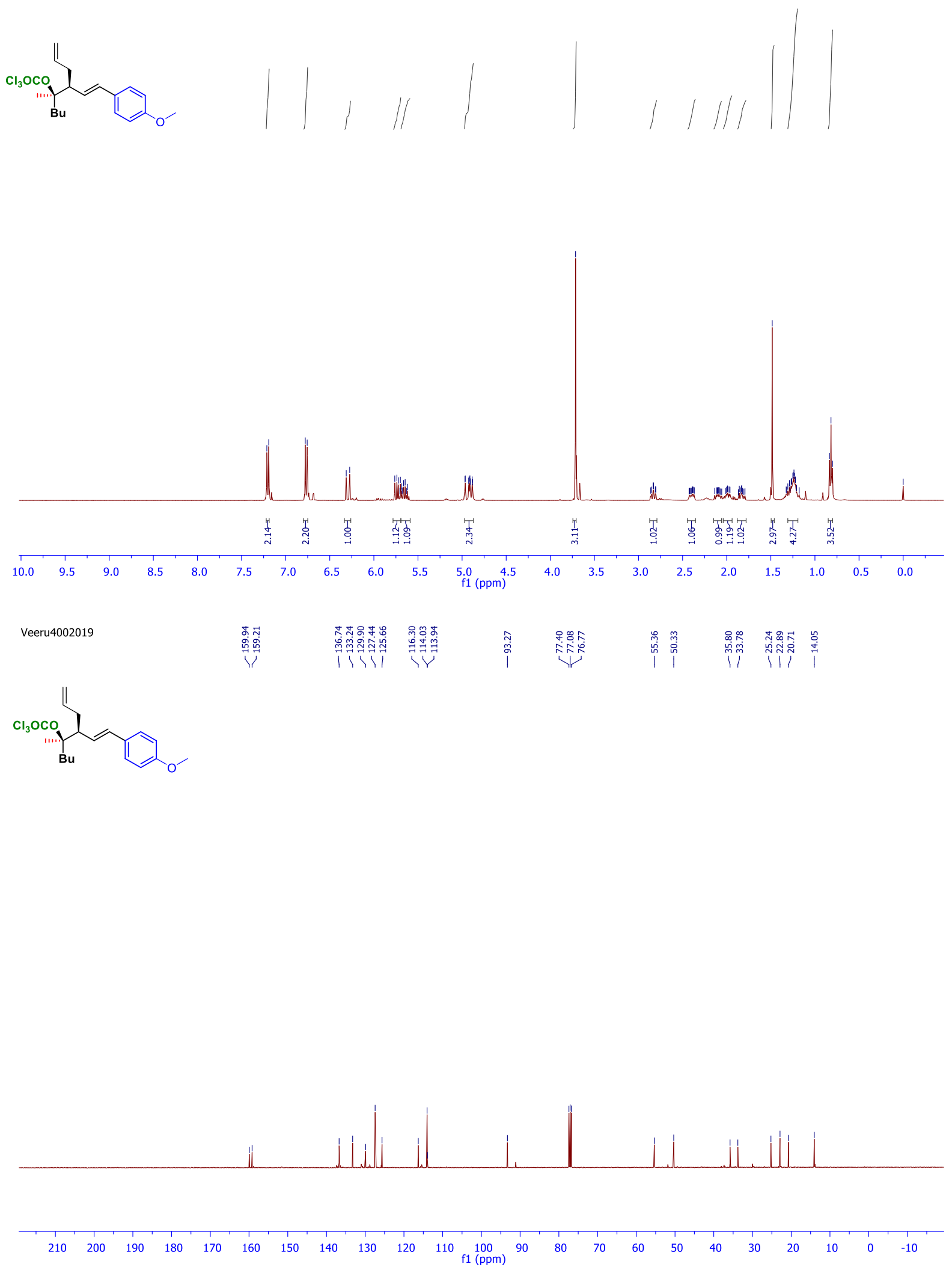

SI-76 


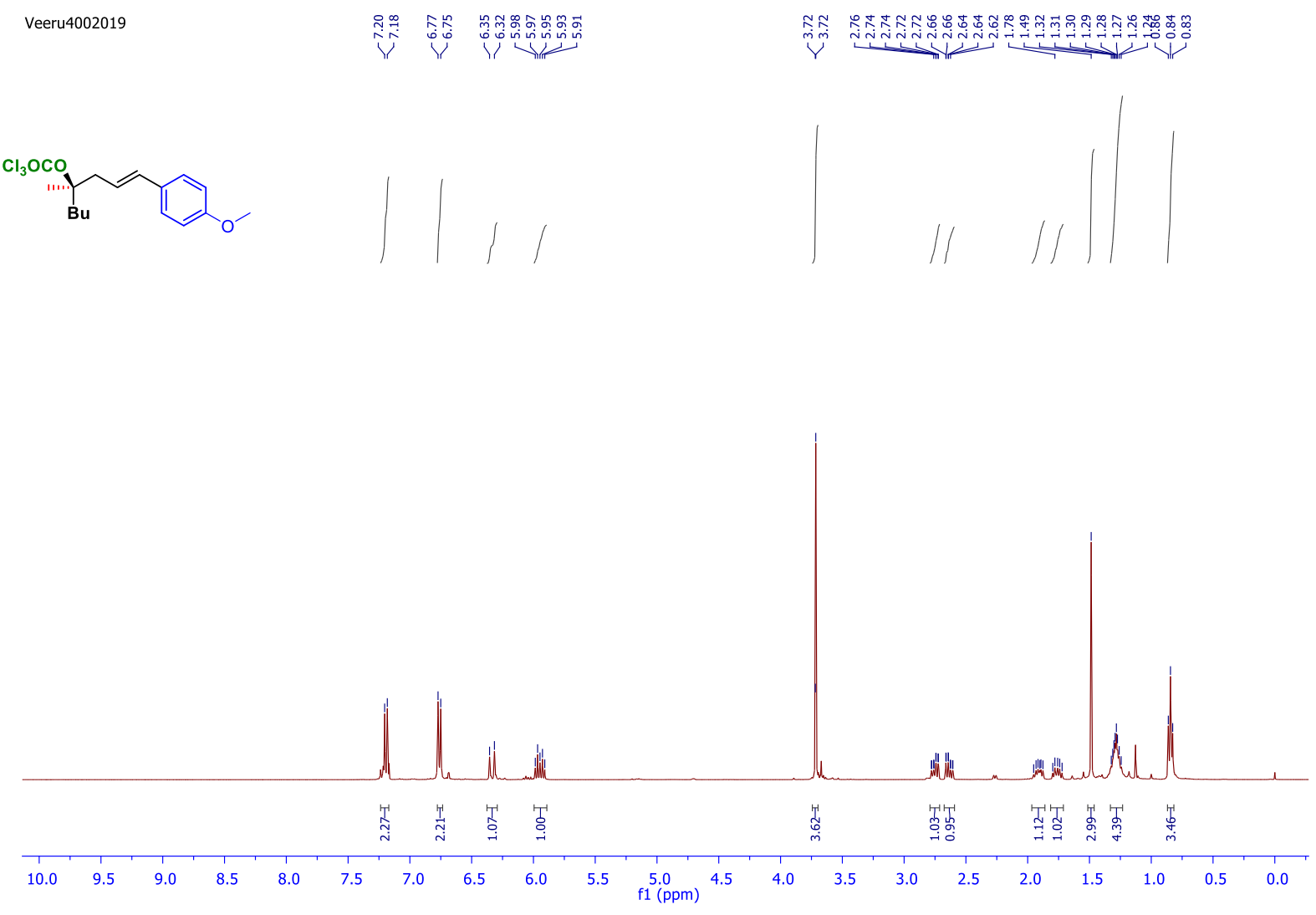

Veeru4002019

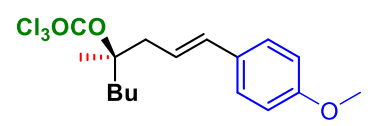

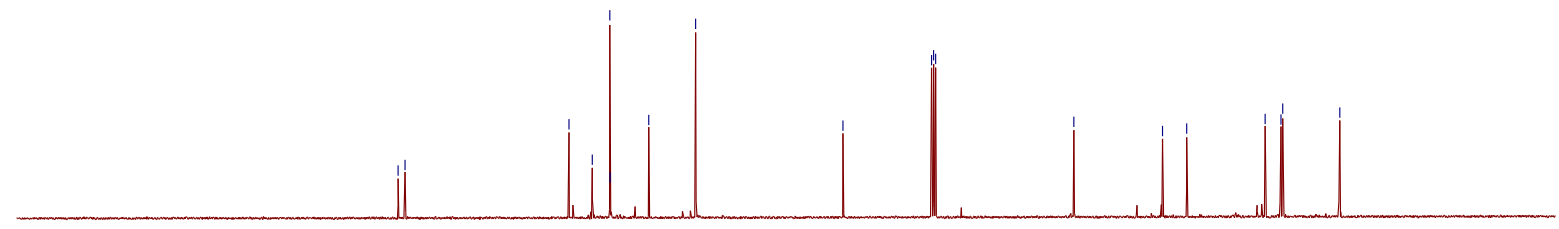

$\begin{array}{llllllllllllllllllllllllllll}210 & 200 & 190 & 180 & 170 & 160 & 150 & 140 & 130 & 120 & 110 & 100 & 90 & 80 & 70 & 60 & 50 & 40 & 30 & 20 & 10 & 0 & -10\end{array}$ 
Veeru4002019

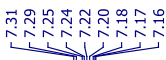<smiles>C#CC#C</smiles>

(75\% of product

of $\mathrm{Me} \quad 13 \%$ of eliminated byproduct

$\mathrm{Cl}_{3} \mathrm{C} \curvearrowright \mathrm{O}$

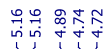

$Y \mid Y$

11

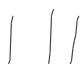

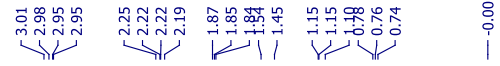

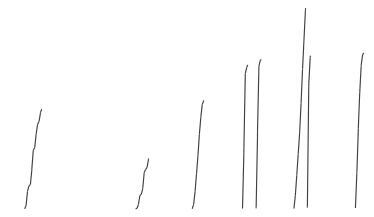

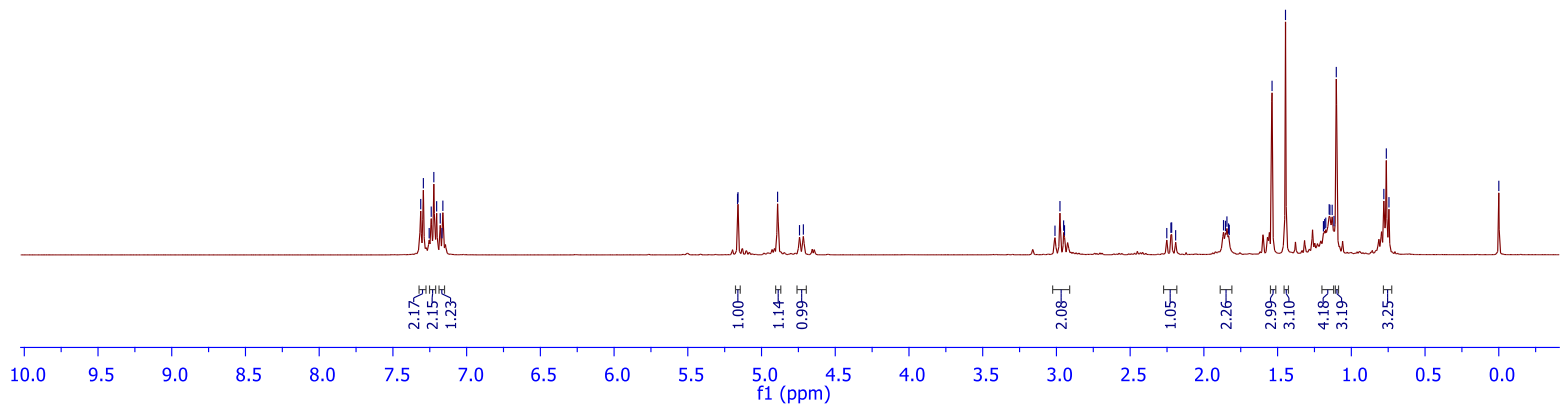

Veeru4002019

(-)

$\mathrm{Cl}_{3} \mathrm{C}$ 视

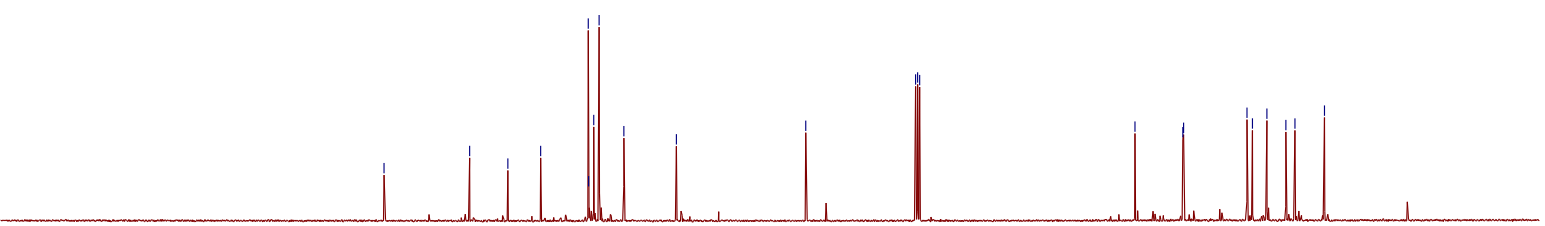

$\begin{array}{lllllllllllllllllllllllll}210 & 200 & 190 & 180 & 170 & 160 & 150 & 140 & 130 & 120 & 110 & \begin{array}{c}100 \\ \mathrm{f} 1(\mathrm{ppm})\end{array} & 90 & 80 & 70 & 60 & 50 & 40 & 30 & 20 & 10 & 0 & -10 & -20\end{array}$ 
<smiles>COc1ccc(/C=C/[C@@H](C)[C@H](Br)NC(=O)C(Cl)Cl)cc1</smiles>
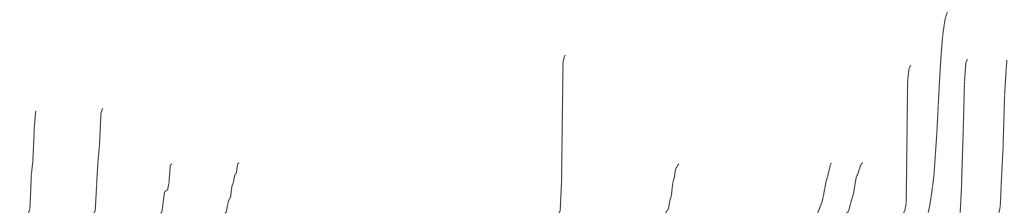

$\mathrm{Cl}_{3} \mathrm{C}$

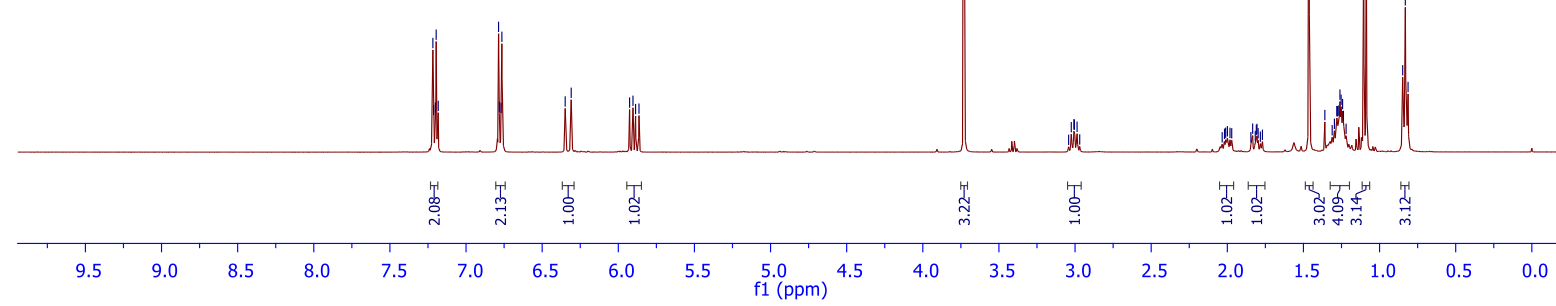

Veeru4002020

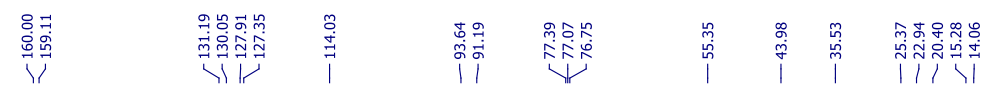

Cla

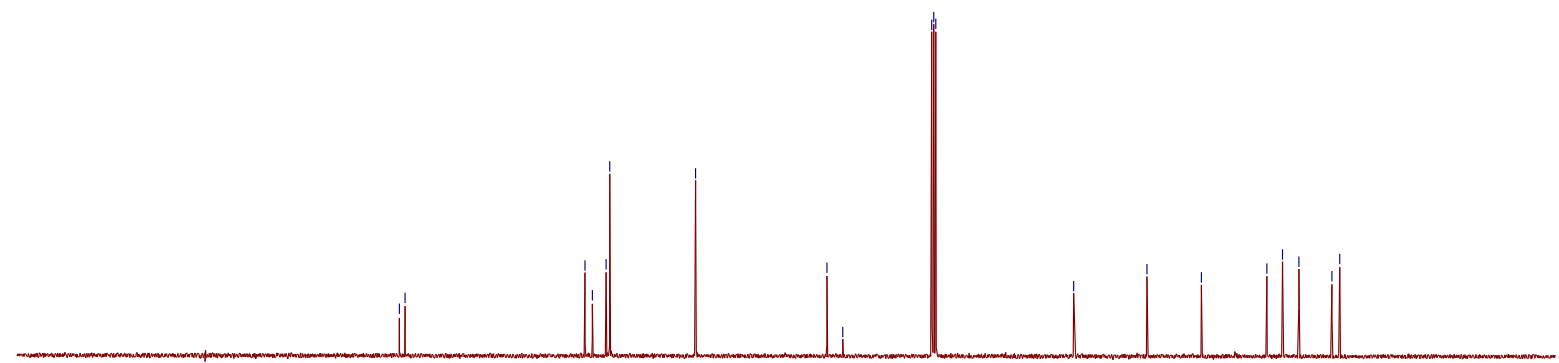

$\begin{array}{lllllllllllllllllllllllllll}210 & 200 & 190 & 180 & 170 & 160 & 150 & 140 & 130 & 120 & 110 & 100 & 100 & 80 & 70 & 60 & 50 & 40 & 30 & 20 & 10 & 0 & -10\end{array}$ 
Veeru4002019

(NO

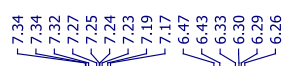

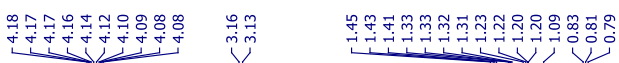

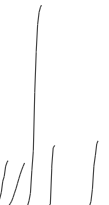

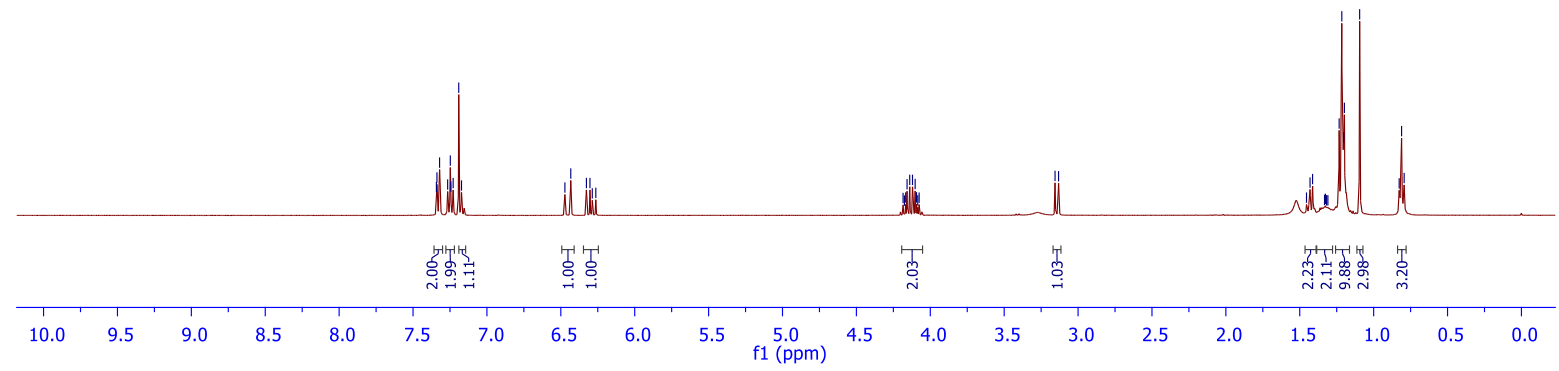

Veeru4002019

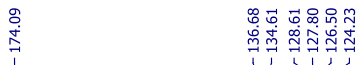

H.

诲 


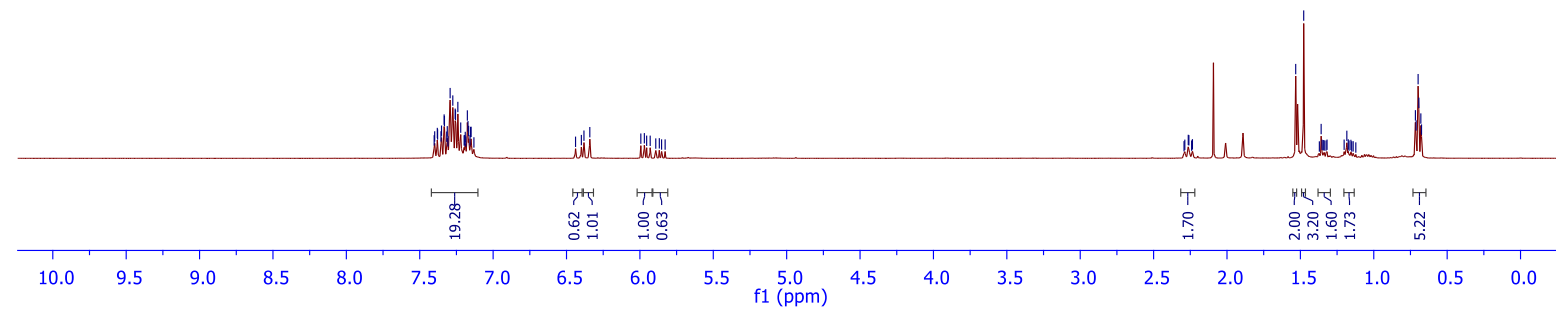

Veeru400

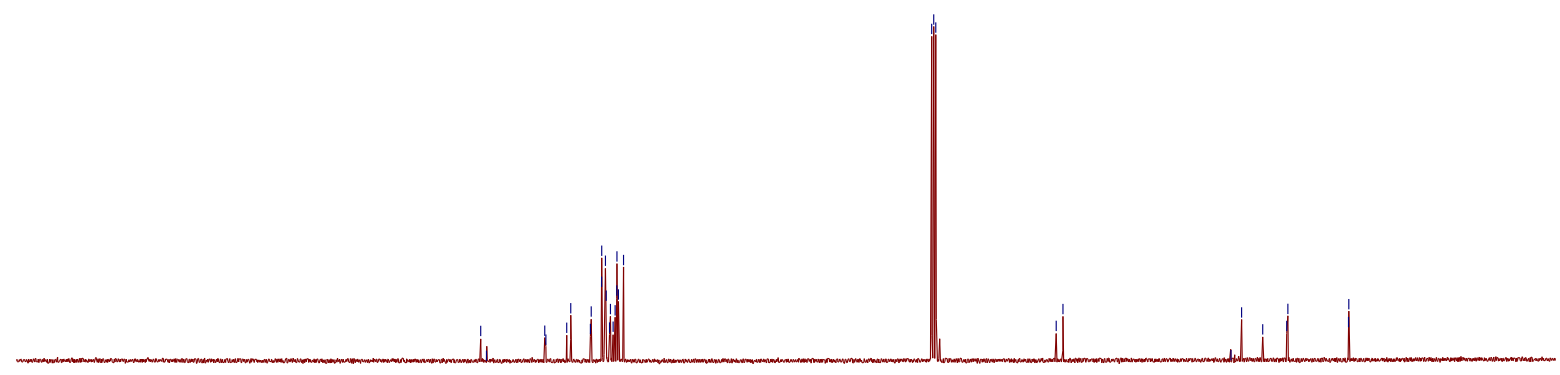

$\begin{array}{llllllllllllllllllllllllllll}210 & 200 & 190 & 180 & 170 & 160 & 150 & 140 & 130 & 120 & 110 & 100 & 100 & 80 & 70 & 60 & 50 & 40 & 30 & 20 & 10 & 0 & -10\end{array}$ 


\section{References:}

${ }^{1}$ Didier, D.; Delaye, P.-O.; Simaan, M.; Island, B.; Eppe, G.; Eijsberg, H.; Kleiner, A.; Knochel, P.; Marek, I. Modulable and Highly Diastereoselective Carbometalation of Cyclopropenes. Chem. - Eur. J. 2014, 20, 1038- 1048. b) Fa-Guang Zhang, Guillaume Eppe and Ilan Marek., Brook Rearrangement as a Trigger for Ring-Opening strained Carbocycles Angew. Chem. Int. Ed. 2016, 55, 714-718.

${ }^{2}$ Singh, S.; Bruffaerts, J.; Vasseur, A.; Marek, I. A unique Pd-catalysed Heck arylation as a remote trigger for cyclopropane selective ring-opening. Nat. Commun. 2017, 8, 14200. 